
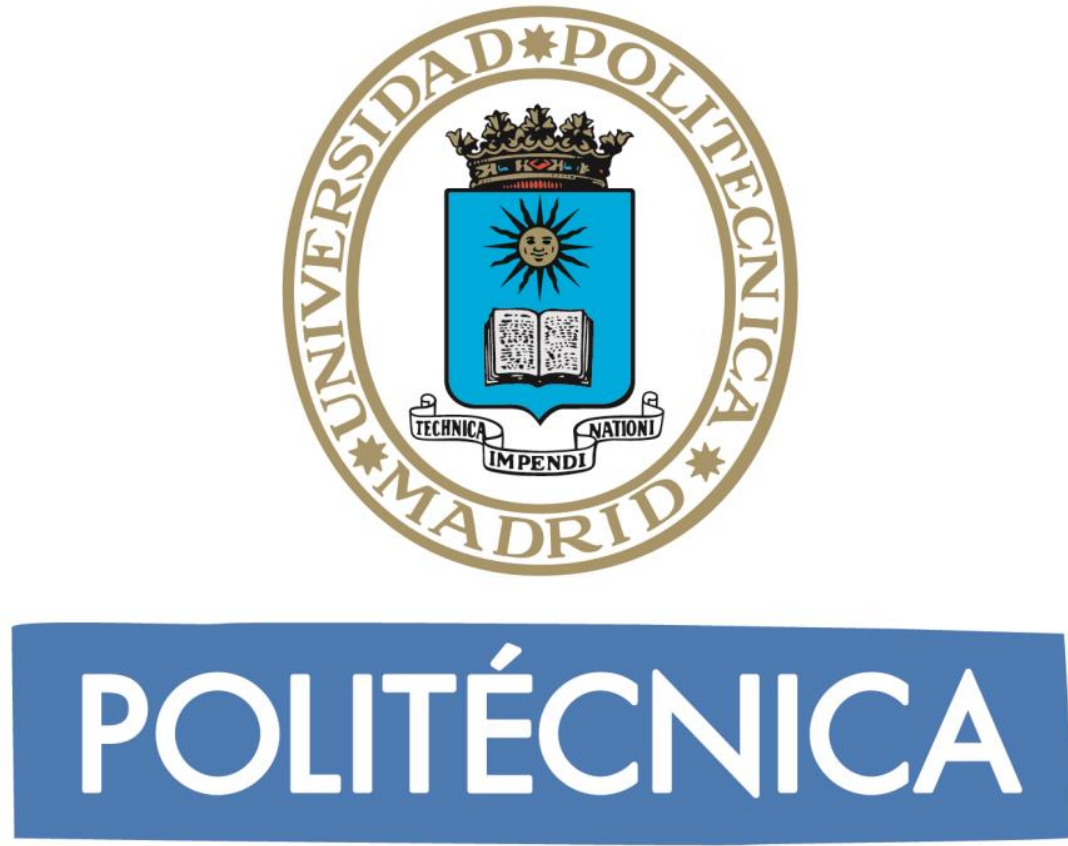

ESCUELA E.T.S DE INGENIEROS INFORMÁTICOS

\title{
Attentiveness and Engagement in Learning Activities
}

Doctoral Thesis

Doctor Dalila Alves Durães

2018 



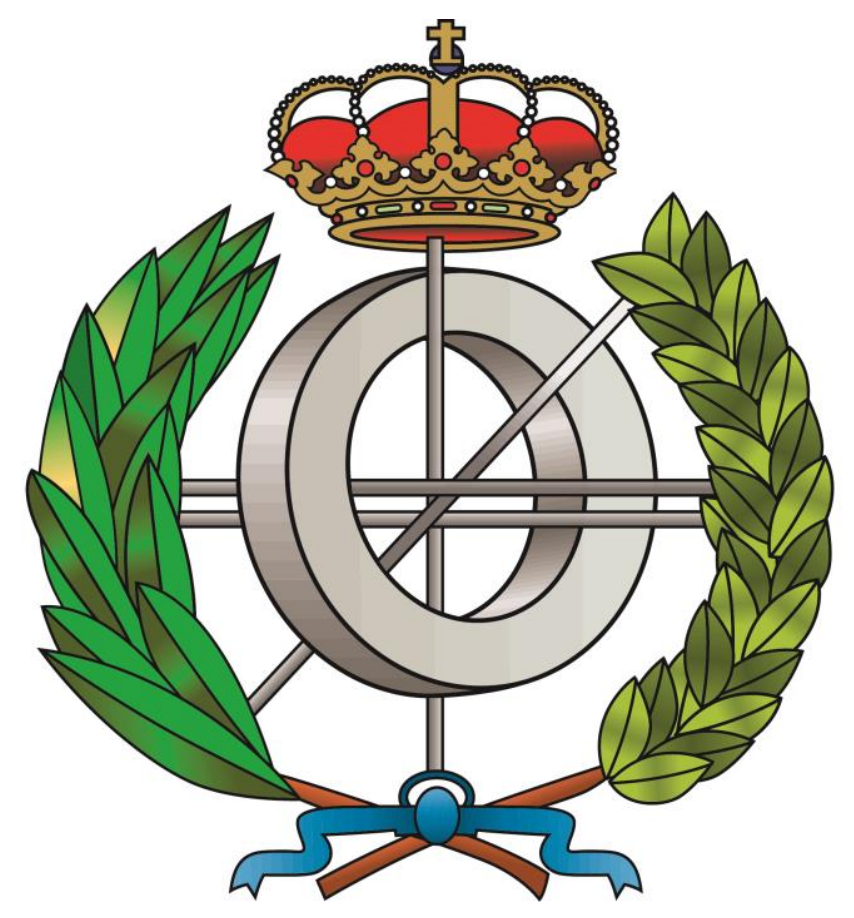

Departamento de Inteligencia Artificial E.T.S DE INGENIEROS INFORMÁTICOS

\section{Attentiveness and Engagement in Learning Activities}

Autor: Doctor Dalila Alves Durães

Director: Doctor Javier Bajo Perez

Director: Doctor Paulo Jorge Freitas de Oliveira Novais 

Original in German:

"Daher ist die Aufgabe nicht sowohl zu sehen was noch keiner gesehen hat, als bei Dem was Jeder sieht, zu denken was noch Keiner gedacht hat. "

\section{English:}

"So the task is, not so much to see what no one has seen yet, but to think what nobody has yet thought, about what everybody sees." 



\section{Ag}

\section{ACKNOWLEDGMENTS}

Elaborating this thesis and developing this doctoral project would not have been possible without the contribution of those to whom I refer below. To all of them my sincere thanks:

To my family, especially my husband and son Antero. As much as I want to find words sufficiently demonstrative of my recognition... I cannot!! You were fantastic. Thank you for your support, for your presence, strength, patience, motivation and love.

Thank you to all my colleagues, but especially to those who were close to me. Thank you for all the words of support, comfort and encouragement that have been very useful to me in certain moments. Special thank you for the patience you have given me without ever denying your help.

Special thanks to my colleagues of Synthetic Intelligence Group (ISLab): Cesar Analide, Filipe Gonçalves, Fábio Silva, Marco Gomes, João Ramos, Tiago Oliveira, António Silva, Ricardo Martins, Ramon Toala, Bruno Fernandes, Leandro Freitas, and Jaime Rincon. I am very happy to have met you and to have collaborated with you and who helped me during this project.

Likewise, special thanks to CECII (Center for Innovation and Research in Business Sciences and Information Systems) colleagues at Polytechnic of Porto 
Institute, especially to Davide Carneiro for the support he gave me at my stay and to Ricardo Santos for trusting my work. My sincere thanks.

I am grateful to the english colleagues of the High School of Caldas of Taipas, especially to Ana Guedes, Carla Abreu, Paulo Botelho and Etelvina Silva who, in the beginning, corrected the English of my papers. A special thanks to Rosa Barros that review my entire thesis.

Thank you to the University of Minho for giving me the opportunity to develop part of my research in the Synthetic Intelligence group (ISLab) of Algoritmi Center.

To Professor Paulo Novais who has opened a new world for me and for that, I will be forever indebted to him. Professor Paulo Novais always provided the guidance I needed and gave me the opportunity to work with him at the Intelligent Systems Laboratory, which was an immense pleasure and a fantastic experience. His experience and advice have helped me to pursue my goals and I cannot express how grateful I am.

I thank the professors of my Ph.D. from the Technical University of Madrid who have endeavored to convey their knowledge and in this way have contributed to increase my knowledge with a good disposition, commitment and joy.

A special thanks to my supervisor, Professor Javier Bajo, for the great sympathy, patience, suggestions, and the availability demonstrated during these three years, allowing me to get here. It was a privilege to work with you. 


\section{$A b$}

\section{ABSTRACT}

The Human being is currently under increased demand for attention, a result of a society that is moving faster. In most of the so-called developed countries, workers have nowadays increasingly busier activities. This makes them stretch their limits to find time for children, sports activities, and other. This necessary extra time is frequently obtained at the expense of shorter periods of sleep or rest. Although this effect may not be readily visible, they may have consequences at many other levels than health, including emotions, results, attention and social behavior, among others.

For these reasons, school and systems of e-learning must create environments that can involve students and capture their attention and engagement. Advances in computers and wireless technologies have also had an impact on the educational system, thus generating a new approach for Ambient Intelligent Systems (Aml systems). The rapid development of these technologies combined with the access to content in a wide variety of settings, allows learners to experience new learning situations beyond the school's walls.

The need for qualified people is growing exponentially, requiring limited resources allocated to education/training to be used most efficiently. Learning and elearning systems can allow some flexibility for students who have complicated schedules and obligations. However, some problems can occur: (1) relying on learning theories, it is crucial to improve the learning process and mitigate the issues 
that may arise from technologically enhanced learning environments; (2) each student presents a particular way of assimilating knowledge, i.e. his/her learning style. It is essential that these systems adapt to the learning preferences of the students.

This work deals with the issue of attention monitoring as a form of engagement, with the aim of providing a non-intrusive, reliable and easy tool that can be used freely in schools or organizations, without changing or interfering with the established working routines. We propose an intelligent learning system able to monitor the patterns of students' behavior during lessons, to support the teaching procedure within school environments. The system used behavior patterns based on mouse dynamics, keystroke dynamics, student's attention, and lessons activity. The idea highlights the main biometric behavioral variations for each activity and bases the set of attributes relevant to the development of machine learning classifiers for the prediction of students' learning preference. The objective is to show that there are still mechanisms that can be explored to understand better the complex relationship between human behavior, attention, and learning, which could be used for the implementation of better learning strategies. After improving learning systems in a learning and e-learning environment it is possible to predict students' behavior in an (e)learning lesson, based on their interaction with technological devices. 


\section{$\operatorname{Re}$}

\section{RESUMEN}

Actualmente, el ser humano demanda una mayor atención individualizada, como resultado de una sociedad que evoluciona rápidamente. En la mayoría de los denominados países desarrollados, los trabajadores tienen actividades que cada vez los mantienen más ocupados. Esto hace que dichos trabajadores tengan que ir más allá de sus límites con el fin de encontrar tiempo para sus hijos, deporte y otras actividades. Este tiempo extra se obtiene, frecuentemente, sacrificando períodos de sueño y descanso. Aunque los efectos en muchas ocasiones pueden no ser fácilmente visibles, tienen consecuencias en aspectos más allá de la propia salud, tales como emocionales, de concentración y sociales, entre otros.

Por ello, los centros de formación, así como los propios sistemas de elearning deben ser capaces de buscar nuevas soluciones que se adapten a la nueva realidad, permitan captar la atención de los estudiantes, conseguir una mayor inmersión en el proceso formativo, así como incrementar su compromiso personal y preparación para afrontar los retos de la nueva sociedad. Los avances proporcionados por la aparición de los ordenadores y de las tecnologías inalámbricas han tenido un gran impacto en el sistema educativo, generando así, un nuevo enfoque en lo referente a Sistemas de Inteligencia Ambiental o Entornos Inteligentes (sistemas Aml). Por otro lado, la demanda de personal cualificado está creciendo exponencialmente requiriendo, eso sí, de recursos limitados en educación y formación que deben ser utilizados de la manera más eficiente. 
El rápido desarrollo de estas tecnologías combinado con el acceso a los contenidos educativos mediante una amplia variedad de opciones, permite a los estudiantes acceder a nuevas experiencias de aprendizaje que van más allá de los muros de la escuela. Una de las ventajas de los sistemas de aprendizaje y elearning es que pueden ofrecer cierta flexibilidad a los estudiantes que están sujetos a horarios y obligaciones. Sin embargo, pueden surgir algunos problemas: (1) Basándose en las teorías de aprendizaje, es crucial mejorar el proceso de aprendizaje y mitigar los problemas que puedan surgir en los entornos de aprendizaje que utilizan las tecnologías en el aula. (2) Cada estudiante presenta una forma particular de asimilar el conocimiento, dependiendo de su modelo personal de aprendizaje. Por tanto, es esencial que estos sistemas se adapten a las preferencias y necesidades de aprendizaje de cada estudiante.

Este trabajo aborda el problema de la monitorización de la atención de los estudiantes en entornos de aprendizaje, con el objetivo de obtener una herramienta no intrusiva, confiable y de implantación sostenible en escuelas u organizaciones, sin cambiar o interferir con las rutinas de trabajo establecidas. Se propone un sistema de aprendizaje inteligente, capaz de controlar los patrones del comportamiento de los estudiantes durante las clases, para apoyar el procedimiento de enseñanza dentro de los entornos escolares. El sistema que se presenta utiliza patrones de comportamiento basados en la dinámica del ratón, la dinámica de la pulsación sobre teclado, así como la atención del alumno y la actividad durante las lecciones. La idea principal es identificar las principales variaciones biométricas de comportamiento para cada actividad tenida en cuenta, así como el conjunto de atributos más relevantes a la hora de desarrollar clasificadores basados en aprendizaje automático como método de predicción de las preferencias de aprendizaje de los estudiantes. El objetivo es mostrar que todavía existen mecanismos que se pueden explorar para comprender mejor la compleja relación existente entre el comportamiento humano, la atención y el aprendizaje, y que podrían utilizarse para la implementación de mejores estrategias de enseñanzaaprendizaje. De esta forma, en este trabajo se pretende demostrar que la mejora de los sistemas de aprendizaje en un entorno e-learning, permite predecir el comportamiento de los estudiantes en una clase basada en la interacción con dispositivos tecnológicos. 


\section{$K w$}

\section{KEYWORDS}

1. Affective Computing (AC) - According to (Hassin, Aziz, \& Norwawi, 2004), the appearance of $A C$ is related to the needs to put computers interacting directly, thinking, receiving, and transmitting people's personalities.

2. Affective Learning - According to the literature, there is a connection between emotion and the learning process (McDaniel, et al., 2007) (Regan, 2003). Although this process is not simple or direct. It is accepted that positive and negative emotional states can cause different kinds of thinking and can have an effect on the learning perspective.

3. Attention - Attention means focusing on clear thinking, among one of several subjects or objects that may capture the mind simultaneously. Attention implies the concentration of mental powers upon an object by close or careful observing or listening, which is the ability or power to concentrate mentally.

4. Behavior Biometrics - Mouse and keyboard tracking are techniques also used to measure and classify attention. These techniques have already been used to measure other variables such as stress (Carneiro, Novais, Pêgo, Sousa, \& Neves, 2015), and mental fatigue (Pimenta, et al., 2015), (Pimenta, Carneiro, Neves, \& Novais, 2016).

5. Engagement - In learning activities engagement is predictive of academic proficiency, motivation, and task persistence, with the balance between learner 
interest and task demands determining the strength of that engagement (Hoffman \& Nadelson, 2010).

6. Intelligent Environment - A system able to monitor the patterns of students' behavior during lessons, to predict students' behavior in a lesson, based on their interaction with technological devices. The system highlights the main biometric behavioral variations for each activity and based on the set of relevant attributes, develop a machine learning classifiers for the prediction of students' learning preference and their attentiveness.

7. Learning - Learning begins a long time before school and continues for the even longer time after school. It happens quickly and in a great number of different ways and settings (Pritchard, 2009).

8. Learning Style - A learning style is a method that allows an individual to learn better. Different people learn in different ways, each one preferring a different learning style. Everyone has a mix of learning styles, but some people may find that he or she has a dominant style of learning. Others may find that they have different learning styles in different circumstances.

9. User Behavior - Each person has his/her own behavior. This behavior can be derived from several factors, such as the biological characteristics of the user, the characteristics of the task, and environmental factors. 


\section{Co}

\section{CONTENTS}

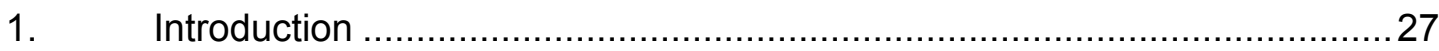

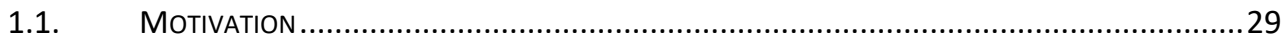

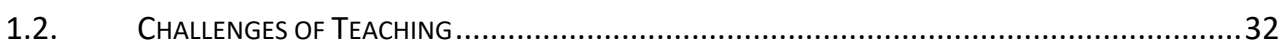

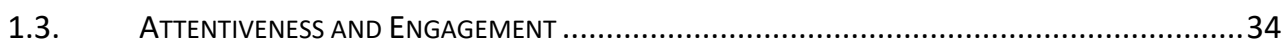

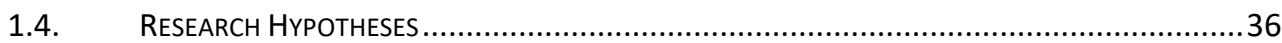

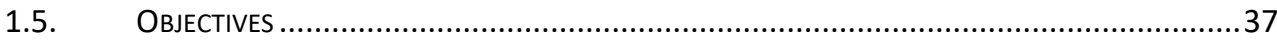

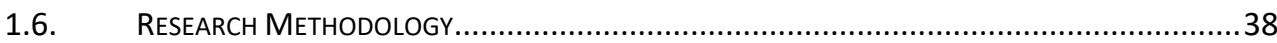

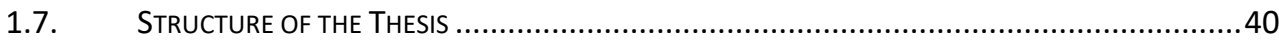

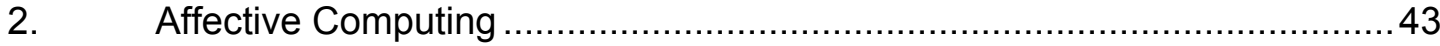

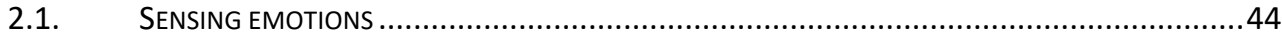

2.1.1. Discrete emotional theories ..................................................................................45

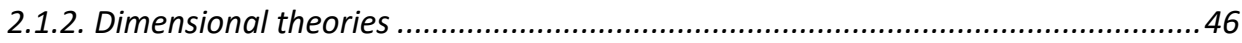

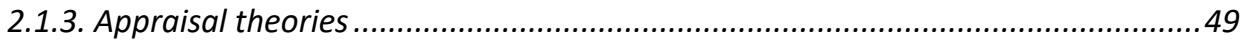

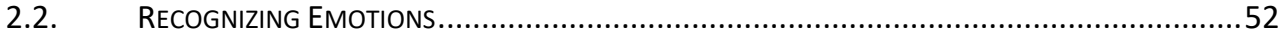

2.2.1. Facial Emotions ...........................................................................................52

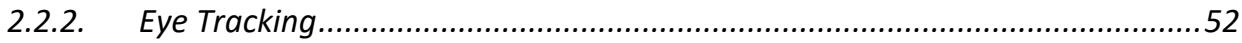

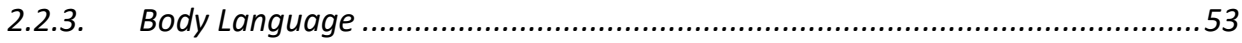

2.2.4. Emotional Speech Recognition...............................................................53

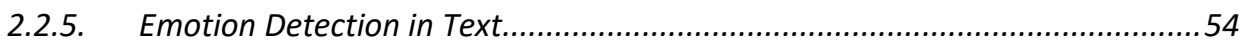

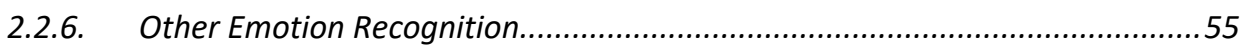

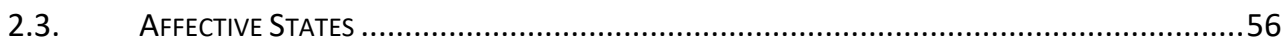




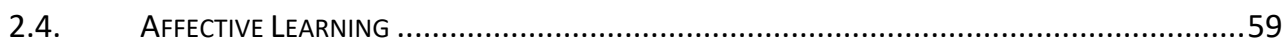

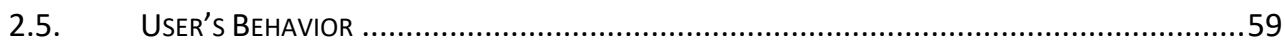

2.5.1. Pattern Interactions using Behavior Biometrics .......................................61

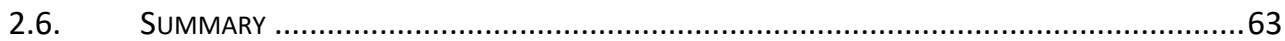

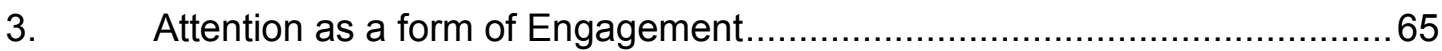

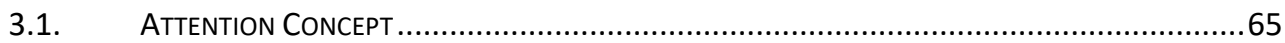

3.1.1. Attention concept in the field of philosophy ..........................................66

3.1.2. Attention concept in the field of Experimental Psychology ...........................68 68

3.1.3. Attention concept in the field of Cognitive Psychology................................69

3.1.4. Attention concept in the field of Cognitive Neuroscience .............................70

3.1.5. Attention concept in the field of Computer Science .................................... 71

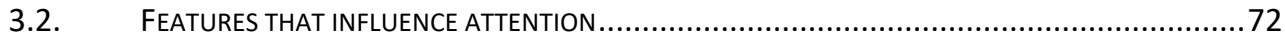

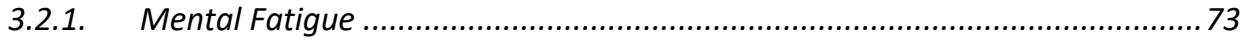

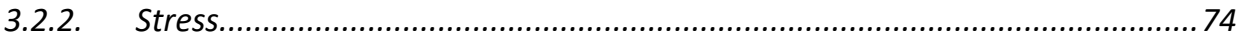

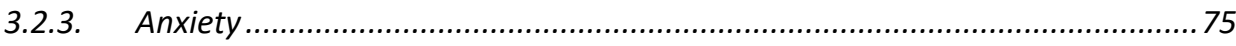

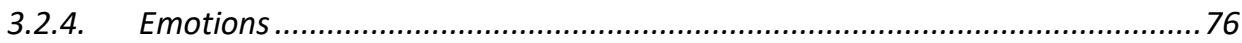

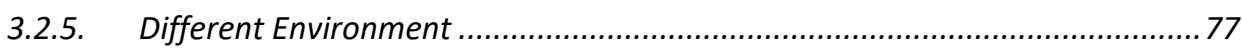

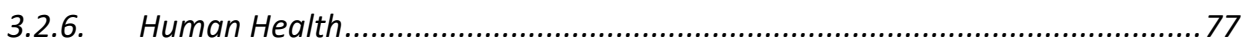

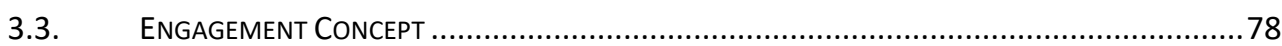

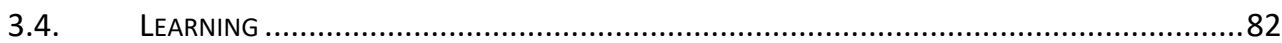

3.4.1. Behaviorism theories ....................................................................... 83

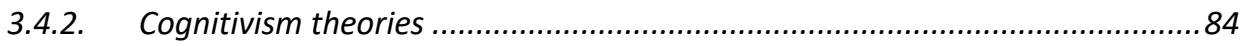

3.4.3. Constructivist theories ................................................................... 85

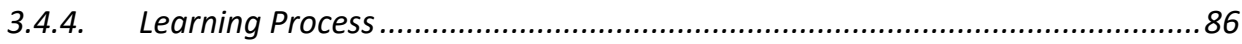

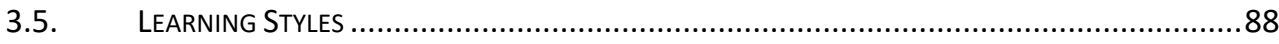

3.5.1. Kolb's Learning Style Model........................................................... 89

3.5.2. Honey and Mumford Learning Model...................................................93

3.5.3. Felder-Silverman Learning Model................................................... 95

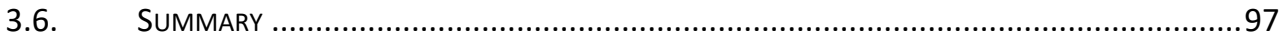

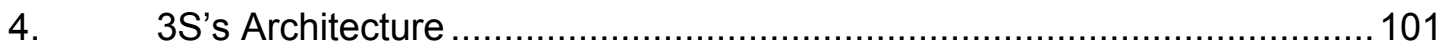

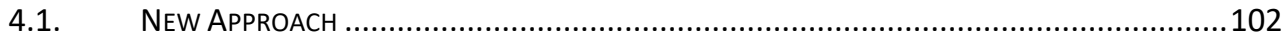

4.2. DYNAMIC FRAMEWORK OF ATTENTION ANALYSIS.................................................... 104

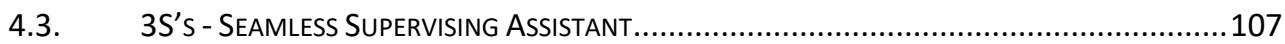

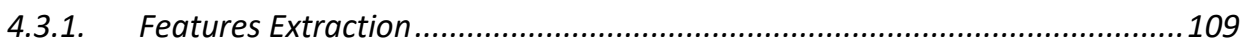

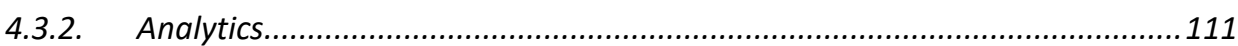


4.3.2.1. Analytics Data from Mouse and Keyboard Interaction ....................................... 111

4.3.2.2. Analytics Data from Work-related Task................................................................. 114

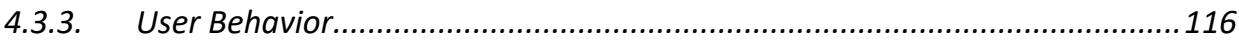

4.3.4. Emotions ...................................................................................... 117

4.3.5. Classification of Attention Level....................................................... 118

4.3.6. Learning Style's Identification.......................................................... 123

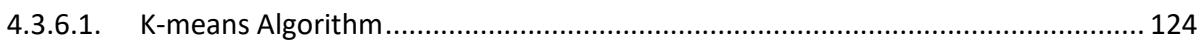

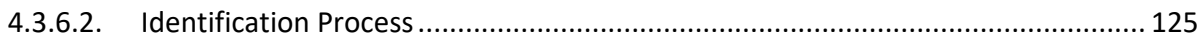

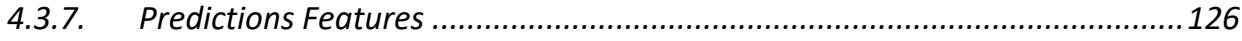

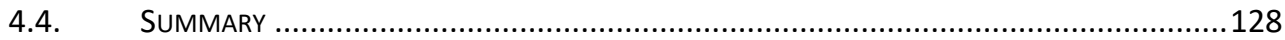

5. Analysis of Practical Results ..................................................... 129

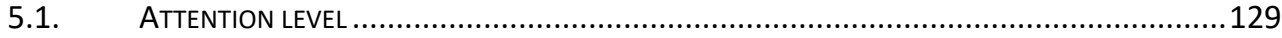

5.1.1. Case study 1 - Normal and Assessment Lesson ...................................... 130

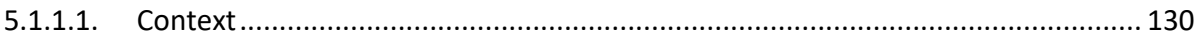

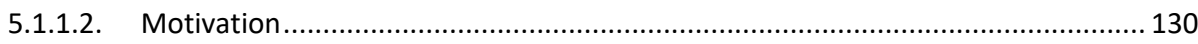

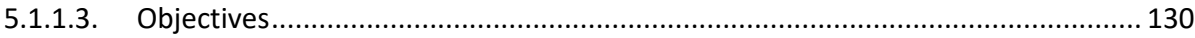

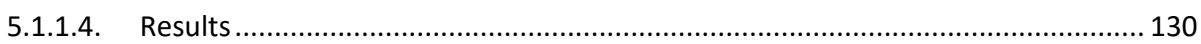

5.1.2. Case study 2 - Work-Task related.................................................. 135

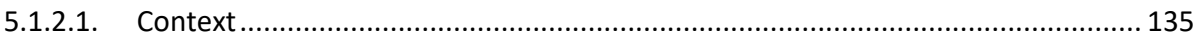

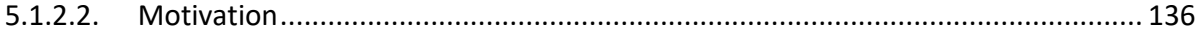

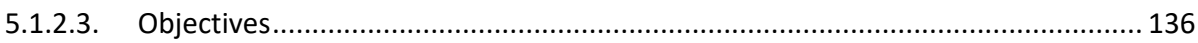

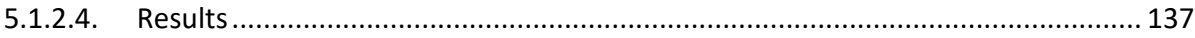

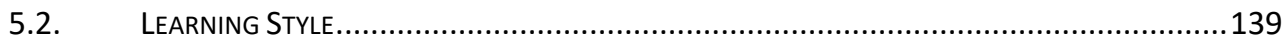

5.2.1. Case study 3 - Learning Style Influence in User Behavior..........................139

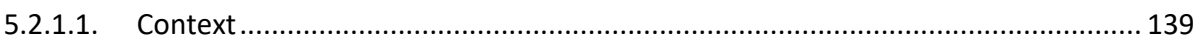

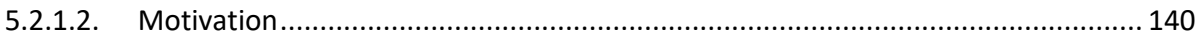

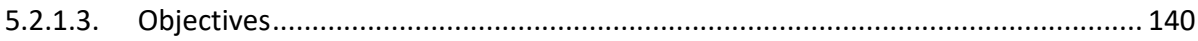

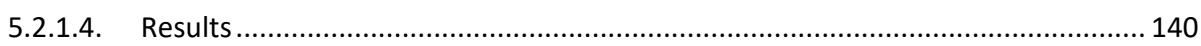

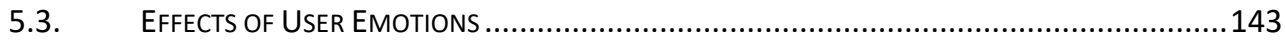

5.3.1. Case study 4 - Effects of User Emotions .............................................. 143

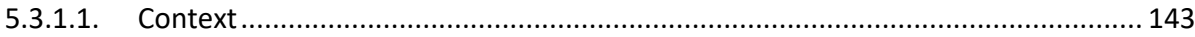

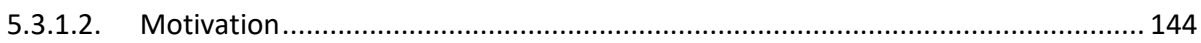

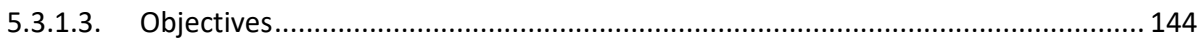

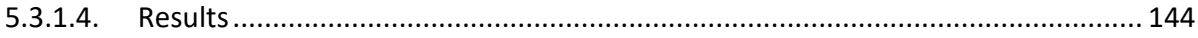

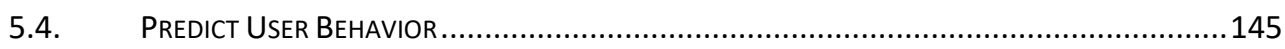

5.4.1. Case study 5-Forecasting Student's Preference in E-learning Systems ........ 145

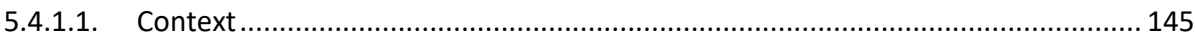




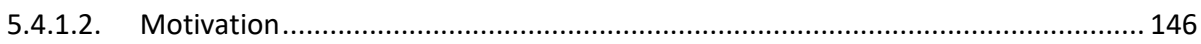

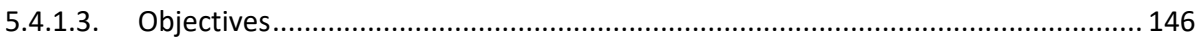

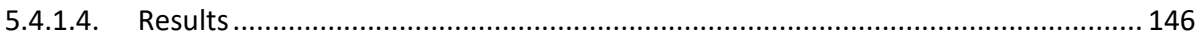

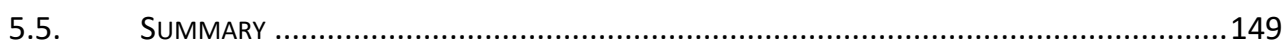

6. Publications Composing the Doctoral Thesis................................................... 151

6.1. Using Computer Peripheral Devices to Measure Attentiveness ............................ 152

6.2. SUPERVISING AND IMPROVING ATTENTIVENESS IN HUMAN COMPUTER INTERACTION ............153

6.3. Characterizing Attentive BehaVior in InTELLIgent EnVIRONMENTS ........................... 154

6.4. .. MODELLING A SMART ENVIRONMENT FOR NONINTRUSIVE ANALYSIS OF ATTENTION IN THE WORKPLACE

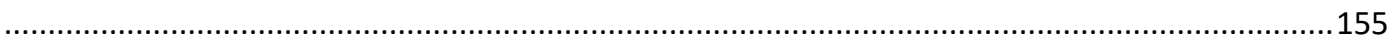

6.5. PREDICTING COMPLETION TIME IN HIGH-STAKES EXAMS ........................................... 156

7. Conclusions and Future Work .................................................................... 157

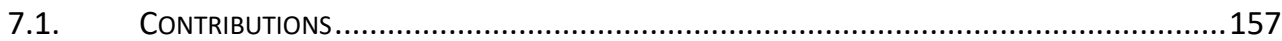

7.2. VALIDATION OF THE RESEARCH HYPOTHESIS........................................................ 162

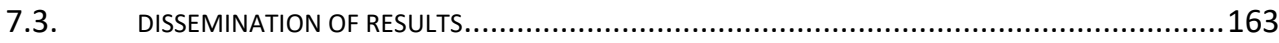

7.3.1. Other Publications ..................................................................... 163

7.3.2. Participation and Organization of Events ............................................ 167

7.3.3. Supervision of Students............................................................. 167

7.4. FINAL REMARKS AND FUTURE WORK CONSIDERATIONS .......................................... 168

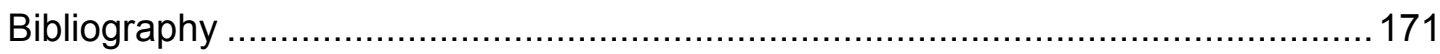




\section{LF}

\section{LIST OF FIGURES}

Figure 1.1 - Activities competing with the School............................................ 28

Figure 1.2 - Students deal with constant notifications ............................................ 31

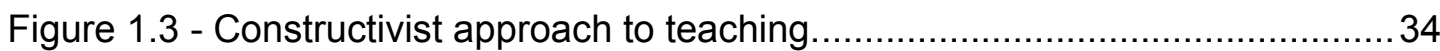

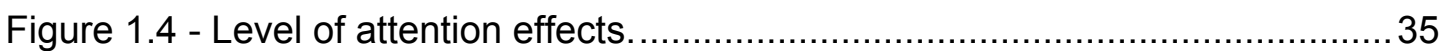

Figure 2.1 - The six basics emotions (Ekman, 1992) .........................................46

Figure 2.2 - The circumflex model of affect (Russell, 1980)..................................47

Figure 2.3 - Watson's two-dimensional structure of affect (Watson, Wiese, Vaidya, \&

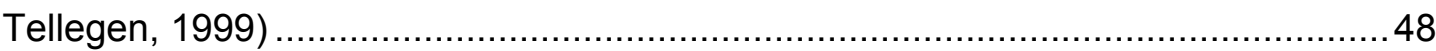

Figure 2.4 - OCC Model (Ortony, Clore, \& Collins, 1990) .................................... 50

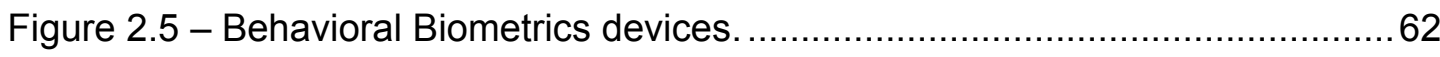

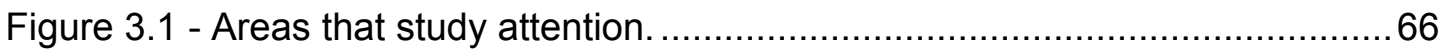

Figure 3.2 - BroadBent's model (McLeod, 2008) ..............................................69

Figure 3.3 - Conceptualization of the set of factors that influence attention level. ....72

Figure 3.4 - A generic diagram for representing the multi-modality space in the recognition stress model (Rodrigues, 2013). ................................................... 74

Figure 3.5 - Engagement States and Engagement (Deater-Deckard, Chang, \&

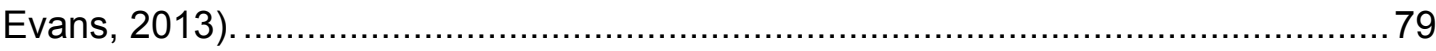

Figure 3.6. - Engagement model and its attributes (O'Brien \& Toms, 2008)............. 82

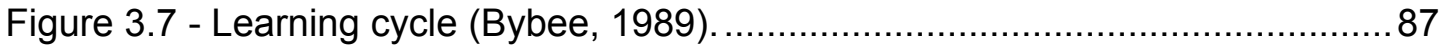

Figure 3.8 - Kolb Learning Cycle (Kolb D. , 1984). ............................................... 91

Figure 3.9 - Kolb's Learning Style (Kolb \& Kolb, 2005) .................................... 92 
Figure 3.10 - Honey and Mumford learning model (Honey \& Mumford, 2006)........99

Figure 4.1 - Attention as form of engagement............................................ 102

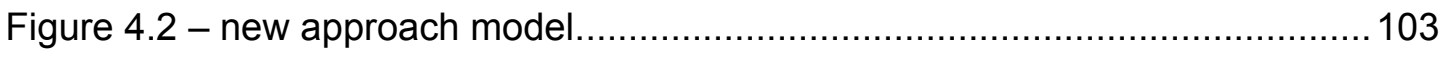

Figure 4.3 - Dynamic Framework of attention analysis. ................................. 105

Figure 4.4 - Proposed framework .............................................................. 108

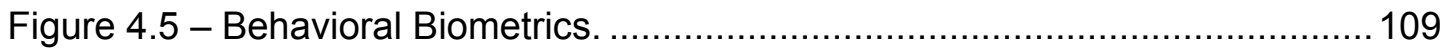

Figure 4.6 - data result of mouse and keyboard interaction.............................. 112

Figure 4.7 - (A) A series of mouse movements events (MV), between two consecutive clicks of the mouse. The difference between the shortest distance (sdist) and distance actually travelled by the mouse (rdist) is depicted; $(B)$ The real distance travelled by the mouse between each two consecutive clicks is giving by summing the distances between each two consecutive MV events; (C) The sum of the angles of the mouse's movement is given by summing all the angles between each two consecutive movement vectors; (D) The average distance at which the mouse is from the shortest line between two clicks is depicted by the straight dashed line

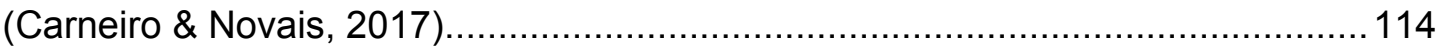

Figure 4.8 - Client Visualization selecting work task-related............................115

Figure 4.9 - Sequence of applications used by a specific student, with the date in which the student switched to other application and the time spent interacting with it.

Figure 4.10 - Mouse Behavior Biometrics of two class. .................................. 116

Figure 4.11 - Example of a Keyboard TBK of two classes. ................................. 117

Figure 4.12 - Real-time performance: evolution of one student's interaction performance during the exam. Left: Click Duration. Right: Mouse Velocity............118

Figure 4.13 - Detail of evaluation of attention for a specific student (Durães D. , Carneiro, Bajo, \& Novais, 2016) .............................................................. 121

Figure 4.14 - Data set example................................................................. 123

Figure 4.15 - K-means algorithm process example (Anil, 2010) ..........................125

Figure 5.1 - Distributions of the data collected in the two different classes (Evaluation vs. Normal), in four different features: Mouse Velocity, Mouse Acceleration, Click Duration and Distance of the Pointer do the Line Between Clicks........................132

Figure 5.2 - Comparing mv, ma, and cd during the lessons. ............................. 133

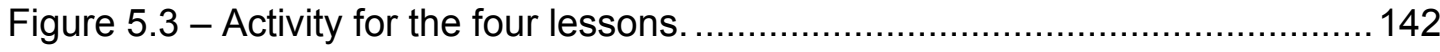

Figure 5.4. - Feature Selection - Boruta Method. .......................................... 142 
Figure 5.5 - Real-time performance: evolution of one student's interaction performance during the exam. Left: Click Duration. Right: Mouse Velocity............ 145 Figure 5.6 - Random Forest: Error vs Trees based on the different labels/activities 148

Figure 5.7 - Random Forest: Features Relevance in Activity Classification 148 


\section{LT}

\section{LIST OF TABLES}

Table 2.1. Emotions (Shaver, Schwartz, Kirson, \& O'Connor, 1987) ......................58

Table 3.1. Summary of the engagement attributes (O'Brien \& Toms, 2008)........... 81

Table 3.2. Some definitions of learning (Pritchard, 2009) ...................................... 83

Table 3.3. Comparison between Honey and Mumford model and Kolb model.........94

Table 3.4 - Felder-Silverman Learning Styles and Teaching Style (Felder \&

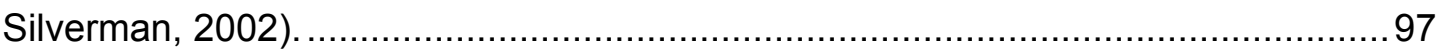

Table 4.1 - General view of dataset of behavioural biometrics. ...........................110

Table 4.2 - mouse movement example. .................................................... 111

Table 4.3 - Relation between type of tasks performed and the learning style theory in

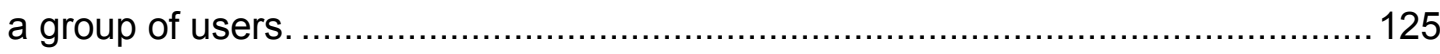

Table 4.4 - Comparative Analysis of Machine Learning Categorisation Performance.

Raw Min-Max Normalisation Z-score ........................................................ 127

Table 5.1 - Results of the Kruskall-Wallis test and mean values for ech class and each feature.

Table 5.2 - Total time (seconds) devoted to the task and percentage of total time devoted to the task, while being assessed and while in a normal class, for each student. 134

Table 5.3 - Classification for keyboard, mouse and tasks 135 Table 5.4 - The average interaction of the mouse and the keyboard of the class for this lesson 137 
Table 5.5 - The amount of time that each student spent interacting with the computer and the amount of actual work versus the amount of time spent interacting with other

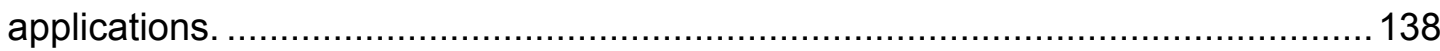

Table 5.6 - Relative attention of each user in the lesson. .................................. 138

Table 5.7 - Summary of the characteristics of each assess activity......................139

Table 5.8 - Summary of the biometric features variation for each exam................ 141

Table 5.9 - Summary of the characteristics of each exam................................ 143

Table 5.10 - Comparative Analysis of Machine Learning Categorisation

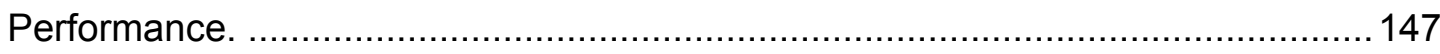

Table 5.11 - Random Forest: Confusion Matrix.................................................. 149

Table 6.1 - Conference paper PAAMS 2016 ................................................ 152

Table 6.2 - Conference Paper IE 2016 ....................................................... 153

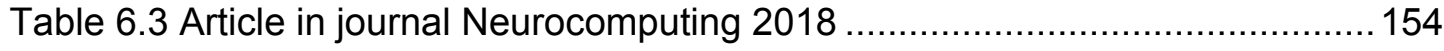

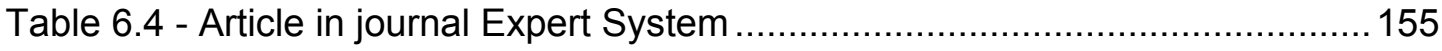

Table 6.5 Article in journal Future Generations Computer Systems ......................156

Table 7.1 - Reply to the initial Objectives...................................................... 161 


\section{LE}

\section{LIST OF EQUATIONS}

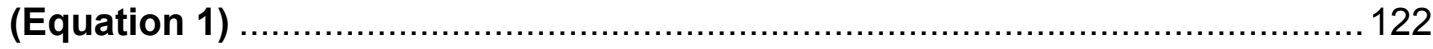

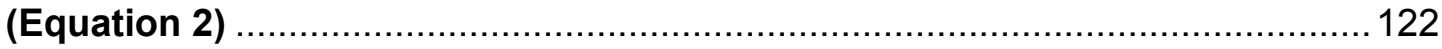

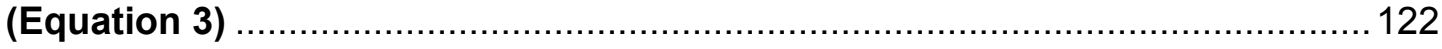





\section{LA}

\section{LIST OF ALGORITMS}

Algorithm 4.1 - Creating triplets with the durations and timestamp of each application (Carneiro, Durães, Bajo, \& Novais, 2016)... 119

Algorithm 4.2 - Creating triplets at regular intervals with the timestamp and the quantification of attention (Carneiro, Durães, Bajo, \& Novais, 2016).....................120 Algorithm 4.3 - Learning Style Process...........................................................126 



\section{Ac}

\section{ACRONYMS}

A

AC - Affective Computing

Al - Artificial Intelligence

Aml - Ambient Intelligent

ANEW - Affective Norms for English Words

B

BPMS - Body Posture Measurement System

E

ECG - Electrocardiogram

EDA - Electro Dermal Activity

EEG - Electroencephalography

EMG - Electromyograms

EOG - Electrooculogram

ERP - Event-Related Potential 


\section{F}

FER - Facial Expression Recognition
fMRI - Functional Magnetic Resonance Imaging

G

GBS - Goal-based Scenarios

I

IADS - International Affective Digitized Sounds

IAPS - International Affective Picture System

IE - Intelligent Environments

L

LIWC - Linguistic Inquiry and Word Count

$\mathbf{M}$

MDS - Multidimensional Scaling

MEG - Magnetoencephalography

$\mathrm{MHCl}$ - Multimodal Human-Computer Interaction

MMN - Mismatch Negativity

MRI - Magnetic Resonance Imaging

$\mathbf{P}$

PET - Positron Emission Tomography

S

SER - Speech Emotion Recognition

SCRUM - a framework for developing and sustaining complex products. 
TDCS - Transcranial Direct Current Stimulation

TEL - Technology-Enhanced Learning

TMS - Transcranial Magnetic Stimulation

$\mathbf{Z}$

ZPD - Zone of Proximal Development

Number

3S's - Seamless Assistance Supervising 

"A hypothesis is a novel suggestion that no one wants to believe." - Edward Teller

\section{1}

\section{INTRODUCTION}

We live in a global, multicultural, and hyper-connected world where technology is present in all spheres of life and is the backbone for the transformation of our society, which is in permanent change and requires continuous adaptation of the human being to the surrounding environment (Durães D. , Carneiro, Bajo, \& Novais, 2016).

In a traditional learning environment student and the teacher are the key elements in the classroom. In environments in which technologies exist, the teacher plays an essential role in providing an engaging learning and teaching environment. Together, teacher and students take a set of physical, social, emotional, mental characteristics, and needs to the classroom. These features influence the way one relates to the other and consequently affect the way the learning process will progress. The teacher is able to learn about necessities and educational philosophy as well as the nature of his/her students; this will improve the teacher's position to facilitate student's learning. Teacher-student relationships are a crucial aspect of the learning success, and it is one of the key aspects that helps determine the success or failure of a teacher's work. Relationships that are considered positive, concerning respect and empathy, emphasize all the students' abilities and engage them throughout the learning process (Hamre \& Pianta, 2006).

In most of the so-called developed countries, students have nowadays increasingly busier activities. This makes them stretch their limits to find time for 
classes, homework's, sports activities and other activities. An example is presented in Figure 1.1. The necessary extra time is frequently obtained at the expense of shorter periods of sleep or rest, and with a cost in terms of pressure and stress (Pimenta, Carneiro, Neves, \& Novais, 2016). Although this effect may not be readily visible, they have consequences at many other levels than health, including emotions, results, attention, and social behavior, among others (Folkard \& Tucker, 2003).

For these reasons, the school must create systems that are capable of involving students and capture their attention. Advances in computers and wireless technologies have also had an impact on the educational setting, thus generating a new approach for Ambient Intelligent Systems (Aml systems). The rapid development of these technologies combined with the access to content in a wide variety of settings, allows learners to experience new learning situations beyond the school's walls.

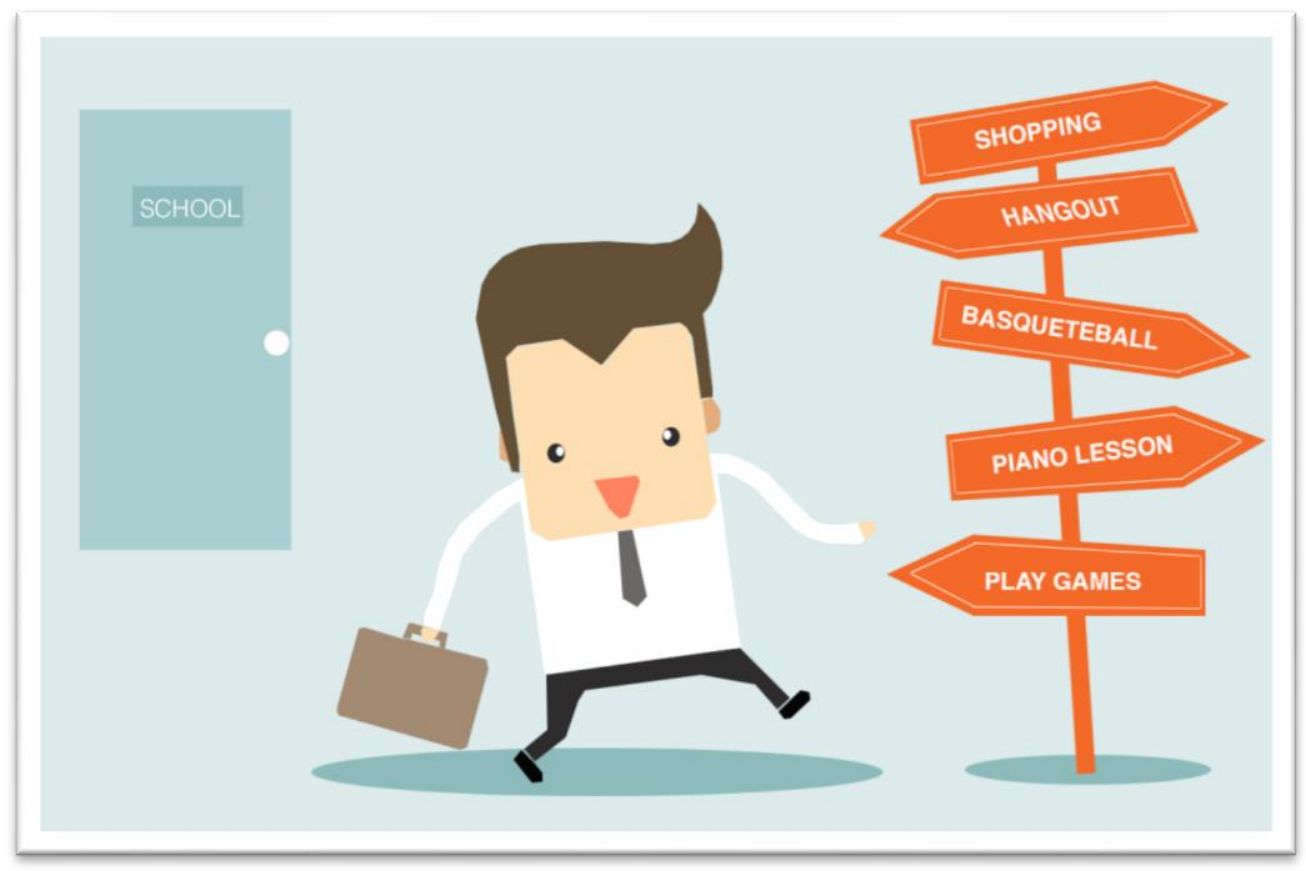

Figure 1.1 - Activities competing with the School.

In the last years, the school has introduced computer-based training or networked based learning in order to improve the field of Aml systems (Neto, Gauthier, \& Nassar, 2005)]. These systems must have the following features: context awareness and adaptively context. The definition of context awareness denotes that 
the pedagogical progress and the context that is provided to the learning environment should be aware of the situations in which the learner actually is. The adaptively context refers to the idea that different learning context should be adaptable to the particular setting in which the learner is situated (Milrad, et al., 2013).

Being a cognitive process, attention is strongly connected with learning (Pimenta, Gonçalves, Carneiro, Riverola, \& Novais, 2015). When it comes to acquiring new knowledge, attention can be considered one of the most important mechanisms (Mancas, Ferrera, Ritche, \& Taylor, 2016), where the level of the learner's attention affects learning results, and the lack of attention can define the success of a student.

The use of the new technologies in the context of the classroom caused a great change in the learning concept. From this new situation, the problem arose of the amount of attention that the student has in a class (Treisman \& Gelade, 1980). The idea will be to quantify the level of attention. The definition and quantification of such measure is the key element in this thesis, provided through real-time analytics (Kejariwal, Kulkarni, \& Ramasamy, 2015). It is especially suited for milieus where students work with the computer for long periods of time. It is designed with the purpose to provide the teachers with important knowledge about each individual behavioral throughout the day varies and to informed the teacher how each individual reacts to events (e.g. increased/decreased attention and engagement).

\subsection{Motivation}

The development of new learning environments, supported by technology, may allow to improve the learning process but it is also necessary to mitigate problems that may occur in an environment with learning technologies. Learning theories provide insights into the very complex processes and factors that influence learning and give precious information to be used in designing instruction that will produce optimum results. The learning models are designed in order to supply the students with practice, evaluation and improvement procedures which will adjust the model (Eggen \& Kauchak, 1992). 
However, for various reasons, students may not be predisposed to learning. Moreover, each student has its own particular way of assimilating knowledge, that is, his/her learning style. In this sense, and in bigger classes, it is important that the instructor/teacher has instruments to point out potential distractions (namely in what concerns the applications being used by the students) that may indicate a lack of predisposition to learning.

Nowadays, we have to deal with constant notifications from our e-mail, our social networks, our messaging applications, advertisements and so on. We live immersed in beeps, vibrations, notifications and blinking icons, which constantly call for our attention and distract us (Gottlieb, 2012). Even if we return immediately to our task, the fact that we had to consciously evaluate the stimuli to decide that it is not important at the moment already had a toll on our brain, making it spend resources (Davenport \& Beck, 2013), ( Simola, Hyönä, \& Kuisma, 2015).

This is especially worrying in young children, who nowadays have a facilitated access to computers, mobile phones, and tablets, with their games and engaging applications (see in Figure 1.2). For them it is so easy to get distracted by these stimuli, making learning less efficient and more frustrating, negatively affecting their development ( Gottlieb, 2012).

Currently, one of the major problems related to learning is the quantity of attention that a student spends on performing a proposed task. The level of attention of each person is increasingly affected by the evolution of Internet usage and the introduction of social networks. These two factors have a high impact on attention because it offers many information of general interest and might cause inattention.

Attention is a very complex process through which one individual is able to continuously analyze a spectrum of stimuli and, in a sufficiently short amount of time, and to choose one to focus on. In most of us, who can only focus on a very reduced group of stimuli at a time, this implies ignoring other perceivable stimuli and information.

Another important aspect is emotions. Emotion plays an important role in the decision process and knowledge acquisition of an individual. Therefore, it directly influences perception, learning process, the way people communicate, and the way 
rational decisions are made. So the importance of understanding affects and its effect on cognition and in the learning process. To understand how emotions influence learning process several models were developed. Models like: Russell's Circumplex Model (Russell, 1980) are used to describe the user's emotion space; and Kort's Learning Spiral Model (Kort, Reilly, \& Picard, 2001) are used to explore the affective evolution during the learning process.

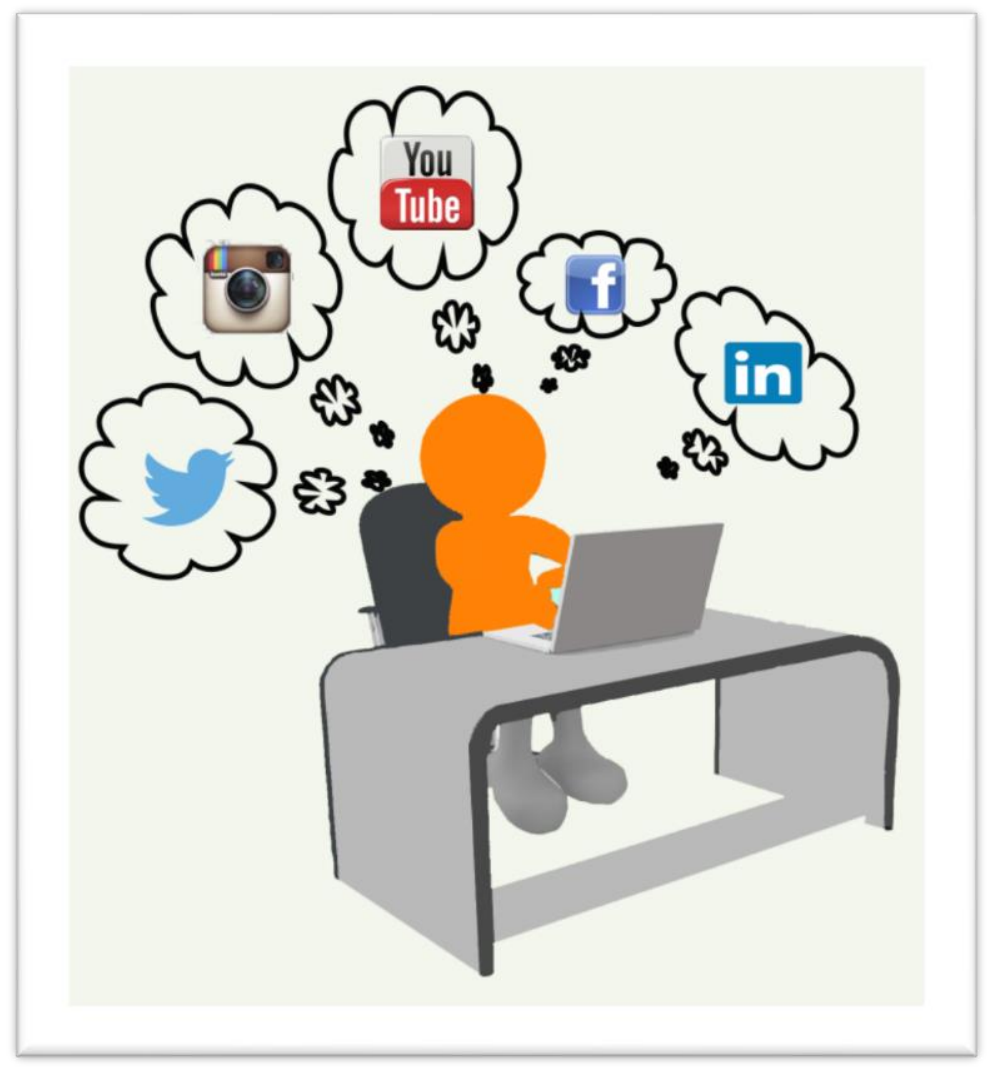

Figure 1.2 - Students deal with constant notifications.

It is crucial to improve the learning process and to mitigate problems that might occur in an environment with learning technologies. Besides, each student has his/her own particular way of assimilating knowledge, that is, his/her learning style. Learning styles specify a student's own way of learning. Someone that has a specific learning style can have difficulties when submitted to another learning style.

This research deals with the issue of attention monitoring, with the aim of providing a non-intrusive, reliable and easy tool that can be used freely in schools or organizations, without changing or interfering with the established working routines. Specifically, we look at desk students in learning activities, in which the student 
spends a long time interacting with the computer. So, we want to create a distributed system for monitoring attention in student classroom, in line with the vision of Intelligent Environments (Augusto, Callaghan, Cook, \& Kameas, 2013). The idea is to detect a lower level of attention and indicate this situation to the teacher. Consequently, the can act in real-time in order to improve learning.

\subsection{Challenges of Teaching}

Teaching process has few steps that it is necessary to follow. Firstly, it requires that the instructor/teacher creates a pedagogical design of the objectives and determines the content to be taught. Secondly, a pre-assessment is used to determine learning abilities. Thirdly, pedagogical procedures are used when teaching is initiated. Finally, assessment is applied to determine what learners have achieved, and, according to the assessment results, instructors/teachers should use feedback to determine the cause of ineffective instruction (Eggen \& Kauchak, 1992), (Hopkins, 1998).

Teaching should be solidly grounded in the absolute understanding of how the process of learning occurs so that instructional strategies could be efficient and lead to persistent knowledge. This is especially true when learning activities involve technologies (Gonçalves, Rodrigues, Carneiro, Fdez-Riverola, \& Novais, 2015). In such cases, some of the previously mentioned issues can negatively affect the acquisition of knowledge and it is necessary that the teacher engages the students, because they have other technologies and applications that they can use and this can distract them.

The concept of "big class" may, however, differ according to the context. In Europe, for example, a class with 50 students could be considered a big class. However, in China, big classes might have 150 students (Xu, 2001). In classes with

these dimensions, the instructor/teacher has difficulties in assessing the student's commitment to the tasks during the class.

In this sense, and in bigger classes, it is important that the teacher has instruments to point out potential distractions (namely in what concerns the applications being used by students) that may indicate a lack of predisposition to learning. Someone that has a specific learning style can have difficulties when 
submitted to another learning style (Smith \& Renzulli, 1984). When the given instruction style matches the student's learning style, the process is maximized which guarantees that the student learns more and more easily.

Technologies that enhance learning environments are ideal for generating learning style-based instructional material in large classes, as they do not have the same limitations as human instructors due to the lack of resources and time to focus on individual students. With this recommendation, the teacher can improve some strategies that may increase the level of attention and engagement of the students and they might improve learning (Andrade, Novais, Carneiro, Zeleznikow, \& Neves, 2009).

Education, training, skills development, and learning are processes that are continuously performed since we are born. These characteristics and the ability to learn and teach, allow us to grow as a person. Besides, learning is inevitably linked to the History of Humanity, to our construction as a social being capable of adapting to new situations. The learning process is described as the act of acquiring knowledge, behaviors, abilities, standards or preferences and the study of learning has been closely linked to the development of psychology as a science (Carroll, 1997).

Many contemporary educators argue the value of a constructivist approach to teaching. Advocates argue that constructivist learning better equips learners to successfully master news and novel situations (Clark, 1983). It emphasizes problemsolving over the mere accumulation of facts. One of the central arguments for the use of Web-based resources in the classroom is that it gives learners access to information resources in ways that allow them to search for relevant data, synthesize that information, and draw their own conclusions (See Figure 1.3.).

For these reasons, school must create systems that are capable of involving the students and capture their attention. The concept of adaptively refers to the idea that different learning contexts should be adaptable to the particular setting in which the learner is situated (Milrad, et al., 2013). 


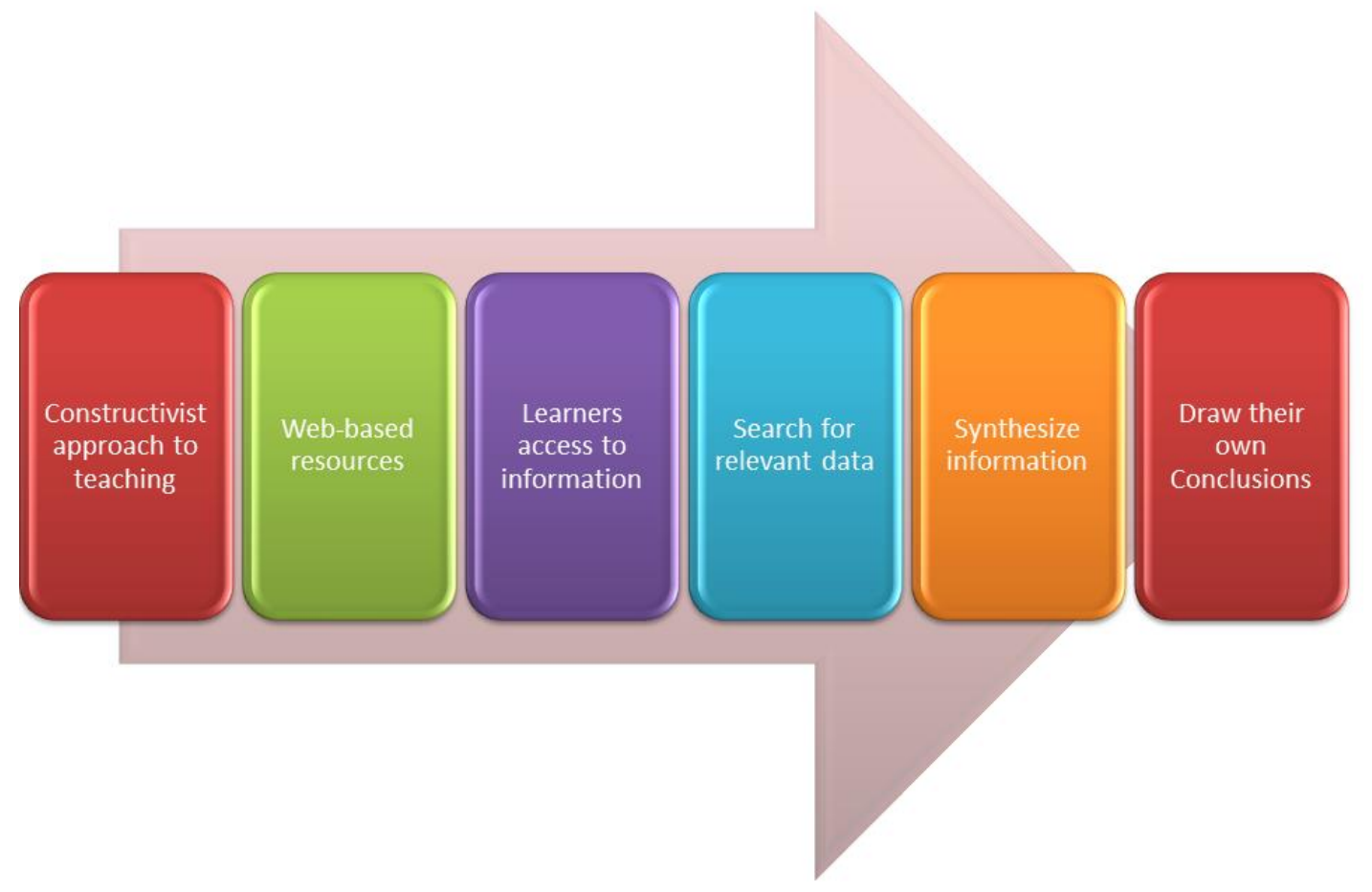

Figure 1.3 - Constructivist approach to teaching.

\subsection{ATtentivenESS AND ENGAGEMENT}

Attention is the cognitive process of focusing on one aspect of the environment while disregarding others (Mancas M. , 2015). "Pay attention!" is a phrase repeated by so many teachers all over the world, and the explanation for this is simple (James, 1890). Attention is the first step in the learning process. Students cannot learn or understand or even remember if they do not listen properly, if this situation occurs, they will fail in the learning process. For almost everyone it is easy to pay attention to subjects or things that are interesting or stimulating to them. In most of us, that can only focus on a very reduced group of stimuli at a time, it implies ignoring other perceivable stimuli and information (Mancas M. , 2015).

Although these aspects have always existed, in recent years we have observed an increase in stimuli that make students distracted, namely in classroom, examples are use of the Internet, online games and social networks. We can then say that this topic of attention has come to play a crucial role in learning. The 
student's level of attention affects learning outcomes. Lack of attention can define a student's success. In the learning activities, the level of attention that the student dispenses is very important to carry out the tasks defined in an efficient and appropriate manner. Figure 1.4 presents a brief summary of learning evolution and its relationship to attention.

Research on attention involves nowadays many fields, including education, psychology, neuroscience, cognitive neuroscience, and neuropsychology. For this reason, many different views and theories on attention can be found. One of the most frequent ones is the so-called Attention Economics, which treats human attention as a scarce commodity or resource, which we must use wisely in order to attain our goals (Davenport \& Beck, 2013). Individuals who have difficulty focusing their attention can see the performance of negatively affected cognitive processes, such as learning or decision making. In extreme cases, such as in AttentionDeficit/Hyperactivity Disorder (ADHD), this may have a significant negative impact in the development and function of the individual (ATTENTION-DEFICIT \& et al., 2011).

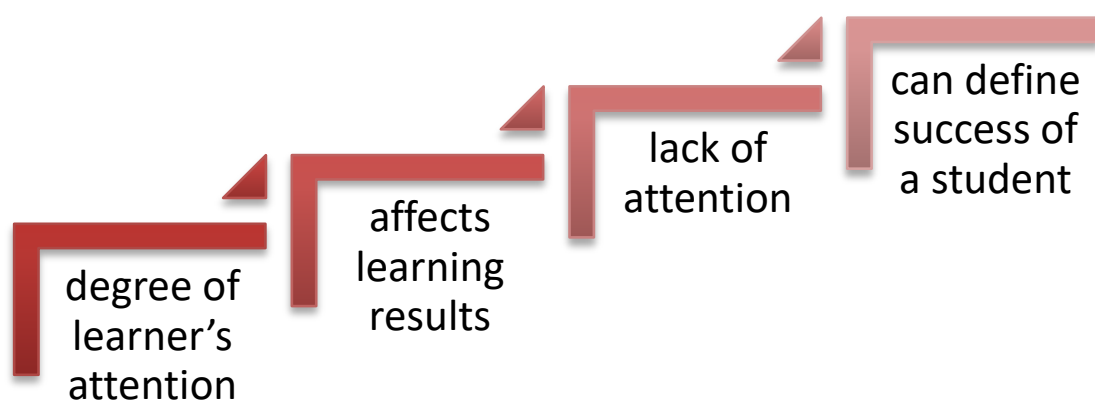

Figure 1.4 - Level of attention effects.

On the other hand, engagement is one of the most widely misused and overgeneralized constructs found in the educational, learning, instructional, and psychological sciences. As the opposite of detachment, engagement is rarely mentioned in the psychological literature. However, Ortony (Ortony, 2002) used the term "caring" to express what seems to be a related concept. Nerveless it does concern people working and being materially engaged with the virtual surroundings, rather than essentially distanced from them. In that context, it is widely assumed that 
full emotional responsiveness to virtual surroundings implies and depends on engagement with them (Huang \& Alessi, 1999).

In that sense, engagement has been used to describe everything that involves people, including: student academic performance and achievement; classroom behaviors; approaches to interacting with instructional materials; students' self-perceptions of beliefs in handling individual and contextual aspects of learning situations; students' enactment of cognitive, motivational, affective, metacognitive, and social processes, particularly in academic contexts (e.g., classrooms, intelligent tutoring systems); teacher practices in learner-centered classrooms; and features of instructional and learning contexts designed to initiate, sustain, and foster learning (Azevedo, 2015).

Researchers who work closely with educational, learning and psychological are also adopting and using engagement to refer to motivational beliefs, behavioral enactments of cognitive strategies, affective states, persistence, and self-regulation (Azevedo, 2015).

\subsection{RESEARCH HYPOTHESES}

Taking into account the challenges of learning, attention, and engagement, which were pointed out in Section 1.2 and Section 1.3 there should be solutions to address them. The objective of any computational system should be to enhance learning and help the teacher to improve the learning of his/her students. However, at this moment no system with these characteristics exists.

The goal of this thesis is, thus, to answer this challenge by turning to Al techniques such as affective computing specification, logic programs, and machine learning to outline a framework. So, in this work is proposed the architecture of an ambient intelligent system aimed at capturing and measuring the level of the students' attentiveness in real scenarios, analyzing the student's interactions with the peripheral devices, and dynamically provide recommendations to the teacher. The students can learn in a relatively anonymous environment without the embarrassment of failure and/or socio-cultural prejudice from personal contact. All the students get the same standardized set of learning materials from the learning activities using technologies in an online environment. It is especially suited to a 
student working with computers and can be interesting for domains such as the workplace or the classroom. It constantly analyzes the behavior of the user while interacting with the computer and, together with knowledge about the task, is able to predict the user's behavior.

This work should be very interesting: (1) for the teacher in the schools to assess the behavior profile of their classroom, identifying potentially distracting events, hours or individual's situations. Moreover, distraction often appears when the user is fatigued, bored or not motivated; (2) In the workplace, this tool can thus be an important indicator of the team, allowing the manager to act accordingly at an individual or group level; (3) In the overall, this tool will support the implementation of better human resources management strategies.

So, the research hypothesis for this work is formulated as follows:

"It is possible to analyses attention and engagement in students learning activities by and non-invasive approaches, from the observation of changes of an individual or a class, concerning behavioral pattern in order to predict students behavioral in (e)learning lessons.".

\subsection{OBJECTIVES}

The title of this doctoral thesis is "Attentiveness and Engagement in Learning Activities". In that sense, the developed work focuses on the matters of knowledge representation of attention and engagement reasoning with this knowledge, and the underlying decision-making process.

Taking as a starting point the user attention the objective of this doctoral thesis lies in the study of the main aspects of the creation and editing tools of an intelligent system that monitors attention and creates a user behavior pattern.

For this we identify central research questions that consist of:

$\checkmark$ How to detect attention?

$\checkmark$ What methods are needed to detect attention?

$\checkmark$ Why is it necessary to classify attention?

$\checkmark$ Where is it essential to classify learning styles? 
$\checkmark$ When it is crucial to characterize the student's decision-making?

$\checkmark$ Who can uses the create predict model?

The research questions previously specified allowed us to approach in terms of the following objectives:

1. Monitor students' biometric behavior, and activity performance during lessons.

2. Characterize student's mouse dynamics, decision-making, and attention profile.

3. Test the hypothesis that mouse dynamics and keyboard dynamics can predict student's attention level.

4. Test the hypothesis that is possible to predict attention level based in different applications of learning style.

5. Create adequate prediction and classification model based on emotion affects.

6. Apply the prediction model on (e)learning platforms for non-invasive monitoring.

\subsection{RESEARCH METHODOLOGY}

To develop this doctoral thesis, the Action Research Methodology (O'Brien, 1998) was employed. It is a holistic approach to problem-solving, based on continuous development, and directed to problem-solving. What distinguishes this approach from normal, everyday problem-solving is the emphasis it puts on scientific study. The researcher systematically studies the problem and his interventions are supported by theoretical considerations. The process starts with a research hypothesis, based on a thorough review of the state of the art, in this case, affective computing and attention as a form of engagement. From this step results a report of available resources and features. In the context of this doctoral thesis, and faced with what was exposed in Section 1.3, the hypothesis is defined in Section 1.4. After the formulation of this hypothesis, a set of objectives is established for this work and are outlined in Section 1.5. The Action Research has six steps modelled as follows: 
1. Diagnosing - Definition of the problem and issues: this step states the problem and investigates what originates it, gathering all its features and formulating a hypothesis;

2. Action planning - A constant update of the state of the art: the review of the state of the art studies of all features of the projects related to the current research. The update of this component allows the adjustment of objectives and the pursuit of new solutions;

3. Action taking - Development of a prototype with the information gathered in the previous steps enables the design of a solution that fulfils the specified objectives, which, in turn, will allow proving or refuting the research hypothesis;

4. Evaluation - Implementation and experimentation through prototypes: formalization of a prototype that contains all features specified in the solution. Its behavior is then observed in order to verify its efficacy;

5. Validation - Analysis, validation and conclusion: this step consists of the analysis and validation of the prototype. This is done by checking if the implementation achieves the objectives, which leads to conclusions regarding the research hypothesis;

6. Learning dissemination - Dissemination of results in the scientific community: dissemination of research results in peer-reviewed journals, conferences, workshops, among others.

As for the development of software solutions, the methodology used will be adapted from SCRUM. Thus, all previously explained steps will be applied in software development.

The first steps are diagnosing the problem and updating the state of art and objectives of the work. Next is the development of the software of the proposed objectives. When these tasks are completed, an evaluation of the work will be done. Through these results, new problems arise which leads to a new cycle.

SCRUM development is a simple methodology intended to solve long product development, which allows the developer to focus on the set of goals proposed. This methodology also solves the mismatch problem between a product's business 
requirement and the actual resulting implementation (which normally occurs when developing big products).

\subsection{STRUCTURE OF THE THESIS}

This section aims to be a guide for helping readers to easily find the right content of this thesis and, also, to understand some aspects related to the structure of the document. This thesis is presented under the scheme of a book. The first content to be provided is a summary of the presented doctoral thesis, both in English and Spanish. This is followed by a list of Keywords, the necessary Table of Contents, List of Figures, List of Tables, List of Equations, List of Algorithms, and Acronyms. The thesis can be divided into seven main blocks: Introduction (Chapter 1), Affective Computation (Chapter 2), Attention as form of Engagement (Chapter 3), 3S's Architecture (Chapter 4), Analysis of Practical Results (Chapter 5), Publications composing the Doctoral Thesis (Chapter 6), and Conclusions and Future Work (Chapter 7). At the end of the thesis, the Bibliography used is presented.

Each chapter of the thesis has a well-defined purpose. As such, a brief summary of each one is presented. The description is as follows:

\section{Chapter 1: Introduction}

This chapter introduces the thesis with the exposition of concepts that are relevant to the understanding of its scope and purpose. It is described a brief description of the current situation, an introduction to key concepts and a presentation of motivation, and limitation. Based on these limitations and the challenges it presents, the research hypothesis and the objectives for the thesis are defined. Following this, the research methodology is outlined. The section ends with a document guide describing the structure and contents of the thesis.

\section{Chapter 2: Affective Computation}

In the second chapter, are discussed all concepts related to emotions. So are presented the concepts of: sensing and recognizing emotions; affective states, and affective learning; and user behavior. In all of these concepts are showed the theoretical foundations of each concept. 


\section{Chapter 3: Attention as form of Engagement}

This chapter is divided into four important approaches: attention, engagement, learning, and learning styles. Like in the Chapter 2, all concepts are presented based on the theoretical foundations. Several theories are presented and at the end it is presented a summary of the chapter.

\section{Chapter 4: 3S's Architecture}

The fourth chapter outlines the project and system architecture designed and developed, where are specified the technologies used and their implementation details.

\section{Chapter 5: Analysis Practical Results}

In chapter fifth are described some case studies based on the research objectives, the dataset, and the data collection method. It also includes all forms that data were analysed, and the results are explained.

\section{Chapter 6: Publications composing the Doctoral Thesis}

The publications featured in this Chapter are the most important publications of this work, which were published in scientific journals in the field of this research.

\section{Chapter 7: Conclusions and Future Work}

In the Conclusions and Future Work chapter, there is a description of the contributions resulting from the thesis and how they answer to the initial objectives. It is also presented how the research hypothesis is validated. In addition, the activities undertaken for the dissemination of results are enumerated. The chapter ends with final remarks and considerations about future works. 
"Behavior is the mirror in which everyone shows their image." - Johann Wolfgang von Goethe

\section{AFFECTIVE COMPUTING}

The concept of Affective Computing (AC) was introduced by Picard (Picard R. , 1997), who defined it as: "the computing that relates to, arises from, or deliberately influences emotions". Lee and Park (Lee, Choi, Lee, \& Park, 2012) also defined AC as the study of technologies to recognize, model, and express human affective phenomena such as emotion, moods, attitudes, and personality traits. The AC focus on establishing models, based on physiological and behavioral signals collected by sensors and techniques to perceive, recognize, and understand human emotions and provide better feedback.

According to Hassin, Aziz and Norwawi (Hassin, Aziz, \& Norwawi, 2004), the appearance of $A C$ is related to the needs to put computers interacting directly, thinking, receiving, and transmitting people's personalities. The authors Picard and Hassin (Picard R. , 1997) (Hassin, Aziz, \& Norwawi, 2004) emphasize AC as a research area, which examines how computational systems can identify, classify, and prove human personality. In addition, it aims to be a group of knowledge for other areas, such as psychology and cognitive sciences.

$\mathrm{AC}$ also has a great potential as a subcomponent technology for other systems. AC can enhance the capabilities of customer relationship management and recommendation systems. Likewise, it can be exploited for affective mentoring and affective entertainment or for filtering and detecting spam in communication on online social networks. 
There are several fields in which $\mathrm{AC}$ can be applied: $\mathrm{HCl}$; text-based communication, and virtual reality; monitoring humans; and education systems. In the field of $\mathrm{HCl}$, the machine needs to sense and react respectfully to human emotion. For instance, if a person is communicating with a technology and he/she is feeling frustrated or confused, the technology needs to be able to respond differently to that person. In the field of text-based communication, and virtual reality there are systems: that analyse if the text has a positive or negative emotion (Pang \& Lee, 2006), and detects emotion through user's communication and expresses this emotion in virtual environments. In the field of monitoring humans, there are some applications like call centers, elderly care, and surveillance systems where the system should react differently to different situations and persons. Finally, the field of education systems will be described in subsection 2.4 .

$A C$ in part about understands how emotions play vital roles in us, regulating our intention, helping us make good decisions, changing the way we emphasize and prioritize things, organizing or figuring out what matters. With the purpose of increasing the quality of human-computer communication, and also improve the intelligence of the computer, AC builds an "affect model" based on the various sensors-captured information. Thus it is possible to build a personalized computing system with the capability of perception, interpretation of human's feelings as well as giving us intelligent, sensitive and friendly responses.

\subsection{SENSING EMOTIONS}

So emotion plays an important role in the knowledge, acquisition, and decision process of an individual. Consequently, they directly influence perception, learning process, and the way people communicate. There is also significant evidence that rational learning in humans is dependent on emotions (Picard R. , 1997).

Psychology, cognitive science, neuroscience, engineering, computer science, sociology, philosophy, and medicine are fields that study affects. All of these different studies have contributed to a different understanding of basic terms related to affects such as emotions, feelings, moods, attitudes, affective styles, temperament, motivation, attention, reward, and so many others (Picard, Papert, Bender, \& 
Blumberg, 2004). One of the problems in studying affects is the definition of what emotion is.

To analyse emotion, there are several theories, which attempted to specify the interrelationships of all the components involving an emotion and the causes, the reasons, and the function of an emotional response. In that sense, almost a hundred of definitions of the term emotions have been registered since 1981 (Kleinginna \& Keinginna, 1981).

Some research intended to relate emotion and computer, so for Ortony , Clore and Collins (Ortony, Clore, \& Collins, 1990) emotion identification is generally used in the field of cognitive science has a connection to affective computing enabling computers to recognize and express emotions (Picard R. , 1997).

Many $\mathrm{AC}$ and $\mathrm{HCl}$ researchers have suggested various methods to sense and recognize human emotions (Rincon, Costa, Villarrubia, Julian, \& Carrascosa, 2018). We express our emotions in two main ways: the modulation of facial expression (Ekman, 1992) and the modulation of the intonation of the voice (Banse \& Sherer, 1996).

Historically, several models have been created in an effort to systematize the emergence of emotions, their associated behaviors and to discuss how emotions are occasioned in our cognitive system. Nevertheless, the main research theories are the discrete, the dimensional and the appraisal ones.

\subsubsection{DISCRETE EMOTIONAL THEORIES}

Discrete emotional theories propose the existence of basic emotions or states (happiness, anger, sadness, surprise, disgust, and fear, for instance) that are universally displayed and recognized (Ekman \& Friesen, 1969), (Keltner \& Ekman, 2000). Discrete models tend to group emotions into categories and assume that they are independent. In the literature, among the discrete models, the most known is the model of basic emotions, proposed by Ekman (Ekman, 1992). This model proposes the existence of six basic emotions: happiness, sadness, fear, anger, surprise and disgust. One of the main advantages of discrete models is that, through psychophysical experiments, the perception of emotions by human beings is discrete. This way, these models can easily associate emotions with the facial 
expressions that represent them. The six basic emotions of Ekman (Ekman, 1992) are represented by six universal facial expressions, since they are understandable by people in different locations, regardless the culture. The facial expression of the six basic emotions is shown in Figure 2.1.

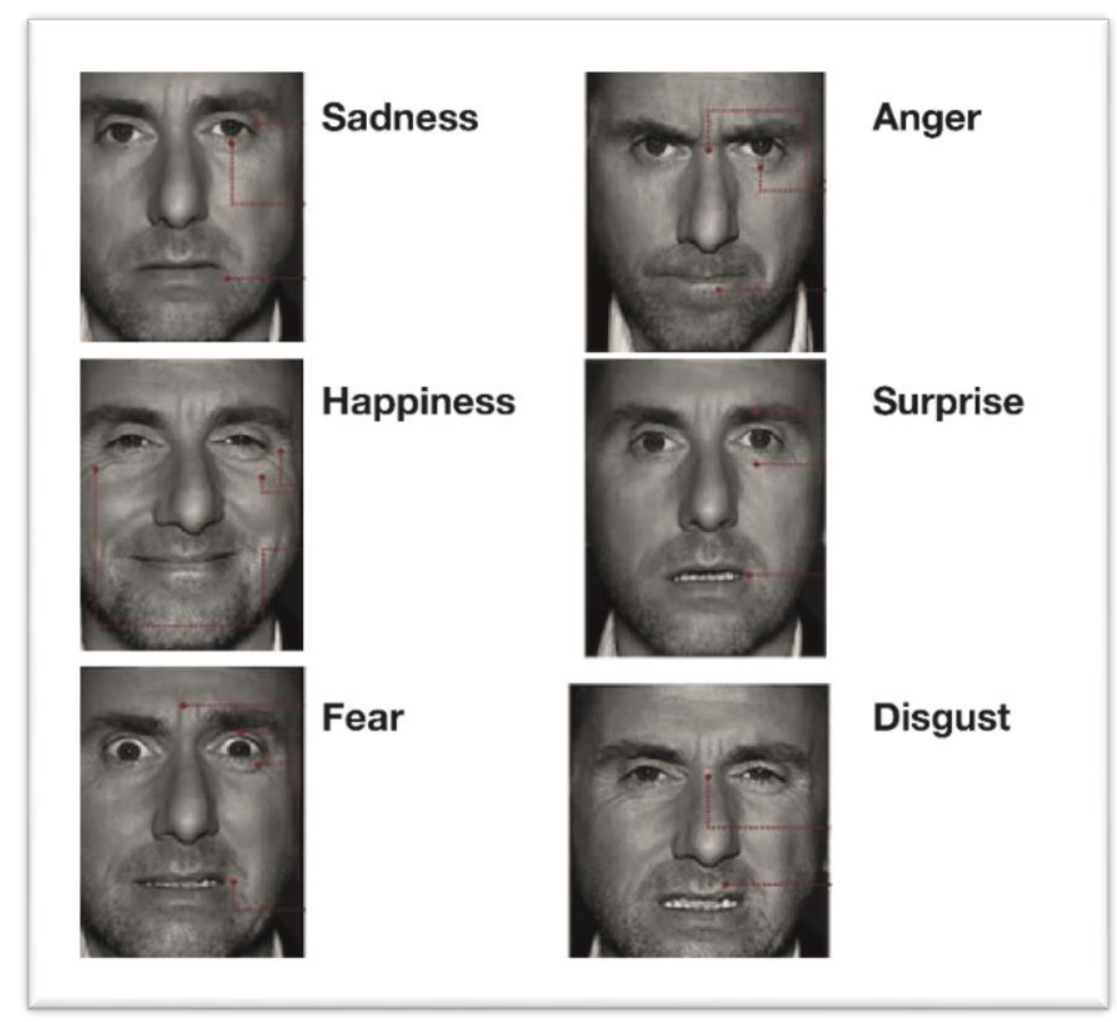

Figure 2.1 - The six basics emotions (Ekman, 1992)

\subsubsection{DIMENSIONAL THEORIES}

Dimensional theories try to explain emotions in terms of two or three dimensions. The most frequent dimensional characterization of emotions uses two dimensions: arousal and valence. Valence is related to a positive or negative evaluation and is associated with the feeling state of pleasure (vs displeasure). Arousal reflects the general degree of intensity felt. Low arousal is associated with less energy and high arousal with more energy. However, using these twodimensional theories it is difficult to differentiate emotions that share the same values of valence and arousal, as anger and fear. For this reason, a third dimension is often added. 
One of the most known dimensional models in 2D is the Circumflex Model of affect, proposed by Russell (Russell, 1980). Followers of this affective model suggest that each affective experience is a consequence of a linear combination of two dimensions, resulting in a particular emotion presented in Figure 2.2.

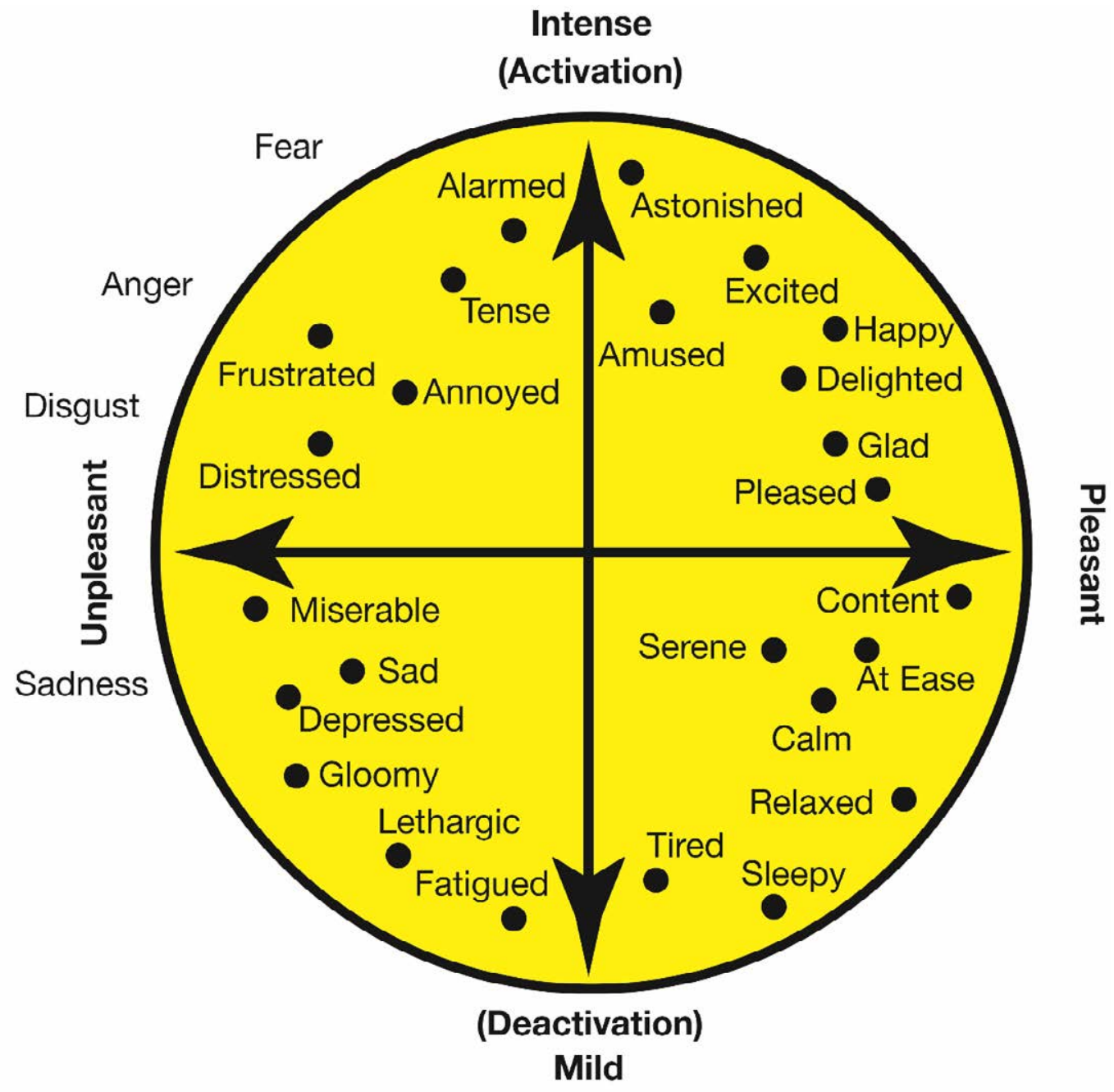

Figure 2.2 - The circumflex model of affect (Russell, 1980)

The Circumplex Model of Affect proposed by Russell (Russell, 1980) consists of the distribution of emotions in a system of coordinates. In this system of coordinates, the $x$-axis measures the valence, from positive to negative emotions, and the y-axis measures the level of arousal. The affective model falls to in a circle where it is possible to find emotions like pleasure $\left(0^{\circ}\right)$, arousal or activation $\left(90^{\circ}\right)$, displeasure or unpleasant $\left(180^{\circ}\right)$, and sleepiness or deactivation $\left(270^{\circ}\right)$. Fear, for example, is conceptualized as a neurophysiological state involving the combination 
of negative valence and increased activation in the central nervous system. In his study, Russell (Russell, 1980) presented a list of 28 words that people use to describe sentiment, moods, and emotions.

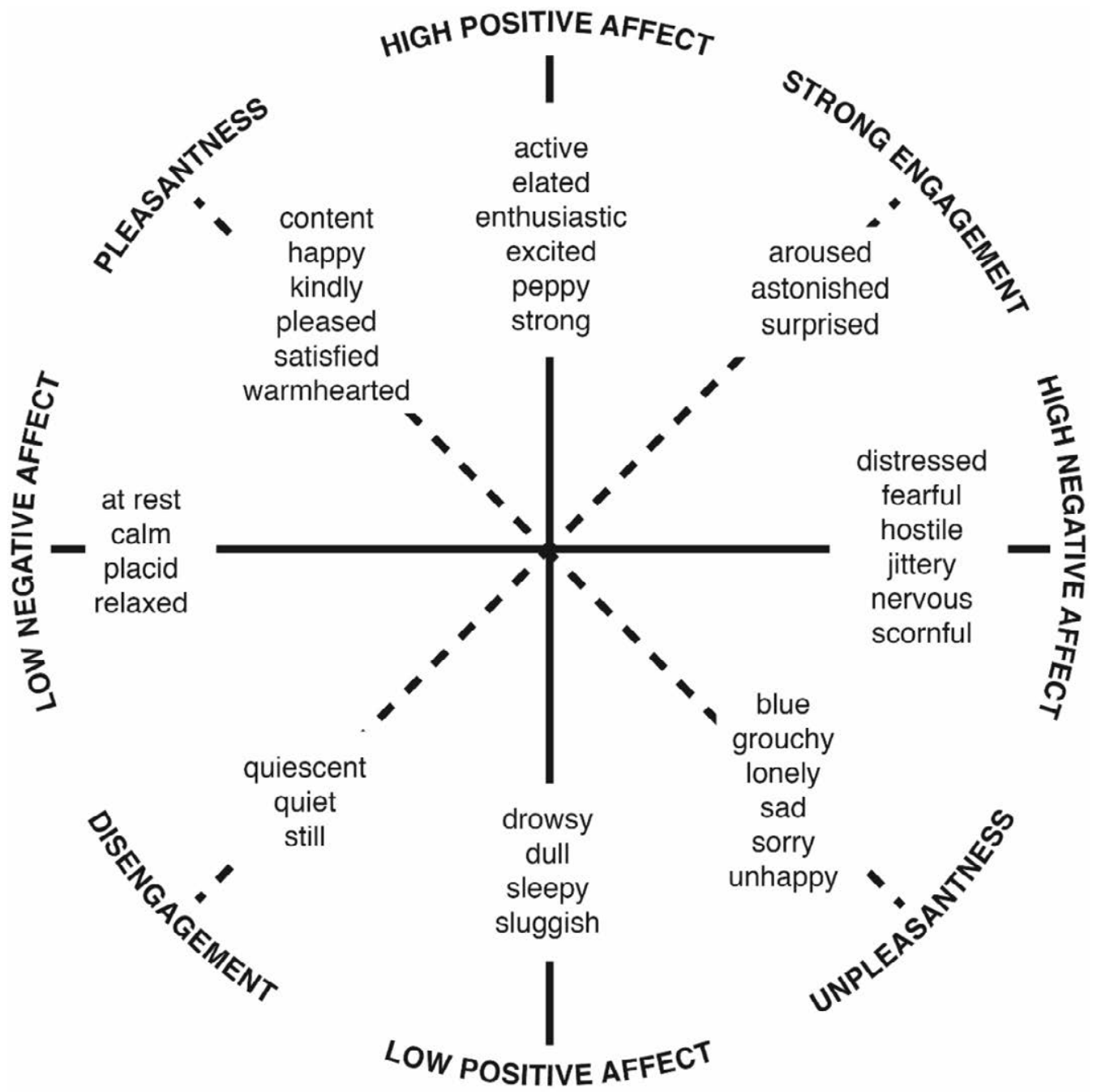

Figure 2.3 - Watson's two-dimensional structure of affect (Watson, Wiese, Vaidya, \& Tellegen, 1999)

Later, Watson, Wiese, Vaidya and Tellegen (Watson, Wiese, Vaidya, \& Tellegen, 1999) refined this model, which is presented in Figure 2.3. The proximity or the distance between the emotions represented in the circumference presupposes the similarity or the difference between the emotions. This model defines that emotions are less positively related when they are spaced approximately 90 degrees apart. At 90 degrees of separation, two affective states must be little or nothing related. In turn, at 180 degrees of separation, affective states must be negatively related. 


\subsubsection{APPRAISAL THEORIES}

As Leventhal and Scherer (Leventhal \& Scherer, 1987) "the third view emphasizes the distinct component of emotions, and is often termed the componential view". Emotional cognitive psychologists focus their studies mainly on the appraisal process. According to Dalgleish and Power (Dalgleish \& Power, 1999), the central idea is that emotions are triggered and differentiated by a subjective analysis of an event, situation or object. This cognitive assessment performed personally is called appraisal. So, emotion and reason are not disconnected. In fact, emotions require cognitive processes to generate or retrieve preferences and meanings. Emotions are triggered by personal interpretation of the annoying or cheerful aspects of an event, the appraisal. And the appraisal is a cognitive process, which triggers the emotions.

In that sense, emotion refers to relations among external incentives, thoughts, and changes in internal feelings. Occasionally, a unique combination of these features creates intense emotions of fear, joy, excitement, disgust, or anger (Picard, Papert, Bender, \& Blumberg, 2004). With emotions it is possible to build sensors for measuring the physical state and build successful algorithms for combining patterns of such measures and thus recognize the emotional states.

Based in appraisal theories concepts, the OCC Model (Ortony, Clore, \& Collins, 1990) is an interesting model of emotions that provides a structure of the emotion-eliciting conditions' and the variables that affect their intensities.

The OCC Model has recognized itself as the standard model for emotion synthesis, and it is often used to model users' emotional states. The model specifies twenty-two emotion categories based on the reactions of a subject to an event, the reactions of the subject to an action of a responsible agent, and the reactions of the subject that approaches an attractive or unattractive object.

The listed below and kept in the English language are: "happy for", "resentment", "gloating", "pity", "joy", "distress" "pride", "shame", "admiration", "reproach", "love", "hate", "hope", "fear", "satisfaction", "fears-confirmed", "relief", "disappointment", "gratification", "remorse", "gratitude" and "anger" (Ortony, Clore, \& Collins, 1990). The OCC model is shown in Figure 2.4. 


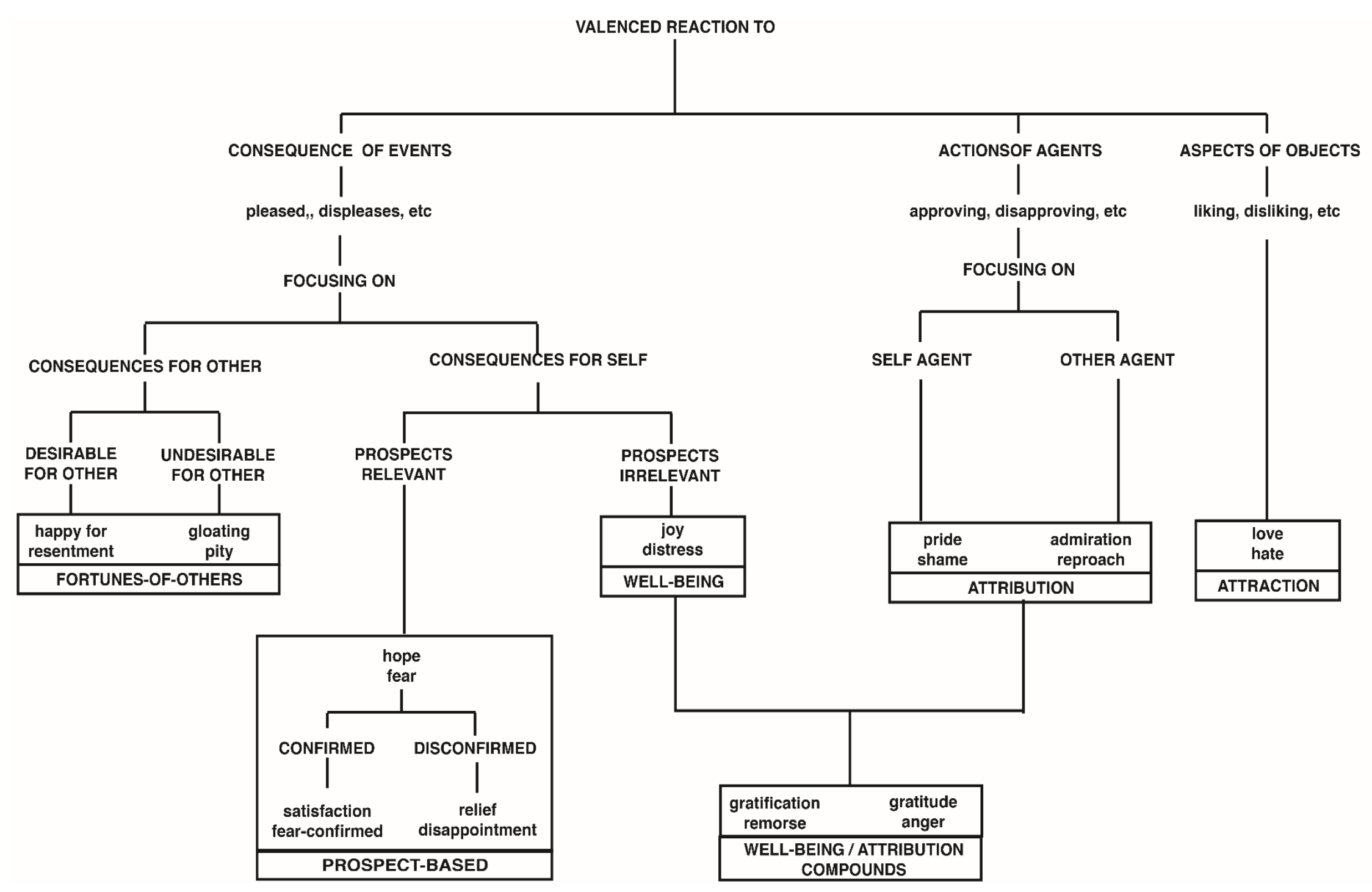

Figure 2.4 - OCC Model (Ortony, Clore, \& Collins, 1990) 
Based on the cognitive theory of emotions, the OCC Model considers that emotion arises from a cognitive evaluation, called "appraisal", generated by a person and based on the three aspects of the world: events, agents, and objects. Events are the sense, the occurrences by which people perceive what happens around them and their perception of the world in face of consequences. These emotions depend on the interest of an unconfirmed or confirmed event producing emotions like joy, distress, hope, fear, satisfaction, disappointment, relief, fears-confirmed, happy-for, resentment, gloating, and pity. Agents can be people, animals or, in some cases, other objects, or abstracted objects like institutions. These emotions depend on the value of the agent action as the result of an affected standard. The set of emotions include pride, shame, admiration, and reproach. And finally, objects, which are perceived by people as objects, present in the world, exerting an attraction, generating a positive or negative sentiment. These emotions depend on the interest of an object producing emotions like love or hate.

According to the appraisal cognitive structure, there are three interconnected components that underlie the perceptions of good or bad: goals, standards, and attitudes. The variable associated with reactions to events is desirability, that is, whether they promote or prevent someone from achieving their goals. The actions of an agent are analysed according to their compliance with standards and norms. And, finally, objects are analysed as attractive according to the compatibility of their attributes as to the preference of someone (Jaques \& Vicari, 2005).

Briefly, according to the OCC Model, emotions can arise from:

1. Analysing the consequences of events according to their desirability in relation to a person's goals;

2. Analysing (approving or disapproving) the actions of a person according to an individual's moral standards;

3. Analysing the attractive appeal of objects aspects.

The emotions of joy, sadness, fear, hope, satisfaction, relief, frustration and fear-confirmed arise by assessing the desirability of the consequences of an event for oneself. 
In the context of the interaction with pedagogical agents designed to improve the effectiveness of computer-based educational games, a model was proposed by Conati (Conati, 2002) called Dynamic Decision Networks (DDN). The model is based on the OCC cognitive theory of emotions but relies on deterministic rules to model the appraisal process. It is used to enable pedagogical agents for educational games to generate interactions custom made to the user's learning.

\subsection{RECOGNIZING EMOTIONS}

To recognize emotions, one of the purposes of $A C$ is to get that information through the human body. First, AC must be able to recognize the physical aspects of the human body, such as facial expression, voice intonation, and gestures or movements. AC also aims at recognizing physiological aspects such as breathing, skin colour, temperature, heart rate, blood pressure, and pupillary dilation.

\subsubsection{FACIAL EMOTIONS}

A facial expression is the result of the movements or positions of face muscles. Facial Expression Recognition (FER) plays an important role in natural human-machine communication (Chibelushi \& Bourel, 2003).

Most of FER research was influenced by the theories described in section 2.1.1 based on the work of Ekman (Ekman, 1992). Nowadays, advances in facial recognition software make it possible to recognize basic facial expressions such as anger, disgust, fear, happiness, sadness, and surprise.

Emotion recognition has been explored through three main types of databases: represented emotions, natural spontaneous emotions, and provoked emotions. The best results were obtained with the databases of provoked emotions since they obtain strong emotional expressions (Alepis, Stathopoulou, Virvou, Tsihrintzis, \& Kabassi, 2010).

\subsubsection{EYE TRACKING}

The several areas where the eye tracking process can be applied are neuroscience, psychology, computer science, industrial engineering, and marketing (Duchowski, 2002). 
Eye tracking is the process of measuring the motion of an eye in relation to the head position or the point of looking for (what one is looking at). Emotion is measured through the eye tracking hardware and a statistical program, which determines the excitement level when a visual image is presented (De Lemos, Reza Sadeghnia, \& Ólafsdóttir, 2008). Parameters analysis include pupil size, blink properties, and gaze. The pupil size is related to an emotional reaction, which indicates if there is an alteration in pupil size when a subject faces a positive or negative stimulus (Partala \& Surakka, 2003).

\subsubsection{BODY LANGUAGE}

Recognizing emotion through analysis of body language and posture appears to be an overlooked area in detriment of facial and speech in AC literature (Calvo, Member, \& Mello, 2010). There are a few studies indicating the advantages of recognizing body language (Bull, 1987). Tekscan's Body Posture Measurement System (BPMS) was one of the first systems created that used automated posture analyses to determine a student affective state in a learning environment (Mota \& Picard, 2003).

The BPMS system consists of two pressure sensors mounted on the chair. From those sensors the student postures are extrapolated in real time. This system recognizes postures related to affective states linked to a child's interest level while carrying out a learning task on a computer. The system has a neural network to classify the postures with an accuracy of $87.6 \%$. A few years later this model was improved by D'Mello and Graesser (D'Mello \& Graesser, 2009), who created a system that can detect learners' affect (boredom, confusion, delight, flow, and frustration) by monitoring their gross body language (Calvo, Member, \& Mello, 2010). The accuracy of this system is between $73 \%$ and $83 \%$ (Mota \& Picard, 2003).

\subsubsection{EMOTIONAL SPEECH RECOGNITION}

The vocal aspect of a communication also carries information about speech emotional contents of a person and speech recognition consists on the ability of a machine or program to identify words or phrases from the spoken language. The speech recognition can be divided into two parts: an explicit message, consisting of 
what was said; and an implicit emotional expression, involving how the message was said.

The main application of speech recognition resides in assisted technology to help people with disabilities. So, Speech Emotion Recognition (SER) aims to recognize the user's emotional state in his speech signal (Wu, Falk, \& Chan, 2011).

The major conclusion of SER relies on the possibility to recognize emotion from a speech. Although, there is some difficulty in recognizing certain kinds of emotions like disgust, there are others that can be recognized with more accuracy like sadness and fear. Also, the pitch of a speech seems to be connected to the level of arousal. In addition, affect discovery in speech has accuracy rates lower than a facial expression for recognizing basic expressions.

\subsubsection{EMOTION DETECTION IN TEXT}

Emotion detection in a written text consists in to determine the emotional or attitude context within the written language or transcripts of oral communication.

There are three approaches of emotion detection in the text:

1. The first approach aimed to understand how the text could express an emotion or how the text could generate different emotions. These studies began by finding a resemblance in how people of different cultures communicate (Osgood, May, \& Miron, 1975). These authors used a Multidimensional Scaling (MDS) procedure to create models of affective words based on words evaluations provided by diverse cultures. The dimensions considered in these studies were: evaluation, potency, and activity. Evaluation refers to the quantification of a word in relation to an event it portrays and if that event is pleasant or unpleasant. Potency refers to how the word is related to a level of intensity, strong words as opposed to weak words. Activity refers to a word as active or passive (Calvo, Member, \& Mello, 2010).

2. The second approach includes a lexical analysis of the text, as a result the affective state can be inferred (Cohn, Mehl, \& Pennebaker, 2014). Most of these studies use the Linguistic Inquiry and Word Count (LIWC) (Pennebaker, Booth, \& Francis, 2007), which is text analysis software that 
determines if the text has positive or negative emotions, self-references, causal words, in over 70 language dimensions.

3. The third approach accepts that people sharing the same language have comparable notions for different emotions. Based on this, a lexical database was built containing emotional terms.

Affective Norms for English Words (ANEW) (Bradley \& Lang, 1999) was developed to offer a set of normative emotional ratings for a large number of words in the English language. The objective is to construct a series of verbal materials that were rated in terms of pleasure, arousal, and dominance. ANEW complements the International Affective Picture System (IAPS) (Lang, Bradley, \& Cuthbert, 1997) and the International Affective Digitized Sounds (IADS), which are collections of photographs and sounds stimuli, respectively, that also contain affective ratings.

Pang and Lee (Pang \& Lee, 2006) reviewed the concept of sentiment and opinion analysis, which refers to the application of natural language processing, computational linguistics, and text analytics to recognize subjective information, like emotion, in the text.

\subsubsection{OTHER EMOTION RECOGNITION}

There are some other approaches to obtain the emotion recognition like physiology, brain imaging techniques, and multimodality of emotions recognition.

With the approach of physiology, affection recognition occurs by identifying patterns in the physiological activity. This approach was inspired by the areas like psychology and psychophysiology and tries to understand what physiological patterns are behind them and what the implications in behavior are. To monitor these physiological patterns, the electrical activity produced by the brain, heart, eyes, skin, and muscles is recorded. The methods of recording include: Electroencephalography $(E E G)$, that measures brain activity; Electrocardiogram (ECG), that measures heart activity; Electrooculogram (EOG), that measures eye movement; Electro Dermal Activity (EDA), that measures electrical conductivity of the surface of skin; and Electromyograms (EMG), that measures muscles activity (Calvo, Member, \& Mello, 2010). 
The approach of brain imaging techniques is used in the fields of affective neuroscience, which tries to map the neural circuitry that occurs during an emotional experience (Dalgleish, Dunn, \& Mobbs, 2009) (Paradiso, 2002).

In this approach, the techniques used by neuroscientists include fMRI, EEG, among others. $\mathrm{FMRI}$ is based on the MRI technology and it is a non-invasive test that uses a strong magnetic field and radio waves to create detailed body images. Therefore fMRI monitors the blood flow in the brain to detect activity areas and provides a map of which parts of the brain are active during an emotion or feeling. EEG technique records brain spontaneous electrical activity through mapping. Due to the absence of an emotion neural model, this method is limited and frequently supported by other techniques like the fMRI. One of the measures taken with EEG is the Event-Related Potential (ERP). ERP is a measured brain response that is directly the result of a thought or a perception. It basically measures any electrophysiological response to an internal or external stimulus (Olofsson, Nordin, Sequeira, \& Polich, 2008).

Finally, the multimodality of emotion recognition approach refers that emotional response has multiple manifestations both physical and behavioral. This approach based on the techniques of Multimodal Human-Computer Interaction $(\mathrm{MHCl}) . \mathrm{MHCl}$ relies on several research areas such as computer vision, artificial intelligence, psychology, and others. The study of $\mathrm{MHCl}$ entails the knowledge of three variables: user, system, and the interaction between them (Jaimes \& Sebe, 2007). This technique wants to interact with: visual interaction (facial expression, head pose, gesture, body movement, and postures), auditory (pitch, loudness, speaking rate), tactile (heart rate, skin conductivity), and brain signals (EEG).

\subsection{AfFECTIVE States}

According to Khurana (Khurana, 2007) emotions have two components: the mental component (cognitive) and the physical component (body). In line with Shaver, Schwartz, Kirson and O'Connor (Shaver, Schwartz, Kirson, \& O'Connor, 1987 ) emotion can be classified into three categories: primary, secondary, and tertiary emotions. Primary emotions occur as a response to some kind of event, which can cause a detectable physical response and trigger emotions such as fear, 
joy, love, sadness, surprise, anger. From these emotions can result the other subcategories (secondary and tertiary). Table 2.1 is presented a summary of these types of emotions.

All the affective states explained next occur by assessing the event desirability by itself:

- Joy (happiness) can arise due to the occurrence of some desired event.

- Sadness occurs by the existence of an undesirable fact or event.

- Satisfaction happens when an expected event that has already occurred is desirable.

- Frustration happens when there is confirmation that the expected and desirable event did not happen.

- Hope can arise when there is no confirmation about the event happened, ie when the individual is still experiencing expectation.

- Fear occurs when the consequences of the event are undesirable according to the person's goals, and hope, otherwise.

- Fear-confirmed happens when an expected event that has already occurred is undesirable.

- Relief happens when there is confirmation that the expected and not desirable event did not happen.

Each event causes different emotions in people. For example, an event can make a person feel happy and this same event, in another person or in another situation can cause sadness. In addition, the person who felt happy before an event may feel pity for another person, seeing their reaction to the event.

Once the object of evaluation is the action of a person, pride, shame, admiration or disapproval may occur. Pride and shame happen when the individual approves (or disapproves) of his/her own action. When another person's action is approved (or disapproved) admiration or disapproval can be experienced.

If the individual analyses his/her own actions, he/she can feel gratification when he/she approves of his/her action and he/she has positive consequences for himself/herself, and remorse when he/she disapproves of his/her action and it has negative consequences. While a person evaluates another person's actions, he/she 
may feel gratitude for that person when he/she approves that other person's actions, and also when the resulting event has positive consequences for him or her. Likewise, anger will arise when it disapproves of another's action and it still has negative consequences for each one of us.

Table 2.1. Emotions (Shaver, Schwartz, Kirson, \& O’Connor, 1987).

\begin{tabular}{|c|c|c|}
\hline $\begin{array}{l}\text { Primary } \\
\text { Emotions }\end{array}$ & $\begin{array}{l}\text { Secondary } \\
\text { Emotions }\end{array}$ & Tertiary Emotions \\
\hline \multirow[t]{2}{*}{ Fear } & Nervousness & $\begin{array}{l}\text { Anxiety, Apprehension, Distress, Dread, Tenseness, Uneasiness, } \\
\text { Worry }\end{array}$ \\
\hline & Horror & Alarm, Fright, Hysteria, Mortification, Panic, Shock, Terror \\
\hline \multirow{2}{*}{ Joy } & Cheerfulness & $\begin{array}{l}\text { Amusement, Ecstasy, Gaiety, Euphoria, Bliss, Elation, Delight, } \\
\text { Happiness, Jubilation }\end{array}$ \\
\hline & Zest & $\begin{array}{l}\text { Enthusiasm, Excitement, Exhilaration, Thrill, Contentment, Relief, } \\
\text { Optimism, Pride, Enthrallment }\end{array}$ \\
\hline \multirow{3}{*}{ Love } & Affection & Fondness, Attraction, Adoration, Sentimentality, Caring \\
\hline & Lost & Arousal, Desire, Passion, Infatuation, Obsession \\
\hline & Longing & Longing \\
\hline \multirow{6}{*}{ Sadness } & Suffering & Agony, Hurt, Anguish \\
\hline & Disappointment & Dismay and Displeasure \\
\hline & Shame & Guilt, Remorse, and Regret \\
\hline & Neglect & Insecurity, Alienation, Homesickness, Embarrassment, Humiliation \\
\hline & Sadness & Depression, Unhappiness, Misery, Melancholy, Gloom, Despair \\
\hline & Sympathy & Pity, Sympathy \\
\hline Surprise & Surprise & Astonishment, Amazement \\
\hline \multirow{6}{*}{ Anger } & Rage & $\begin{array}{l}\text { Fury, Wrath, Bitterness, Loathing, Resentment, Hate, Loathing, } \\
\text { Frustration and Exasperation }\end{array}$ \\
\hline & Irritation & Agitation, Aggravation, Grouchiness \\
\hline & Disgust & Revulsion, Contempt, Jealousy, and Torment \\
\hline & Exasperation & Exasperation, Frustration \\
\hline & Envy & Envy, Jealousy \\
\hline & Torment & Torment \\
\hline
\end{tabular}

Finally, an individual may or may not like aspects of an object. When the object exerts a positive attraction it feels affection to the object, otherwise, it feels aversion. 


\subsection{AFFECTIVE LEARNING}

Affective Learning according to recent definitions involves the combining of thinking and feeling in how people learn. The goal is better understood when it uses knowledge and skills. For that, it is important to create applications played by mental dispositions in how a person views, engages, and values learning (Stricker, 2009).

According to the literature, there is a connection between emotion and the learning process (McDaniel, et al., 2007) (Regan, 2003). Although, this process is not simple or direct, it is accepted that positive and negative emotional states can cause different kinds of thinking and can have an effect on the learning perspective.

There are several types of learning. In 1956 Benjamin Bloom (Best, Floyd, \& McNamara, 2008), identified three domains of educational activities cognitive: mental skills (Knowledge); affective: growth in feelings or emotional areas (Attitude); psychomotor: physical or manual skills (Skills). The combination of all domains influences the way one learns and the way rational decisions are made.

Some research indicated that a slight positive mood can produce an effect on memory, well-organized open-minded, flexible problem-solving and thinking as well as more efficiency and thoroughness in decision-making. This can be found in groups of different ages and professions (Picard, Papert, Bender, \& Blumberg, 2004), (Isen, 2001). The effect on cognition is not restricted to positive states of mind. Negative affective states like anger, sadness or fear can influence the brain activity affecting the thought process (Isen, 2001).

One of the challenges that affective learning faces are to bringing together the theorists and practitioners from different fields in order to refine the language used in relation to affect and learning (Picard, Papert, Bender, \& Blumberg, 2004). It is necessary to create a model that can provide effective results in the learning process. The model built has to take into account other factors like the model or profile of the user and also the learning style.

\subsection{USER'S BEHAVIOR}

A very interesting quote from Johann Wolfgang von Goethe, the worldfamous German novelist, reads: "Behaviour is the mirror in which everyone shows 
their image." Hence, task execution is not done similarly by all users. Each person has his/her own behavior. This behavior can be derived from several factors, such as the biological characteristics of the user; characteristics of the task, and environmental factors.

The recognition of these behaviors is already used in new technologies as referred in subsection 2.2. For example, the recognition of users as a form of authentication on a device or software can be done by tracking behavioral biometrics, such as fingerprints, face recognition or iris recognition (Benevenuto, Rodrigues, Meeyoung, \& Almeida, 2009). This behavioral biometrics can be classified into five categories based on information type (Yampolskiy \& Govindaraju, 2008):

$>$ Behavioral Biometric based on the analysis of text extracts or drawings produced by the user;

> Behavioral Biometric based on computer interaction;

- Device usage, such as a mouse and keyboard. Devices that can capture the muscles' actions;

- Software interaction: strategy, and knowledge;

$>$ Behavioral Biometric based on monitoring data coming from a low-level action in software, such as access log, storage activity, and calls systems;

> Behavioral Biometric based on data from motor skills;

As an example to recognize the lack of attention on user's behavior, different ways of capture and classify attention were discovered. One of the first ways to try to quantify the attention level was a questionnaire. This questionnaire is presented to the user. After the user answers to the questionnaire, the study's author analyses the answers and presents the conclusions. The advantage of the questionnaire is that they are an inexpensive approach to collect vast amounts of information, they do not represent a very significant effort for the researcher, it is easy to compile data, and can be administered either by the researcher or by anyone else, possibly remotely. The disadvantage of this approach is that is more qualitative and depends on the author's interpretation (Mancas M. , 2015).

The more quantitative approach is the one that uses biometrics behavioral. It is an approach more focused on the perception stimuli. Eye-tracking is one of the 
most used techniques. Using this technology when performing a task on a computer, it is possible to know the screen area where the eyes are directed and, consequently, where the focus of attention is. So, it is possible to conclude if the user was with the visual attention directed to the screen area where the task is positioned (Mancas M. , 2015) , (Duc, Bays, \& Husain, 2008). This is, however, a very intrusive methodology which raises privacy concerns, especially in the workplace.

Another approach is based on neural activity. The most used technique is EEG, which uses electrodes placed on the scalp that read the frequency of brain waves and, with the acquired data, analyses the brain activity during a task. In many studies, the most important component is MMN (mismatch negativity). This component is the indicator of a brain reaction to a pre-attention process. Other techniques used are functional imaging, fMRI, MEG, functional imaging, and PET scan (Mancas M. , 2015).

Regarding techniques that measure attention, the first two techniques described are intrusive approaches. In the case of brain activity measures, it is necessary to place some devices on the user's body to capture the data. This can interfere with the performance of the user and cause stress as it is also intrusive. Regarding eye tracking, and although it does not require the use of devices placed on the body, the technique is an approach that has raised issues related to privacy and data protection (especially with underage individuals), which makes it unsuitable for academic environments.

\subsubsection{PATTERN INTERACTIONS USING BEHAVIOR BIOMETRICS}

Another method to measure attention is through the use of keyboard and mouse interactions, which might provide a non-obtrusive and easy-to-use method of continuous monitoring.

The user keystroke and mouse behaviour might change in response to several factors like stress and health status (Monrose \& Rubin, 2000), (Araújo, Sucupira, Lizarraga, Ling, \& Yabu-Uti, 2005). Based on keyboard typing patterns and mouse movement's pattern, researchers also observed a certain amount of inherent variability in an individual's typing pattern (Araújo, Sucupira, Lizarraga, Ling, \& YabuUti, 2005). 
Figure 2.5, presented a behaviour biometrics devices. Mouse and keyboard tracking are techniques also used to measure and classify attention. These techniques have already been used to measure other variables such as stress (Carneiro, Novais, Pêgo, Sousa, \& Neves, 2015) and mental fatigue (Pimenta, Gonçalves, Carneiro, Riverola, \& Novais, 2015), (Pimenta, Carneiro, Neves, \& Novais, 2016).

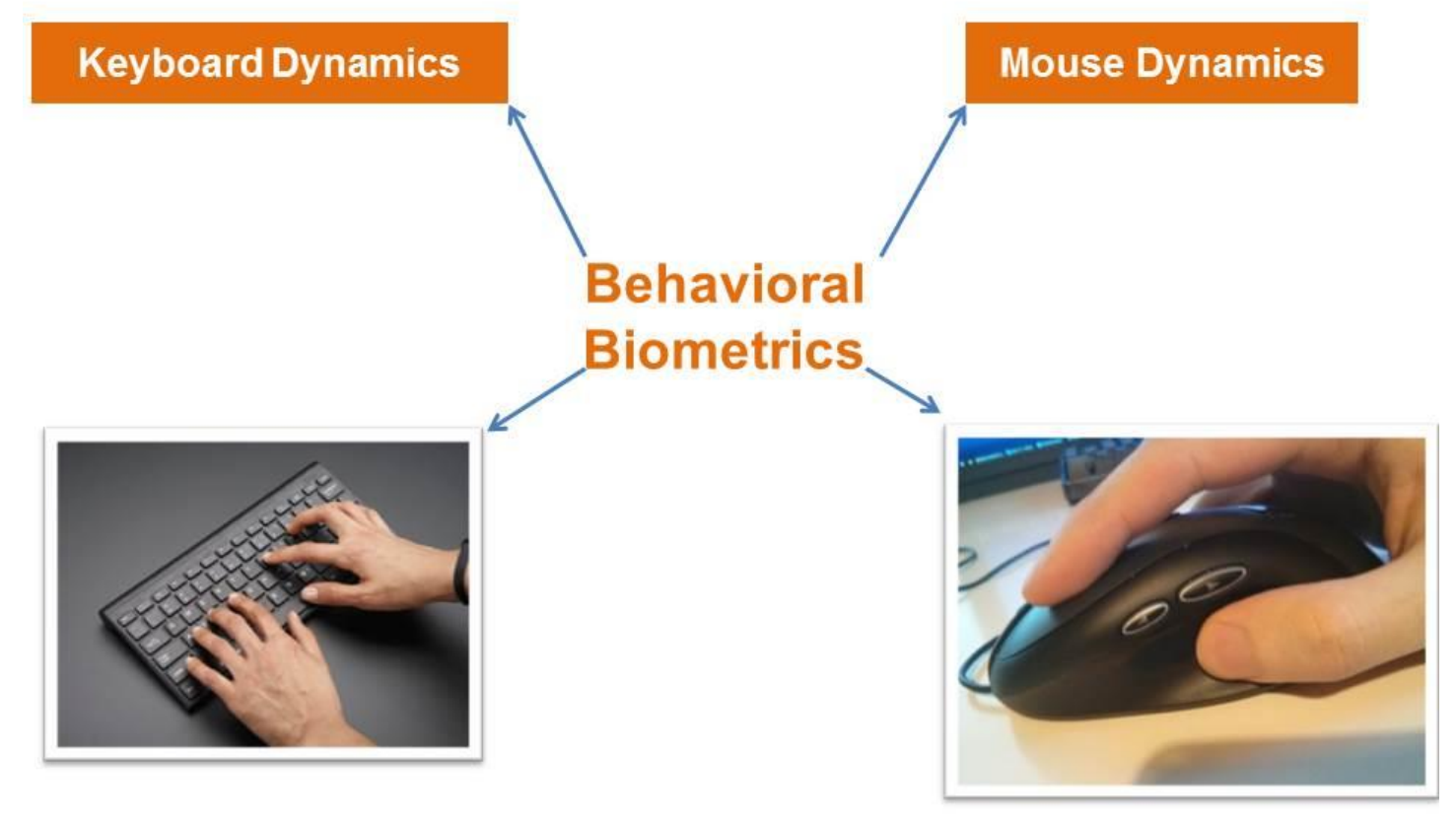

Figure 2.5 - Behavioral Biometrics devices.

In the case of the stress study (Carneiro, Novais, Pêgo, Sousa, \& Neves, 2015), the data was captured during an online exam of medical students. The captured data was related to mouse movement and keyboard usage. The mouse and keyboard tracking technique is a non-invasive approach because the data captured is compiled by a background software and the user does not have the perception that he/she is being monitored. This is an advantage over the two previous approaches as the user is not affected in any way by the collection of data.

The way user types may indicates his/her state of mind and attention level. Pressing keys hard and rapidly could indicate an altered state such as anger, while 
taking too much time could mean sadness. The same occurs with mouse movements. A similar system that monitors user's behaviour from standard input devices, like the keyboard or the mouse, is proposed by Zimmermann, Guttormsen and Danuser (Zimmermann, Guttormsen, Danuser, \& Gomez, 2003). Thus, keyboard and mouse dynamics can provide relevant behavioural information about the affective and cognitive state of the user.

Hernandez, Paredes and Roseway (Hernandez, Paredes, Roseway, \& Czerwinski, 2014) explored the possibility of using a pressure-sensitive keyboard and a capacitive mouse to discriminate between stressful and relaxed conditions in a laboratory experiment in which participants performed several tasks under stressed and relaxed conditions, during 30 minute sessions.

From the analysis of Human-Computer Interaction, it also found that people tend to interact differently with different applications and in different contexts (Carneiro, Saraiva, Martinho, Marreiros, \& Novais, 2018). For example, although both tasks involve typing, people tend to type differently if they are in a messaging application and in a word processing application (Pimenta, Carneiro, Neves, \& Novais, 2016).

\subsection{SUMMARY}

The AC is built over "affect model" based in the various sensors-captured information in order to perceive, recognize, and understand human's emotions and provide better feedback. The "affect model" used for AC is the OCC Model, which consider that emotion can arise from events, actions, and objects. The consequences of events cause different types of emotions, focusing on consequences for others or consequence for himself/herself. The actions of agents also cause different types of emotions focusing self-agent or another agent. Finally, the aspects of objects cause different types of emotions.

AC can be applied in different fields like $\mathrm{HCl}$; text-based communications, and virtual reality; monitors humans; and education systems. There are several subjects that study emotion and affects. The main research theories that analyse emotion are discrete, dimensional, and appraisal theories. 
The AC applications that can recognize emotions are facial emotions; eye tracking; body language; emotion speech recognition; emotion detect in the text; physiology; brain imaging techniques; and multimodality of emotions recognition.

There are several affective states depending on the model chosen. Furthermore, affective learning indicated that emotions influence the learning process, and it is accepted that positive and negative states obtain different learning outcomes (Carneiro, Durães, Bajo, \& Novais, 2016). It is important to be able to monitor user's behaviors and understand how these behaviors influence and interconnect with the user's attention level.

The mouse tracking and keyboard tracking techniques are a non-invasive approach because the data captured is made by a background software and the user does not have the perception that it is being monitored. This is an advantage over the other approaches because the users have no idea that they are being supervised and do not change their pattern behavior. This approach will be more detailed in section 4 because it was the approach used in this work.

In the next chapter, the state of art will be described as well as all the different views from different authors. All the definition of attention, engagement, learning, and learning styles will be presented. 
"Attention - The act of directing the mind to listen, see, or understand - Cambridge

Dictionary

\section{ATTENTION AS A FORM OF ENGAGEMENT}

According to the authors Mancas, Ferrera, Ritche and Taylor (Mancas, Ferrera, Ritche, \& Taylor, 2016) attention is the first action of perception that analyses the outer real world and turns it into an inner conscious representation and it is also the gate to conscious awareness.

The brain receives a huge amount of information from vision, audition, touch, smell or taste. This gigabit of sensorial data flows every second into the brain, which overloads the capacity to think and respond coherently. Attention is the key to survival and a sign of limited computation capabilities, because it is necessary to provide a brain or computer with the capacity of selecting relevant information and prioritizing tasks.

\subsection{AtTEntion CONCEPT}

Generally, there is no universally accepted definition of attention because there is a diversity of disciplines that are focused on it. In the past, only philosophy studied attention. However, in present days, attention is highly important for other fields like psychology, chemistry, anatomy, and even computational science (Mancas M. , 2007). 
The concept of attention has had different definitions since the nineteenth century, but none was considered as an universal definition. With the existence of these multiple sets of disciplines that study attention, its definition diverges depending on the field of study. For example, we can differentiate the concept of attention in human beings and machines. In humans' beings, attention is processed in the brain, while in machines there is a processor unit with a certain memory capacity that will process data. As with the brain, computers should analyze more and more data, but unlike the brain, they do not or do rarely "pay attention" (James, 1890) to the data. Figure 3.1. presents an evolution of areas that study the attention concept.

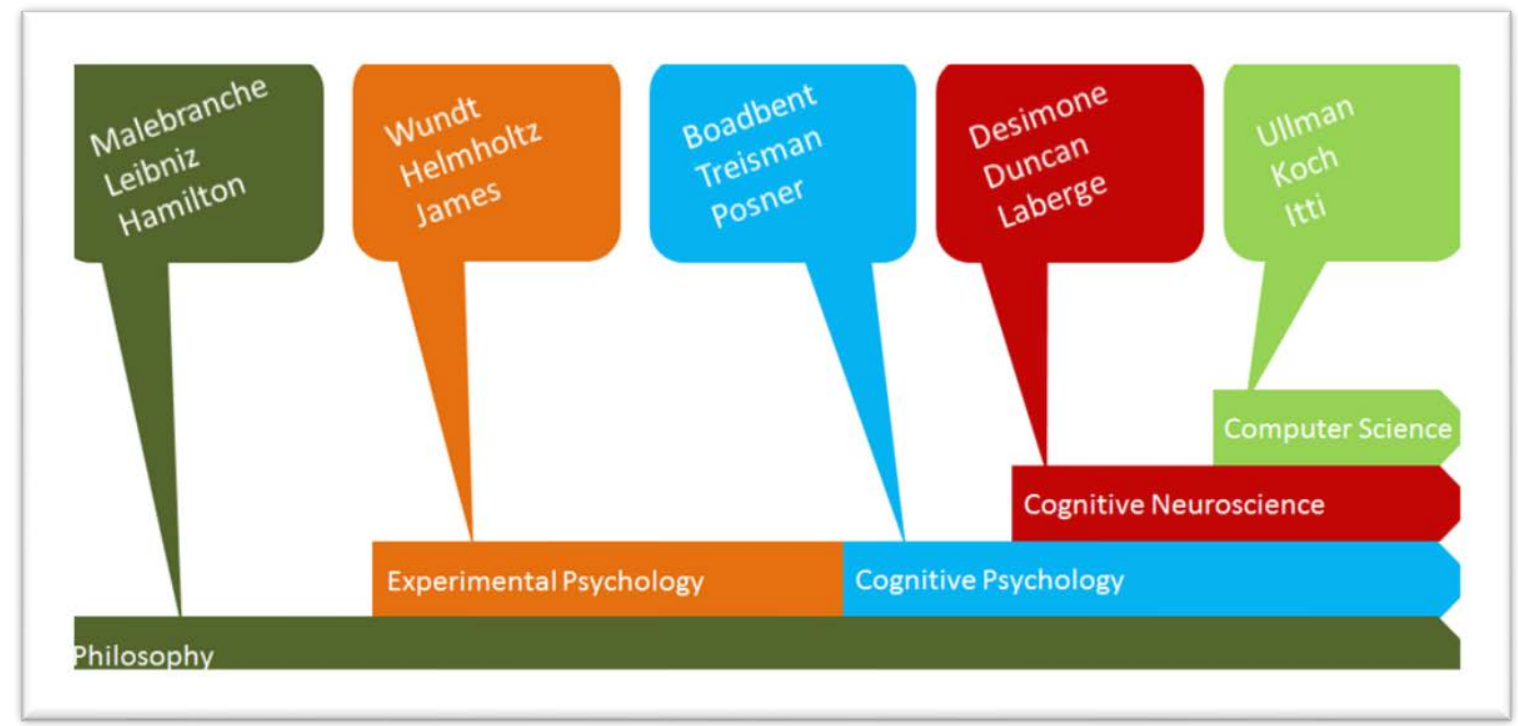

Figure 3.1 - Areas that study attention.

Attention is a very complicated process through which one individual is able to continuously analyze a spectrum of stimuli and, in a sufficiently short amount of time, selected one to focus on (Estes, 2014).

\subsubsection{ATTENTION CONCEPT IN THE FIELD OF PHILOSOPHY}

The field of Philosophy was the first subject to study attention in a theoretical form. In this field, instances of attention differ along several dimensions of variation, like a perceptual phenomenon, a phenomenon related to action, or voluntary. 
Attention is also a topic of philosophical interest because of its apparent relations to consciousness, yet there is controversy over whether this relation of consciousness is attention and how conscious experience can be modulated by attention (Mole, 2012).

It has began with the Greek philosophers who briefly treated selective attention. Descartes, in his book Meditations, briefly indicated the important role of attention to solve the apparent conflict between clear ideas and doubts. So he proposed that it is only when we pay attention to objections that clear and distinct ideas provide a place where doubt has no place (Mole, 2012).

The expression "human attention" was questioned by Nicolas Malebranche, who focused on the role of attention linked to consciousness. G. W. Leibniz in the eighteenth century introduced the concept of "apperception" and attention is seen as a reflexive and involuntary gate for consciousness (Mancas, Ferrera, Ritche, \& Taylor, 2016).

At this time, attention was understood as the focused in one task at a time, based on the nature of selectivity of our mental lives. This process is one of the principal points of disagreement between the existent theories of attention: for a first group of the most influential theories, selectivity of attention, it is the result from limitations in the brain's capacity to process the complex properties of multiple perceptual stimuli; for a second group, selectivity of attention, it is the result of limitations in the thinking subject's capacity to consciously entertain multiple trains of thought; finally, for a third group, selectivity attention does not refer to limitations in ability but relates it to the selectivity that is required to maintain a single coherent line of action, with the amount of sensory information received and the expected precision, or with the mutual competition between inhibitory processing flows (Mole, 2012).

In the nineteenth century, Sir W. Hamilton changed the previous perspective on attention. He introduced the idea of "divided attention", which mean that in some situations people can remember more than a fact that occurs at the same time (Mancas, Ferrera, Ritche, \& Taylor, 2016). 


\subsubsection{ATTENTION CONCEPT IN THE FIELD OF EXPERIMENTAL PSYCHOLOGY}

The study of attention by psychologists goes back to the beginnings of modern psychology. In the nineteenth century, W. Wundt introduces the concept of conciseness and attention in the field of psychology and initiates a series of studies on the speed of mental processing. He studies the time between the occurrence of the stimulus and the attention paid by a person (Mancas M. , 2015), (James, 1890).

$H$. Von Helmholtz in the second half of the nineteenth century, studies the movement of the eyes. For him, humans need to move their eyes around the whole visual field in order to distinct scenes, which called "overt attention". However, he also concludes that humans have the ability to focus on different parts of a scene without moving the eyes, which he called "covert attention" (Mancas M. , 2015), (James, 1890).

One of the most famous psychologists who dealt with the subject at that time was William James. According to his book, Principle of Psychology (James, 1890), the perception of the existence of selective attention was not easy because many psychologists of the time believed that everything that occurred in the human mind was derived from experiences. To William James, the breakthrough in his line of thought was when he was placed in the presence of several objects and only a few caught his attention. He concluded then that what makes the human being experience this stimulus is the interest that they cause in a person.

His (James, 1890) definition of attention is:

"Everyone knows what attention is. It is the taking possession by the mind in clear and vivid form, of one out of what seem several simultaneously possible objects or trains of thought. It implies withdrawal from some things in order to deal effectively with others, and is a condition which has a real opposite in the confused, stunned, scatter brained state."

It also says that the essence of attention is the focus, concentration, and awareness (Mancas M. , 2007). 


\subsubsection{ATtENTION CONCEPT IN THE FIELD OF COGNITIVE PSYCHOLOGY}

In the second part of the twentieth century, Broadbent proposed a model in which he described the selective properties of attention. He referred that attention acts like a filter of important information based on basic physical characteristics.

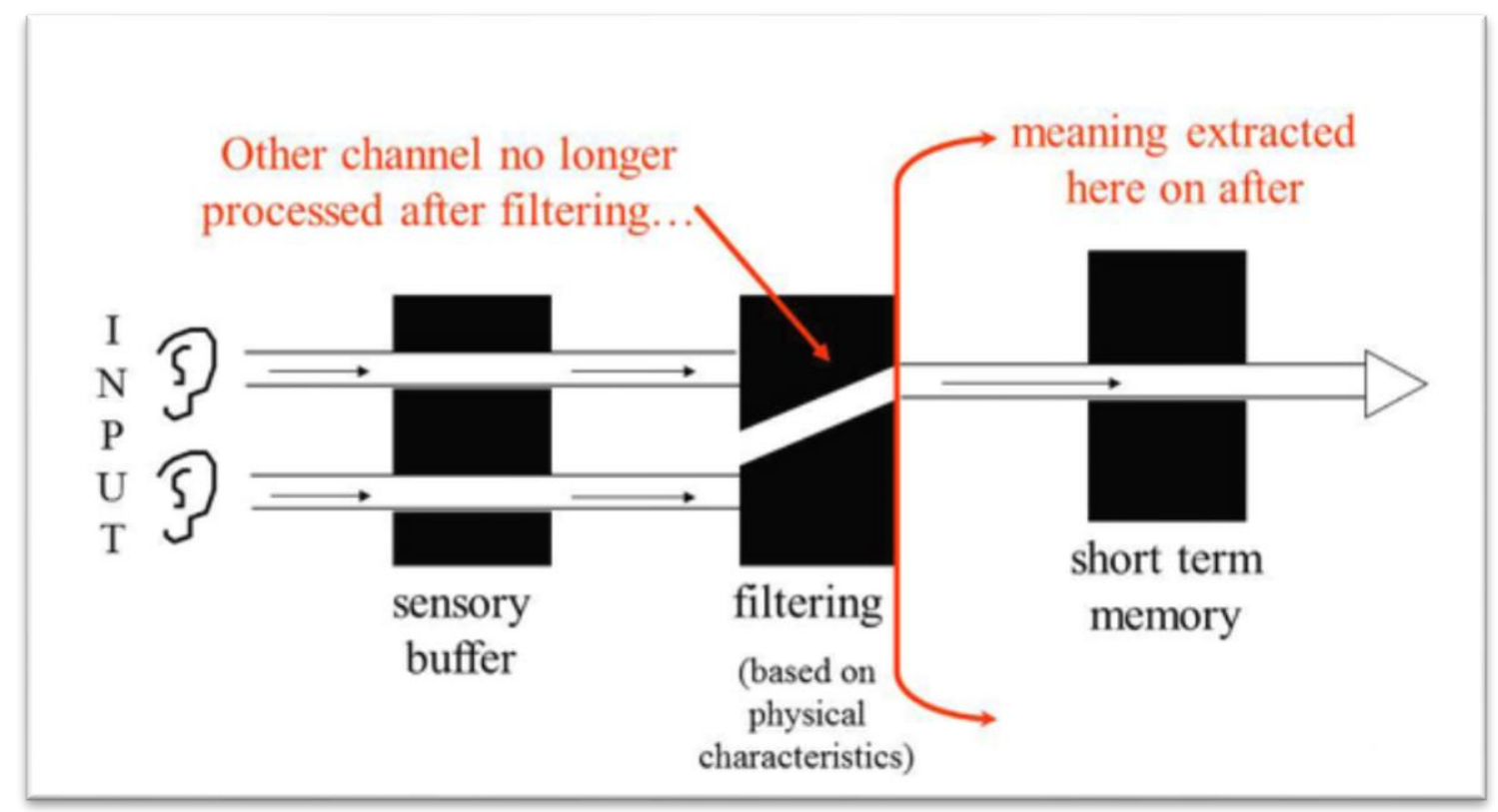

Figure 3.2 - BroadBent's model (McLeod, 2008)

In Figure 3.2 at the same time, two inputs are received and are allocated in the sensory buffer. In the sensory buffer, the new information is preserved, for a small period of time, to be processed or forgotten. An initial input is chosen based on its basic features and passes through the Broadbent's filter. Subsequently, the new information is saved in the short-term memory. The initial information is maintained in the sensory buffer while waiting to be processed or forgotten (Estes, 2014), (Mancas M. , 2015).

Another theory was the filter models proposed by Treisman. The Attenuated filter is a soft version of the Broadbent's model. The crucial difference is that the Treisman's filter attenuates instead of eliminating the ignored material (Estes, 2014), (Mancas M. , 2015).

In the eighties, other theories emerged with $\mathrm{M}$. Posner, who proposed a theory of attention based on target detection, visual orienting, and alertness (Mancas M. , 2015). 


\subsubsection{ATTENTION CONCEPT IN THE FIELD OF COGNITIVE NEUROSCIENCE}

Cognitive neuroscience involves techniques like EEG, eye-tracking devices, and tools that provides new insights into brain-behavior. In order to obtain the new insights on brain-behavior the following methods were used (Mancas, Ferrera, Ritche, \& Taylor, 2016):

$>$ Psychophysiological methods - scalp recording of EEG that measures the large-scale electric activity of the neuro and MEG, which measures the magnetic fields produced by electrical currents in the brain.

$>$ Neuroimaging methods - functional MRI and PET scan images that scan areas which have intense activity. Magnetic resonance spectroscopy can provide information about specific neurotransmitters.

$>$ Electrophysiological methods - single-cell recordings that measure the electrophysiological responses of a single neuron using a microelectrode system.

> TMS and TDCS methods - can be used to stimulate a region of the brain and to measure the activity of specific brain circuits in humans.

$>$ Multielectrode technology methods - allows the study of the activity of many neurons simultaneously showing how different neuron populations interact and collaborate.

Applying those methods two main families of theories have been constructed Desimone - Duncan, and Laberge.

Desimone \& Duncan Model also called as Biased Competition Model, whose main idea is that at any moment there is more information in the environment that can be processed and relevant information always competes with irrelevant information to influence behavior. So the model increasing the influence of behaviorally relevant information and decreasing the influence of irrelevant information based on selective area activity of the brain. To them, attention is "an emergent property of many neural mechanisms working to resolve competition for visual processing and control of behavior" (Desimone \& Duncan, 1995). 
Laberge Model is based on neuropsychological and neuroimaging studies, which concludes that there are at least 3 areas of the brain that are involved in controlling attention: frontal areas, thalamic nuclei; the posterior parietal cortex; and the interparietal sulcus. Laberge proposes that these regions are necessary for attention, and all these regions presumably give rise to attentional control together (Mancas M. , 2015).

\subsubsection{ATtENTION CONCEPT IN THE FIELD OF COMPUTER SCIENCE}

Koch and Ullman proposed that the different visual features which contribute to the attentive selection of a stimulus (colour, orientation, movement, etc.) are combined into one single topographic map, called the "saliency map" (Mancas, Ferrera, Ritche, \& Taylor, 2016). "Saliency map" is a combination between different visual features that contribute to the selection of stimuli and a single type of topographic map. The "saliency map" incorporates the normalized information from the individual feature maps into one global measure. The "saliency map" offers the probability for each region in the visual field to be attended (koch \& Ulman, 1987).

Laurent Itti in his seminal work was the first person to make the computational implementation of Koch and Ullman architecture. The first computational implementation of an attention system considers as an input any image and outputs a "saliency map" of the input image and the winner-take-all-based mechanism, simulating the eye fixations during scene analysis. This was the starting point for many developed models not only for an image but also for videos and more recently 3D (Itti \& Koch, 2001).

Another author who was interested in this branch is Matei Mancas (Mancas M. , 2015). According to Matei Mancas, attention has two approaches: the "saliency map" and the visibility. In this model when he/she needs help to perform a task, the individual looks for places where the information has good visibility. In this approach, the eyes' movement gives an automatic output of the focus area (Mancas M. , 2015).

In Computer Science, attention means that there is a filtering input space that selects the most important data in processing and this is a key mechanism of behavioral control for tasks, which is related to planning, decision-making, and 
preventing new situations. However, there are limited computation capabilities (Mancas M. , 2007), (Tamiz, Karami, Mehorabi, \& Gidary, 2013).

Attention means focusing on clear thinking, among one of several subjects or objects that may capture mind simultaneously. Attention implies the concentration of mental powers upon an object by closing or careful observing or listening, which is the ability or power to mentally concentrate (Mancas M. , 2007), (Mancas M. , 2015). In that sense, the concept of attention may be also defined as the transforming of a huge acquired unstructured data set into a smaller structured one where the main information is preserved (Mancas, Ferrera, Ritche, \& Taylor, 2016).

\subsection{FEATURES THAT INFLUENCE ATTENTION}

Generally, there are some factors that influence attention level like mental fatigue, stress, anxiety, emotions, different environment, and human health (Pimenta, Gonçalves, Carneiro, Riverola, \& Novais, 2015). Figure 3.3 presents factors that influence attention (Durães D. , Carneiro, Bajo, \& Novais, 2016).

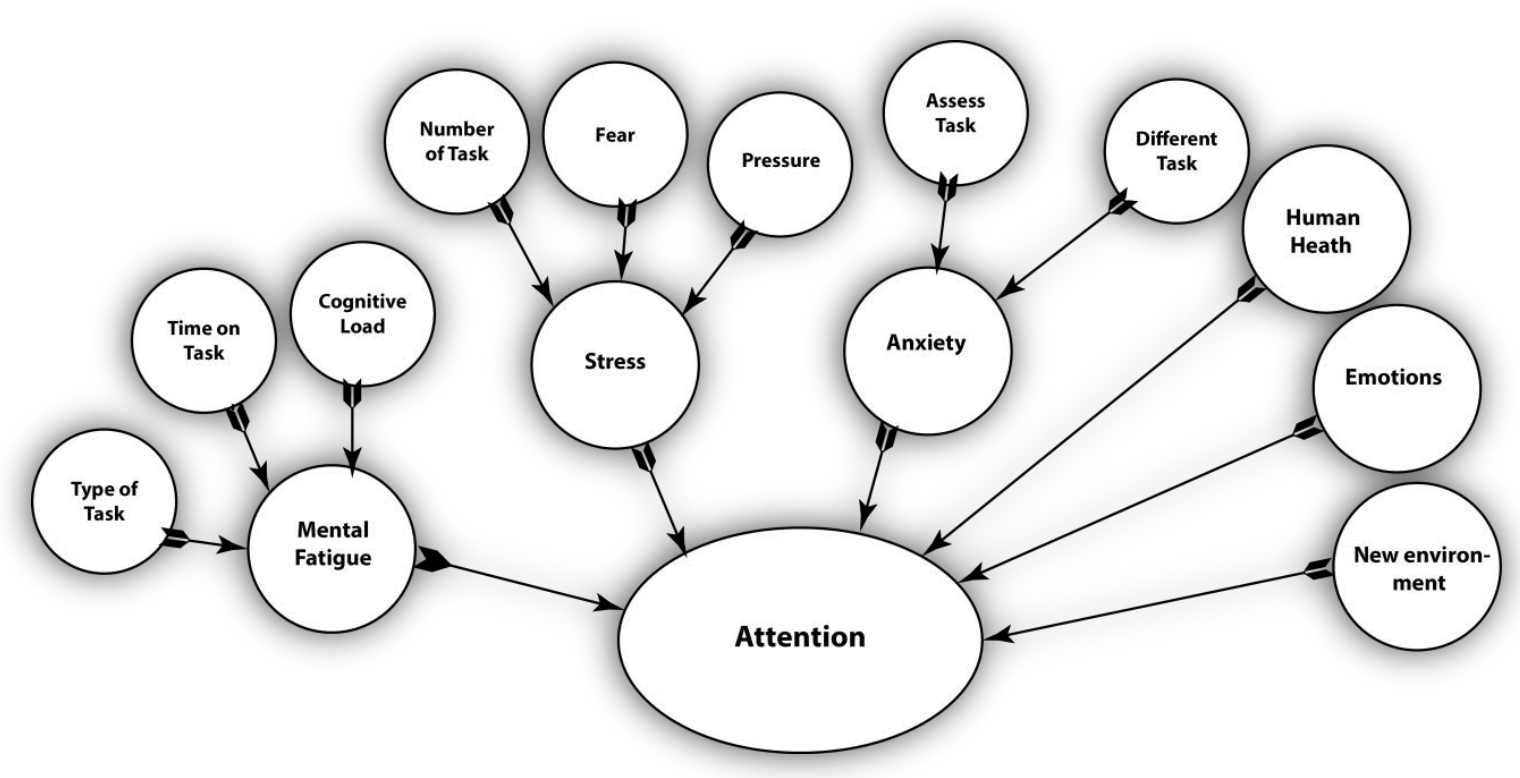

Figure 3.3 - Conceptualization of the set of factors that influence attention level. 


\subsubsection{MENTAL FATIGUE}

Usually, the term of mental fatigue is a cognitive ability that is decreased and used to describe a sequence of manifestations like lack of concentration, loss of attention, and slower reaction in response time. When students are working for an extended period of time, they often end up feeling the effect of inattention, reflected in impaired task performance and reduced engagement to continue working (Meijman, 1997), (Bartlett, 1943). In addition, one student that feels lower performance also has a harder time concentrating, getting easily distracted (Faber, Maurits, \& Lorist, 2012), (Lorist, et al., 2000), which is an indication that mental fatigue can have effects on selective attention.

Mental fatigue can be seen as the state involving effects on a set of cognitive, emotional, and motivational skills (Lorist, et al., 2000), (van der Linden \& Eling, 2006) and resulting in a person's overall malaise, as well as the emergence of a set of limitations. Some of these effects imply that a fatigued person is often less willing to engage in effort tasks, or perform the task in a conditioned way, well below their normal ability (Joyce, Blumenthal, \& Wessely, 1996), (Lorist, et al., 2000). Mental fatigue is responsible for the loss of initiative, which may be associated with an apparent lack of energy. Mental fatigue is also associated with difficulties of concentration, attention, difficulties of visual perception, and drowsiness among other problems (Neu, et al., 2010).

Mental Fatigue can occur at any time during the day. Depending on its duration and intensity, mental fatigue can make the carrying out of daily tasks increasingly hard or even impossible (Carneiro, Novais, Pêgo, Sousa, \& Neves, 2015). Learning is one of the functions that become impaired when a person is under fatigue. The importance of addressing this issue when students are using learning activities is very important for the teacher. Teachers need to be sensitive to the state of mind of their students, impairing their ability to adapt both contents and teaching strategy accordingly (Pimenta, Gonçalves, Carneiro, Riverola, \& Novais, 2015).

When some activities are prolonged for a long period of time, our brain may feel over-headed with such amount of information, and this leads to a potential emergence of mental fatigue, which decreases the level of students' attention. 


\subsubsection{STRESS}

Stress can be defined as when the pressure felt exceeds the maximum capacity of an individual can cope with (Palmer, Cooper \& $\&$ Thomas, 2003). Stress is an anomalous condition that disturbs the normal functions of body and mind. The human stress is a state of tension that is created when a person responds to a set of pressures or fears (Albastroiu, Anisia, Gonçalves, Mihaescu, \& Novais, 2018). However, a situation might be stressful for an individual and not stressful for another. For example, in the same conditions one student may feel it as a stressful one and at the same time, another student may feel it like an enjoyable situation. This means that two people are not affected in exactly the same way, or the same degree (Carneiro \& Novais, 2017). In spite of this, in some part of our life, we experience some situation of stress that can affect our body, through the change of feelings and behaviors. (Rodrigues, 2013). Figure 3.4 presents a generic diagram for representing the multi-modality space in the recognition of stress model.

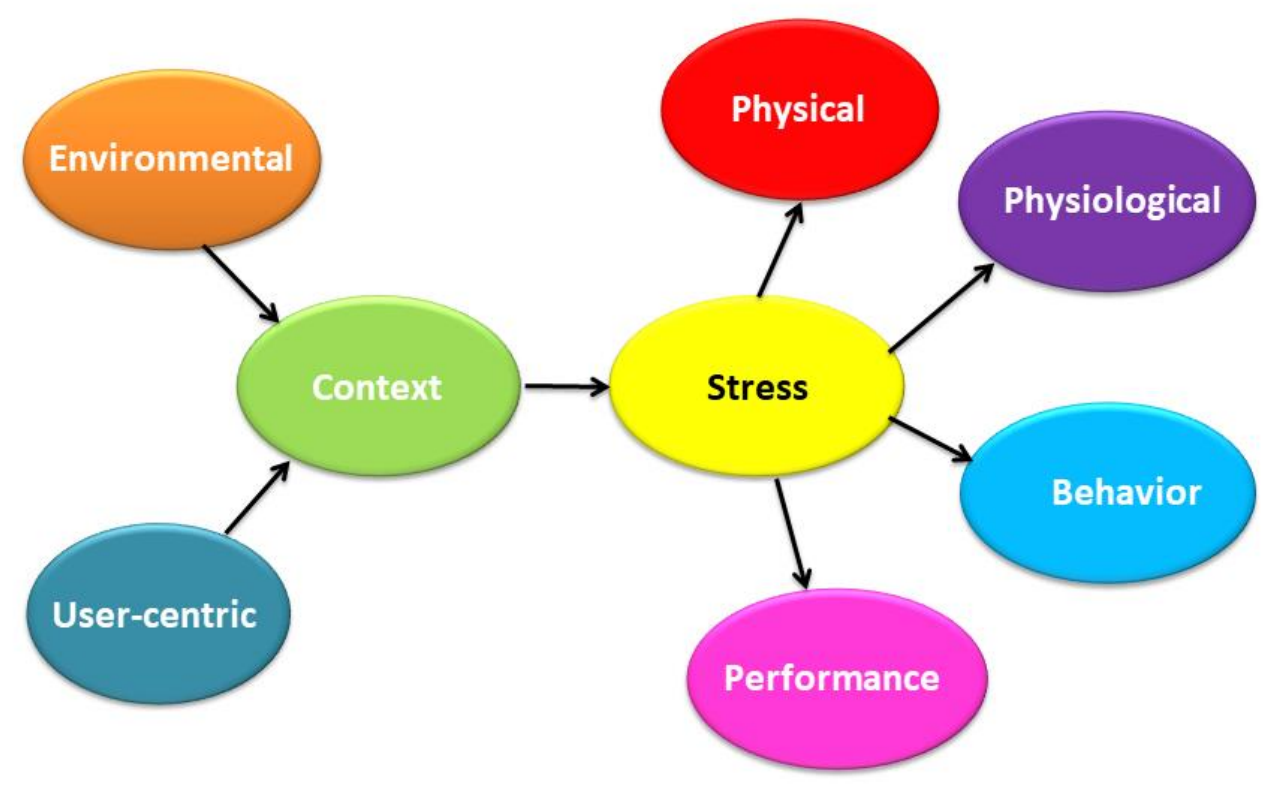

Figure 3.4 - A generic diagram for representing the multi-modality space in the recognition stress model (Rodrigues, 2013). 
Human stress is a state of tension that is created when a person responds to demands and pressures (Gardell, 1982). Students are not affected in exactly the same way or suffer from the same degree of stress. Although sooner or later in life one goes through a stressful situation (Rodrigues, Riverola, \& Novais, 2012).

Stress may have a positive or negative influence. On the one hand, it is generally accepted that stressful events increase the level of attention (Olff, Langeland, \& Gersons, 2005), (Oitzl \& Kloet, 1992), (Sandi \& Rose, Corticosterone enhances log-term retention in one-day-old chicks trained in a weak passive avoidance learning paradig, 1994) (Sandi, 1997), (Roosendaal \& MacGaugh, 1996). On the other hand, there are cases in which stressful events cause depression or anger (Shors, 2006), (McGaugh, 2004).

When students are forced to a higher number of tasks and assessment, they have to set priorities and the level of fear increases, because they don't want to fail. Consequently, the level of pressure increases causing stress. Also, if they increased the periods of work with a progressive focus on autonomy and continuous assessment, the workload is perceived as stressful and usually leads to emotional disorders, which affect attention and concentration (Carneiro, Novais, Pêgo, Sousa, \& Neves, 2015). However, in small periods of time, stress tends to behave in a more efficient way, decreasing the number of unnecessary actions making students more focused on their tasks (Rodrigues, Gonçalves, Carneiro, Novais, \& Riverola, 2013).

\subsubsection{ANXIETY}

Anxiety is an adverse emotional and motivational state occurring in threatening circumstances. State of anxiety is interactively determined by trait anxiety and by situational stress (Esysench, 1992).

A substantial literature shows that anxiety affects perceptual and related processes of attention (Bishop, 2008). Anxiety has an impact on cognition and attention because it is often associated with adverse effects on attention of cognitive tasks. Generally, anxiety has an adverse effect on attention because it causes inattention (van der Linden, Frese, \& Meijman, 2003). When, in a small period of time, it leads to compensatory strategies like enhanced effort. Anxiety changes the level of attention (Eysenck, Derakshan, Santos, \& Calvo, 2007). 
If we consider efficiency the relation between success and the resources spent on a task, anxiety is meant to have a negative influence in the field of cognition and attention through its cognitive interference by pre-empting the processing and temporary storage capacity of working memory (Esysench, 1992).

\subsubsection{EMOTIONS}

Emotions also play a fundamental role in the process of paying attention. If a person feels anxious, sad or depressed it is very hard to be concentrated and to pay attention. Furthermore, it is very hard to pay attention if people are tired, sick or feeling unwell.

Research has shown that students experience many emotions during lessons while studying, and when taking assessment and examinations (Picard, Papert, Bender, \& Blumberg, 2004). These emotions can be positive or negative, and they can change in intensity and frequency. Some of these emotions are brought into the classroom from life outside the school. Four groups of emotions are especially relevant for students' learning (Pekrun, 2014):

$>$ Achievement emotions - relate to the realization of activities and to the success and failure resulting from activities. Examples related to the success are hope and pride; related to failure are anxiety and shame.

$>$ Epistemic emotions - are especially important in learning and attention with new and non-routine tasks. Examples of this emotion are surprise, curiosity, confusion, and frustration about new obstacles.

$>$ Top emotions - belong to the topics presented in class. Both positive and negative topic emotions can trigger students' interest in attention.

> Social emotions - relate to teachers and peers in the class. These emotions are especially important in teacher/student interaction and in the group of learning, since they affect attention.

The emotions listed above can have a strong influence on learning and attention. Therefore, it is important for teachers to understand, and to deal with the emotions experienced by students. 


\subsubsection{DIFFERENT ENVIRONMENT}

The role of environment on attention is an important feature, because the physical, psychological and instructional atmosphere influences the level of attention of a person (Durães D. , Carneiro, Bajo, \& Novais, 2016).

In the physical environment, this can have a negative or a positive learning environment. Examples of negative learning environment that adversely affects student learning are low student achievement, poor behavior, student anxiety, or depression (Carneiro \& Novais, 2017).

A positive learning environment is one that allows students to feel comfortable and confident as learners. In this kind of learning environment, students are hard workers and have high achievement levels with positive energy. In order to have a positive environment, it is necessary to pay attention to the physical environment in the classroom (Carneiro \& Novais, 2017), (Durães D. , Carneiro, Bajo, \& Novais, 2016).

The use of space includes how furniture is arranged and organized, how materials are stored and maintained, how clean the classroom is and, the overall colour, luminosity, brightness, and music (Thamarasseri, 2017), (Carneiro, Pimenta, Gonçalves, Neves, \& Novais, 2015).

In a psychological environment and instructional atmosphere, it is necessary to embody an overall of attitudes. A negative attitude is not good for the student learning. The psychological environment in the classroom and instructional atmosphere in the school is how students fell about their learning. Having positive control in the school with a role model for kind words, and actions are important. Normally, students react negatively when they feel things that are unfair, unclear or are worried about getting in trouble. Students should be comfortable in the school (Thamarasseri, 2017).

\subsubsection{HUMAN HEALTH}

The human health as an important effect on attention, because problems in human health increased the irritability and strain, which lowers the attention level. The intensity with which human health affects attention depends on person to 
person. However, the level of attention is always affected (Durães D. , Carneiro, Bajo, \& Novais, 2016).

\subsection{ENGAGEMENT CONCEPT}

Engagement is one of the aspects widely used in sciences education, learning, education and psychology. Engagement is used to refer to motivational beliefs, affective states, persistence, and self-regulation.

The term disengagement is usually used to refer students' lack of persistence, curiosity and interest, lack of meta-consciousness, and negative emotions. However researchers did not have a consensus on a concrete definition and effective measurement of engagement (Sinatra, Heddy, \& Lombardi, 2015).

Some research has suggested that engagement consists of users' activities, attitudes, (Kappelman, 1995) goals and mental models, and motor skills (Said, 2004), and that it manifests itself in the form of attention, intrinsic interest, curiosity, and motivation (Chapman, 1997). "Something that engages is something that draws us in, that attracts and holds our attention." (Chapman, 1997). In learning activities, engagement is predictive of academic proficiency, motivation, and task persistence, with the balance between learner interest and task demands determining the strength of that engagement (Hoffman \& Nadelson, 2010). So, if the task is too easy or too hard for the student, student's engagement decreases. If the task is in a students' range of abilities and if it is sufficiently challenging, then students' engagement increases (Chaiklin, 2003). Nevertheless, there is a wide range of individual variation. Engagement states are the rule that arises in the application of various stimuli in different scenarios and is predictive of scholastic proficiency and results (Fox, 1997), (Posner \& Rothbart, 2007).

Engagement is vital to knowledge acquisition and the development of interest. In literature there exist three kinds of engagement: behavioral engagement, emotional engagement, and cognitive engagement (Fredricks, Blumenfeld, \& Paris, 2004), (Reschly \& Christenson, 2012).

Behavioral engagement indicates the observable aspects of engagement that range from simply following a teacher's directives to actions that require the 
student's initiative. To assess behavioral engagement, motor skills reaction time is assessed as a direct indicator of the application of the cognitive processing speed that is used during engagement with stimuli and information. The motor reaction time can be measured directly based on how quickly the student responds to stimuli on the screen (Fredricks, Blumenfeld, \& Paris, 2004).

Emotional engagement refers to students' feelings about learning components, such as teacher climate, topic, and classroom. Emotional engagement captures motivational aspects measured as expressions and experiences of states of positive emotion and negative emotion. The affective engagement is measured from different indicators. These indicators typically involve vocalizations and facial displays indicative of positive and negative emotions (Reschly \& Christenson, 2012).

Cognitive engagement refers to being focused and strategic in the learning process. Cognitive engagement includes inhibitory control of attention/control and short-term working memory (Fredricks, Blumenfeld, \& Paris, 2004), (Reschly \& Christenson, 2012).

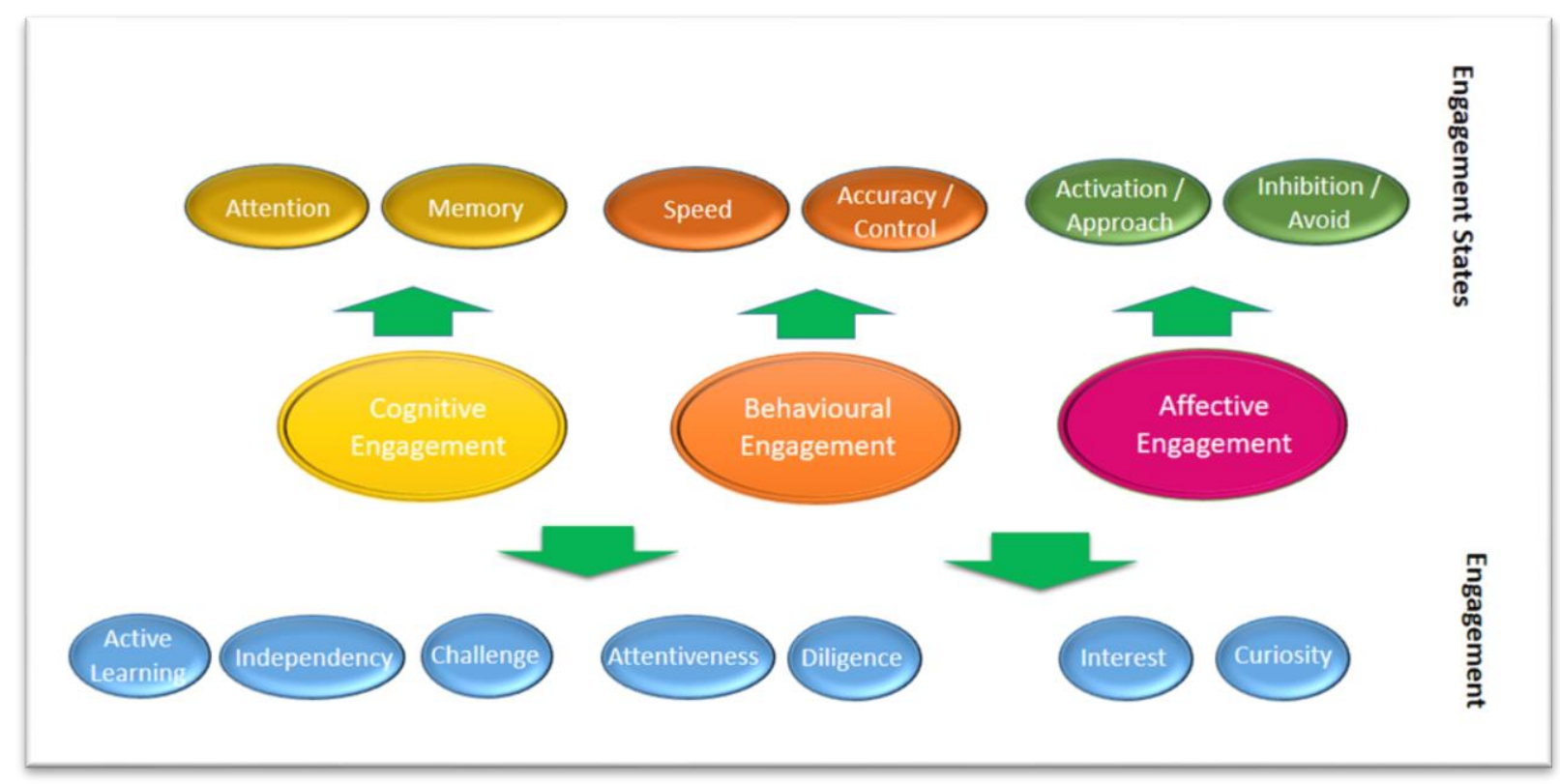

Figure 3.5 - Engagement States and Engagement (Deater-Deckard, Chang, \& Evans, 2013).

Figure 3.5 presents the various components of cognitive, affective, and behavioral engagement. Together, all type of engagement produces the broad individual differences observed. Depending on the type of engagement, there are 
some engagement states and actions. At the top of the Figure 3.5, it is presented the different indicators of engagement states. At the lower part of the Figure 3.5 it is presents the action that each engagement obtains. For cognitive engagement, the student actions are active learning, independency, and challenge. With these actions it is produced engagement state of high/lower level of attention and memory (DeaterDeckard, Chang, \& Evans, 2013).

The behavioral engagement had student actions of attentiveness and diligence. With these actions it is produced engagement state of high/lower level of speed and accuracy/control. Finally, the affective engagement had student actions of interest and curiosity. With these actions it is produced engagement state of high/lower level of actions/approach and inhibition/avoid (Deater-Deckard, Chang, \& Evans, 2013), (Fredricks, Blumenfeld, \& Paris, 2004), (Reschly \& Christenson, 2012).

For example, a web interface that is unattractive, a multimedia presentation that does not captivate the user's attention, or an online forum containing a current theme are quickly discarded with a simple mouse click. Successful technologies are not just usable, they need to engage users. Engaged users are affectively involved, motivated, and perceive themselves to be in control over the interaction.

There exist four attributes that characterized each state of engagement presented in Table 3.1.

The first one is the point of engagement, which is the initiated by the aesthetic aspect or novelty presentation of the interface. The users expressed motivation, interest, ability, and desire by the interaction of the application.

The second attribute is the engagement, which is sustained when users are able to maintain their attention and interest in the application and it is characterized by positive emotions.

The third attribute is users' disengages that occurs for many reasons, an example is the usability of the technology and distractions in their environment. 
Table 3.1. Summary of the engagement attributes (O'Brien \& Toms, 2008).

\begin{tabular}{|c|c|c|c|}
\hline \multicolumn{4}{|c|}{ Process of engagement } \\
\hline $\begin{array}{l}\text { Threads of } \\
\text { experience }\end{array}$ & $\begin{array}{l}\text { Point of engagement } \\
\text { (and reengagement) }\end{array}$ & Engagement & Disengagement \\
\hline Sensual & $\begin{array}{l}\text { - Aesthetic element and } \\
\text { pleasing or attention } \\
\text { getting } \\
\text { - Novel presentation of } \\
\text { information }\end{array}$ & $\begin{array}{l}\text { - Graphics that keep } \\
\text { attention and interest or } \\
\text { evoke realism } \\
\text { - "Ritch" interfaces that } \\
\text { promote awareness of } \\
\text { others or customized } \\
\text { views of information }\end{array}$ & $\begin{array}{l}\text { - Inability to interact with } \\
\text { features of the technology } \\
\text { or manipulate interface } \\
\text { features (usability). } \\
\text { - Lack of/too much } \\
\text { challenge }\end{array}$ \\
\hline Emotional & $\begin{array}{l}\text { - Motivation to } \\
\text { accomplish a task or } \\
\text { to have an experience } \\
\text { - Interest }\end{array}$ & $\begin{array}{l}\text { - Positive affect: } \\
\text { enjoyment, fun, } \\
\text { physiological arousal }\end{array}$ & $\begin{array}{l}\text { - } \text { Negative affect: } \\
\text { uncertainty information } \\
\text { overload, frustration with } \\
\text { technology, boredom, } \\
\text { guilt } \\
\text { - Positive affect: feeling of } \\
\text { success and } \\
\text { accomplishment }\end{array}$ \\
\hline Spatiotemporal & $\begin{array}{l}\text { - Becoming situated in } \\
\text { the "story" of the } \\
\text { application } \\
\text { - Ability to take one's } \\
\text { time in using the } \\
\text { application }\end{array}$ & $\begin{array}{l}\text { - Perception that time } \\
\text { passed very quickly } \\
\text { - Lack of awareness of } \\
\text { physical surroundings } \\
\text { - Strong awareness of } \\
\text { others when the } \\
\text { engagement revolved } \\
\text { around social } \\
\text { interaction } \\
\text { - Feedback and control }\end{array}$ & $\begin{array}{l}\text { - Not having sufficient time } \\
\text { to interact with or time to } \\
\text { devote to application } \\
\text { - Interruptions and } \\
\text { frustration in physical } \\
\text { environment. }\end{array}$ \\
\hline
\end{tabular}

Finally, the last attribute is reengagement, where the user will come again engaged. In Figure 3.6 it is presented the resume of the engagement model and attributes. 


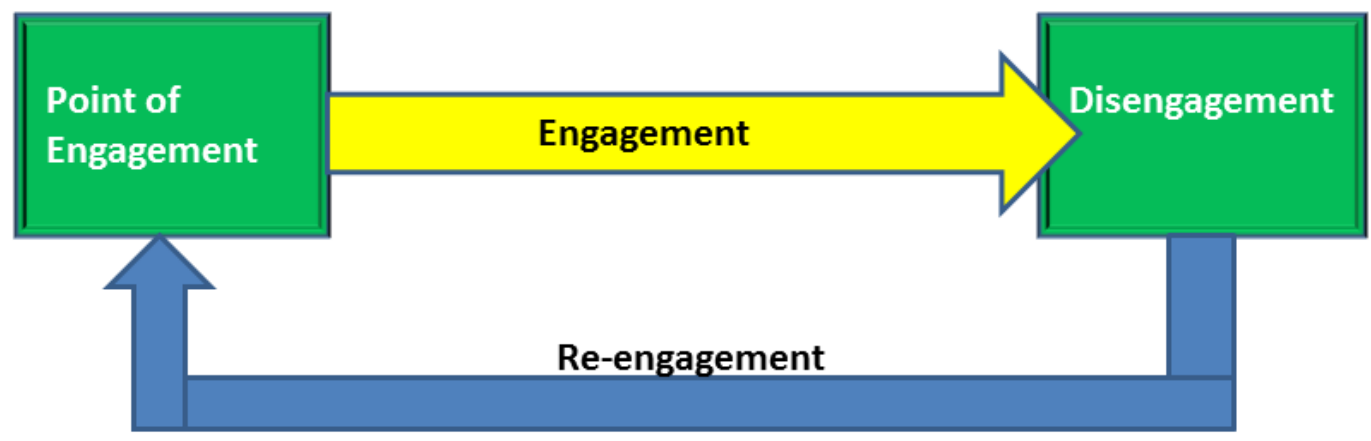

\section{Point of engagement} attributes

- Aesthetic

- Novelty

- Interest

- Motivation

- Specific or experience goal

Period of Engagement attributes

- Aesthetic and Sensory appeal

- Attention

- Awareness

- Control

- Interactivity

- Novelty

- Challenge

- Feedback

- Interest

- Positive affect
Disengagement attributes

- Usability

- Challenge

- Positive affect

- Negative affetc

- Perceive Time

- Interruptions

Figure 3.6. - Engagement model and its attributes (O'Brien \& Toms, 2008).

\subsection{LEARNING}

Learning begins a long time before school and continues for an even longer time after school, it happens quickly and in a great number of different ways and settings (Pritchard, 2009).

Different definitions of learning are presented in Table 3.2. It can also be said that learning is a process of acquire more knowledge or learning how to do something (Pritchard, 2009). Learning theories have emerged over time. However, there are three important theories: behaviorist, cognitivist and constructivist. 
Table 3.2. Some definitions of learning (Pritchard, 2009).

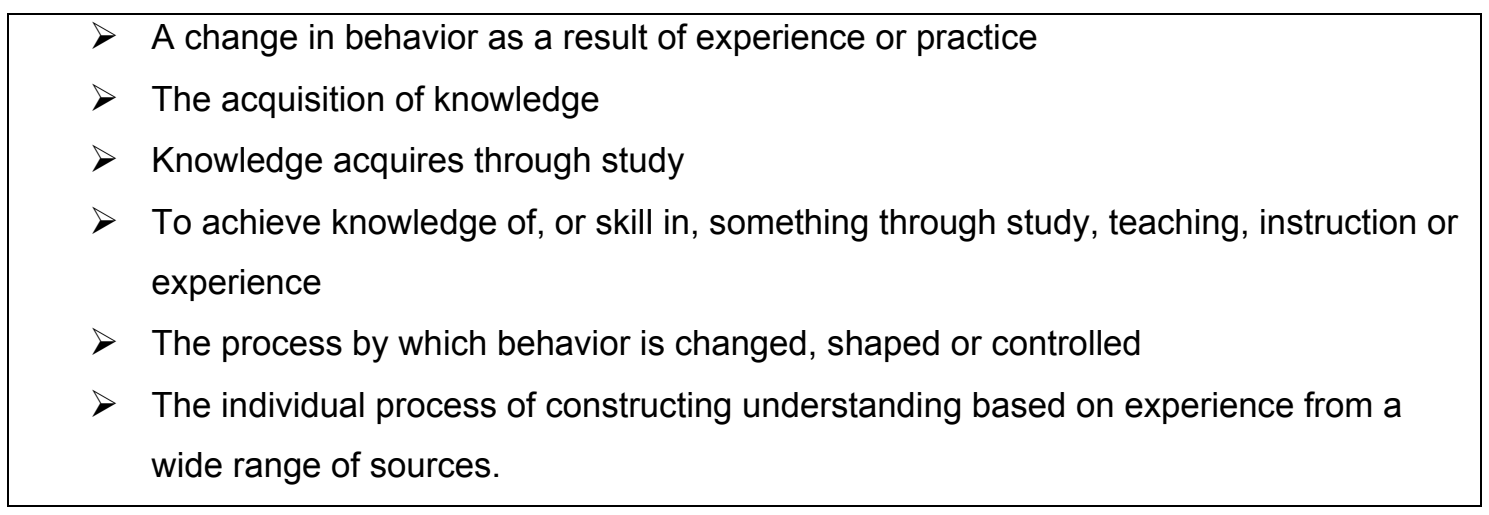

\subsubsection{BEHAVIORISM THEORIES}

Behaviorism is a learning theory focused on observable behaviors that do not consider mental activity. Learning is defined as the acquisition of a new behavior. Behaviorists call this method of learning "conditioning." Two different types of conditioning are described and demonstrated as viable explanations of the way in which animals and humans can be "taught" to do certain things.

The classical conditioning involves the reinforcement of a natural reflex or some other behavior which occurs as a response to a particular stimulus. Examples of applications of these theories are Pavlov (Coutinho, 2005), (Pritchard, 2009). Pavlov, a Russian physiologist, who conditioned dogs to salivate at the sound of a bell even without a food presence. He talks about conditioning and conditioned responses in a general way (Coutinho, 2005), (Thompson, Simonson, \& Hargrave, 1996).

Ralph Tyler (1949) also introduced for the first time, the concept of educational goals that should be defined in terms that clearly specify the type of behavior to be developed and expected on a student. It was the objectives that indicated what the student should be able to do at the end of a certain level of studies (Thompson, Simonson, \& Hargrave, 1996).

Skinner elaborates a theory of learning that became known as reinforcement theory and whose application to learning as denominate programmed learning. Reinforcement is the nuclear aspect of Skinner's theory, because it encourages the 
occurrence of appropriate behaviors and, at the same time, discourages unwanted responses (Thompson, Simonson, \& Hargrave, 1996).

Behaviorism theories are extremely useful for certain content and in very specific areas of knowledge, as well as for students with learning difficulties, since they reveal better performance in well-defined object-oriented structured environments. However, for more advanced levels of learning and with more able students, reductionism and fragmentation have managed to truly frustrating results (Coutinho, 2005).

\subsubsection{COGNITIVISM THEORIES}

Cognitive theories have shifted their focus to cognitive processes, that is, to what is happening in the subject's head between receiving a stimulus and the execution of a response.

Jean Piaget, who is considered the most influential early proponents of a constructivist approach to understanding learning, is one of the best-known psychologists in the field of child development and learning. He developed a theory called 'developmental stage' theory, which sets out age-related developmental stages. The stages begin with the sensory-motor stage (age of $0-2$ ) and end with the stage of formal operations (age of 11 or more). For Piaget, learning is a process of adjustment to environmental influences. He describes two basic processes, assimilation and accommodation, which form this process of adjustment. Assimilation is the process through which new knowledge is incorporated into existing mental structures. The knowledge bank is increased to include new information. Accommodation is the process of how mental structures have to be changed in order to cope with the new experience which has opposed to the existing model. Equilibration is the process of received at a stable state where there is no longer a conflict between new and existing knowledge (Pritchard, 2009).

Cognitive psychologists refer to units of knowledge, understanding and skill as schemas, as a way of referring to conceptual knowledge which is stored in longterm memory. It is predictable that any adult person would have hundreds of thousands of schemas in memory, which would be interrelated in an extremely large 
and complex number of different ways. New schemas are created every time and one fact is linked to another by a logical or semantic connection (Pritchard, 2009).

\subsubsection{CONSTRUCTIVIST THEORIES}

The key protagonists of this branch of constructivism are Vygotsky, a Russian whose work was carried out at the begin of the twentieth century. In constructivist theory, the emphasis is placed on the interaction between the learner and others. Others can come in many forms - they are the dimension of social interaction that is crucial to social constructivists. Learning happens from social interaction and it can be established with the individual rebuilds his knowledge due to the mixture between personal construction processes and processes of collaboration with other stakeholders. In this last process, the teacher/tutor should be an agent that facilitates and mediates the culture (Pritchard, 2009), (Coutinho, 2005).

Constructivists learning is an active process of building, not acquiring knowledge and the purpose of the instructional process is to help this construction, not transmit knowledge. In this perspective, it is the student who has the lead role: he becomes the center of the process and all the other elements - teacher, content, media, and environment - make sense only if they help to create conditions for the learner to build the knowledge. Dialogue becomes the vehicle by which ideas are considered, shared and developed and the teacher has the role of stimulating dialogue. In a very real way, the teacher engages groups and individuals in dialogue and supports the development of understanding (Pritchard, 2009), .

There are seven key concepts in a constructivist conception of learning: learning by discovery, a zone of proximal development (ZPD), situated learning, collaborative learning, cognitive flexibility, goal-based scenarios (GBS), and anchored instruction (Coutinho, 2005).

Learning by discovery consists of learning-to-learn theory, including the ability to ask questions, evaluate personal strategies, and propose reasoned answers (Coutinho, 2005).

The ZDP was defined by Vygotsky as a form of shared cognition in which the teacher/tutor supports the learner until the learner appropriates the knowledge or skill and eventually becomes independent (Coutinho, 2005), (Carvalho, 2000). 
Situated learning that emphasizes the idea that learning is a function of the task, context, and culture in which it occurs, in other words, it is "situated" (Coutinho, 2005).

Collaborative learning is a form of cultural learning in which people in talking to each other to build knowledge through a successful negotiation of consensus (Coutinho, 2005).

Cognitive flexibility focuses attention on learning that occurs in domains of knowledge that are characterized by being complex and malformed. We can say that cognitive flexibility is the ability of the subject to be able, in face of a new situation, to be able to restructure the knowledge he already possesses in order to solve it (Carvalho, 2000).

GBS is a theory of memory and learning developed by artificial intelligence, in a simulated "learning by doing" environment. It consists in carrying out a task in which the student pursues goals using for this purpose the knowledge he/she already has in the realization of interesting and motivators problems. During the simulation, the student receives the coaching that is strictly necessary so that he/she can use the available information in an effective way (Coutinho, 2005), (Carvalho, 2000).

Anchored instruction is the development of interactive videos that encourage students and professors to pose and solve complex but realistic problems. Video materials serve as "anchors" for all learning activities (Coutinho, 2005).

\subsubsection{LEARNING PROCESS}

The learning process research has been focused on different aspects, according to the different psychology branches referred to in the previous subsection. Nowadays, learning uses a mixture of the three theories referred in the previous subsection, so independent of the theory, the learning process is a cycle with different steps (Bybee, 1989), (Rodrigues, Novais, \& Santos, 2005).

To explain how learning is processed it is possible to use the learning cycle shown in Figure 3.7. The learning cycle has five steps: Engagement, Exploration, Explanation, Elaboration, and Evaluation (Bybee, 1989). 
The first step of the learning cycle is the engagement step where the student's attention is focused on the subject. In this step, prior knowledge is explained, and the student is reminded about topics that she/he should already be familiar with. The evaluation of this step consists in pre-assessing the prior knowledge of the subject (Bybee, 1989), (Durães, Bajo, \& Novais, 2017).

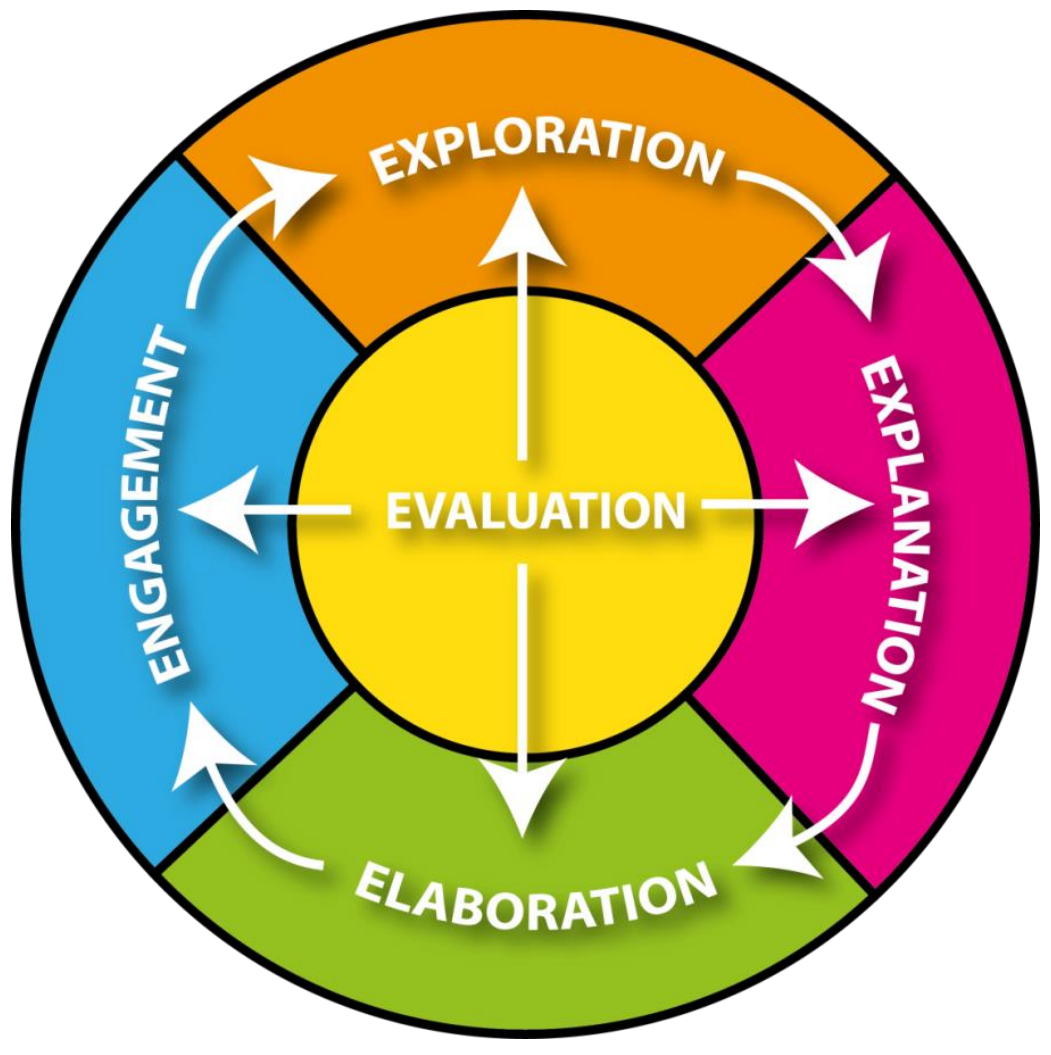

Figure 3.7 - Learning cycle (Bybee, 1989).

The second step, the exploration step, is to have students gathering information that they can use to solve the problem that was proposed. The evaluation of the exploration step is carried out through the evaluation of the gathering information process (Bybee, 1989), (Durães, Bajo, \& Novais, 2017).

In the explanation step, the students use the gathered information to solve the problem and report what they have done and also try to work out the answer to the presented problem. The evaluation of explanation focuses on how well the students are using the gathered information and what new ideas they have come up with (Bybee, 1989), (Durães, Bajo, \& Novais, 2017). 
The elaboration step is where the student has new information that extends what they have been learning. Also, in this stage students are solving problems that require the knowledge acquired during the learning process in order to solve them. The evaluation of the elaboration step usually is the test at the end of the subject, which measures how well students understood what they have learned (Bybee, 1989), (Durães, Bajo, \& Novais, 2017).

\subsection{LEARNING STYLES}

Learning style is defined by many useful definitions and related ideas. Some examples of these definitions are (Pritchard, 2009), (Durães D. , Carneiro, Jimenez, \& Novais, 2018):

A particular way in which an individual learns;

$>$ A method of learning - an individual's preferred or best manner(s) in which to think, process information and demonstrate learning;

> An individual's preferred means of acquiring knowledge and skills;

$>$ Habits, strategies, or regular mental behaviors concerning learning, particularly deliberate educational learning, which an individual displays.

A learning style is a method that allows an individual to learn best. Different people learn in different ways, each one preferring a different learning style. Everyone has a mix of learning styles, but some people may find that they have a dominant style of learning. Others may find that they have different learning styles in different circumstances. There are several models developed by several authors that try to represent the way people learn (Morgan, Baker, \& Alabama, 2013).

Learning styles can be defined as cognitive, affective, and physiological features that serve as relatively stable indicators of how learners perceive interaction and respond to their learning environments (Keefe, 1979).

Learning style not only specifies how a student learns and likes to learn, but it can also help a teacher to adapt to individual students so that they might learn successfully. When teacher's methodologies do not support a specific learning style, a student will find it more difficult to learn and acquire knowledge. When students are doing learning activities using new technologies, it is extremely important that 
teacher has feedback from the students' work in order to detect potential learning problems at an early stage so he/she can choose the appropriate teaching methods (Durães D. , Carneiro, Jimenez, \& Novais, 2018), (Morgan, Baker, \& Alabama, 2013).

Previous research suggests that, in the context of learning activities, different learning styles can influence learning performance. Learning styles is also considered one of the more important factors influencing learning (Ford \& Chen, 2000). Some researchers have argued that learning style is also a suitable indicator of potential learning success because it provides information about individual differences in learning preferences and information-processing (Kolb D. , 1984), (Smith \& Ragan, 1999).

Learning is improved if the teacher has a system that can detect and classify the learning preferences of students and provide advice from potential learning problems at an early stage in order to choose the most appropriate teaching methods.

The learning style study is very controversial because some authors think that the many existing theories are very close and their scientific support is lacking. For these authors, the learning style is not a straight concept, which means, that if a person prefers or feels more comfortable with the perception style, this not means that tomorrow the same person will not prefer an understanding style to learn (Durães D. , Carneiro, Jimenez, \& Novais, 2018).

\subsubsection{KOLB'S LEARNING STYLE MODEL}

Kolb expressed his principle that a person learns from discovery and experience (Kolb \& Kolb, 2005). For Kolb, an individual does not learn by reading or observing, it is necessary to experience. His theory proposes four distinct learning styles that are based on four stages of the learning cycle (Kolb \& Kolb, 2005).

The learning cycle is so divided into four categories: Concrete Experience, Reflective Observation, Abstract Conceptualization, and Active Experimentation. The cycle begins with Concrete Experience and in this phase corresponds to the beginning of a task. Then, the Reflective Observation stage is the moment to stop the task and think about what was done and what was experienced. The third 
category is Abstract Conceptualization, which the individual interprets what occurred and the relations between the conditions. The final category is Active Experimentation, which is considered the way to put into practice what has been learned. In Figure 3.8 it is presented the result of this theory, which was a set of learning styles that satisfy the human's preferences and a learning style cycle (Kolb D. , 1984).

After defining the learning cycle, Kolb defined four learning styles based on the cycle described above. To Kolb, each person is different in the way of learning and several factors, such as social environment, basic cognitive structure, and educational experience influences his/her learning style. The learning style is a product from two variables: Processing Continuum, responsible for the way to approach a task, and Perception Continuum, relates to the feelings towards a task (Kolb D. , 1984), (Kolb \& Kolb, 2005).

Kolb's learning styles model classifies individuals over two dimensions as having a preference for (Pritchard, 2009):

$>$ The first dimension - is the dimension concerning how the learner takes information. There are two modes, the concrete experience mode or the abstract conceptualization mode.

$>$ The second dimension - is the dimension concerning how the learner incorporates information. There are also two modes, the active experimentation mode or the reflexive observation mode.

Kolb describes four general learning styles based on the two dimensions presented in Figure 3.9, as follows (Pritchard, 2009):

Type 1: Diverger (concrete, reflective). Diverging learners are imaginative and sensitive and prefer to learn by observation, gathering information, and brainstorming. This type of person often uses the question "Why" and they perceive through concrete experience (CE) and a process by reflective observation (RO). They have the capacity to produce and/or assimilate various observations for new idea generation. They are less concerned with theorems and generalizations, so their approach to problem-solving is not systematic but is more creative in comparison to the other learning styles. These learners when working in groups 
listen to the suggestions of others and accept critiques from them (Kolb \& Kolb, 2005).

Type 2: Assimilator (abstract, reflective). Assimilating learners benefit if they are given time for reflection and they prefer to learn by placing information in a concise logical order and using reflective observation. This type of person often uses the question "What?" and responds well to information presented in an organized, logical style. Assimilating learners notice through active conceptualization (AC) and a process by reflective observation (RO). They are more concerned with abstract concepts rather than practical applications. These learners choose readings, lectures and exploring analytical models (Kolb \& Kolb, 2005).

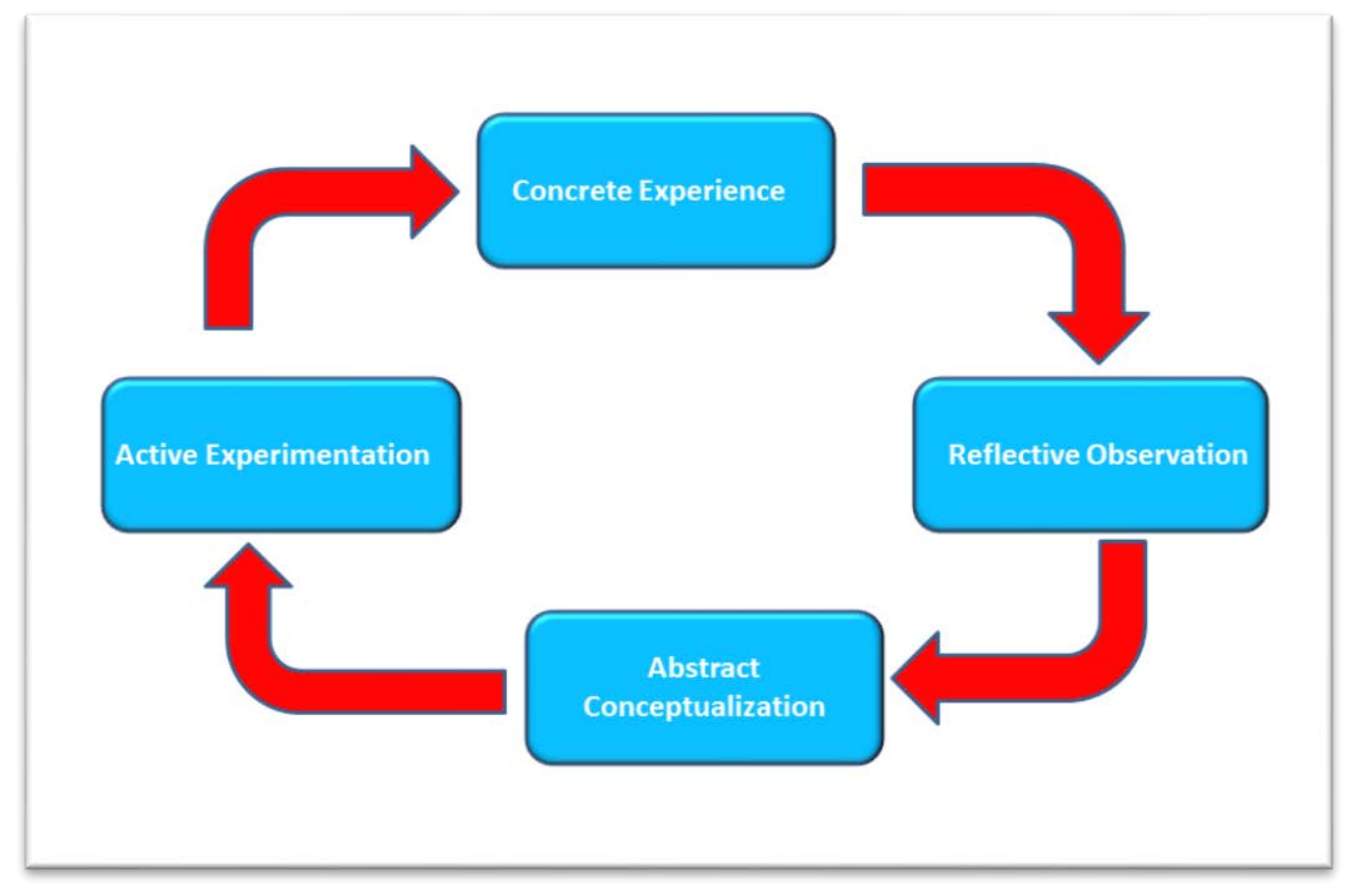

Figure 3.8 - Kolb Learning Cycle (Kolb D. , 1984).

Type 3: Converge (abstract, active). Converging learners learn by trial and error in an environment that allows them to fail safely. They like to learn by solving problems and doing technical tasks and they are good at finding practical uses for ideas. This type of person often uses the question "How?" and answers to opportunities to work actively on well-defined tasks. Converging learners perceive through active conceptualization (AC) and a process by active experimentation (AE). These learners bring a logical, pragmatic and unemotional perspective to the 
problem-solving process and prefer to focus on things rather than people. Their knowledge is structured and they do hypothetical-deductive reasoning while centring on a specific problem (Kolb \& Kolb, 2005).

Type 4: Accommodator (concrete, active). Accommodating learners are people-oriented, hands-on learners and rely on feelings rather than logic. This type of person often uses the question "What if?" and answers well when they are able to apply the new material in problem-solving situations. Accommodating learners perceive over concrete experience (CE) and a process by active experimentation $(\mathrm{AE})$. They like to take risks and they enjoy finding out new experiences. They solve problems using a trial-and-error method rather than using their analytical abilities. These learners also depend on others for information and they prefer to work with others to do tasks, to set goals, to do fieldwork and to test various approaches for design alternatives (Kolb \& Kolb, 2005).

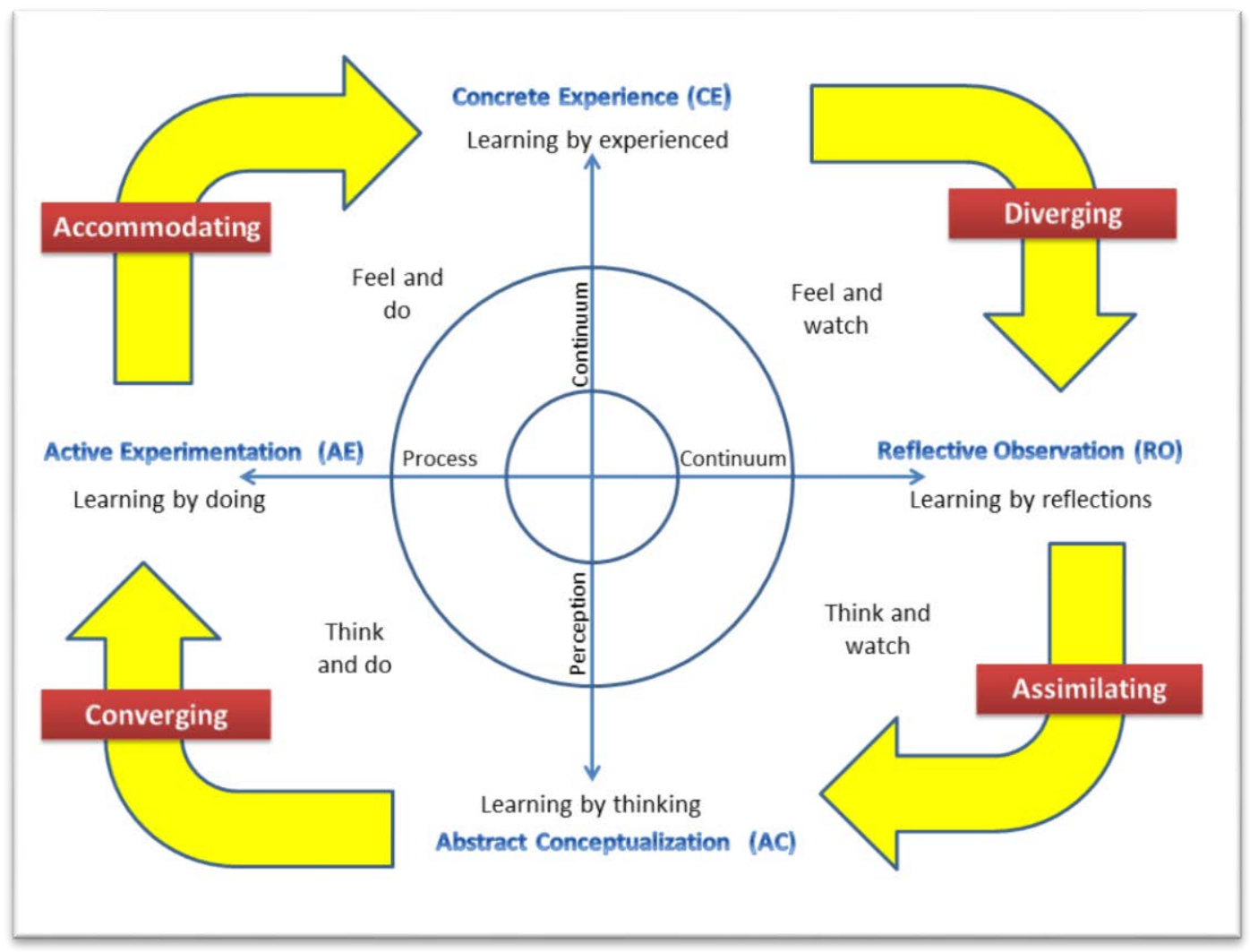

Figure 3.9 - Kolb's Learning Style (Kolb \& Kolb, 2005). 


\subsubsection{HONEY AND MUMFORD LEARNING MODEL}

Honey and Mumford (1986) propose that we need to be able to adopt one of four different styles in order to complete any given learning task. The four styles described in the Honey-Mumford Model are activists, reflectors, theorists, and pragmatists.

The activist style corresponds to people who learn with practical activity and have an open mind to learn. They prefer to learn by doing rather than, for example, by reading or listening. Activities like competitions, brainstorming and group discussion are their preferences and they thrive on novelty and to immerse themselves in a wide range of experiences, activities, and like to work in groups so that ideas can be shared and ideas tested. They like to get on with things, so they are not interested in planning. Activists are irritate by repetition, and are most often open-minded and enthusiastic (Pritchard, 2009).

The reflector style shows people who prefer to observe and think about what they saw. They prefer activities like observations, conferences, and coaching. They like to accumulate as much information as possible before making any decisions. They prefer to look at the global problem, including previous experiences and the perspectives of others. Reflectors are slow to take their decisions, but when they do, they are based on both their own knowledge and opinions and on what they have taken by listening thoughts and ideas of others (Pritchard, 2009).

The theorist is the learning style for people who like to understand where the new knowledge will fit into what they already know. They like to adapt and integrate all of their observations into frameworks so that they are able to see how one remark is related to other. Furthermore, they have tidy and well-organised minds and they are uncomfortable with anything subjective or ambiguous. Theorists are typically rigorous in their approach to problem-solving, taking a logical, one-step-at-a-time approach (Pritchard, 2009).

The pragmatics are people whose learning style are interested in real facts instead of abstract theories. These people prefer activities like case studies, discussion, and time to think about how to apply what they learn. Pragmatists search for the practical consequences of any new ideas or theories before making a decision on their value. The philosophy of the pragmatists is that if something works 
then all is well and good if something does not work, they desperately spends time on the analysis of its failure (Pritchard, 2009). In Figure 3.10 it is present the Honey and Mumford learning model.

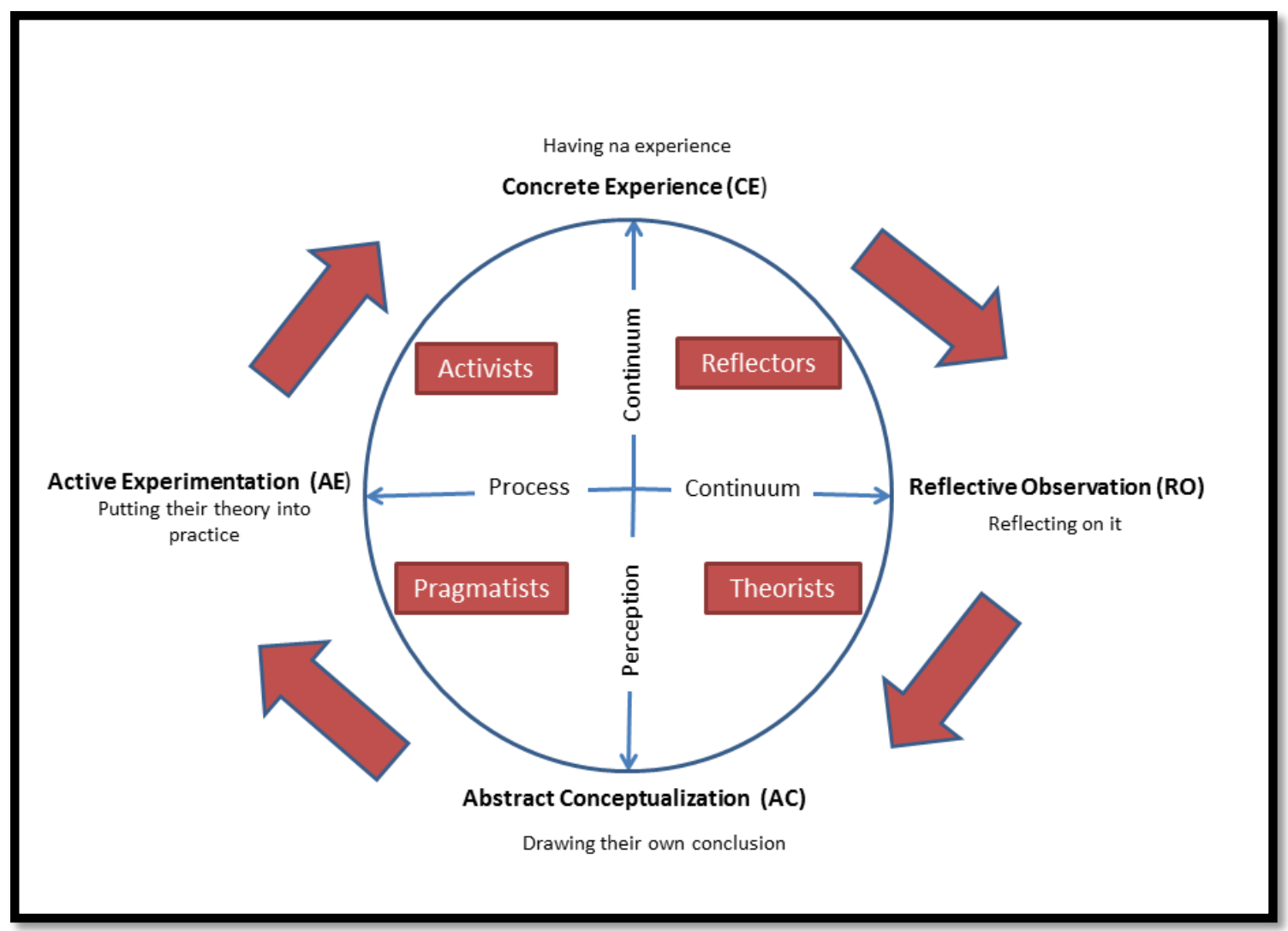

Figure 3.10 - Honey and Mumford learning model (Honey \& Mumford, 2006).

In Table 3.3 it is presented the Peter Honey and Alan Mumford theory developed about learning styles based on Kolb's model.

Table 3.3. Comparison between Honey and Mumford model and Kolb model.

\begin{tabular}{|c|c|}
\hline Honey and Munford & Kolb \\
\hline Activist & Accommodating \\
\hline Reflector & Diverging \\
\hline Theorist & Assimilating \\
\hline Pragmatics & Converging \\
\hline
\end{tabular}




\subsubsection{FELDER-SILVERMAN LEARNING MODEL}

This theory considered different dimensions of learning. In addition, learning in a structured educational environment can be thought of as a two-step process involving receiving and processing information. In the reception step, external information (visible through the senses) and internal information (arising introspectively) become available to students, who select the material they will process and ignore the rest. The processing step can involve simple memorization or inductive or deductive thinking, reflection or action, and introspection or interaction with others. The result is that the material is either acquired in one sense or another or not acquired. This theory does not have only the function to help students understand how they can improve their learning but also, to give some teaching techniques for each learning style proposed.

The learning styles were based on other theories from different authors, such as Jung's and Kolb (Felder \& Silverman, 2002). The styles were also based on students' preferences about the way they enjoy receiving and process the new information. The dimensions used to characterize the learning styles were (Felder \& Silverman, 2002):

$>$ Processing: Active or Reflective.

$>$ Perception: Sensing or Intuitive

$>$ Input: Visual or verbal

$>$ Organization: Inductive or Deductive

$>$ Understanding: Sequential or Global

The first dimension distinguishes between an active and a reflective way of processing information. Active learners learn best by working actively with the learning object, and by trying things out. Furthermore, they tend to be more interested in communicating with others and prefer to learn by working in groups where they can discuss about the learned object. In contrast, reflective learners prefer to think about and reflect on the object. Regarding communication, they prefer to work alone or in a small group (Felder \& Silverman, 2002).

The second dimension distinguishes between sensing and intuitive learning. Students who prefer a sensing learning style like to learn facts and they tend to be very patient with details and solve the problems with standards approaches. This 
type of students is considered to be more realistic than sensible. Another characteristic is that they like to relate the learn object to the real world. Intuitive learners prefer to learn abstract things, such as theories. They are more able to discover possibilities and relationships, so they tend to be more innovative and creative than sensing learners (Felder \& Silverman, 2002).

The third dimension distinguishes between visual or verbal learners. This dimension differentiates learners who remember best and therefore prefer to learn from what they have observed. This type of learns like to learn with pictures, diagrams, and flow-charts. Verbal learners prefer more textual representations, such as written or spoken texts (Felder \& Silverman, 2002).

The fourth dimension distinguishes between inductive and deductive learners. Inductive learners like the progression that proceeds from particulars (observations, measurements, and data) to generalities (governing rules, laws, and theories). In opposite, deductive learners proceed in the opposite direction. In induction one infers principles; in deduction one deduces consequences (Felder \& Silverman, 2002).

The fifth dimension distinguishes between sequential or global learners. Sequential learners learn in small incremental steps and therefore have a linear learning progress. In opposite, global learners use a holistic thinking process and learn on a large scale. They tend to absorb the learning object almost casually without seeing connections but after they have learned enough objects they quickly get the whole picture (Felder \& Silverman, 2002).

There are $32\left(2^{5}\right)$ learning styles in the proposed conceptual framework. This happens because each style was described for an option from each dimension. For example, one style is the active/intuitive/verbal/deductive/sequential. If this approach was used in an educational context, the teacher had to teach a subject in 32 possible ways, which was impracticable with the available time. To solve this problem, the usual methods of engineering education adequately address five categories: intuitive, auditory, deductive, reflective e sequential as we can see in Table 3.4. 
Table 3.4 - Felder-Silverman Learning Styles and Teaching Style (Felder \& Silverman, 2002).

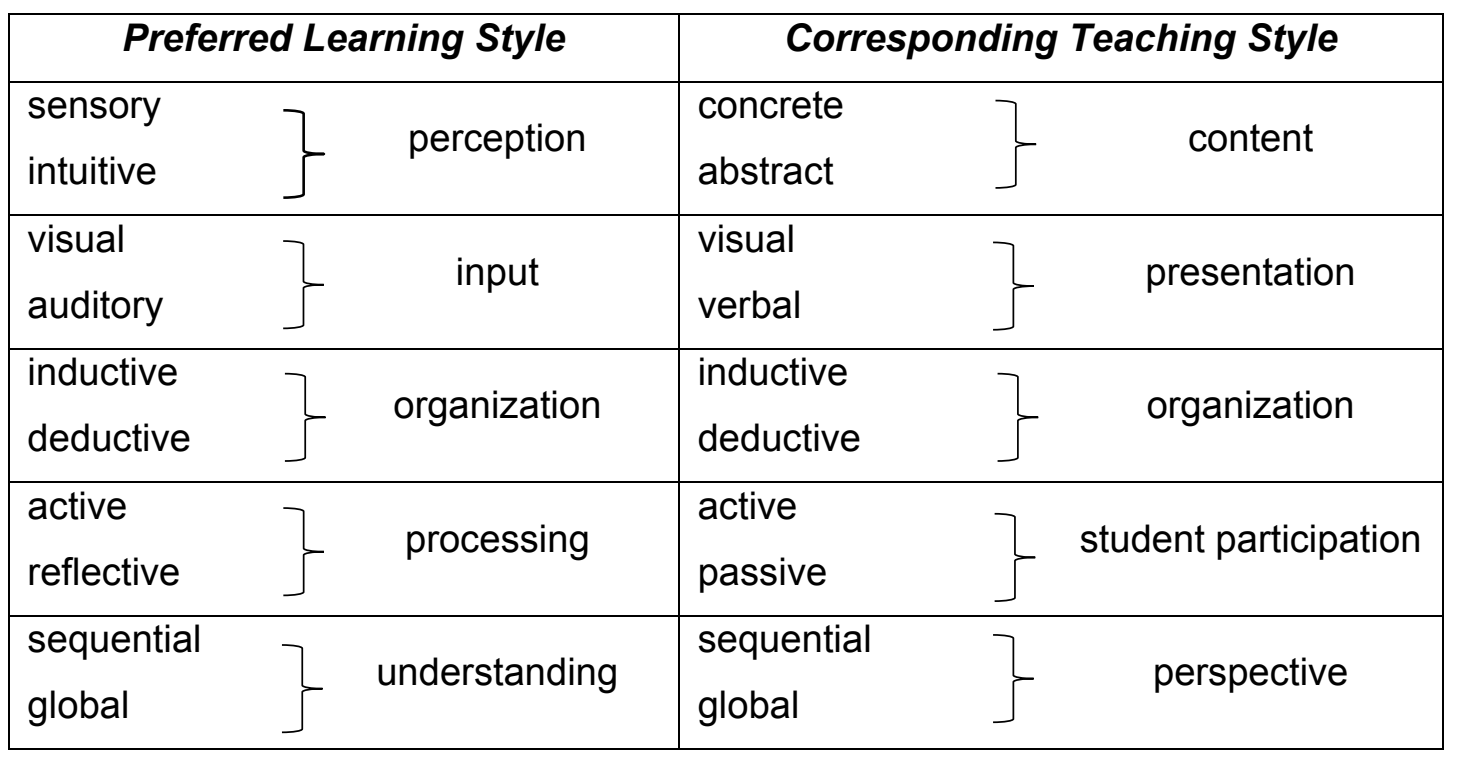

\subsection{SUMMARY}

In Computer Science, attention means that there is a filtering input space that selects the most important data in processing and this is a key mechanism of behavioral control for tasks, which is related to planning, decision-making, and preventing new situations. However there are limited computation capabilities (Mancas M. , 2007), (Tamiz, Karami, Mehorabi, \& Gidary, 2013). Attention means focusing on clear thinking, among one of several subjects or objects that may capture mind simultaneously. Attention implies the concentration of mental powers upon an object by close or careful observing or listening, which is the ability or power to mentally concentrate (Mancas M. , 2007), (Mancas M. , 2015). In that sense, the concept of attention may be also defined as the transforming of a huge acquired unstructured data set into a smaller structured one where the main information is preserved (Mancas, Ferrera, Ritche, \& Taylor, 2016).

However, there are some factors that influence attention level like mental fatigue, stress, anxiety, emotions, different environment, and human health (Pimenta, Gonçalves, Carneiro, Riverola, \& Novais, 2015), (Durães D. , Carneiro, Bajo, \& Novais, 2016). 
Engagement is one of the aspects is widely used in sciences education, learning, education, and psychology. Engagement is used to refer to motivational beliefs, affective states, persistence, and self-regulation. The term disengagement is usually used to refer students' lack of persistence, curiosity and interest, lack of meta-consciousness, and negative emotions. However researchers did not have a consensus on a concrete definition and effective measurement of engagement (Sinatra, Heddy, \& Lombardi, 2015). Some research has suggested that engagement consists of users' activities, attitudes, (Kappelman, 1995) goals and mental models, and motor skills (Said, 2004), and that it manifests itself in the form of attention, intrinsic interest, curiosity, and motivation (Chapman, 1997). "Something that engages is something that draws us in, that attracts and holds our attention." (Chapman, 1997). In learning activities, engagement is predictive of academic proficiency, motivation, and task persistence, with the balance between learner interest and task demands determining the strength of that engagement (Hoffman \& Nadelson, 2010). So, if the task is too easy or too hard for the student, students' engagement decreases. If the task is in a students' range of abilities and if it's sufficiently challenging, then students' engagement increases (Chaiklin, 2003). Nevertheless, there is a wide range of individual variation. Engagement states are the rule that arises in the application of various stimuli in different scenarios and is predictive of scholastic proficiency and results (Fox, 1997), (Posner \& Rothbart, 2007).

Nowadays, learning uses a mixture of the three theories referred to in the subsection, 3.4 so independent of the theory, the learning process is a cycle with different steps (Bybee, 1989). Learning is a process where the human being captures and processes information and creates knowledge. This process may take place in a more formal way like school, or through personal experience. All situations can lead to knowledge but some situations are more propitious for learning (Macarro, Villafaina, Pinto, \& Bajo, 2011). One of the reasons is the possibility to create strategies in order to facilitate the learning and engaged people attention.

In education, the definitions of learning styles and teacher's techniques have been the main factors in promoting interest in school and in improving the student's school achievement. Learning styles have been much studied by several authors, such as Kolb, Honey, Mumford, Felder, and Silverman. The theory of learning styles 
that we will be followed is the Felder-Silverman theory because it is a theory based on all the previous ones and it is more complete and flexible. Another aspect is that this theory considered different dimensions of learning. This theory does not only have the function to help students understand how they can improve their learning, but also, gives some teaching techniques for each learning style proposed (Felder \& Silverman, 2002).

Until now it was presented the state of art of concepts necessary for the development of this thesis. Based on all of these concepts, the next chapter, 3S's Architecture, outlines the project and system architecture designed and developed, where are specifies the technologies used and their implementation details. 
"Technology has become as ubiquitous as the air we breathe, so we are no longer conscious of its presence." Godfrey Reggio

\section{4}

\section{3S'S ARCHITECTURE}

After the definition of the research problem and its main objectives, the next step is the definition and development of a new architecture. So, to find out the level of attention that influences the learning process it was necessary to have a framework which captured data from students. Based on these captured data, and after analyzed and treated, the idea is to create a system that creates an user profile and warns the teacher about: levels of attention, learning style, and user's behavior. So that helps the teacher to adjust and adapt the level of learning of each student in different situations. According to the background research, not only this adaption should be performed based on the student's emotions, but also based on other student's characteristics like personality and learning preferences (see section 3.5). The inclusion of these characteristics helps the teacher to understand the information presented making possible enhancing the learning results.

The development of a new architecture occurs due to the void found in the literature review since most systems developed until now are invasive and intrusive (see section 2.5). While there are some monitoring systems of attention level, they do not fit the initial objectives of this doctoral thesis. Hence it is required to develop a new model to answer to the hypothesis initially proposed. So, the idea is to create a non-intrusive and non-invasive approach, from the observation of changes of an individual or a class, concerning behavioral pattern in order to predict student's behavior in (e)learning lessons in order to improve learning. Figure 4.1 presents a 
general view of how we want to improve learning based on attention as a form of engagement.

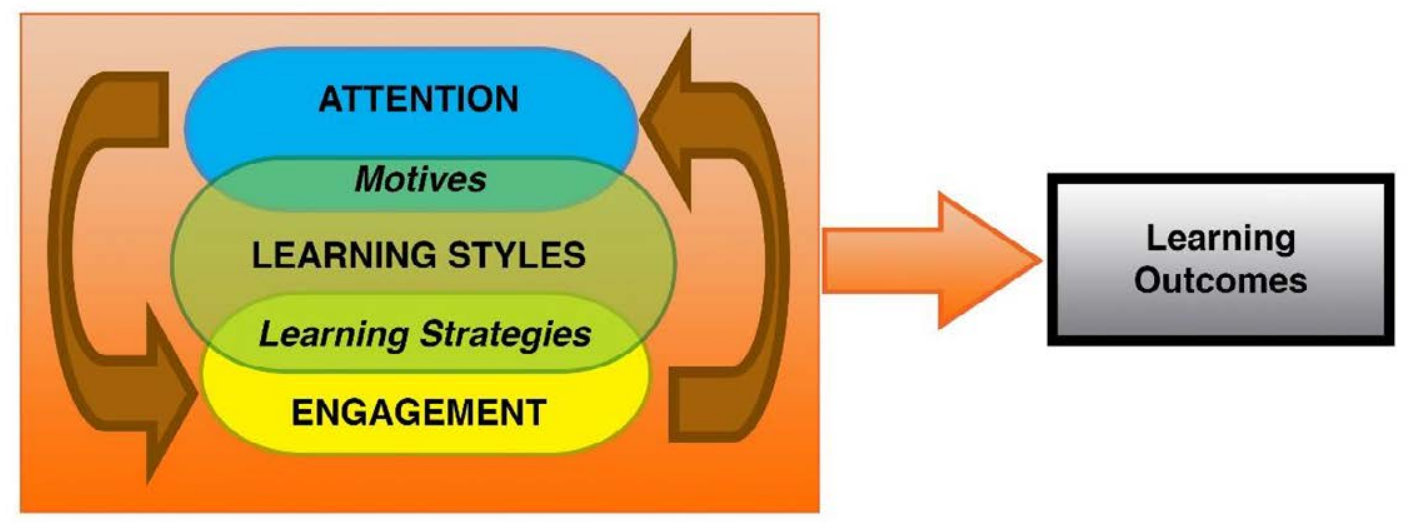

Figure 4.1 - Attention as form of engagement.

\subsection{NEW APPROACH}

As it was stated before this research deals with the issue of attention monitoring, with the aim of providing a non-intrusive, reliable and easy framework that can be used freely in schools or organizations, without changing or interfering with the established working routines. Specifically, we look at desk students in learning activities, in which the student spends a long time interacting with the computer. It is especially suited to students working with computers and can be interesting for domains such as the workplace or the classroom.

The idea is that the proposed framework constantly analyzes the behavior of the user while he/she interacts with the computer. Using this results and with knowledge about the task, the idea is to classify the level of attention, the user's learning style and the user's interaction behavior. Finally, with this information, it is possible to predict features and help in decision-making. One of our guiding lines is that such framework will be non-intrusive and non-invasive since less intrusive techniques facilitate more accurate and frequent monitoring.

Figure 4.2 presents an overview of the strategy followed on this project in which we can observe the existence of 3 layers of abstraction (Carneiro \& Novais, 2017). The first layer is from the interaction with different devices, the extraction of knowledge in different ways is performed for server decision support in different 
environments. Currently, there is a huge diversity of devices that assist in performing tasks in the workplace or in a teaching-learning context. These devices are smartphones, tablets, and computers and they are completely disseminated in society. In a non-intrusive and non-invasive way, the model collects data from the interactions of the users. The second layer acts based on this interaction, data are collected, and the model should analyse human behavior patterns and the levels of attention. Finally, the third layer with this information is possible to executes decisions and establish intervention strategies so that the desired levels of attention can be achieved in the context in which the analysis is carried out.

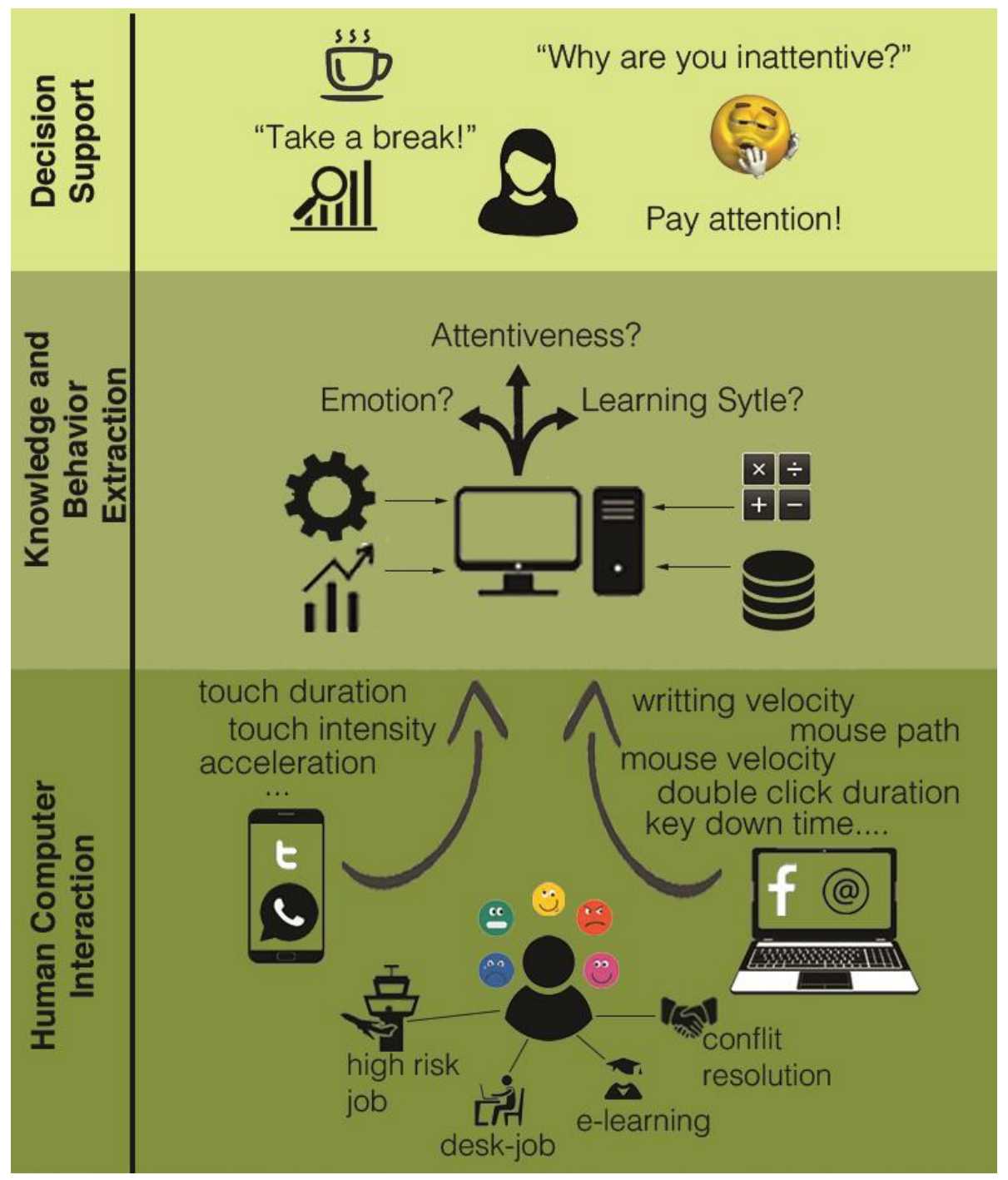

Figure 4.2 - new approach model. 
The extraction of knowledge and emotion is an important step because it allows the prediction of future user behavior that can affect the learning process. This possibility is evidenced as essential in realities where the lack of direct contact between teacher and student exists. It is possible to interfere immediately even if there is no physical presence, overcoming one of the gaps that is present their learning systems.

The objective of this work is to create a system capable of supervising and improving attentiveness of each student, their learning style, as well as their user behavior interaction with the peripheral devices. From there, the system should be able to suggest the teacher the most effective method of learning for each student, at a given moment, to minimize the lack of attention.

The long-term goal is to guide teachers with valuable information about the profile and the status of students, allowing the teacher to better manage the teaching methodologies for obtaining better results.

\subsection{DYNAMIC FRAMEWORK OF ATTENTION ANALYSIS}

As explained before, this project focuses on creating a multi-modal distributed system, in which the software correlates data collected through different computer devices involving biometric and behavioral analysis to assess the attentiveness of an individual. After the system determines the attention condition of an user by requiring the analysis of four types of information: mouse behavior, keystroke behavior, worktask, and decision-making patterns. This system was called Seamless Supervising Assistant (3S's).

One of the challenges of this approach has to do with data manipulation. The growth of the large amount of data, its variety, and the speed which the data emerge bring challenges to processing, storage, and analysis. To solve this situation is presented a framework that accommodates the data pipeline of data generated by human computer, providing real-time data analysis on behavioral biometrics.

As it is shown in Figure 4.3 the framework of 3S's is divided into three main parts: the lowest level with the devices that generate data; the intermediate level where the cloud is located; and the highest level, the client system. 
At the lower level, the system's as a logger application that runs in the background, collecting the necessary events. The devices that generate the raw data (e.g. soft sensors) describe the student's interaction with both mouse and keyboard. There are soft sensors that use information available from other measurements and process parameters to calculate an estimate of the quantity of raw data. The raw data generated is stored locally until it synchronises with the web server in the cloud at regular intervals. In this layer, each event is encoded with the corresponding required information (i.e. timestamp, coordinates, type of click, key pressed, etc.).

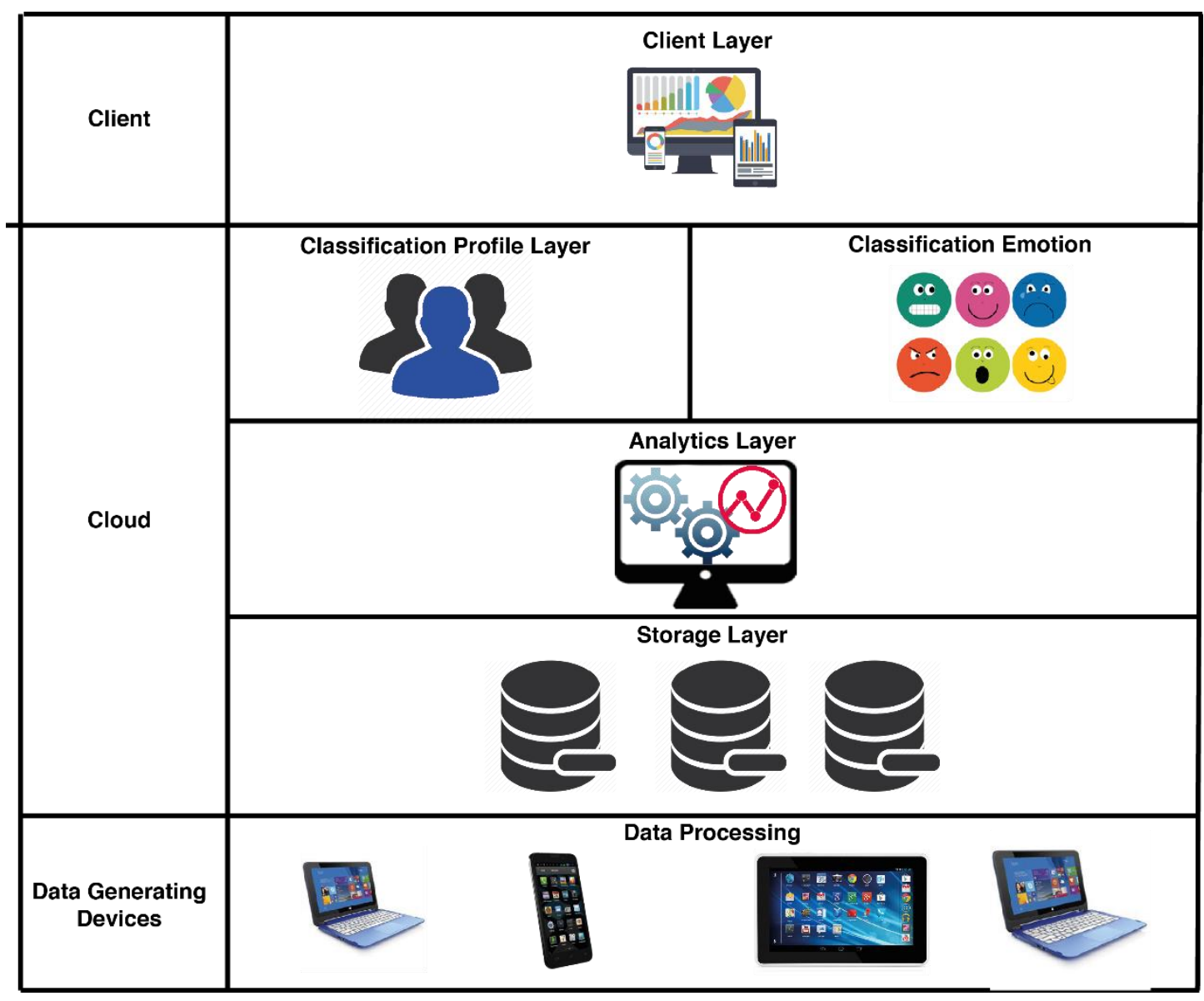

Figure 4.3 - Dynamic Framework of attention analysis.

The intermediate level is subdivided into four layers: the storage layer, the analytic layer, the classification profile layer, and the emotion classification layer.

In the storage layer, a MongoDB database stores the data received from users when it is synchronised. The MongoDB database is a cross-platform, document-oriented database that provides, high performance, high availability, and 
easy scalability. It is also works on concepts of collection and document. MongoDB, in addition, to be a data storage engine, also provides native data processing tools such as Map Reduce and the Aggregate Pipeline. Both procedures can operate in a shared collection (partitioned on several machines with horizontal scaling).

In the analytical layer, some processes were developed that aim to prepare the received data, such as removing outlines (for example, the backspace key being continuously pressed to delete a character set is not a regular key press), so that this data is evaluated according to the presented metrics. In addition, the system receives this information in real time and calculates, at regular intervals, the values of behavioral biometrics and the estimation of the general level of attention of each student. These are powerful tools for performing analytic and statistical analysis in real-time, which is useful for ad-hoc querying, pre-aggregated reports, and more. MongoDB provides a large set of aggregation operations that process data records and return corrected results, allowing the use of these operations in the data layer to simplify application code and limit resource requirements.

In the classification profile layer, all user's indicators are interpreted. Based on the preprocessed data and on the construction of the metadata that will support decision making, the system will classify the user's profile. When the system presents a large enough set of study cases, it is possible to make classifications accurately. The classifier, in real time, will classify the data received from the different levels of attention, creating the learning profile of each student. With these results, it is possible to obtain a profile of the learning style.

The classification emotion layer has all user's emotion data and the construction of metadata that will support decision making. The system will sort the user's emotional profile and, when the system has a high set of data, it makes it possible to make ratings accurately. Note that mouse movements and keyboard usage patterns also help predict the mood of the user.

Finally, a web application is available at the client layer where students can visualize the tasks they must complete. Moreover, for the managers (teacher), the user's attention information is displayed at the client layer. The graphical user interface present at this layer consists of a module that allows the creation of 
graphics (CHART) and allows the creation of virtual teams (ROOM or Classes) so that the administrator can intuitively visualize the student's behavior.

\subsection{S'S - SEAMLESS SUPERVISING ASSISTANT}

Based on the previous section (4.2), in this section it will be explained the architecture of 3S's more detailed.

As explained before, this project focuses on creating a multi-model monitoring distributed system of attention assessment, in which the software correlates data collected through different computer devices involving biometric and behavioural analysis to assess the attention level of an individual. To determine the attention level condition of an individual, it requires the analysis of three types of information: mouse behavior, keyboard behavior, and work-related task. As such, the work can be decomposed into the following components:

$>$ MouseDynamics: A framework that includes the acquisition and classification of mouse input data based on biometric behavior.

$>$ KeyboardDynamics: A framework that includes the acquisition and classification of keyboard input data based on biometric behavior.

$>$ Work-related task: A framework that includes the acquisition and classification of work-related tasks based on the user applications used.

Since the study of behavioral features is mostly related to the individual's conduct habits, the calculation of attention level considers the individual's ID, actions of mouse and keyboard, and the application that the user used, which are monitored and acquired by the computation system (Zhou, et al., 2015).

After pre-processing it, data are stored in a MongoDB database for future data mining. Through these techniques, it is possible to (1) study their behavioral patterns, so that we can obtain their behavioral pattern and their influence in the learning process; (2) study the relations between the affective reactions of the individual and their behavioral patterns in the cyberspace, so that we can make an evaluation of emotions and their influence in the learning process (Dai, Duch, Abdullah, Xu, \& Chen, 2015). 
Finally, it is possible to obtain the level of attention of each student in each situation, the user learning style, and predicting features in news situations. The explained architecture is shown in Figure 4.4. This information is displayed at the client layer, in a graphics visualization or in a table visualization so that the administrator (teacher) can intuitively visualize the student's behavior.

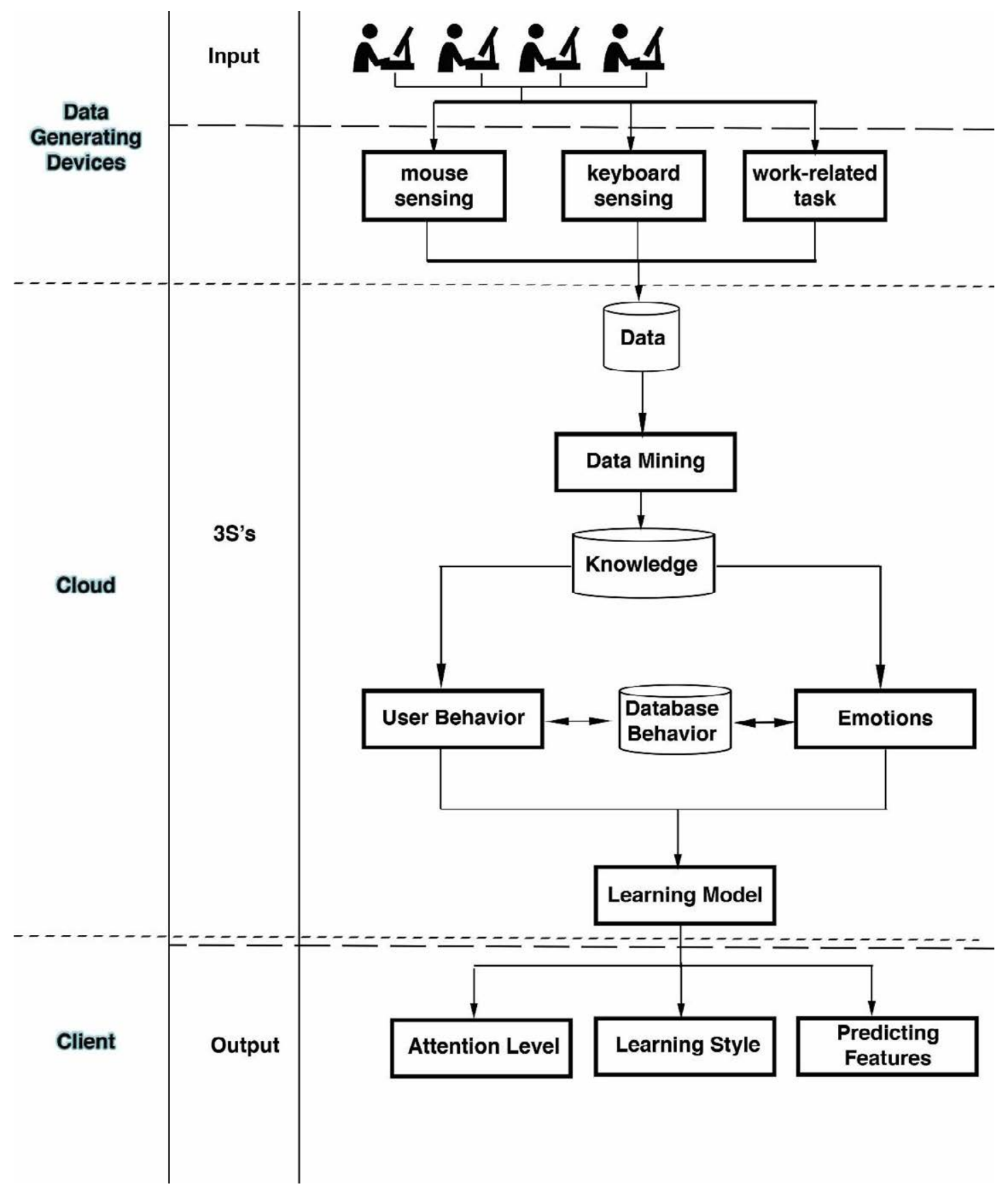

Figure 4.4 - Proposed framework 


\subsubsection{FEATURES EXTRACTION}

The process of feature extraction starts with the acquisition of interaction events, which is carried out by a specifically developed application that is installed in each computer or laptops. Logger applications that run in the background, collecting the necessary events and require no interaction by the user. It is thus non-intrusive (Carneiro, Pimenta, Gonçalves, Neves, \& Novais, 2015). The soft sensors used information available from other measurements and process parameters to calculate an estimate of the quantity of interest. Figure 4.5 presented an example of a behavioural biometrics of mouse and keyboard user.

\section{Keyboard Dynamics KD}
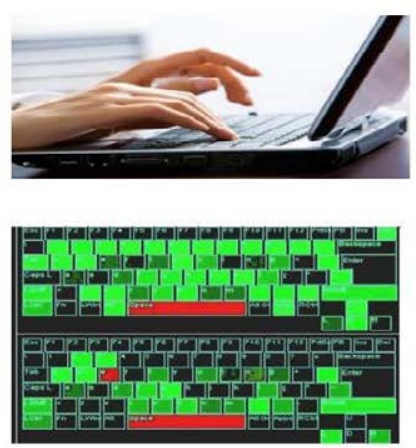
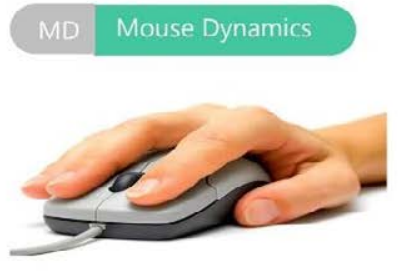

Behavioral Biometrics
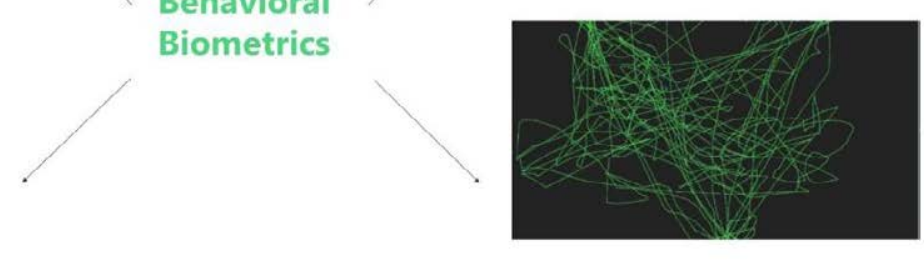

Figure 4.5 - Behavioral Biometrics.

The data collected by the data collection application results in a record of the set of events caused by the interaction of the user with the mouse and the keyboard. This information alone is useless as it does not allow the extraction of relevant and interpretive information. It is then necessary to process the information related to the events recorded and to transform this same information into the characteristics presented to study so that it can be withdrawn and analyzed the information coming from the record of events caused by the mouse and keyboard interaction. In order to process the events registered, an application was used that receives as input the recorded events and transforms this dataset into parameters that will be evaluated by the defined metrics, which are the result of the application as output.

The following events are acquired by the application and sent to the server for processing (Novais \& Carneiro, 2016): 
MOV, timestamp, posX, posY: an event describing the movement of the mouse, in a given time, to coordinates (posX, pos $Y$ ) in the screen;

> MOUSE DOWN, timestamp, [Left | Right], posX, posY: this event describes the first half of a click (when the mouse button is pressed down), in a given time. It also describes which of the buttons was pressed (left or right) and the position of the mouse in that instant;

$>$ MOUSEUP, timestamp, [Left|Right], posX, posY: an event similar to the previous one but describing the second part of the click, when the mouse button is released;

> MOUSEWHEEL, timestamp, dif: this event describes a mouse wheel scroll dif, in a given time;

$>$ KEY DOWN, timestamp, key: this event identifies a given key from the keyboard being pressed down, at a given time;

$>$ KEY UP, timestamp, key: this event describes the release of a given key from the keyboard, in a given time.

The individual logs created by the application for each user are processed to compile information that can characterize the user's behavior during their interaction with the computer. This subsection details the resources extracted from these interaction event logs. Table 4.1 presented the general information of the dataset collect from the user's behavioural biometrics.

Table 4.1 - General view of dataset of behavioural biometrics.

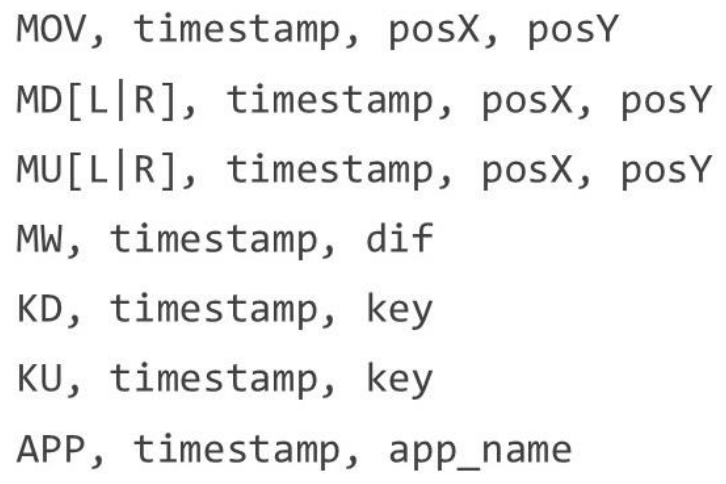

The following example shown in Table 4.2 depicts a brief log that starts with some mouse movement (first two lines), contains a click with a little drag (lines 3-5) and ends with some more movement (last two lines). 


\subsubsection{ANALYTICS}

MouseDynamics data-log output analyses the individual's mouse behavior and calculates his/her behavioral biometrics. These features aim at quantifying the individual mouse performance. Taking as an example the movement of the mouse, one never moves it in a straight line between two points, there is always some degree of curve. The larger the curve, the less efficient is the movement is (Carneiro, Pimenta, Gonçalves, Neves, \& Novais, 2015), (Carneiro, Novais, Pêgo, Sousa, \& Neves, 2015). The following example is shown in Table 4.2.

Table 4.2 - mouse movement example.

MOV,635296941683402953,451,195

MOV,635296941684123025,451,197

MOUSE_DOWN,635296941684443057, Left ,451,199

MOV,635296941685273140,452,200

MOUSE_UP,635296941685283141, Left ,452,200

MOV,635296941685723185,452,203

MOV,635296941685803193,454,205

KeyboardDynamics data-log output analyses the individual's keyboard behavior and calculates his/her behavioral biometrics. These features aim at quantifying the individual keyboard performance. Taking as an example the keys pressed, the writing velocity of each person is different and in a different time, people write at different velocity. In table 4.1 , it is showing how the information of the keyboard from a dataset can be shown.

Work-related task analyses the individual's using tasks and calculates his/her time spend in each task. These features aim at quantifying the individual work-task performance.

\subsubsection{Analytics Data from Mouse and Keyboard Interaction}

It is important to note that these features are intended to quantify user performance. Taking the mouse as an example, its movement between two points is never straight, as there is always some deviation. The greater this deviation, the less efficient is the movement. One of the interesting properties of the features described 
in this section is that, except for mouse velocity and acceleration (as detailed below), an increasing deviation value denotes a decreasing work. These relationships with performance have been established in previously conducted research studies (Carneiro, Pimenta, Gonçalves, Neves, \& Novais, 2015).

In Figure 4.6 it is presented the information obtained in analytics level that it is used from the interaction with the mouse and keyboard.

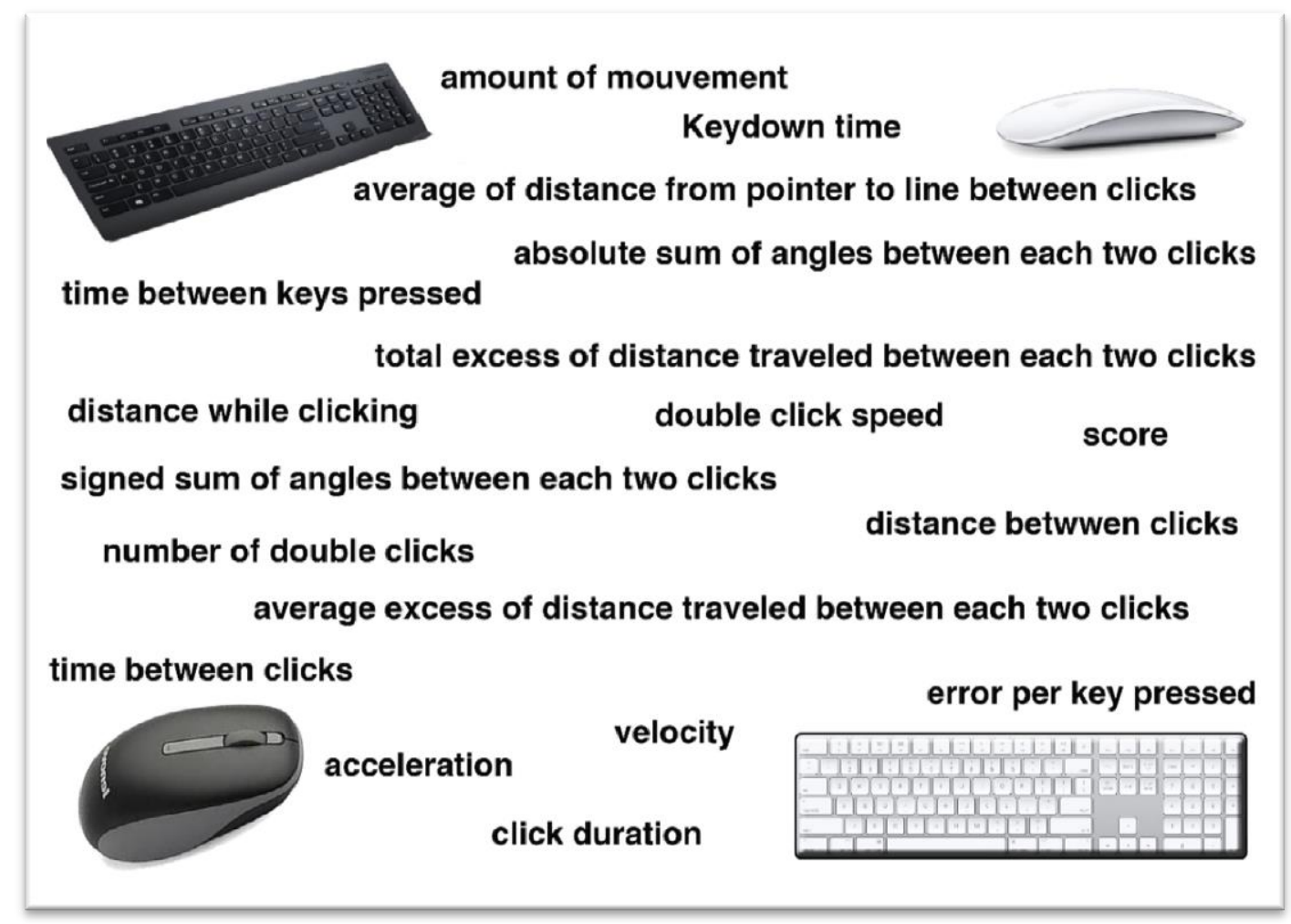

Figure 4.6 - data result of mouse and keyboard interaction.

Regarding the behavioral biometrics of the following 15 features considered, 12 are extracted from the mouse and 3 are extracted from the keyboard (Durães D. , Carneiro, Bajo, \& Novais, 2016):

$>$ Mouse Velocity (MV): The distance travelled by the mouse (in pixels) over the time (in pixels/milliseconds).

$>$ Mouse Acceleration (MA): The velocity of the mouse (in pixels/milliseconds) over the time (in pixels/milliseconds ${ }^{2}$ ). 
> Click Duration (CD): Measures the time span between two consecutive mouse clicks. The longer the clicks, the less efficient the interaction is (in milliseconds).

> Time Between Clicks (TBC): The time span between two consecutive mouse clicks (in milliseconds).

> Time Double Click (TDC): The time span between two consecutive MOUSE UP and MOUSE DOWN events when smaller than 200 ms i.e. the duration of a double click.

$>$ Distance Between Clicks (DBC): Represents the total distance travelled by the mouse between two consecutive mouse clicks (in pixels).

$>$ Excess of Distance (ED): This feature measures the excess of distance that the mouse travelled between each two consecutive MOUSE UP and MOUSE DOWN events (in pixels).

$>$ Absolute Sum of Angles (ASA): This feature seeks to find how much the mouse turned, independently of the direction to which it turned (in degree units).

$>$ Signed Sum of Angles (SSA): This feature is very similar to the previously mentioned one, with the exception that it measures to which side the mouse turns more (in degrees).

$>$ Average Distance of the Mouse to the Straight Line (ADMSL): This feature quantifies the average sum of the successive distances of the mouse to the straight line defined by two consecutive clicks (in pixels).

$>$ Average Excess of Distance Between Clicks (AED): This feature measures the average excess of distance that the mouse travelled between each two consecutive mouse clicks (in pixels).

$>$ Distance of the Mouse to the Straight Line (DMSL): This feature quantifies the sum of the successive distances of the mouse to the straight line defined by two consecutive clicks (in pixels).

$>$ Key Down Time (KDT): The time span between two consecutive KEY DOWN and KEY UP events, i.e. for how long did the user press a given key (in milliseconds).

$>$ Time Between Keys (TBK): The time span between two consecutive KEY UP and KEY DOWN events, i.e. how long did it take the user to press another key after releasing the previous one (in milliseconds). 
$>$ Writing Velocity (WV): The time span between two consecutive KEY UP and KEY DOWN events, i.e. how long did it take the user to press another key (keys per minute).

Given the complexity of some features, Figure 4.7 presents a brief explanation of some metrics with graphic examples.

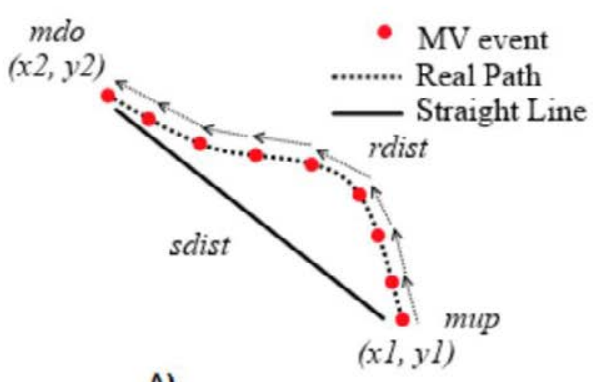

A)

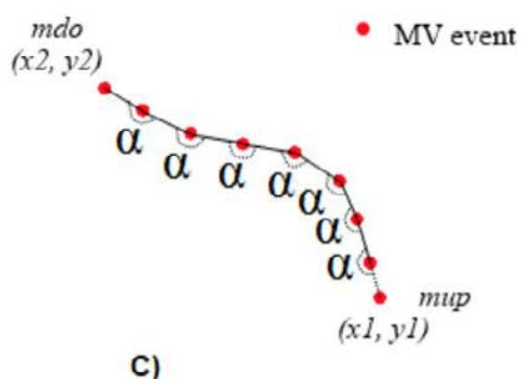

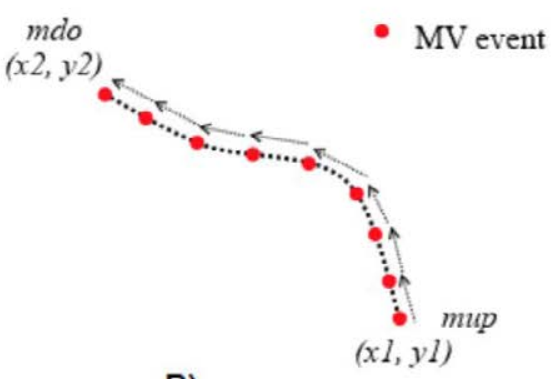

B)

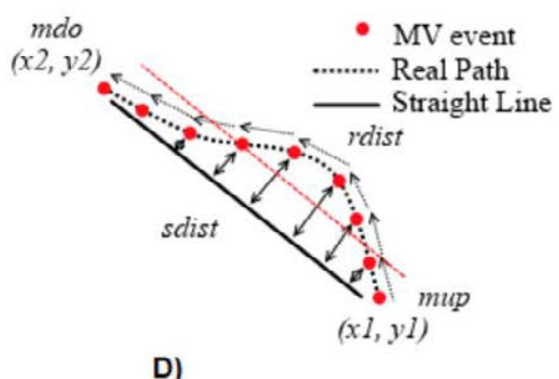

D)

Figure 4.7 - (A) A series of mouse movements events (MV), between two consecutive clicks of the mouse. The difference between the shortest distance (sdist) and distance actually travelled by the mouse (rdist) is depicted; (B) The real distance travelled by the mouse between each two consecutive clicks is giving by summing the distances between each two consecutive MV events; (C) The sum of the angles of the mouse's movement is given by summing all the angles between each two consecutive movement vectors; (D) The average distance at which the mouse is from the shortest line between two clicks is depicted by the straight dashed line (Carneiro \& Novais, 2017).

\subsubsection{Analytics Data from Work-related Task}

To obtain the time spent in a work-related task it is necessary to know the time that each user spend in each task. However, at the beginning, it is necessary that the instructor (teacher) defines the task-related applications that the students will use during the class. The team administrator uses a graphical interface to set rules such as "contains youtube" or "Contains photoshop" (see Figure 4.8) who are then translated into regular expressions that are used by the algorithm to determine which applications are and are not work-related. In this sense, it is necessary to measure 
the amount of time in each interval, that the student spent interacting with work taskrelated applications. By default, applications that are not considered task-related are marked as "others" and count negatively towards the quantification of attention.

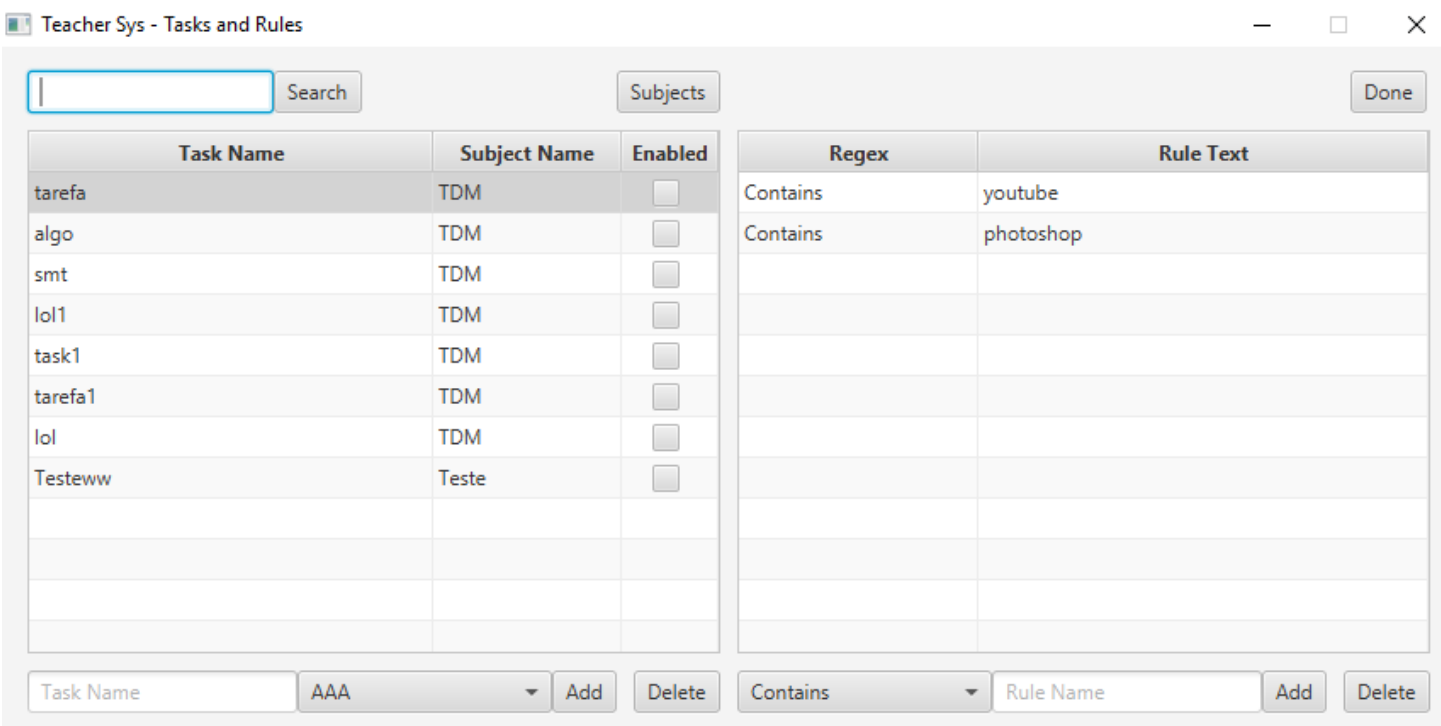

Figure 4.8 - Client Visualization selecting work task-related.

When the student uses an application that does not match any of the known rules for a specific task, the application name is saved so that the teacher can later decide if a new rule should be created for it. An example of the output of this process is depicted in Figure 4.9.

\begin{tabular}{|c|c|c|}
\hline aplicaÃ§oes - Microsoft Word & Mon 7 Dec 2015 08:58:32 GMT & $3.886 \mathrm{~s}$ \\
\hline MEO Cloud - Home - Google Chrome & Mon 7 Dec 2015 08:58:33 GMT & $1.088 \mathrm{~s}$ \\
\hline Explorador do Windows & 进 Mon 7 Dec 2015 08:58:35 GMT & $2.01 \mathrm{~s}$ \\
\hline Iniciar & 湎 Mon 7 Dec 2015 08:58:51 GMT & $15.441 \mathrm{~s}$ \\
\hline MEO Cloud - Home - Google Chrome & Mon 7 Dec 2015 09:01:46 GMT & $2.92015 \mathrm{~min}$ \\
\hline Code.org - Star Wars: Construindo uma Gal⿵̃ ${ }_{i} x i a$ com $C \tilde{A}^{3}$ digo - Google Chrome & Mon 7 Dec 2015 09:03:24 GMT & $1.63702 \mathrm{~min}$ \\
\hline Microsoft Forefront Client Security & 湽 Mon 7 Dec 2015 09:06:49 GMT & $3.40668 \mathrm{~min}$ \\
\hline 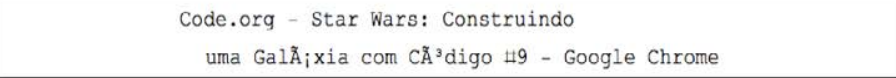 & 苗 Mon 7 Dec 2015 09:07:02 GMT & $13.398 \mathrm{~s}$ \\
\hline $\begin{array}{l}\text { Code.org - Star Wars: Construindo } \\
\text { uma Gal } \AA_{i x i a} \text { com } C \AA^{3} \text { digo } \sharp 9 \text { - Google Chrome }\end{array}$ & Mon 7 Dec 2015 09:07:47 GMT & $45.099 \mathrm{~s}$ \\
\hline
\end{tabular}

Figure 4.9 - Sequence of applications used by a specific student, with the date in which the student switched to other application and the time spent interacting with it. 


\subsubsection{USER BEHAVIOR}

Based on the framework described in section 3.2, a set of behavioral features are monitored and preprocessed by the proposed system. Through these features, it enables the development of a classification model capable of determining the task at hand executed by the user, given the influence of the user's biometric behaviors. For this study, keystroke dynamics, mouse dynamics, attention performance metrics, and type of learning style were selected to this end.

As described in subsection 4.3.2.1 mouse dynamics describe an individual's behavior with a computer-based pointing device (e.g. mouse). Recently, mouse dynamics have been proposed as a behavioral biometric, under the premise that mouse behavior is relatively unique among different people. An example is presented in Figure 4.10.
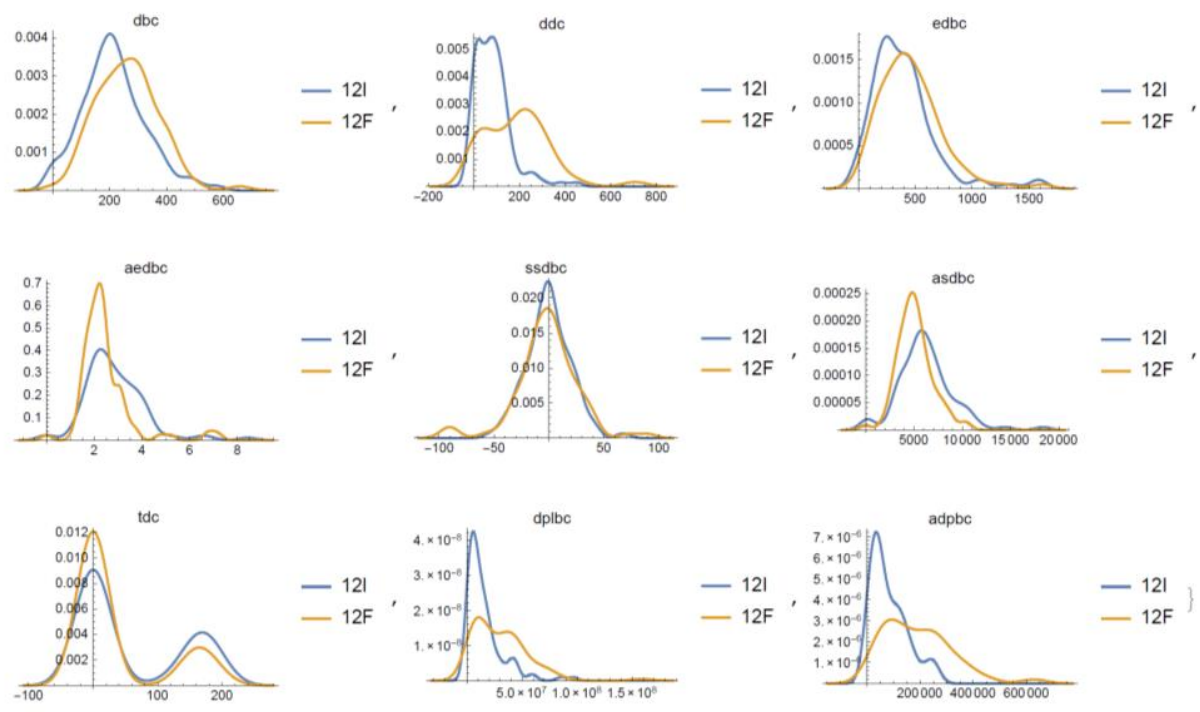

Figure 4.10 - Mouse Behavior Biometrics of two class.

Another way of monitoring the user's behavior in human-computer interaction $(\mathrm{HCl})$ is based on keystroke analysis (see in Figure 4.11). The work task-related is obtained by recording the timestamp in which each student switched to a specific application, by recording the user's ID, timestamp and application name (see Figure 4.13). In subsection 4.3.5 and 4.3.6 it is described the attention performance metrics and learning style classification. 


\section{TBK}

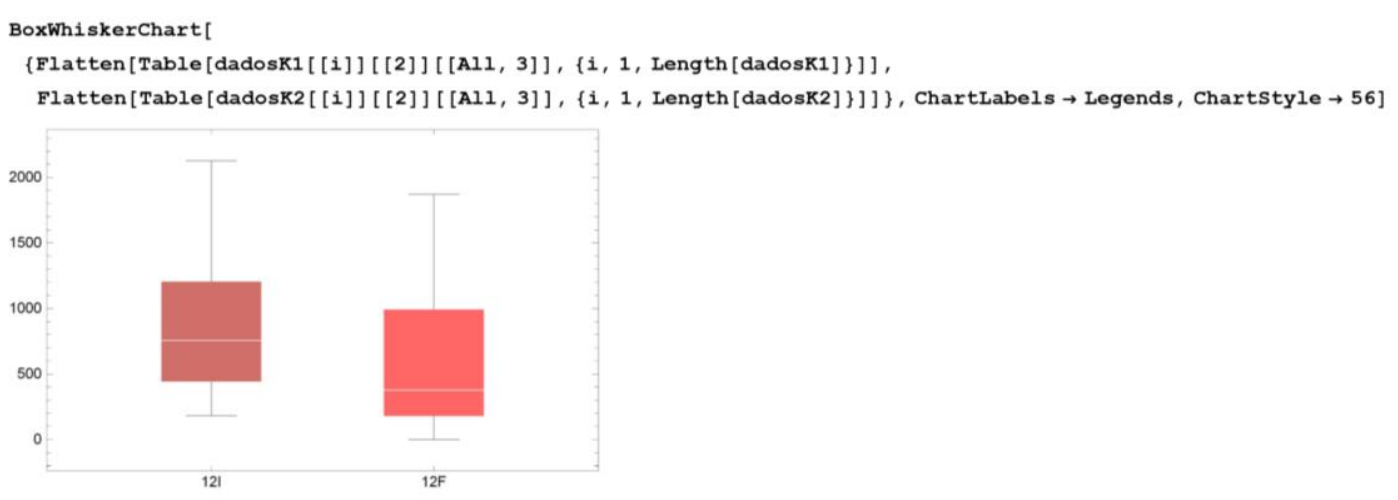

Figure 4.11 - Example of a Keyboard TBK of two classes.

\subsubsection{EMOTIONS}

The interest of researchers in the field of $\mathrm{HCl}$ and to analyze if the effects of stress, affective states, fatigue and other factors on the individual's interaction patterns with the technological devices is increased. As when considering interactions between people, interactions between people and technological devices also have two channels: one transmits explicit messages (i.e. the actions we perform on the computer) while the other transmits implicit messages (i.e. how we do it). As research has been demonstrating, we perform actions differently according to our state. The inclusion of this type of information in $\mathrm{HCl}$ projects of the next generation is seen by many experts as the way to the production of true systems of human consciousness, able to understand and adapt to the state of the user at any moment (Pantic \& Rothkrantz, 2003).

The motions of the user's hand and by extension the movements of the computer mouse have a direct relation with the psychological - sentimental condition of the user. To be more specific, the way by which the mouse is moved (orbit, speed, intervals of immobility, direction) can demonstrate the user's condition (Carneiro \& Novais, 2017), (Durães D. , Carneiro, Bajo, \& Novais, 2016).

The way a user types may indicate his/her state of mind. Pressing rapidly the keyboard could mean an altered state, anger or stress for instance, while taking too much time may mean sadness or fatigue. Keystroke dynamics, which measure an 
individual's typing rhythms, have been the subject of considerable research over the past few decades and their use for emotion recognition has shown promising results. (Rodrigues, Riverola, \& Novais, 2012), (Carneiro, Pimenta, Gonçalves, Neves, \& Novais, 2015).

For each student, the collected data is aggregated and summarized at 5minute intervals. The average value of the feature in the interval is used. Figure 4.12 depicts the type of information that these features provide. It shows the evolution of the performance of a specific student during an exam through two features: Click Duration and Mouse Velocity. The duration of each click decreases until roughly the middle of the exam and then increases up to a global maximum. The velocity of the mouse increases until approximately the same point in time and then it starts decreasing. Both features point out an initial improvement of performance (faster clicks and increasing mouse velocity), followed by a degrading. Figure 4.12 actually reveals a classical effect of stress: performance tends to improve for some time after the beginning of the stressor stimulus (eustress), with a drop off in performance after some time performing above average (distress). These features allow for an individualized view on how stress affects each particular student, potentially devising each one's breaking points, behavior or overall performance under stress.
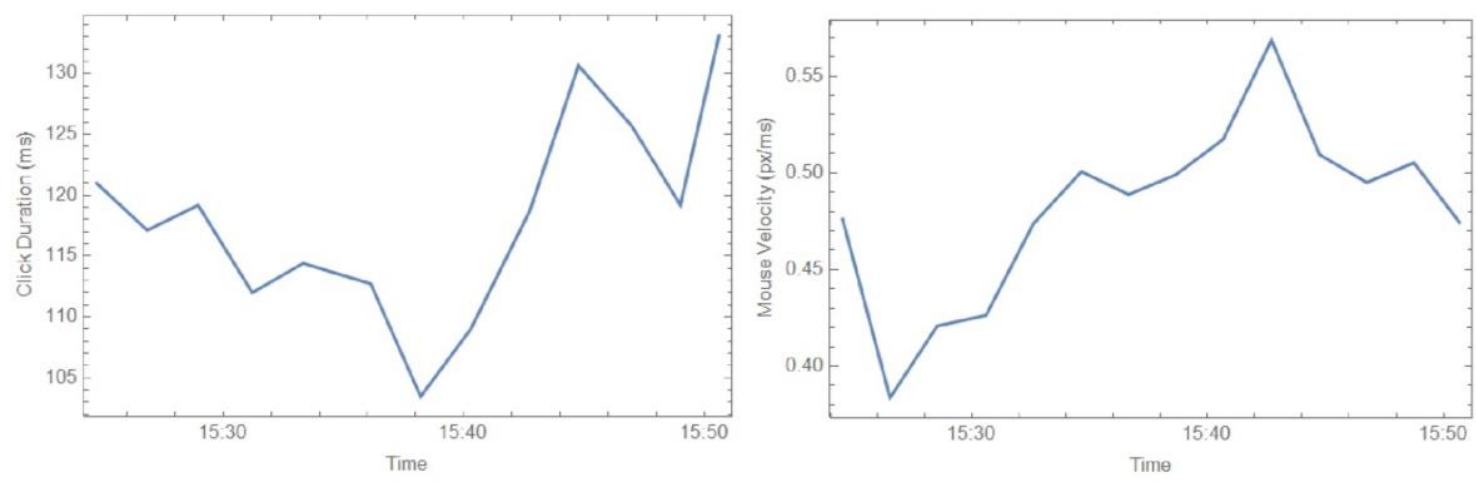

Figure 4.12 - Real-time performance: evolution of one student's interaction performance during the exam. Left: Click Duration. Right: Mouse Velocity.

\subsubsection{CLASSIFICATION OF ATTENTION LEVEL}

The goal of this feature is to classify the level of the user's attention when perform a task at the computer. The performance is achieved with the user's 
behavior using the mouse and the keyboard and the several applications that were accessed.

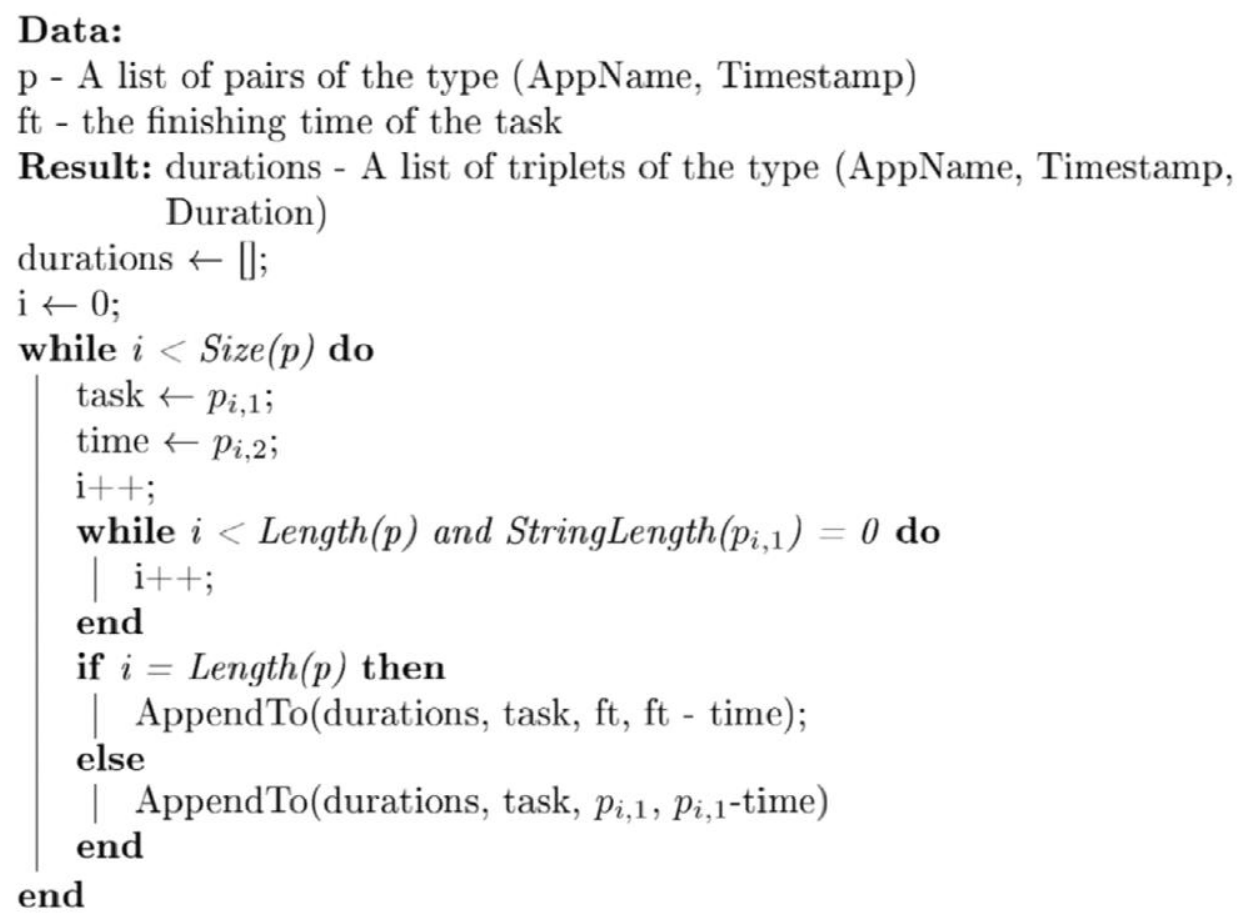

Algorithm 4.1 - Creating triplets with the durations and timestamp of each application (Carneiro, Durães, Bajo, \& Novais, 2016).

Algorithm 4.1 specifically, it goes through the list of pairs and computes the time during which each window was active. There are often cases in which the user does not change applications for a large amount of time. In these cases, which are represented by a pair with an empty AppName, the time is added to the last known AppName (since this means that the user is still interacting with it).

First, it is necessary to compute the level of attention of the user (Algorithm, 4.2). To do this we measure the amount of time, in each interval, that the user spends interacting with work-related applications. The algorithm thus needs knowledge about the domain to classify each application as belonging or not to the set of work-related applications. This knowledge is provided by the team administrator and is encoded in the form of regular expressions. The team administrator uses a graphical interface to set up rules such as "starts with Microsoft" 
or "Contains word Adobe", which are then translated to regular expressions that are used by the algorithm to determine which applications are work-related.

Whenever an application that does not match any of the known rules for the specific domain is found, the application name is saved so that the team manager can later decide if a new rule should or should not be created for it.

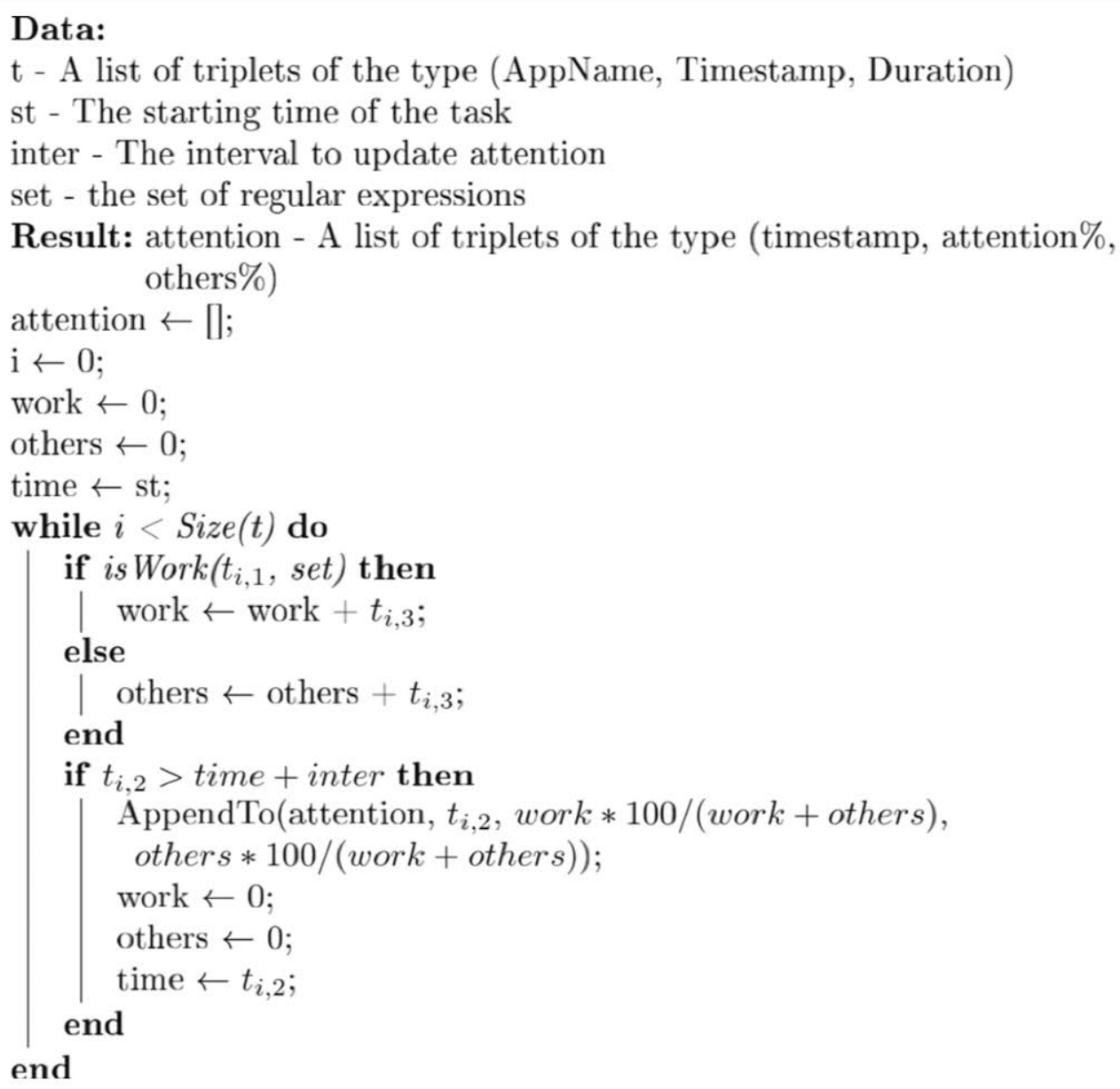

\section{Algorithm 4.2 - Creating triplets at regular intervals with the timestamp and the quantification of} attention (Carneiro, Durães, Bajo, \& Novais, 2016).

By default, applications that are not considered work-related are marked as "others" and count negatively towards the quantification of attention. Attention is calculated at regular intervals, as configured by the team manager (e.g. five minutes). The output of the algorithm can be visualized in Figure 4.13. 
Secondly, we calculated the percentage of the interaction of the mouse, which is the average number of times that the mouse was pressed in the class considering the total of interactions with the mouse in the class. Similarly, we calculated the percentage of the interaction of the keyboard, which is the average number of times that the keys were pressed in the class considering the total of interactions with the keyboard in the class.

\begin{tabular}{|c|c|c|}
\hline Date & $\%$ Work & $\%$ Others \\
\hline 㠿 Thu 3 Mar 2016 14:49:43 GMT & 88.8875 & 11.1125 \\
\hline 眥 Thu 3 Mar 2016 14:54:49 GMT & 43.9485 & 56.0515 \\
\hline 进 Thu 3 Mar 2016 14:59:49 GMT & 86.9204 & 13.0796 \\
\hline 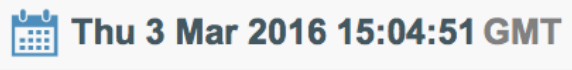 & 74.5224 & 25.4776 \\
\hline 进 Thu 3 Mar 2016 15:10:25 GMT & 99.5259 & 0.47408 \\
\hline 㢤 Thu 3 Mar 2016 15:15:28 GMT & 92.614 & 7.38601 \\
\hline 进 Thu 3 Mar 2016 15:20:41 GMT & 99.3591 & 0.640935 \\
\hline 进 Thu 3 Mar 2016 15:25:55 GMT & 100 & 0 . \\
\hline 㖆 Thu 3 Mar 2016 15:30:57 GMT & 96.6265 & 3.37347 \\
\hline 匊 Thu 3 Mar 2016 15:38:01 GMT & 26.6544 & 73.3456 \\
\hline 氟 Thu 3 Mar 2016 15:43:06 GMT & 96.4635 & 3.53646 \\
\hline 进 Thu 3 Mar 2016 15:49:05 GMT & 98.7674 & 1.23265 \\
\hline 觜 Thu 3 Mar 2016 15:52:44 GMT & 97.0061 & 2.9939 \\
\hline
\end{tabular}

Figure 4.13 - Detail of evaluation of attention for a specific student (Durães D. , Carneiro, Bajo, \& Novais, 2016).

On the order hand, we obtained the amount of time that each student spent at the computer (Task Duration) as well as the amount (and percentage) of time that each student devoted to work and to other activities in the lesson as explained in 
Carneiro, Durães, Bajo, and Novais (Carneiro, Durães, Bajo, \& Novais, 2016). However, the time that the student spent in the task-related does not indicate the level of attention in some cases, because in some situations the user might have opened the task-related and during that time he/she did not interact with the computer. In this case, if the level of attention was measured only by the taskrelated, the student has $100 \%$ of attention and in reality, his/her attention level should be $0 \%$. For these situations, it is necessary to analyse the amount of interaction with the mouse and the keyboard and cross these data.

The next step is to calculate the percentage of usage of the mouse for each user. The formula to calculate this value is:

$$
I M=\frac{\sum M d}{M a x M d} \quad \text { (Equation 1) }
$$

Where:

IM: is interaction of the mouse;

Md: is the number of the times that the mouse was pressed by a user;

MAX Md: is the higher score of usage of the mouse down in the class.

Similarly, it is necessary to calculate the percentage of usage of the keyboard for each user, the formula for this value is:

$$
I K=\frac{\sum K d}{\operatorname{Max} K d}
$$

\section{(Equation 2)}

Where:

IK: is Interaction of the keyboard;

$\mathrm{Kd}$ : is the number of the time that one key was pressed by a user;

Max Kd: is the higher score of usage of the Keyboard in the class.

Finally, in order to obtain the level of attention it is necessary to combine the results of the interaction of the behavior biometrics with the results showed in the time that each student spent in the task-related. This level of attention is the relative attention of each student, as this level of attention is compared with the other user of the class. Equation 3 provides the results for each user, presented in Table 3. The Equation 3 that calculated these values is:

$$
\text { Rel. } A t=\left(\left(P e r c M * W o r k \_r e l\right)+\left(P e r c K * W o r k \_r e l\right)\right)(\text { Equation 3) }
$$

Where: 
Rel.At: is Relative attention;

PercM: is the percentage of utilization of the Mouse for a user;

PercK: is the percentage of usage of the Keyboard for a user;

\subsubsection{LEARNING STYLE'S IDENTIFICATION}

The main goal of this task is to identify the user's learning style. This information will give to the people in charge the best approach that they must take to increased performance.

The learning style identification will be influenced by the task type, the level of attention on that task and a qualitative evaluation given by the response to the user's performance. For the task's results being the most correct possible, it is necessary that the data be constantly collected.

After the data from the user is collected, it is built the dataset that will be used on the identification algorithm. With the dataset ready, the system runs the dataset on the algorithm and then analyses the results. After the data is collected in the database, the data is manipulated, and it is built a set of required data to identify the learning style.

The user's attentiveness, the type of task, and the task evaluation will constitute it. That means that all set of data that built the dataset are related to a specific user measure. This is presented in Figure 4.14.

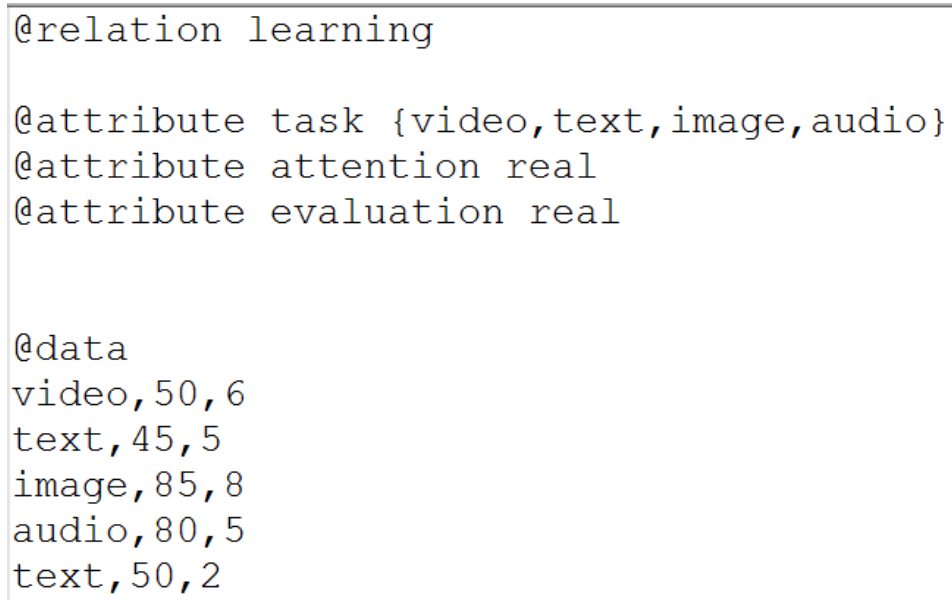

Figure 4.14 - Data set example. 
The property task type is identified by the nominal data video, text, image, and audio. Each number represents a task type and the property attention level is a numeric data and consequently more related to a learning style.

The property attention level is reached by the classification of attention feature. Finally, the property evaluation has to be given by a request for the specific session, where this evaluation drifts between 1 and 10.10 is the higher evaluation and 1 the lower evaluation. This data is given by the responsible and represent the perception that the person has about the user performance.

\subsubsection{K-means Algorithm}

K-means is an unsupervised learning algorithm that can be used to solve clustering problems made by (MacQueen, 1967). Clustering is a data mining technique that divides the data into different groups. The data in each group is related.

The algorithm procedure tries to classify the data over several clusters defined at the beginning of the process. The goal is to define the center point of each cluster and then associate each instance of the dataset to the nearest central point. Then it is recalculated a new cluster central point and the entire instance is again associated with a new central point. When any instance is associated with a new central point the algorithm stops. This is presented in Figure 4.15. 


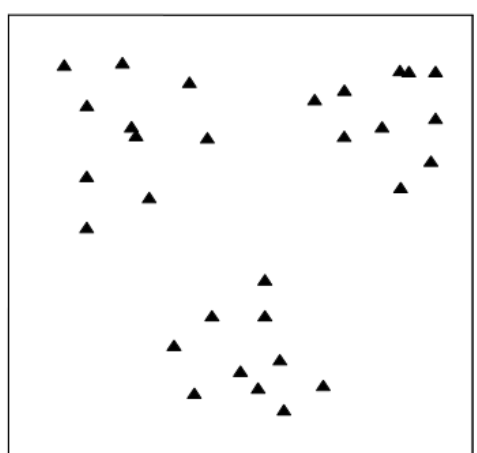

(a) Input data

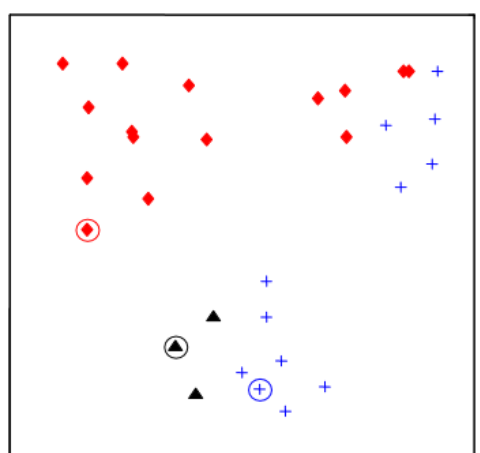

(b) Seed point selection

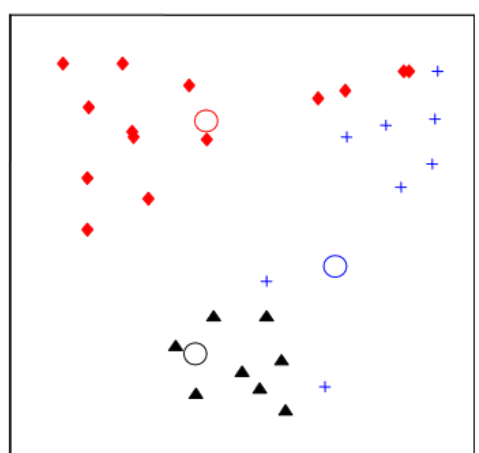

(c) Iteration 2

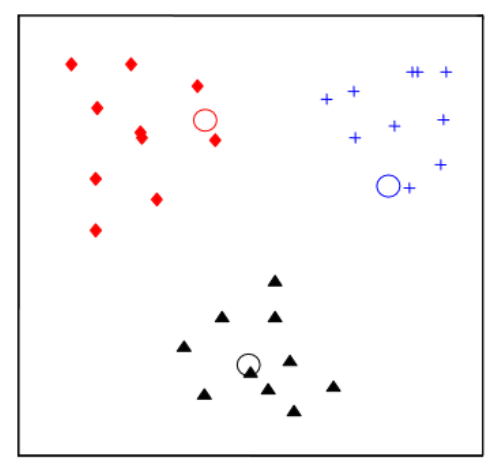

(d) Iteration 3

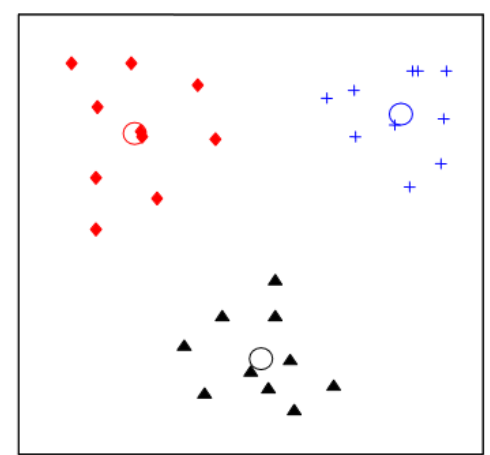

(e) Final clustering

Figure 4.15 - K-means algorithm process example (Anil, 2010)

\subsubsection{Identification Process}

After the creation of the dataset, it will be used a clustering algorithm to group the information on the dataset. The task type related with the Felder-Silverman is presented in Table 4.3. The clustering algorithm that will be used is the k-means algorithm.

Table 4.3 - Relation between type of tasks performed and the learning style theory in a group of users.

\begin{tabular}{l|l}
\hline Task's Type & Learning Style \\
\hline Video & Visual or Verbal \\
Text & Active or Reflective \\
Image & Sequential or Global \\
Audio & Sensing or Intuitive \\
\hline
\end{tabular}




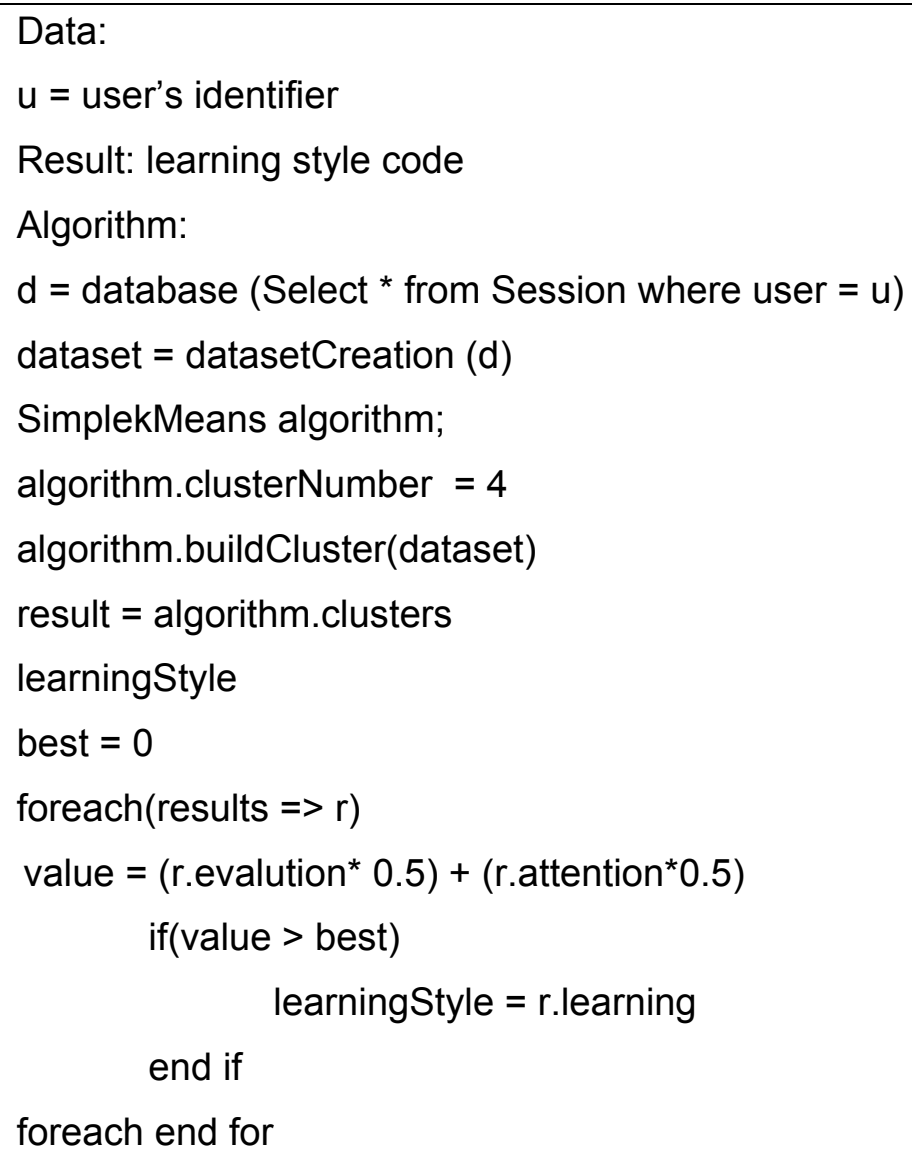

Algorithm 4.3 - Learning Style Process.

Algorithm 4.3. is presented the algorithm used, however, it is necessary to define the number of the clusters that the algorithm needs to look. On this case, it is easy to find the number because the number of the different form of learning was four (video, text, images, and audio), so the algorithm will search for four clusters.

When the clustering algorithm is finished, the results are analysed. The idea was to include the results of the evaluation obtained in each task performed and the level of attention. The weight assigned to each of them was $50 \%$. Finally, the classification of the learning style of each user is obtained through the best classification obtained from the evaluation of each task performed and the level of attention.

\subsubsection{PREDiCTIONS FEATURES}

Based on the biometric features recorded from different soft sensors, the distribtion of each feature (e.g. mean, median, standard deviation, etc.) presented in 
different scales. To solve this problem, it was necessary to apply features scaling (i.e. normalization techniques). In this study, the two methods used were max-min normalization and Z-score normalization. Min-max normalization technique is a normalization strategy which linearly scales a feature value to the range $[0,1]$, based on the minimum and maximum values of the set of observed values. In other words, the minimum value of the feature value is mapped to 0 while the maximum value is mapped to 1. As for Z-score, this technique is a stand-in for the actual measurement, and they represent the distance of a value from the mean measured in standard deviations. This distribution technique is useful when relating different measurement distributions to each acting as a 'common denominator'.

With this, several machine learning categorization methods were used to predict the student's activity, through the analysis of his/her behavior in HMI. Several classifiers were trained and tested in order to determine the most efficient method to categorize the student's activity, where the most applied methods in the scientific literature were taken into account. The set of classification methods trained and tested were: Support Vector Machine, Nearest Neighbor, Naive Bayes, Neural Network and Random Forest (BORRAJO, Baruque, Corchado, Bajo, \& Corchado, 2011), (Bajo, Paz, Rodríguez, \& González, 2011).

As for the validation method, a split validation method was used to determine the classification performance, where $2 / 3$ of the study cases were used for training the classifiers while the remaining $1 / 3$ was used to test it. Table 4.4 presents the set of results for the classifiers performance.

Table 4.4 - Comparative Analysis of Machine Learning Categorisation Performance. Raw MinMax Normalisation Z-score

\begin{tabular}{c|ccc} 
& Raw & Min-Max & Normalisation Z-score \\
\hline Support Vector Machine & $37.5 \%$ & $43.75 \%$ & $37.5 \%$ \\
Nearest Neighbour & $43.75 \%$ & $62.5 \%$ & $43.75 \%$ \\
Naive Bayes & $68.75 \%$ & $75 \%$ & $68.75 \%$ \\
Neural Network & $31.25 \%$ & $56.25 \%$ & $56.25 \%$ \\
Random Forest & $81.25 \%$ & $87.5 \%$ & $87.5 \%$ \\
\hline
\end{tabular}




\subsection{SUMMARY}

The 3S's architecture of the developed system is divided into three main parts: the lowest level with the devices that generate the data; the intermediate level where the cloud is located; and the highest level, the client system.

The data analysis is done in three parts: behavior biometrics, the user's attention classification and user's learning style definition. To classify it, it will be necessary to group the data per student, then analyse if the application used on the specific time matches the defined task rules and measures the amount of time that the user spent on each type of application. The level of attention will be calculated by the percentage of time spent on the application comparatively with the total time and also based on the interaction of the behavioral biometrics. To define the user's learning style, it used a clustering algorithm to find groups of data related by the task type and then with results identify the better learning style for the user.

Several machine learning categorisation methods were used to predict the student's activity, through the analysis of his/her behaviour in HMI (Corchado, Bajo, Paz, \& Rodríguez, 2009), . Some classifiers were trained and tested in order to determine the most efficient method to categorise the student's activity. The set of classification methods trained and tested were used: Support Vector Machine, Nearest Neighbour, Naïve Bayes, Neural Network, and Random Forest.

Based in this chapter, the next chapter presents some case studies performed during this work in order to validate this work. All of these case studies was presented and published in International Conferences and International Journal in the field of Artificial Intelligence. 
"Our intelligence is what makes us human, and AI is an extension of that quality." -

Yann LeCun

\section{ANALYSIS OF PRACTICAL RESULTS}

This chapter presents a group of case studies in which the model 3S's presented in Chapter 4 was used. The main objective of this chapter is to show that the architecture proposed in the previous chapter was validated by case studies. The long-term goal is to empower teachers with valuable information about student status, allowing them to better manage their students' skills and teaching methodologies.

This project was developed in several phases, as well as the validation of the data. Thus, the first validation of data and case studies was related to the monitoring of attention. After obtaining the attention monitoring, the validation of the learning styles was followed, with the application in case studies. Finally, the interaction of the user with the peripheral devices was studied, in order to be able to predict user behaviors.

\subsection{ATtention LEVEL}

The first tests were applied in the monitoring of attention. It was applied in two studies cases in different context. 


\subsubsection{CASE STUDY 1 - NORMAL AND ASSESSMENT LESSON}

We want to determine if the classes with different goals have a significant effect on mouse and keyboard dynamics and how can we estimate attention level.

\subsubsection{Context}

The purpose of this work is to compare a normal and an assessment class at the High School of Caldas das Taipas, Guimarães, Portugal. For this purpose, a group of 13 (10 girls and 3 boys) art students, whose average age is seventeen years old were selected to participate. In different weeks, they have a normal and an assessment lesson, where they have access to an individual computer and three hours to complete a task. The lesson started at 8:30 and finished at 11:00 a.m. in the two lessons.

Students received, at the beginning of the lesson, a document with the goals of the task. The normal and the assessment lessons contained tasks to be completed using Adobe Photoshop.

\subsubsection{Motivation}

An important aspect worth mentioning is that Photoshop requires a precise use of the mouse, which makes it a suitable application to the current study.

The idea is to verify if it is possible monitoring the lessons activity and if it is differences in user interaction pattern in normal and assessment lessons.

\subsubsection{Objectives}

The first objective was compared data from the two lessons and visualized the user's interaction. For second objective we would like to evaluate in this study is the influence of the class in the student, considering the learning techniques and all the surroundings in the environment. The third objective, apply neural networks to classify the classes and estimate a class from the results obtained from a student.

\subsubsection{Results}

In a preliminary analysis of the data, we concluded that there are indeed different interaction patterns depending on the type of lesson analyzed. To conclude this, we looked at the distributions of the data collected and analyzed the statistical 
significance of their differences. To this end, we used the Kruskal-Wallis test. Table 5.1 details the mean value of each feature in each class (evaluation and normal). It also details the $p$-value of the Kruskal-Wallis test.

When data from the two classes is compared, the first conclusion is that the differences observed are statistically significant in nearly all features, with the exception of Time Between Clicks, Distance During Clicks, and Average Distance Point Between Clicks.

Table 5.1 - Results of the Kruskall-Wallis test and mean values for ech class and each feature.

\begin{tabular}{|c|c|c|c|c|}
\hline Feature & Symbol & Significance & Mean Evaluation & Mean Normal \\
\hline Mouse Velocity & $\mathrm{mv}$ & 0,0011 & 0,49 & 0,53 \\
\hline Mouse Aceleration & ma & 0,0010 & 0,54 & 0,57 \\
\hline Click Duration & $\mathrm{cd}$ & 0,0035 & 245,38 & 159,83 \\
\hline Time Between Clicks & tbc & 0,0966 & 1964,19 & 3063,11 \\
\hline Distance Between Click & $\mathrm{dbc}$ & 0,0001 & 150,83 & 206,12 \\
\hline Duration Distance Clicks & ddc & 0,0545 & 143,04 & 143,97 \\
\hline Excess Distance Between Clicks & edbc & 0,0000 & 154,00 & 309,48 \\
\hline Absolute Excess Distance Between Click & aedbc & 0,0000 & 1,54 & 2,05 \\
\hline Absolute Sum Distance Between Clicks & asdbc & 0,0094 & 4006,31 & 5038,24 \\
\hline Distance Point to Line Between Clicks & dplbc & 0,0169 & 21611000,00 & 37026200,00 \\
\hline Absolute Distance Point Between Clicks & adpbc & 0,1361 & 157647,00 & 208223,00 \\
\hline
\end{tabular}

Moreover, mean values of the features are consistently lower in the evaluation class. In most of the features, this indicates an increased performance (e.g. a smaller average distance between clicks means that the student moved the mouse in a more efficient manner). However, in the case of mouse velocity, for example, a smaller velocity could point out a slower, and thus less efficient, movement. In past work we concluded that a slower mouse velocity is indeed necessary for the student to achieve increased accuracy in mouse movement: moving the mouse too fast would make precise movements more difficult to carry out. This is especially true in tasks such as those of this study. A similar trend happens with mouse acceleration and click duration. The remaining features consistently show increased performance in the evaluation class. 
Figure 5.1 shows the distribution of the values for all the users and for each feature. We can see that the results of each feature are very different in the two lessons. The students react in different ways in the assessment and normal lesson. We can also consider that, in general, they are more focused on the assessment lesson because they have a mouse velocity, mouse acceleration, and distance point to line between clicks slower and the click duration was higher.
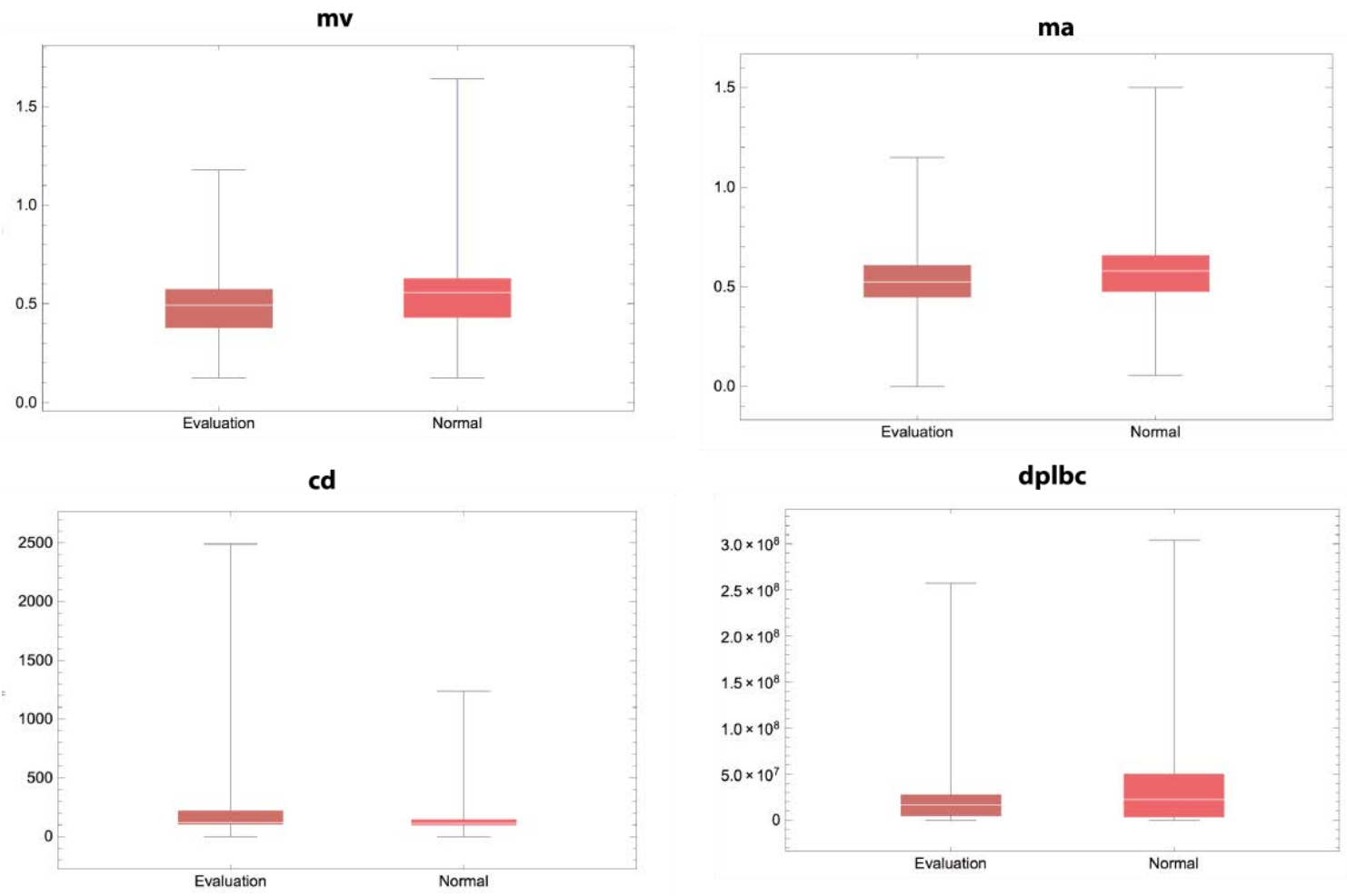

Figure 5.1 - Distributions of the data collected in the two different classes (Evaluation vs. Normal), in four different features: Mouse Velocity, Mouse Acceleration, Click Duration and Distance of the Pointer do the Line Between Clicks.

The selected of features characterize several aspects of interaction on normal and assessment lesson. However, this doesn't mean that they are all affected equally or that they are all affected at all when they have an assessment.

A curious property of these features comparing the normal and the assessment lesson was that the mouse velocity and the mouse acceleration are lower, and the click duration is higher in the assessment lesson. Figure 5.2 compares the histograms of $\mathrm{mv}$, ma, and $\mathrm{cd}$ and evidences the differences between the two classes. 

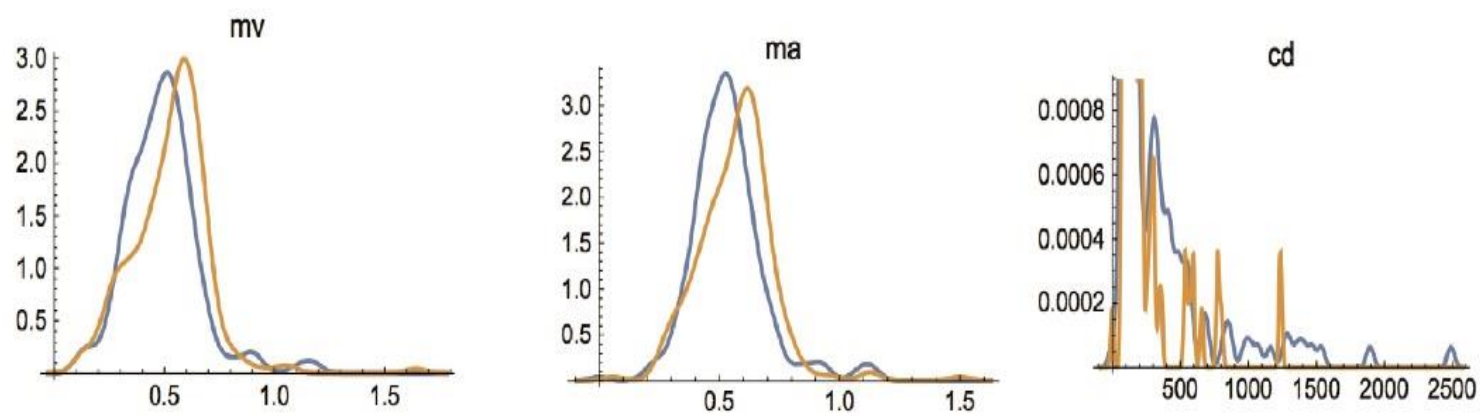

- Evaluation - Normal

Figure 5.2 - Comparing mv, ma, and cd during the lessons.

From these studies we conclude that the most significantly affected features are $\mathrm{mv}, \mathrm{ma}, \mathrm{cd}$, tbc, dbc, ddc, edbc, ssdbc, and dplbc. In all these features almost, all students show statistically significant differences when comparing the two lessons.

Table 5.2 details the results of this quantification. Students have a clearly different attitude in the two lessons: when being evaluated, they spend more time interacting with task-related applications, a sign that they are more focused on the task. The results thus point out, not only, that it is possible to quantify student attentiveness in a non-intrusive way, but also that attention is higher when under evaluation. This was an expected conclusion, but it nonetheless validates the proposed approach. We also analysed the correlation between the level of attentiveness and the score of the students in the task. There is a weak positive correlation (0.41) between the two variables. We believe that this value is not higher for two reasons: (1) this class is mostly composed by "excellent" students and (2) the task was of average difficulty. Thus, there were not many differences in the scores.

After studying the various classification techniques applied in learning systems, this part discusses an ensemble method proposed that combines the predicted outputs from two different classifiers, namely MLP, the traditional classifier and SVM, to obtain better classification results.

The data is supplied to the MLP and SVM classifiers respectively and then the results from both the classifications are combined to give an improved prediction of the data classification. Initially, we proposed an approach based on combining the outputs of two or more experts (MLP and SVM). 
Table 5.2 - Total time (seconds) devoted to the task and percentage of total time devoted to the task, while being assessed and while in a normal class, for each student.

\begin{tabular}{|c|c|c|c|c|}
\hline \multirow{2}{*}{ Student } & \multicolumn{2}{|c|}{ Ass ess ment } & \multicolumn{2}{c|}{ Normal } \\
\cline { 2 - 5 } & Total Time (s) & \% Time & Total Time (s) & \% Time \\
\hline T7110001 & 3156,22 & $67 \%$ & 690,20 & $31 \%$ \\
\hline T7110003 & 2416,01 & $55 \%$ & 1253,07 & $32 \%$ \\
\hline T7110005 & 2475,14 & $63 \%$ & 1807,26 & $40 \%$ \\
\hline T7110006 & 1571,11 & $36 \%$ & 494,07 & $19 \%$ \\
\hline T7110007 & 3177,28 & $53 \%$ & 701,26 & $30 \%$ \\
\hline T7110008 & 2492,51 & $58 \%$ & 783,33 & $26 \%$ \\
\hline T7110009 & 4264,73 & $72 \%$ & 2130,49 & $53 \%$ \\
\hline T7110010 & 3239,52 & $74 \%$ & 1451,13 & $40 \%$ \\
\hline T7110011 & 3845,71 & $71 \%$ & 737,86 & $20 \%$ \\
\hline T7110012 & 3581,57 & $58 \%$ & 157,73 & $30 \%$ \\
\hline T7110013 & 835,24 & $48 \%$ & 1588,86 & $40 \%$ \\
\hline
\end{tabular}

This solution can be appropriated in some cases. However, we observed that the simple combination of the experts does not provide learning and adaptation capacities, which makes it difficult to improve the output of the individual experts. In our proposal, we have implemented two different strategies for the ensemble of information: MLP and SVM. Both are analyzed in detail in the experiments.

We have implemented a hybrid ANN to predict the learning style of the students, which the system received the data from biometrics behavior and used tasks. It is a hybrid prediction method based on MLP and SVM classifier that uses these sources of knowledge and provide the predictions of learning styles for which the system can generate more arguments to justify their suitability (Durães D. , Carneiro, Jimenez, \& Novais, 2018).

It is imperative to mention that these features aim to quantify the student's work and estimate the level of attentiveness. These experiments allow drawing some interesting conclusions about students and their behavior during the assessment. However, this test was implemented in a good class with higher scores in all subjects. Yet, they react in different ways during normal or assessment lessons. We can see that they are more focused on the assessment lesson, so they have a higher attention level in these situations. The teacher needs to improve the results 
the level of attention in normal class because he has an average of $33 \%$ of student work.

This application shows to the teacher that he needs to change the activity; he must improve different approach of the activity or different level of effort in order to increase attentiveness. We also concluded that there are also significant differences in the interaction patterns when comparing the two lessons, as the students react in different ways in a normal and assessment lesson.

The classification tests for the keyboard, mouse and tasks data were carried out splitting the data into training (70\%) and test (30\%) datasets using the Resample filter for each type of lesson detected in each experiment. The training datasets from all the classes are then combined to form the final training dataset. The results obtained are as follows in Table 5.3. It is necessary to remark that the results obtained demonstrate that it is very difficult to predict the class from the data obtained.

Table 5.3 - Classification for keyboard, mouse and tasks.

\begin{tabular}{|c|c|c|c|}
\hline & MLP & SVM & Ensemble \\
\hline Keyboard & $72 \%$ & $81 \%$ & $84 \%$ \\
\hline Mouse & $65 \%$ & $63 \%$ & $69 \%$ \\
\hline Tasks & $83 \%$ & $84 \%$ & $86 \%$ \\
\hline
\end{tabular}

\subsubsection{CASE STUDY 2 - WORK-TASK RELATED}

In this work we compare the same class from a vocational course while performing an activity based on Microsoft Access in a lesson.

\subsubsection{Context}

We have been using it for the past months in the High School of Caldas das Taipas, located in northern Portugal. In this work we compare the same class from a vocational course while performing an activity based on Microsoft Access in a lesson. For this purpose, a group of 14 (all girls) students were selected to participate in this experience. Their average age is 15.9 years old ( $S D=1.5$ years). The experiment was applied in a lesson, where they had access to an individual computer and 100 minutes to complete the task. Students received, at the beginning 
of the lesson, all necessary data with the goals of the task. For this class, the lesson started in the afternoon and students received, at the beginning of the lesson, a document with the goals of the task, which in this case required the use of Microsoft Access and Adobe Acrobat Reader.

\subsubsection{Motivation}

In the context of an organization, the gathering and analysis of metrics describing user behavior, and the providing of tools for visualization (particularly realtime analytics) enables better decision-making and data-driven actions that consider the state and well-being of each individual user. Such initiatives can nowadays be scaled to hundreds or thousands of users, through the use of Big Data tools and techniques, without compromising performance and availability.

In the Portuguese academic context, this system gains increased relevance as current policies move towards the creation of larger classes, which make it increasingly difficult for the teacher to individually address to each student.

\subsubsection{Objectives}

The goal was to determine the level of attention when measured with the work-related task and compared with mouse and keyboard interactions. With these results the smart environment can support future decision-making. We are following several cohorts of students during their academic activities. This data collection

process will allow assessing the influence on attention of aspects such as: breaks, time of day, class contents, and class objectives, among others.

We also show several tools supported by this system that, when at the disposal of the teachers may allow him/her to: decide, in real-time, which students to focus on, according to their level of attention; evaluate, a posteriori, which contents are more prone to generate distraction, providing a chance for improvement; identify, in real-time, fluctuations in attention, improving decision-making concerning aspects such as when to make breaks or when to dismiss the class.

Firstly, it is necessary to know the interaction with the mouse and with the keyboard that each task will have. In some tasks the interaction with the mouse will be higher while in others that will happen with the keyboard, and finally in others, it will practically be the same level of interaction. However, it is difficult to know, a 
priori, what the exact percentage of interaction of the mouse or the keyboard will be. To know these values, we first count the number of times that each key is pressed and the number of times that the mouse is clicked down for each user.

Secondly, we calculated the percentage of the interaction of the mouse, which is the average number of times that the mouse was pressed in the class taking into account the total of interactions with the mouse and the keyboard of the class. Similarly, we calculated the percentage of the interaction of the keyboard, which is the average number of times that the keys were pressed in the class taking into account the total of interactions with the mouse and the keyboard of the class.

\subsubsection{Results}

In Table 5.4 it is presented the average of the interactions of the mouse and keyboard in the class and the global average percentage of the interaction of the mouse $(59 \%)$ and keyboard $(41 \%)$ of the class in this lesson.

Table 5.4 - The average interaction of the mouse and the keyboard of the class for this lesson

\begin{tabular}{cccccc}
\hline & $\begin{array}{c}\text { Key } \\
\text { Pressed }\end{array}$ & $\begin{array}{c}\text { Interaction } \\
\text { Key pressed }\end{array}$ & $\begin{array}{c}\text { Mouse } \\
\text { Clicked }\end{array}$ & $\begin{array}{c}\text { Interaction } \\
\text { Mouse Clicked }\end{array}$ & $\begin{array}{c}\text { Total } \\
\text { Interaction }\end{array}$ \\
\hline $\begin{array}{c}\text { Avera } \\
\text { ge Users }\end{array}$ & 3.875 & $41 \%$ & 5626,64 & $59 \%$ & 9.501 \\
\hline
\end{tabular}

On the order hand, we obtained the amount of time that each student spent at the computer (Task Duration) as well as the amount (and percentage) of time that each student devoted to work and to other activities in the lesson. Table 5.5 allows the teacher to analyze this result.

However, the time that the student spent in the task-related does not indicate the level of attention in some cases, because in some situations the user might have opened the task-related and during that time he did not interact with the computer. In this case, if the level of attention was measured only by the task-related, the student has $100 \%$ of attention and in reality, his attention level should be $0 \%$. For these situations, it is necessary to analyze the amount of interaction with the mouse and the keyboard and cross these data. Based on equation (1), (2), and (3) of chapter 4, we obtain the relative attention presented in Table 5.6. 
Table 5.5 - The amount of time that each student spent interacting with the computer and the amount of actual work versus the amount of time spent interacting with other applications.

\begin{tabular}{rrrr}
\hline Student & Task Duration & Work \% & \multicolumn{1}{c}{ Others $\%$} \\
\hline T2240001 & $85.0084 \mathrm{~min}$ & $0,0 \%$ & $100,0 \%$ \\
\hline T2240002 & $76.0154 \mathrm{~min}$ & $78.7 \%$ & $21.3 \%$ \\
\hline T2240003 & $90.0093 \mathrm{~min}$ & $69,7 \%$ & $30,3 \%$ \\
\hline T2240004 & $90.0065 \mathrm{~min}$ & $56,8 \%$ & $43,2 \%$ \\
\hline T2240005 & $90.0103 \mathrm{~min}$ & $57,0 \%$ & $43,0 \%$ \\
\hline T2240006 & $90.0083 \mathrm{~min}$ & $60,6 \%$ & $39,4 \%$ \\
\hline T2240007 & $95.0146 \mathrm{~min}$ & $61,8 \%$ & $38,2 \%$ \\
\hline T2240008 & $70.0049 \mathrm{~min}$ & $83,7 \%$ & $16,3 \%$ \\
\hline T2240009 & $70.0069 \mathrm{~min}$ & $88,6 \%$ & $11,4 \%$ \\
\hline T2240010 & $80.0047 \mathrm{~min}$ & $61,8 \%$ & $38,2 \%$ \\
\hline T2240011 & $75.0066 \mathrm{~min}$ & $83,7 \%$ & $16,3 \%$ \\
\hline T2240012 & $77.0067 \mathrm{~min}$ & $85.6 \%$ & $14.4 \%$ \\
\hline T2240013 & $70.0048 \mathrm{~min}$ & $75,5 \%$ & $24,5 \%$ \\
\hline T2240014 & $75.006 \mathrm{~min}$ & $70,2 \%$ & $29,8 \%$ \\
\hline
\end{tabular}

It can be observed that the relative attention decreases in general, because the system takes into account the interaction of the user with mouse and keyboard. That is, some-one who spent $100 \%$ of the time using the application that was supposed to but was interacting only $50 \%$ of the time, would have a score of attention of $50 \%$.

Table 5.6 - Relative attention of each user in the lesson.

\begin{tabular}{ccccc}
\hline User & $\begin{array}{c}\text { Relative } \\
\text { MouseDown }\end{array}$ & $\begin{array}{c}\text { Relative } \\
\text { KeyDown }\end{array}$ & Work-related & Relative Attention \\
\hline T2240001 & $31,45 \%$ & $30,22 \%$ & $0,00 \%$ & $0,00 \%$ \\
\hline T2240002 & $31,70 \%$ & $15,59 \%$ & $78,70 \%$ & $37,22 \%$ \\
\hline T2240003 & $16,95 \%$ & $11,92 \%$ & $69,73 \%$ & $20,13 \%$ \\
\hline T2240004 & $48,43 \%$ & $26,55 \%$ & $56,77 \%$ & $42,56 \%$ \\
\hline T2240005 & $21,92 \%$ & $10,92 \%$ & $56,95 \%$ & $18,70 \%$ \\
\hline T2240006 & $43,71 \%$ & $21,75 \%$ & $60,60 \%$ & $39,67 \%$ \\
\hline T2240007 & $17,06 \%$ & $7,11 \%$ & $61,81 \%$ & $14,94 \%$ \\
\hline T2240008 & $44,89 \%$ & $25,05 \%$ & $83,73 \%$ & $58,56 \%$ \\
\hline T2240009 & $25,64 \%$ & $24,13 \%$ & $88,58 \%$ & $44,09 \%$ \\
\hline T2240010 & $24,51 \%$ & $15,61 \%$ & $61,77 \%$ & $24,78 \%$ \\
\hline T2240011 & $38,29 \%$ & $25,50 \%$ & $83,72 \%$ & $53,41 \%$ \\
\hline T2240012 & $32,93 \%$ & $23,36 \%$ & $85,60 \%$ & $48,19 \%$ \\
\hline T2240013 & $45,25 \%$ & $51,57 \%$ & $75,52 \%$ & $73,11 \%$ \\
\hline T2240014 & $33,20 \%$ & $24,65 \%$ & $70,21 \%$ & $40,62 \%$ \\
\hline
\end{tabular}




\subsection{LEARNING STYLE}

After solving the problem of supervising attention, it was studied the Learning styles. The theory of learning styles that will be followed is the Felder-Silverman theory, presented in subsection 3.5.4.

\subsubsection{CASE STUDY 3 - LEARNING STYLE INFLUENCE IN USER BEHAVIOR}

This case study was applied in four different lessons from the same class. The idea is applying different learning style in each lessons and observed the user's behavior.

\subsubsection{Context}

The study took place at the High School of Caldas das Taipas, located in the north of Portugal where assessment activities take place on the computer. In this kind of assessment activities, when students enter the room, they have their computer. Each computer has a keyboard, a mouse, and a screen. For this purpose, a group of 14 (all girls) students was selected to participate in this experience. Their average age is 15.9 years old ( $S D=1.5$ years). Information regarding each assessment's duration is presented in Table 5.7.

Table 5.7 - Summary of the characteristics of each assess activity.

\begin{tabular}{lllll}
\hline & & \multicolumn{3}{c}{ Duration (minutes) } \\
\cline { 3 - 5 } Class & Date & $\bar{x}$ & $\tilde{x}$ & $\mathrm{~S}$ \\
\hline Video & $21-05-2018$ & $7.31612 \mathrm{E}+15$ & $8.4653 \mathrm{E}+15$ & $3.00389 \mathrm{E}+15$ \\
Image & $24-05-2018$ & $9.99167 \mathrm{E}+15$ & $1.21798 \mathrm{E}+16$ & $4.7135 \mathrm{E}+15$ \\
Text & $25-05-2018$ & $6.19021 \mathrm{E}+15$ & $7.29301 \mathrm{E}+15$ & $2.64201 \mathrm{E}+15$ \\
Audio & $29-05-2018$ & $7.0459 \mathrm{E}+15$ & $8.95808 \mathrm{E}+15$ & $3.55391 \mathrm{E}+15$ \\
\hline \hline
\end{tabular}

The assessment activity begins at the same time for all students and they log in the pre-defined software using their personal credentials and the activity begins. The experiment took place in four different multimedia lessons, with a maximum of 100 minutes to complete the assessment. In each assessment, different activity styles for the same subject were applied, where the first lesson was based on exercises related to video editing; the second lesson, the proposed task was related to image editing; in the third lesson, the proposed task was related to text editing; and in the fourth lesson, the proposed task was related to audio editing. 


\subsubsection{Motivation}

In the case of the selected population (vocational students), passing has significant benefits, while failing has important disadvantages. This specific population (vocational students) was selected to participate in this study, given these characteristics, since they are students who easily give up activities and, consequently, the learning and evaluation processes can give up easily.

\subsubsection{Objectives}

Asides from the mentioned behavioral features, which describe the interaction of the student with the computer, the system also registers the application usage by recording the timestamp in which each student switched to a specific application, by recording the user's ID, timestamp and application name. By default, applications that are not considered work-related is count negatively towards the quantification of attention.

The following attention performance features are monitored:

- Activity Timer - time between the start and the completion of the task;

- Main App. Total Time Usage - total time spent in the task solver application (i.e. the Adobe Photoshop app.);

- Main Application Percentage Usage - usage percentage of the task solver

Application.

\subsubsection{Results}

To analyze the students' behavior throughout the assessment lesson activity and, more precisely, the evolution of student's attention and performance, we have obtained these results. After applying a survey to the students, they indicated that their preferred style was: for 70 percent image and for 30 percent was video. Based on this result and in the results obtained in the four assessment lessons, presented in Table 5.8 and Figure 5.3. We can conclude that: (1) the time spent in the application defined by the teacher has the higher level for lesson assessment in the video, which is the preferred style only for 30 percent of the students. In the preferred style of most of the students, image, they have a level of attention of 91,36 , 
lowest average. (2) In case of mouse velocity, the higher level was for the assessment level of video and image.

Based on the preparation process mentioned, we now focus on the relevance of the features analyzed for the prediction of the student's learning method preference. With this work step, it is intended: on the one hand, to identify and remove unnecessary, irrelevant and redundant features of the dataset that do not contribute to the prediction of the student's learning method preference; on the other hand, to verify, within the relevant features, those that present greater relevance, making it possible to order them by degree of importance.

Table 5.8 - Summary of the biometric features variation for each exam.

\begin{tabular}{llllllllll}
\hline Class & CD & DBC & DDC & \multicolumn{2}{l}{ DPLBC KDT } & MA & MV & APP\% & TBK \\
Units & $\mathrm{ms}$ & $\mathrm{px}$ & $\mathrm{px}$ & $\mathrm{ms}$ & $\mathrm{px}$ & $\mathrm{px} / \mathrm{s}^{2}$ & $\mathrm{px} / \mathrm{ms}$ & $\%$ & $\mathrm{~ms}$ \\
\hline Video & 188.31 & 206.1 & 214.24 & 2819.12 & 1002.8 & 0.6811 & 0.5931 & 94.14 & 1865 \\
Image & 150.73 & 150.73 & 161.88 & 3725.35 & 2669.1 & 0.6659 & 0.5413 & 91.36 & 1738 \\
Text & 146.43 & 227.4 & 152.52 & 2838.14 & 1223.0 & 0.6056 & 0.5312 & 89.23 & 1073.1 \\
Audio & 281.0 & 189.2 & 119.10 & 2408.52 & 714.1 & 0.5828 & 0.4986 & 93.7 & 1758 \\
$\bar{x}$ & 187.66 & 227.0 & 165.70 & 2946.41 & 1384.2 & 0.6367 & 0.5447 & 92.08 & 1599.3 \\
\hline \hline
\end{tabular}

In addition, fewer features are desirable for the future development of less complex classification models, allowing for an easier interpretation.

For this study, the filter methods were disregarded, since the lack of direct correlation between a feature and the class (i.e. the student's learning preference) is not enough evidence that the respective feature is not important in conjunction with other features. As such, for the selection of most relevant features, the Boruta technique (a wrapper method built around the random forest classification algorithm) was applied along with the 10 -fold cross-validation process.

The Boruta follows a completely relevant feature selection method, which captures all the attributes that, in some circumstances, are relevant to the outcome variable. In contrast, most traditional algorithms of feature selection rely on a small subset of features that produces a minimum error in a chosen classifier. Its results are shown in Figure 5.4, where the Exam Duration feature is presented as the most relevant for predicting the student's learning preference, followed by the Assessment Grade, the Key Down Time, the Mouse Acceleration, and the Duration Distance Clicks. 

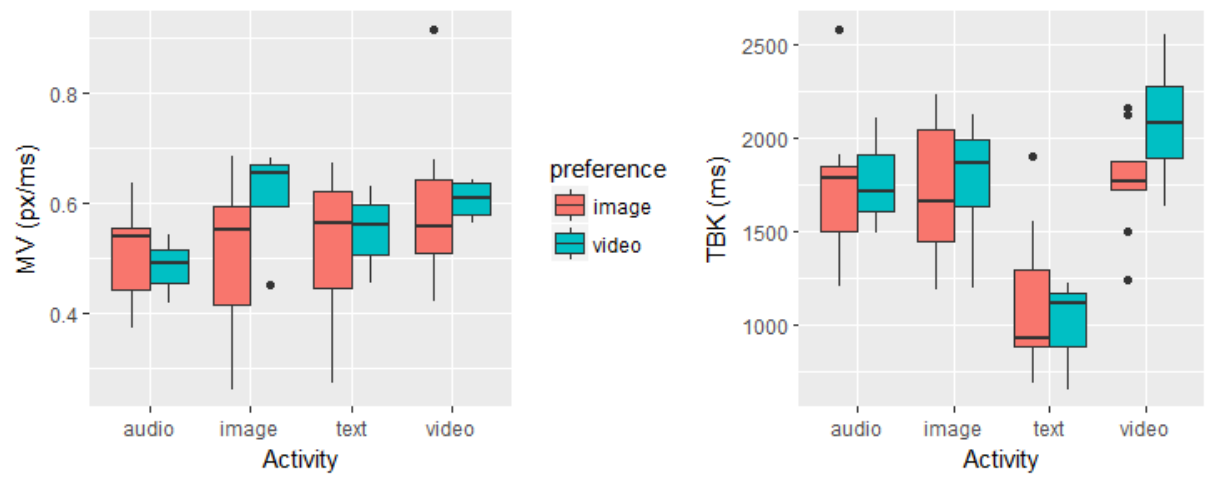

preference

官 image

官 image

审 video

video
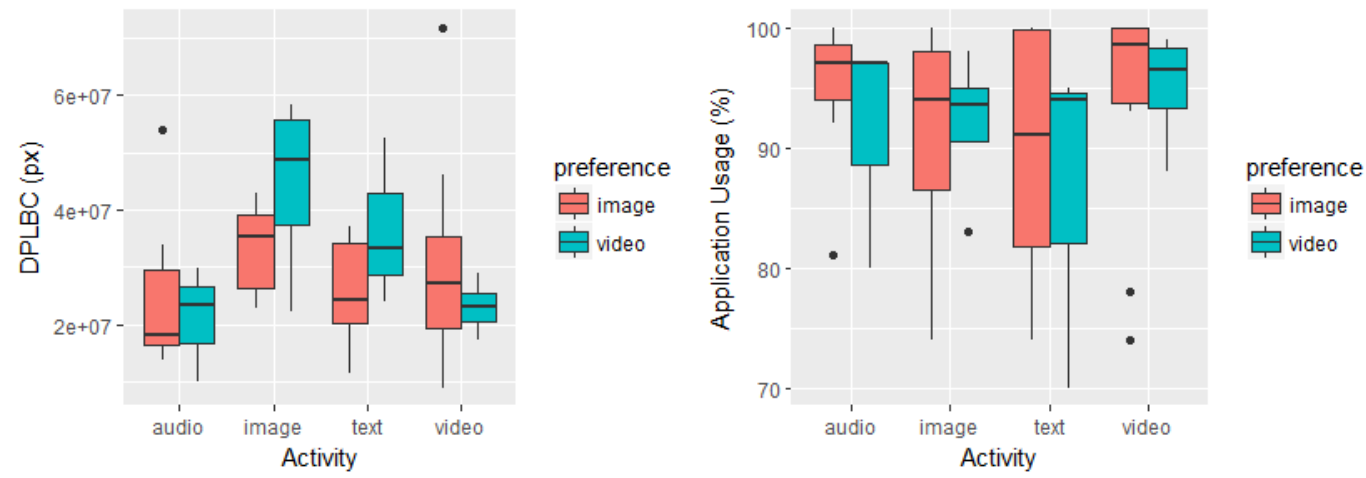

Figure 5.3 - Activity for the four lessons.

Variable Importance

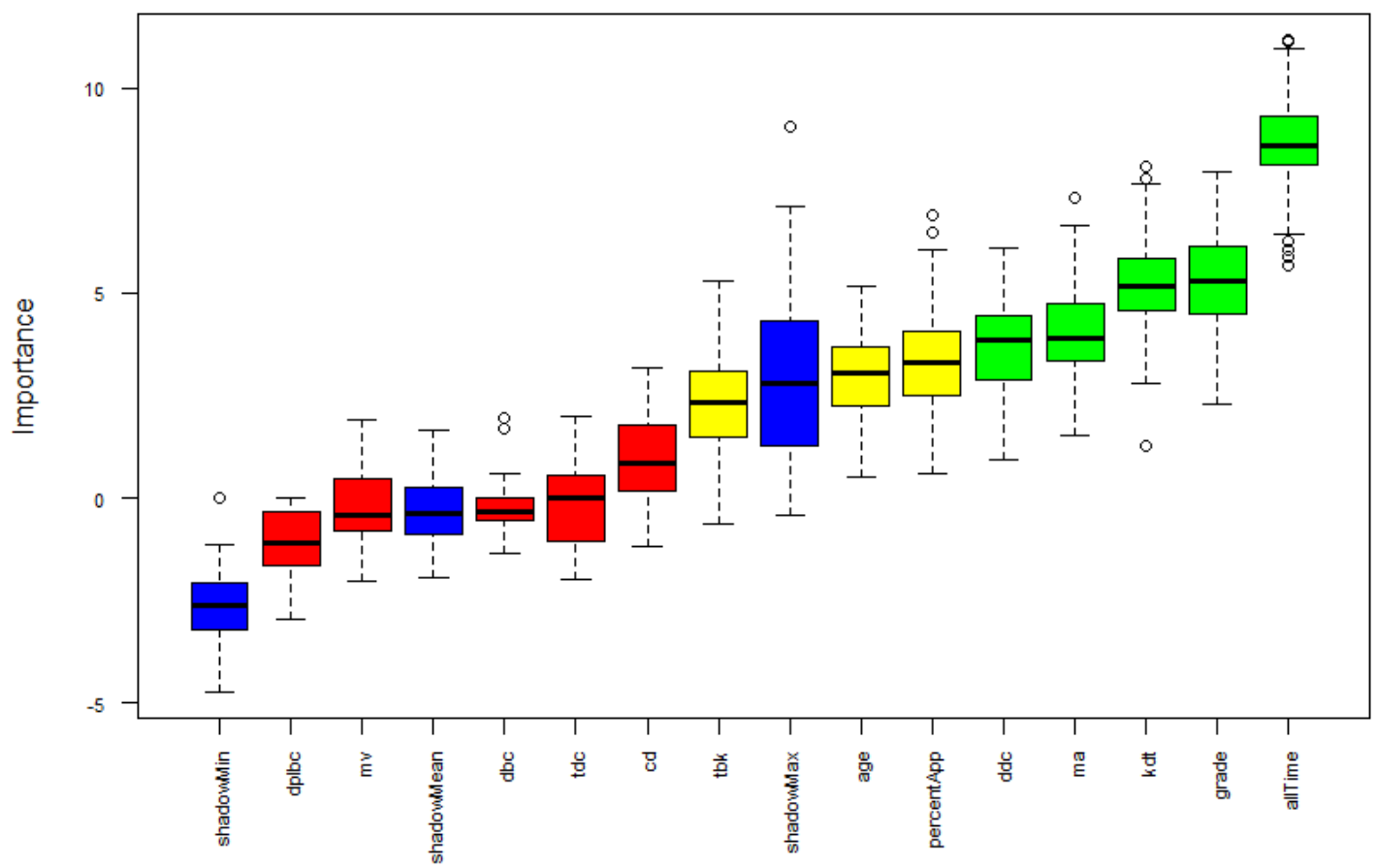

Figure 5.4. - Feature Selection - Boruta Method. 


\subsection{EFFECTS OF USER EMOTIONS}

The idea is understand the underlying mechanisms that lead to performance anxiety and provide their students with the best coping tools and strategies.

\subsubsection{CASE STUDY 4-EFFECTS OF USER EMOTIONS}

We intended to obtain an approach to assess the effects of stress on students during high-stakes exams, which constitute particular stressful moments in the academic career. Specifically, we look at behavioral biometrics and mouse dynamics to develop a non-intrusive method to assess the performance of students while interacting with the computer.

\subsubsection{Context}

The study took place in the School of Medicine of the University of Minho, where exams take place at the computer. In this kind of exams, when students enter the room, they are indicated their computer. Each computer has a keyboard, a mouse and a screen. At the designated time they log in the exam platform using their personal credentials and the exam begins. When the exam ends, students are allowed to leave the room.

Data was collected in four different exams, which took place in different dates, comprising a total of 363 students. Of these, data from 66 students that left the exam in the initial 15 minutes, who were not actually trying to complete the exam, were discarded. The dataset thus includes data from 297 participants out of the initial 363 students $(81.82 \%)$. Table 5.9 presents the summary of the characteristics of each exam.

Table 5.9 - Summary of the characteristics of each exam.

\begin{tabular}{|c|c|c|c|c|c|c|}
\hline \multirow[b]{2}{*}{ Exam } & \multirow[b]{2}{*}{ Date } & \multicolumn{3}{|c|}{ Duration (min) } & \multirow[b]{2}{*}{ \#students } & \multirow[b]{2}{*}{ \#participants } \\
\hline & & $\bar{x}$ & $\tilde{x}$ & $S$ & & \\
\hline $\mathrm{A}$ & $16 / 10 / 2015$ & 74.59 & 47.54 & 53.28 & 104 & 62 \\
\hline B & $18 / 02 / 2016$ & 102.36 & 107.87 & 48.48 & 107 & 90 \\
\hline $\mathrm{C}$ & $19 / 02 / 2016$ & 90.80 & 83.14 & 49.00 & 17 & 17 \\
\hline $\mathrm{D}$ & $28 / 10 / 2015$ & 107.12 & 120.75 & 28.83 & 135 & 128 \\
\hline
\end{tabular}




\subsubsection{Motivation}

The educational organization need to providing the student community with viable solutions for coping with stress. However, it is still necessary to determine which students need this support as they do not always come forward on their own initiative. The identification of these students may not always be easy as people often tend to disguise the consequences of stress. Moreover the professor, who might be in the best position to do so, is often dealing with hundreds of students, making this a rather difficult task to achieve.

\subsubsection{Objectives}

The goal is to analyze how performance varies during high-stakes exams for each individual student, eventually distinguishing between students who are able to maintain a steady performance and students whose performance varies significantly during the duration of the exam.

\subsubsection{Results}

Two major behavioral patterns can be identified, based on ten features that quantify the performance of the student's interaction with the computer: (1) students who are able to sustain performance during the exam and (2) students whose performance varies significantly.

Figure 5.5 depicts the type of information that these features provide. It shows the evolution of the performance of a specific student during an exam through two features: Click Duration and Mouse Velocity. The velocity of the mouse increases until approximately the same point in time and then it starts decreasing. The duration of each click decreases until roughly the middle of the exam and then increases up to a global maximum. Both features point out an initial improvement of performance, followed by a degrading. Figure 5.5 reveals a classical effect of stress: performance tends to improve for some time after the beginning of the stressor stimulus, with a drop off in performance after some time performing above average.

A random decision forest and a neural network to predict exam completion time were trained and evaluated. The encouraging results show, on the one hand, the significance and consistency of the behaviors observed. Indeed, this may constitute a valuable tool for a professor to assess the state of the students while 
taking an exam, eventually intervening to calm them if necessary and possible. If intervening is not possible, which is common in high-stakes exams, the professor still has a valuable tool to assess the magnitude of the effects of this stressful experience on the students.
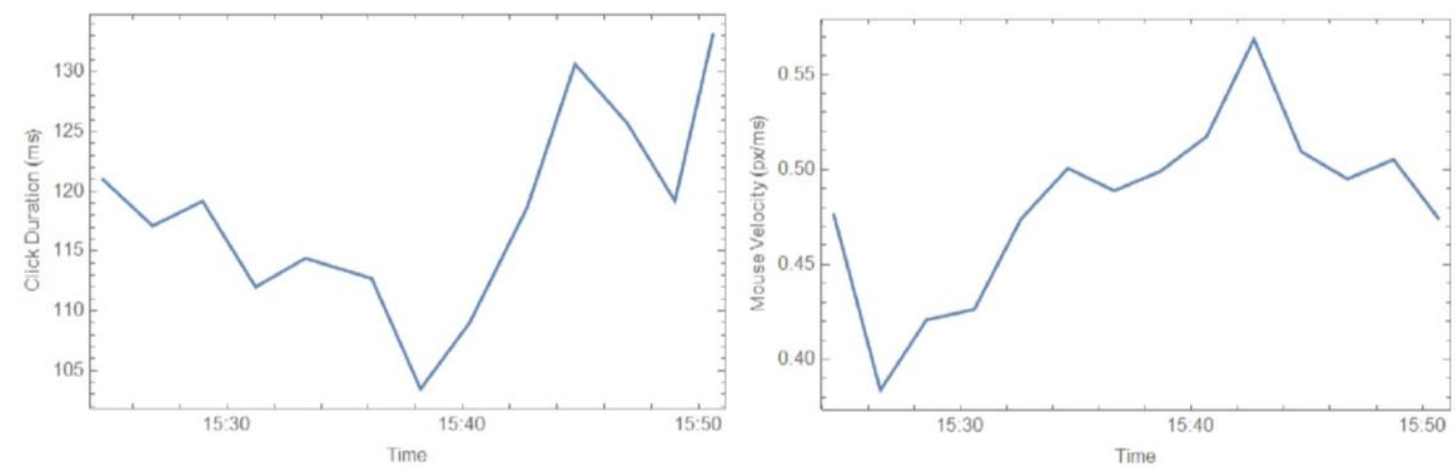

Figure 5.5 - Real-time performance: evolution of one student's interaction performance during the exam. Left: Click Duration. Right: Mouse Velocity.

\subsection{Predict User Behavior}

This case study highlights the main biometric behavioural variations for each activity and bases the set of attributes relevant to the development of machine learning classifiers for the prediction of students' learning preference.

\subsubsection{CASE STUdY 5- ForeCASTING STUdENT'S PREFERENCE IN E- LEARNING SYSTEMS}

We propose an approach of an intelligent e-learning system able to monitor the patterns of students' behavior during e-assessments, to support the teaching procedure within school environments.

\subsubsection{Context}

In the present study, we propose an intelligent learning system able to monitor the patterns of students' behavior during e-assessments, to support the teaching procedure within school environments. Behavior patterns based on mouse dynamics, keystroke, student's attention and e-assessment activity. 
This paper highlights the main biometric behavioral variations for each activity and bases the set of attributes relevant to the development of machine learning classifiers for the prediction of students' learning preference.

\subsubsection{Motivation}

Especially behavior patterns are calculated based on mouse dynamics, keystrokes, student attention, and electronic assessment activity. This approach used a non-intrusive method to assess the preferences of students while interacting with the computer. The hypothesis is that each student may be affected differently by each type of assess activity style and that this approach is a valid one for measuring such differences.

\subsubsection{Objectives}

In this study, the two methods used were max-min normalisation and Z-score normalisation. Min-max normalisation technique is a normalisation strategy which linearly scales a feature value to the range $[0,1]$, based on the minimum and maximum values of the set of observed values. In other words, the minimum value of the feature value is mapped to 0 while the maximum value is mapped to 1 . As for Zscore, this technique is a stand-in for the actual measurement, and they represent the distance of a value from the mean measured in standard deviations. This distribution technique is useful when relating different measurement distributions to each acting as a 'common denominator'. With this, several machine learning categorisation methods were used to predict the student's activity, through the analysis of his/her behavior in HMI.

\subsubsection{Results}

Several classifiers were trained and tested in order to determine the most efficient method to categorise the student's activity. The set of classification methods trained and tested were: Support Vector Machine, Nearest Neighbour, Naive Bayes, Neural Network and Random Forest. As for the validation method, a split validation method was used to determine the classification performance, where $2 / 3$ of the study cases were used for training the classifiers while the remaining $1 / 3$ was used to test it. Table 5.10 presents the set of results for the classifiers performance. 
Looking upon the outcome, some conclusion can be taken into account: 1) according to the trained and tested classification methods, the Random Forest method presents overall the best performance, with a correct percentage of classifications of $87.5 \%$, while Support Vector Machine presents the worst performance; 2) through the application of Feature Scaling techniques, the performance of the applied classifiers showed an improvement between [6.25\%$25 \%$ ], where the greatest improvement is verified in the neural network classifier; 3 ) the performance of the classifiers is dependent on the quality of the features and the total number of case studies analysed (i.e. 48 case studies).

Table 5.10 - Comparative Analysis of Machine Learning Categorisation Performance.

\begin{tabular}{c|ccc} 
& Raw & Min-Max Normalisation Z-score \\
\hline Support Vector Machine & $37.5 \%$ & $43.75 \%$ & $37.5 \%$ \\
Nearest Neighbour & $43.75 \%$ & $62.5 \%$ & $43.75 \%$ \\
Naive Bayes & $68.75 \%$ & $75 \%$ & $68.75 \%$ \\
Neural Network & $31.25 \%$ & $56.25 \%$ & $56.25 \%$ \\
Random Forest & $81.25 \%$ & $87.5 \%$ & $87.5 \%$ \\
\hline
\end{tabular}

Given the set of conclusions, the Random Forest method was selected to categorise student's activity. Additionally, this model was optimised through the application of hyper-parameter optimisation. In other words, in order to optimise the Random Forest's classification performance, it was required to find the optimal number of leafs/features (i.e. between [1-11] leaf's/features) and number of trees (in this study it was modelled between [1-500] trees) that best suit the model and minimises the validation error function.

For this, an exhaustive grid search was used, where it is trained and validated a set of parameter values for each model. In the end, the model with the lowest error rate was the selected one. Figure 5.6 and Figure 5.7 presents the set of results from this process, where it shows that the model displays an average minimised error when the number of decision trees is 80 .

As for the number of leafs/features for each decision tree, 9 was the number that presented the best performance. Moreover, the features relevance of the model is presented, where the Activity Timer (alltime) is by far the most important one to predict the student's activity, followed by the Duration Distance Clicks (ddc), Time 
Between Keys (tbk), Key Down Time (kdt), Distance Point to Line Between Click (dplbc) and Distance Between Click (dbc).

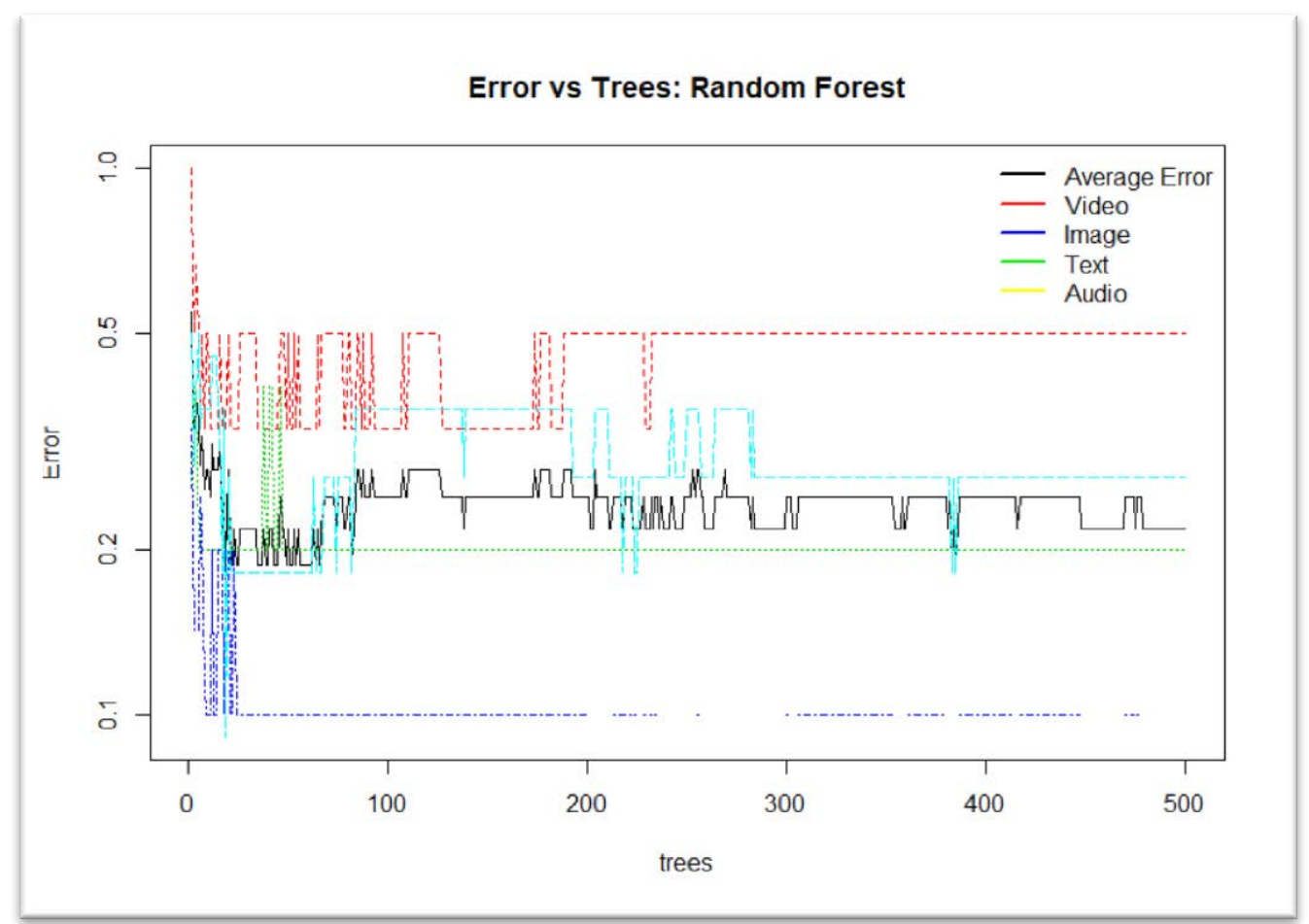

Figure 5.6 - Random Forest: Error vs Trees based on the different labels/activities

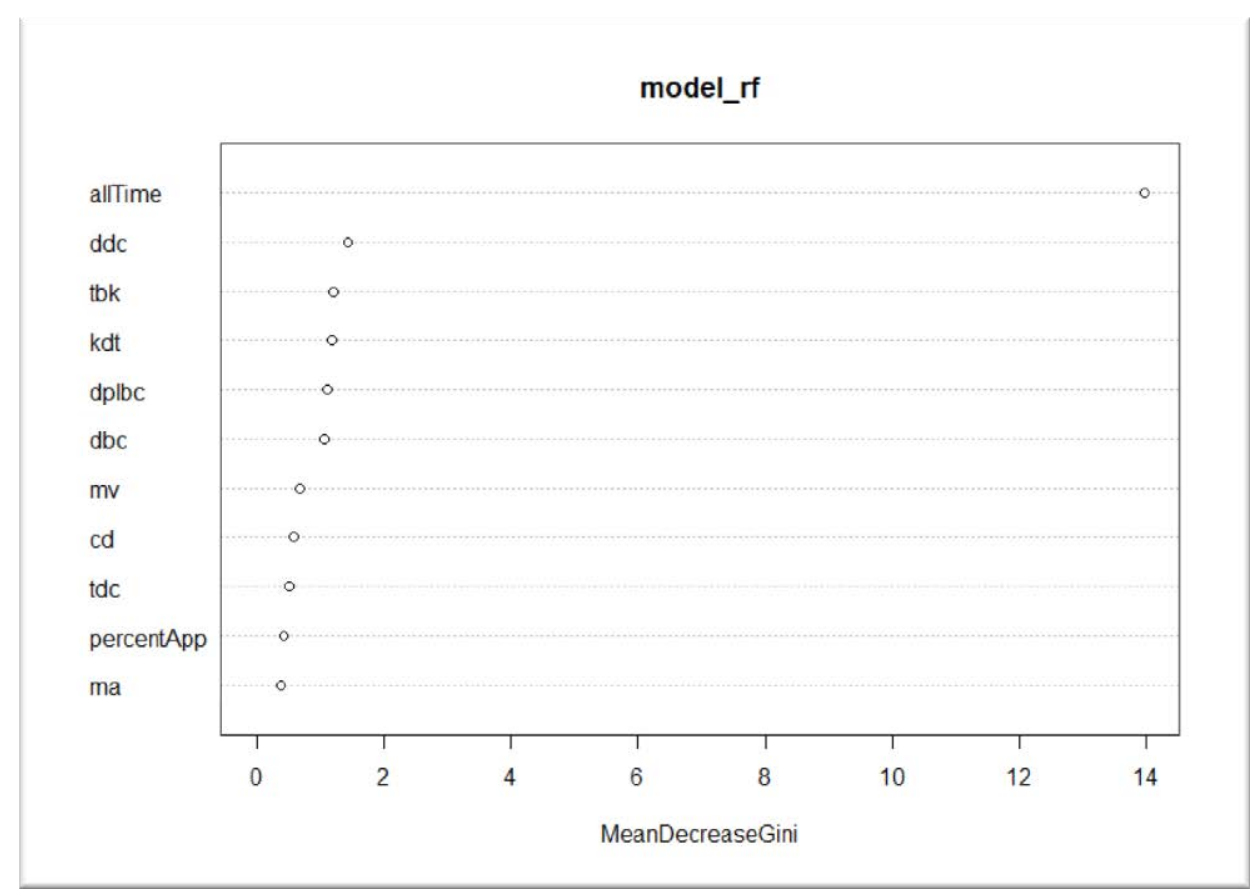

Figure 5.7 - Random Forest: Features Relevance in Activity Classification 
The respective confusion matrix of the Random Forest's model is presented in Table 5.11 where only the Video activity presents a misclassification of $40 \%$ of total cases (i.e. 2/5 cases were misclassified as Audio editing activity).

Table 5.11 - Random Forest: Confusion Matrix.

\begin{tabular}{|c|c|c|c|c|c|}
\hline & Audio & Image & Text & Video & Correct Prediction (\%) \\
\hline Audio & 2 & 0 & 0 & 0 & $100 \%$ \\
\hline Image & 0 & 6 & 0 & 0 & $100 \%$ \\
\hline Text & 0 & 0 & 3 & 0 & $100 \%$ \\
\hline Video & 2 & 0 & 0 & 3 & $60 \%$ \\
\hline
\end{tabular}

\subsection{SUMMARY}

This chapter specifies the evolution of the work during this three years. The case studies are presented by the order they were applied. Firstly began to analyse the attention level. For this subject, two case studies were implemented. Secondly, it was applied a case study about learning style in order to validate the concepts of learning styles. Thirdly, it was implemented a case study about emotions and its effects. The choose emotion was the stress. Finally, it was implemented a case study for predict user behavior where some methods were tested. 
"All truths are easy to understand once they are discovered; the point is to discover them." Galileo Galilei

\section{PUBLICATIONS COMPOSING THE DOCTORAL}

\section{THESIS}

This work deals with the issue of attention monitoring as a form of engagement. With the aim to provide a non-intrusive, reliable and easy tool that can be used freely in the schools or in the organizations, without changing or interfering with the established working routines. We propose an intelligent learning system able to monitor the patterns of students' behavior during lessons, to support the teaching procedure within school environments. The system used behavior patterns based on mouse dynamics, keystroke dynamics, student's attention, and lesson's task activity. The idea highlights the main biometric behavioral variations for each activity and bases the set of attributes relevant to the development of machine learning classifiers for the prediction of student's learning preference. After improving learning systems in a learning and e-learning environment it is possible to predict student's behavior in an (e)learning lesson, based on their interaction with technological devices.

This chapter provides an analysis of some contributions realized during this doctoral thesis in light with the best-published paper. The idea is to present the evolution of the project work published in some important International Conferences and in International Journals. So it is present the summary of some important papers published. 


\subsection{Using Computer Peripheral Devices to Measure ATTENTIVENESS}

Table 6.1 - Conference paper PAAMS 2016

$\begin{array}{ll}\text { Title } & \begin{array}{l}\text { Using Computer Peripheral Devices to Measure } \\ \text { Attentiveness } \\ \text { Dalila Durães, Davide Carneiro, Javier Bajo, Paulo Novais }\end{array} \\ \begin{array}{l}\text { Authors } \\ \text { Publication Type }\end{array} & \begin{array}{l}\text { Conference Proceedings } \\ \text { Trends in Practical Applications of Scalable Multi-Agent } \\ \text { Chapter }\end{array} \\ \begin{array}{l}\text { Systems, the PAAMS Collection } \\ \text { Book series }\end{array} & \begin{array}{l}\text { Advances in Intelligent Systems and Computing } \\ \text { Conference }\end{array} \\ \text { Agth International Conference on Practical Applications of } \\ \text { Publisher } & \text { Springer International Publishing Switzerland } \\ \text { Volume } & 473 \\ \text { Number } & 8 \\ \text { Pages } & \text { 147-155 } \\ \text { Year } & 2016 \\ \text { Month } & \text { June } \\ \text { DOI } & \text { https://doi.org/10.1007/978-3-319-40159-1 12 } \\ \text { Online ISBN } & 978-3-319-40159-1 \\ \text { Print ISBN } & 978-3-319-40158-4 \\ \text { Sate } & \text { Published } \\ \text { SCImago Journal } & \text { IF 0.12 (Q4 - COMPUTER SCIENCE (MISCELLANEOUS)) } \\ \text { Rank (2017) } & \\ \text { Indexation } & \text { ISI Web of Science, Scopus, DBLP }\end{array}$

This paper introduces the attention concept and some features that affect attention. It is also presented a monitoring system using only the peripheral devices of the computer to monitor the user interaction. In this case, a class was monitored in two situations: in a normal lesson and in an assessment lesson. Asides from capturing the interaction of the students with the computer, the monitoring system also registers the applications which students are interacting. We analysed all the applications used by all students and labelled each one of them as belonging to the task or not. We then quantified the amount of time that each student spends interacting with applications related to the task versus other applications. The results thus point out, not only, that it is possible to quantify student attention in a nonintrusive way. We can see that they are more focused on the assessment lesson, so they have a higher level of attention in these situations. We also concluded that there are also significant differences in the interaction patterns when comparing the two lessons. 


\subsection{SUPERVISING AND IMPROVING ATTENTIVENESS IN HUMAN COMPUTER INTERACTION}

Table 6.2 - Conference Paper IE 2016

\begin{tabular}{|c|c|}
\hline Title & $\begin{array}{l}\text { Supervising and Improving Attentiveness in Human } \\
\text { Computer Interaction }\end{array}$ \\
\hline Authors & Dalila Durães, Davide Carneiro, Javier Bajo, Paulo Novais \\
\hline Publication Type & Conference Proceedings \\
\hline Chapter & $\begin{array}{l}\text { Proceedings - 12th International Conference on Intelligent } \\
\text { Environments, IE } 2016\end{array}$ \\
\hline Conference & $\begin{array}{l}\text { 12th International Conference on Intelligent Environments, IE } \\
2016\end{array}$ \\
\hline Publisher & IOS Press BV \\
\hline Number & 10 \\
\hline Pages & $255-264$ \\
\hline Year & 2016 \\
\hline Month & November \\
\hline Online ISBN & $978-1-61499-689-7$ \\
\hline Print ISBN & $978-1-61499-690-3$ \\
\hline Sate & Published \\
\hline $\begin{array}{l}\text { SCImago Journal } \\
\text { rank (2017) }\end{array}$ & 0,15 \\
\hline Indexation & Scopus, DBLP \\
\hline
\end{tabular}

measuring, and supervising the student's level of attentiveness in real scenarios and dynamically provide recommendations to the teacher. This Aml system captures, measures, and supervises the interaction of each student with the computer (or laptop) and indicates the attention level of students in the activities proposed by the teacher. When the teacher has a big class, he/she can visualize in real time the attention level of the students in the proposed activities and acts accordingly.

However, a limitation was also identified that happens whenever a student opens an application that is not task-related and does not interact with the computer anymore until the end of the lesson. In such a case, and according to the collected data, the student's attention during the class quantified is $0 \%$. Similarly, if the student opens a work task-related application and does not interact with the computer after that, the user's attention will be classified as $100 \%$, however, he/she could not be at the computer. In order to address this issue, the system must not only consider the amount of time that each student devoted to the work task-related and to other activities, but also the amount of interaction with the mouse and the keyword. 


\subsection{Characterizing AtTentive Behavior IN INTELLIGENT ENVIRONMENTS}

Table 6.3 Article in journal Neurocomputing 2018

\begin{tabular}{|c|c|}
\hline Title & $\begin{array}{l}\text { Characterizing Attentive Behavior in Intelligent } \\
\text { Environments }\end{array}$ \\
\hline Authors & $\frac{\text { Dalila Durães, Davide Carneiro, Amparo Jiménez, Paulo }}{\text { Novais }}$ \\
\hline Publication Type & Journal \\
\hline Publication Name & Neurocomputing \\
\hline Publisher & Elsevier \\
\hline Volume & 272 \\
\hline Number & 10 \\
\hline Pages & $46-54$ \\
\hline Year & 2018 \\
\hline Month & January \\
\hline DOI & https://doi.org/10.1016/j.neucom.2017.05.091 \\
\hline State & Published \\
\hline $\begin{array}{l}\text { SCImago Journal } \\
\text { Rank (2017) }\end{array}$ & 1.073, Artificial Intelligence Q1 \\
\hline J.C.R. Impact Factor & 3.317 \\
\hline
\end{tabular}
attention level in students. The presented system is able to provide real-time information about each student, as well as information about the class. The system also makes predictions about the best learning style for the student using an ensemble of neural networks. It can be very useful for teachers to identify potentially distracting events and this system might be very useful to the teacher to implement more suited teaching strategies.

We have implemented a hybrid ANN in order to predict the learning style of the students, which the system received the data from biometrics behavior and used task. It is a hybrid prediction method based on MLP and SVM classifier that use these sources of knowledge and provide the predictions of learning styles for which the system is able to generate more arguments to justify their suitability. The output class provide a predicting learning style of each student. When the system has a dataset that are large enough it might performed a more precise classification, classifying the inputs received into different attention levels in real-time. Using this, the teacher could take different measures depending on the profile of the group and/or profile of each student, improving strategies. 


\subsection{MODELLING A SMART ENVIRONMENT FOR NONINTRUSIVE ANALYSIS OF ATTENTION IN THE WORKPLACE}

Table 6.4 - Article in journal Expert System

Title

Authors

Publication Type

Publication Name

Publisher

Volume

Year

Month

DOI

State

SCImago Journal Rank (2017)

J.C.R. Impact Factor
Modelling a smart environment for nonintrusive analysis of attention in the workplace

Dalila Durães, Davide Carneiro, Javier Bajo, Paulo Novais Journal

Expert System

Wiley Online Library

e12275

2018

June

https://doi.org/10.1111/exsy. 12275

Published

0.429, Artificial Intelligence Q2

1.43

The present work adds a new feature to this previously existing framework, by providing for each task the level of interaction of the keyboard and of the mouse. It constitutes a more precise and reliable mechanism for attention monitoring while maintaining, all the advantages of the existing system: non-intrusive, lightweight, and transparent. To obtain the task results, the task rules received in the request are used to get a perception of how much time the user has spent on the applications related to the task rules. As far as the mouse and keyboard results are concerned, their role is present on how the user interaction is occurring and it helps the manager/teacher to understand if any user, who has the application active, is really working on it or not. To do that, we have used the two features one from the keyboard (key downtime) and other from the mouse (time between clicks).

Understanding how attention varies and in which situations the attention varies for each person, it is possible to acts in the right moment and right time, to bring the user's attention level to the ideal value. Using this, the manager may take different measures depending on the profile of the group and/or profile of each user. The classification layer has access to the current and historical state of the group from a global perspective but it can also refer to each user individually. After the classification, the enhanced user's behavior profile is updated in the database behavior profile. 


\subsection{PREDicting COMPLETION TIME IN HIGH-STAKES EXAMS}

Table 6.5 Article in journal Future Generations Computer Systems

$\begin{array}{ll}\text { Title } & \begin{array}{l}\text { Predicting completion time in high-stakes exams } \\ \text { Authors }\end{array} \\ & \begin{array}{l}\text { Davide Carneiro, Paulo Novais, Dalila Durães, José Miguel } \\ \text { Pego, Nuno Sousa }\end{array} \\ \text { Publication Type } & \text { Journal } \\ \text { Publication Name } & \text { Future Generations Computer Systems } \\ \text { Publisher } & \text { Elsevier } \\ \text { Year } & 2018 \\ \text { Month } & \text { February } \\ \text { DOI } & \text { https://doi.org/10.1016/j.future.2018.01.061 } \\ \text { State } & \text { Published } \\ \text { SCImago Journal Rank (2017) } & 0.844, \text { Computer Networks and Communications, Q1 } \\ \text { J.C.R. Impact Factor } & 4.639\end{array}$

In the present study, we analyse student behavior during e-assessment in terms of mouse dynamics. Two major behavioral patterns can be identified, based on ten features that quantify the performance of the student's interaction with the computer: (1) students who are able to sustain performance during the exam and (2) students whose performance varies significantly. Data shows that the behavior of each student during the exam correlates strongly with the time it takes the student to complete it. Several classifiers were trained that predict the completion time of each exam based on the students' interaction patterns. Two of them do it with an average error of around twelve minutes. Results show that there are still mechanisms that can be explored to better understand the complex relationship between stress, performance and human behavior, which can be used for the implementation of better stress detection, monitoring and coping strategies.

Furthermore, a random decision forest and a neural network to predict exam completion time were trained and evaluated. The encouraging results show, on the one hand, the significance and consistency of the behaviors observed. On the other hand, they open the door to a possibility not yet explored, to the extent of our knowledge: the one of predicting, in real-time, the exam completion time. Indeed, this may constitute a valuable tool for a professor to assess the state of the students while taking an exam, eventually intervening to calm them if necessary and possible. 
"When we think differently about the things we are used to seeing daily, we can create innovative opportunities." -George Couros

\section{CONCLUSIONS AND FUTURE WORK}

Throughout the work for this doctoral thesis, the doctoral was aimed at addressing pressing issues in the development of a system that monitors and improves attention. Although it is true that attention was only possible and this factor is a form of engagement, it can be considered that the proposed 3S's framework addresses the limitations identified at the beginning of the document.

This chapter provides an analysis of the contributions of this doctoral thesis in the light of the objectives outlined initially. The content of this chapter explains how the research hypothesis formulated at the beginning of the thesis was validated by the results obtained. There is also a summary of the activities that were carried out to disseminate the results. The chapter concludes with final remarks on the work that has been developed so far and with future work considerations.

\subsection{Contributions}

According to the study hypothesis presented in Section 1.4 and the research questions presented in Section 1.5, it is now possible to present the contribution for each one: 


\section{RQ1: Monitor student's biometric behavior and activity performance}

during lessons.

In chapter 2, section 2.5, concepts and characterization of different types of behavioral biometrics were presented, namely, behavior biometrics non-intrusive and non-invasive. In chapter 4, subsection 4.3.1, 4.3.2, and 4.3.3, concepts and characterization of different types of behavior biometrics were identified. The answer to this request question is explained in chapter 5 , especially in section 5.1 , where the case studies were presented. In this case studies, it was possible to determine each behavioral biometrics of an user and the global behaviour biometric of the class. In this chapter, it was also identified the work-task related of each user in the class and how long the students spend in the work-related task in the class. The first contribution, which is in Section 6.1, was the characterization of attention concept and features that influence attention was defined, based on behaviors biometrics and activity performed.

he main conclusion from this, is that the student's biometric behavior and activity performance during the lessons is possible to obtain based in a non-intrusive and non-invasive method.

RQ2: Characterize student's mouse dynamics, decision-making, attention profile.

A general concept and characterization of behavior biometrics were presented in chapter 2, subsection 2.5.1. Also, a general characterization of attention was presented in chapter 3 , section 3.1 and 3.2. In chapter 4 , section 4.3 , and subsection 4.3.2, concepts and characterization of different types of behavior biometrics were identified. In subsection 4.3.5, a characterization of how to calculate attention level was obtainable. In subsection 4.3.7, was shown the predictions features in order to obtain the decision-making response.

The answer to this request question is explained in chapter 5 , especially:

- For student's mouse dynamics, in subsection 5.1.1, where a case study presented the user behaviour of mouse dynamics. In this case, study it was possible to determine each behavioral biometrics of an user and the 
global behavior biometric of the class in a normal lesson or an assessment lesson.

- For decision-making, in subsection 5.3.1, several classifiers were trained and tested in order to determine the most efficient method to categorise the student' activity. In this subsection is presented how to predict user's behaviour, so that it is possible decision-making.

- For attention profile, in subsection 5.1.2, a case study indicates how to calculate the attention level and to obtain the attention profile of each user and for the class.

The contributions, which are presented in chapter 6 , for:

- Student's mouse dynamics, in section 6.1 and 6.2, where is compared different type of lesson in different classes in order to create a student's mouse dynamics.

- Decision-making, in section 6.5 and 6.6, which it is presented how to predict user's behavior being possible decision-making.

- Attention profile, in section 6.3 and 6.4, indicated how to obtain the attention profile of an user or the global attention profile.

The main conclusion from this is that is possible to characterize student's mouse dynamics, decision-making, and attention profile based in peripheral devices.

RQ3: Test the hypothesis that mouse dynamics and keyboard dynamics can predict a student's attention level.

A general concept and characterization of behaviour biometrics were presented in chapter 2, subsection 2.5.1. Also, a general characterization of attention was present in chapter 3 , section 3.1 and 3.2. In chapter 4, subsection 4.3.7, was shown the predictions features to obtain predictions of attention level.

The answer to this request question is explained in chapter 5 , especially in subsection 5.1.1, where a case study presented the user's behavior of mouse dynamics. In this case study, it was possible to predict the attention level. Also, in section 5.3 was presented a case study of a prediction attention level. 
The contribution, which is presented in chapter 6 , in section 6.3 , where is compared different type of lessons in a different lesson in a class to obtain the prediction attention level.

The main conclusion from this, is that is we can predict a students' attention level based on mouse dynamics and keyboard dynamics.

RQ4: Test the hypothesis that is possible to predict attention level based in different applications of learning style.

In chapter 3, section 3.5 it is showed the different type of learning style and the different theories of learning style. The goal of this feature is to improve the level of attention of the user's based on his/her learning styles. In chapter 4, subsection 4.3.6, it is presented methods to classify learning style. In chapter 5 , section 5.2 , it was presented a case study of the influence of learning style in attention level and how to predict for each student. The contribution is presented in Section 6.3, describing how attention varies from different learning style.

The main conclusion from this, is that is possible to predict attention level based in different applications of learning style, because students have their learning preferences.

RQ5: Create adequate prediction and classification based on emotional affects.

In chapter 2, sections 2.3 and 2.4 it is presented the theoretical foundations of emotions. In chapter 3 , section 3.2 it was exposed some factors that affect attention, namely emotions. In chapter 4 , subsection 4.3 .4 , it is showed how some emotions affect the user interactions of peripheral devices. The contribution is presented in chapter 6, section 6.5, which describes how stress affects the user pattern interaction.

The main conclusion from this, is that is possible to make prediction and classification based on some emotional affects. 
RQ6: Apply the prediction model on (e)learning platforms for noninvasive monitoring.

In chapter 3 , subsection 3.4 it is presented the theoretical foundations of learning. Chapter 4 presented the framework model for learning and e-learning platforms. This framework is a non-invasive and a non-intrusive tool, that monitoring the user's behaviour. In chapter 5, subsection 5.1.2 explains the applied case study, monitoring students. Chapter 6 , section 6.2 and 6.4 are the main contributions presented.

The main conclusion from this is that is possible to have a prediction model on (e)learning platforms with a non-invasive monitoring.

As a complement to this section Table 7.1, shows the objectives for the thesis, as they were defined in Section 1.5 and document sections where they were achieved.

Table 7.1 - Reply to the initial Objectives.

\begin{tabular}{|c|c|}
\hline Objective & Chapter/Section \\
\hline $\begin{array}{l}\text { Objective 1: Monitor students' } \\
\text { biometric behavior and activity } \\
\text { performance during lessons. }\end{array}$ & $\begin{array}{l}\text { Section 2.5, User Behavior, Subsection 4.3.1, Features } \\
\text { Extraction, Subsection 4.3.2, Analytics, Subsection } \\
\text { 4.3.3, User Behavior, Section 5.1, Attention Level, and } \\
\text { section 6.1, Using Computer Peripheral Devices to } \\
\text { Measure Attentiveness. }\end{array}$ \\
\hline $\begin{array}{lcr}\text { Objective } & \text { 2: } & \text { Characterize } \\
\text { student's } & \text { mouse } & \text { dynamics, } \\
\text { decision-making, attention profile. }\end{array}$ & $\begin{array}{l}\text { Subsection 2.5.1, Pattern Interactions using Behavior } \\
\text { Biometrics, Section, 3.1, Attention Concept, Section } \\
\text { 3.2, Features that influence attention, Section 4.3, 3S's } \\
\text { - Seamless Supervising aSsitant, Subsection } 4.3 .2 \text {, } \\
\text { Analytics, Subsection 4.3.5, Classification of Attention } \\
\text { Level, Subsection 4.3.7, Predictions Features, } \\
\text { Subsection 5.1.1, Case Study } 1 \text { - Normal and } \\
\text { Assessment Lesson, Subsection 5.1.2, Case Study } 2- \\
\text { Work-Task Related, and Subsection } 5.3 .1 \text {, Case Study } \\
4 \text { - Predictions. Section } 6.1 \text {, Using Computer } \\
\text { Peripheral Devices to Measure Attentiveness, Section } \\
6.2 \text {, Supervising and Improving Attentiveness in } \\
\text { Human Computer Interactions, Section } 6.3 \text {, }\end{array}$ \\
\hline
\end{tabular}




\begin{tabular}{|c|c|}
\hline & $\begin{array}{l}\text { Characterizing Attentive Behavior in Intelligent } \\
\text { Environment; Section 6.4, Modelling a Smart } \\
\text { Environment for Non-intrusive Analysis of Attention in } \\
\text { the Workplace, and Section } 6.5 \text {, Predictions } \\
\text { Completion Time in High-Stakes Exams. }\end{array}$ \\
\hline $\begin{array}{l}\text { Objective 3: Test the hypothesis } \\
\text { that mouse dynamics and } \\
\text { keyboard dynamics can predict a } \\
\text { students' attention level. }\end{array}$ & $\begin{array}{l}\text { Subsection 2.5.1, Pattern Interactions using Behavior } \\
\text { Biometrics, Section 3.1, Attention Concept, Section } \\
\text { 3.2, Features that Influence Attention, Subsection } \\
\text { 4.3.7, Predictions Features, Subsection 5.1.1, Case } \\
\text { Study } 1-\text { Normal and Assessment Lesson, and } \\
\text { Section 5.3, Predicting Features. Section 6.3, } \\
\text { Characterizing Attentive Behavior in Intelligent } \\
\text { Environment. }\end{array}$ \\
\hline $\begin{array}{l}\text { Objective 4: Test the hypothesis } \\
\text { that is possible to predict attention } \\
\text { level based in different applications } \\
\text { of learning style. }\end{array}$ & $\begin{array}{l}\text { Section 3.5, Learning Styles, Subsection 4.3.6, } \\
\text { Learning Styles's Identification, and Section 5.2, } \\
\text { Learning Style. Section 6.3, Characterizing Attentive } \\
\text { Behavior in Intelligent Environment. }\end{array}$ \\
\hline $\begin{array}{l}\text { Objective 5: Test the hypothesis } \\
\text { that emotion affects user behavior. }\end{array}$ & $\begin{array}{l}\text { Section 2.3, Affective Learning, Section } 2.4 \text {, Affective } \\
\text { Learning, Section } 3.2 \text {, Features that Influence } \\
\text { Attention, and Subsection } 4.3 .4 \text {, Emotions. Section } \\
6.5 \text {, Predictions Completion Time in High-Stakes } \\
\text { Exams. }\end{array}$ \\
\hline $\begin{array}{l}\text { Objective 6: Apply the prediction } \\
\text { model on (e)learning platforms for } \\
\text { non-invasive monitoring. }\end{array}$ & $\begin{array}{l}\text { Section 3.4, Learning, Chapter 4, 3S's Architecture, } \\
\text { and Subsection 5.1.2, Case Study } 2 \text { - Work-Task } \\
\text { Related. Section 6.2, Supervising and Improving } \\
\text { Attentiveness in Human Computer Interactions, and } \\
\text { Section 6.4, Modelling a Smart Environment for Non- } \\
\text { intrusive Analysis of Attention in the Workplace. }\end{array}$ \\
\hline
\end{tabular}

\subsection{VALIDATION OF THE RESEARCH HYPOTHESIS}

To validate the research hypothesis formulated in Section 1.4, it is necessary to demonstrate how the 3S's framework based on a distributed system works. This framework monitored the level of attention, the work task-related in learning activities (his/her engagement), create learning frequent behaviour patterns for each user and can predict user features. 
The approach followed in this project thus relies on the mouse and on the keystrokes dynamics, and on the work-related task to put forward a completely nonintrusive method for assessing attention in $\mathrm{HCl}$. Improving the level of attention is a form to improving engagement.

The 3S's architecture highlights the main biometric behavioural variations for each activity and it is based on a set of attributes relevant to the development of machine learning classifiers for the prediction student's learning preference. Results show that there are still mechanisms that can be explored to understand better the complex relationship between human behaviour, attention, and assessment which could be used for the implementation of better learning strategies. These results might be crucial for improving the learning systems in an (e)learning environment and for predicting student's behaviour activity, based on their interaction with technological devices.

\subsection{DISSEMINATION OF RESULTS}

Since the beginning of this $\mathrm{PhD}$ project and as outlined in the research methodology of Section 1.6, efforts were directed towards achieving a high level of scientific dissemination through journals, conferences, and other media. The organization of scientific meetings was also part of these efforts, along with the supervision of master students and lecturing. These activities were undertaken in order to consolidate knowledge and to exchange ideas. In order to demonstrate the reach of the work developed in this doctoral thesis, the many activities of result dissemination are described in this section.

\subsubsection{Other Publications}

Besides the publications included in this thesis (Chapter 6), it was possible to achieve other publications in international journals and scientific meetings in the fields of Computer Science, Artificial Intelligence, and Education. 


\section{Books with peer review}

$>$ P. Novais, J.J. Jung, G. Villarrubia, A. Fernández-Caballero, E. Navarro, P. González, D. Carneiro, A. Pinto, A.T. Campbell, D. Durães, "Ambient Intelligence - Software and Applications -, $9^{\text {th }}$ International Symposium on Ambient Intelligence", Springer International Publishing.

\section{Chapters in Books with peer review}

> T.S. Martinez, D. A. Durães, F. J. H. Lucena, J. J. R. D. Guardia, "Management Conflicts in E-Learning Environment: Vulnerabilities in ELearning Environments", in Interdisciplinary Perspectives on Contemporary Conflict Resolution, P. Novais, D. Carneiro, (Eds), IGI Global, ISBN: 9781522502456, 2016.

> Durães, D., Bajo, J., Novais, P., "Characterize a Human-Robot Interaction Robot Personal Assistance", in Personal Assistants: Emerging Computational Technologies, Angelo Costa Vicente Julian Paulo Novais (Eds), Springer, pp 135-147, 2017.

\section{Workshop in Books per review - Organizing Committee}

$>$ FESAml: I Future Environments and Solutions for Ambient Intelligence, Intelligent Systems Design and Applications 16th International Conference on Intelligent Systems Design and Applications (ISDA 2016) held in Porto, Portugal, December 16-18, 2016.

$>$ FESAml:Il Future Environments and Solutions for Ambient Intelligence, Ambient Intelligence - Software and Applications - 8th International Symposium on Ambient Intelligence (ISAmI 2017), Advances in Intelligent Systems and Computing, Volume 615, Springer.

> TLAmIS: I Workshop on Teaching and Learning with Ambient Intelligence Systems, Ambient Intelligence - Software and Applications - 9th International Symposium on Ambient Intelligence (ISAml 2018), Advances in Intelligent Systems and Computing, Springer.

\section{International Journal}

Duraes D., Prieta F., Novais P., Enriching Behaviors Patterns with Learning Styles using Peripheral Devices, Knowledge and Information Systems Journal, 
Springer, ISSN: 0219-1377, 2018 (Accepted). ISI JCR (2017) IF 2.247 (Q2 COMPUTER SCIENCE, ARTIFICIAL INTELLIGENCE) SCImago SJR (2017) 0.726 (Q1 - COMPUTER SCIENCE, INFORMATION SYSTEMS)

\section{Conference Proceedings}

Durães, D., Jimenez, A., Bajo, J., Novais, P., Monitoring Level Attention Approach in Learning Activities, in Methodologies and Intelligent Systems for Technology Enhanced Learning, Springer Verlag, Mauro Caporuscio, Fernando De la Prieta, Tania Di Mascio, Rosella Gennari, Javier Gutiérrez Rodríguez, Pierpaolo Vittorini (Eds), Vol. 478, pp 33 - 40, ISBN: 978-3-319-40164-5, 2016, https://doi.org/10.1007/978-3-319-40165-2.

SCImago SJR IF 0.12 (Q4 - COMPUTER SCIENCE (MISCELLANEOUS)) Indexed: ISI Web of Science, Scopus, DBLP.

> Carneiro, D., Durães, D., Bajo, J., Novais, Non-intrusive Monitoring of Attentional Behavior in Teams, in International Symposium on Intelligent and Distributed Computing IDC 2016: Intelligent Distributed Computing X, Springer Verlag, Costin Badica, Amal El Fallah Seghrouchni, Aurélie Beynier, David Camacho, Cédric Herpson, Koen Hindriks, Paulo Novais (Eds), Vol. 678, pp 153 - 162, 2017, ISBN: 978-3-319-48828-8, https://doi.org/10.1007/978-3-319-48829-5.

Scopus (Document Type: Conference Paper), DBLP, ISI Web of Science

> Durães, D., Gonçalves, S., Carneiro, D., Bajo, J., Novais, Detection of Behavioral Patterns for Increasing Attentiveness Level, in International Conference on Intelligent Systems Design and Applications ISDA 2016: Intelligent Systems Design and Applications, Springer Verlag, Ana Maria Madureira, Ajith Abraham, Dorabela Gamboa, Paulo Novais (Eds), Vol. 557, pp 592 - 601, 2017, ISBN: 978-3-319-53479-4, https://doi.org/10.1007/978-3-31953480-0.

Scopus (Document Type: Conference Paper), DBLP, ISI Web of Science

> Durães, D., Cardoso, C., Bajo, J., Novais, P., Learning Frequent Behaviors Patterns in Intelligent Environments for Attentiveness Level, in International Conference on Practical Applications of Agents and Multi-Agent Systems PAAMS 2017: Trends in Cyber-Physical Multi-Agent Systems. The PAAMS Collection - 15th International Conference, PAAMS 2017, Springer Verlag, Fernando De la Prieta, Zita Vale, Luis Antunes, Tiago Pinto, Andrew T. 
Campbell, Vicente Julián, Antonio J.R. Neves, María N. Moreno (Eds), Vol. 619, pp 139-147, 2017, ISBN: 978-3-319-61577-6, https://doi.org/10.1007/978-3-31961578-3.

SCImago SJR IF 0.12 (Q4 - COMPUTER SCIENCE (MISCELLANEOUS)) Indexed: ISI Web of Science, Scopus, DBLP.

$>$ Durães, D., Bajo, J., Novais, P., Analysis Learning Styles Though Attentiveness, in International Conference in Methodologies and intelligent Systems for Techhnology Enhanced Learning MIS4TEL 2017: Methodologies and Intelligent Systems for Technology Enhanced Learning, Springer Verlag, Pierpaolo Vittorini, Rosella Gennari, Tania Di Mascio, Sara Rodríguez, Fernando De la Prieta, Carlos Ramos, Ricardo Azambuja Silveira (Eds), Vol. 617, pp 90-97, 2017, ISBN: 978-3-319-60818-1, https://doi.org/10.1007/978-3-319-60819-8.

SCImago SJR IF 0.12 (Q4 - COMPUTER SCIENCE (MISCELLANEOUS)) Indexed: ISI Web of Science, Scopus, DBLP.

$>$ Durães, D., Castro, D., Bajo, J., Novais, P., Modelling an Intelligent Interaction System for Increasing the Level of Attention, in International Symposium on Ambient Intelligence ISAml 2017: Ambient Intelligence- Software and Applications - 8th International Symposium on Ambient Intelligence (ISAml 2017), Springer Verlag, Juan F. De Paz, Vicente Julián, Gabriel Villarrubia, Goreti Marreiros, Paulo Novais (Eds), Vol. 615, pp 210-217, 2017, ISBN: 978-3319-61117-4, https://doi.org/10.1007/978-3-319-61118-1.

SCImago SJR IF 0.12 (Q4 - COMPUTER SCIENCE (MISCELLANEOUS)) Indexed: ISI Web of Science, Scopus, DBLP.

$>$ Durães, D., Analide, C., Bajo, J., Novais, P., Quantifying the Effects of Learning Styles on Attention, in World Conference on Information Systems and Technologies WorldCIST 2017: Recent Advances in Information Systems and Technologies, Springer Verlag, Álvaro Rocha, Ana Maria Correia, Hojjat Adeli, Luís Paulo Reis, Sandra Costanzo(Eds), Vol. 570, pp 711-721, 2017, ISBN: 9783-319-56537-8, https://doi.org/10.1007/978-3-319-56538-5.

Scopus (Document Type: Conference Paper), DBLP, ISI Web of Science

$>$ Durães, D., Bajo, J., Novais, P., Assess and Enhancing Attention in Learning Activities, in International Conference on Interactive Collaborative Learning ICL 2017: Teaching and Learning in a Digital World, Springer Verlag, Michael E. 
Auer, David Guralnick, Istvan Simonics (Eds), Vol. 715, pp 803-811, 2018, ISBN: 978-3-319-73209-1, https://doi.org/10.1007/978-3-319-73210-7.

Scopus (Document Type: Conference Paper), ISI Web of Science

$>$ Durães, D., Bajo, J., Novais, P., Supervising Attention in an E-learning System, in International Symposium on Ambient Intelligence ISAml 2018: Ambient Intelligence- Software and Applications - 9th International Symposium on Ambient Intelligence (ISAml 2018).

SCImago SJR IF 0.12 (Q4 - COMPUTER SCIENCE (MISCELLANEOUS)) Indexed: ISI Web of Science, Scopus, DBLP.

> Toala, R., Gonçalves, F., Durães, D., Novais, P., Adaptive and Intelligent Mentoring to Increase User Attentiveness in Learning Activities, in 16th IberoAmerican Conference on Artificial Intelligence IBERAMIA 2018 (accepted).

$>$ Novais, P., Durães, D., Gonçalves, F., Forecasting Student's Preference in Elearning Systems, in AI-2018 Thirty-eighth SGAI International Conference on Artificial Intelligence (accepted).

\subsubsection{PARTICIPATION AND ORGANIZATION OF EVENTS}

> MIS4TEL2017 - 4th International Conference in Methodologies and intelligent Systems for Techhnology Enhanced Learning (MIS4TEL'17), 2017.

$>$ Special Session "on Future Environments and Solutions for Ambient Intelligence (FESAml) in "16th International Conference on Intelligent Systems Design and Applications (ISDA'16), General Chair.

$>$ Special Session "on Future Environments and Solutions for Ambient Intelligence (FESAmI) in "ISAMI17 - 8th International Conference on Ambient Intelligence", General Chair.

$>$ I Workshop on Teaching and Learning with Ambient Intelligence Systems (TLAmIS) in "ISAMI18 - 8th International Conference on Ambient Intelligence", General Chair.

\subsubsection{SUPERVISION OF STUDENTS}

During the doctoral programme, the doctoral candidate successfully cosupervised master students and $\mathrm{PhD}$ students at the University of Minho that developed work in the context of this thesis. All these works were supervised by 
Professor Paulo Jorge Freitas de Oliveira Novais. The list of students and their respective dissertation titles are the following:

\section{MSc Co-Supervision}

Completed:

> Catarina Torres Cardoso, Learning Frequent Behaviors Patterns in Intelligent Environments for Attentiveness Level in Computer Sciences Department at University of Minho, Portugal with supervision Paulo Novais, 24/11/2017.

On-going:

$>$ David Barbosa Castro, Modelling and Intelligent Interaction System for Increasing the Level of Attentiveness and Engagement, in Computer Sciences Department at University of Minho, Portugal with supervision Paulo Novais, started 2016.

\subsection{FINAL REMARKS AND FUTURE WORK CONSIDERATIONS}

With this doctoral thesis, it was possible to research some of the most interesting aspects of user's behaviour. The results obtained in the case studies implemented show that this area of action has several possibilities of application. Although in the current work, the focus was directed to the process of learning, it is clear that from this scope it will be possible to apply these tools for decision-making. We possibly will have the need to analyse behavior in the routine tasks of a user in their workplace.

During this work, some obstacles were encountered which were not fully overcome. One of them was at testing in real scenarios. It was possible to apply in one high school, but we were very limited, because on some occasion the Internet does not work or fail, and all the work was lost. Another aspect was the necessary authorization to conduct these tests. The privacy of the students must be guaranteed and the teacher wants to lose his/her free time to prepare the classroom. 
In addition, as future work, we intend to apply this knowledge to the construction of a classification model capable of predicting, in an effective and nonintrusive way, student's learning preferences in real-time.

Conducting this kind of study with a broader population, and in different schools will possibly allow to define more precisely, or even define new types of user's behavior. In the future work, it is also interesting to perform a pilot study involving at least one group of workers.

Although, the door is open to intelligent platforms that allow analysing user's profiles, taking into account their individual characteristics, and to propose new strategies and actions. By providing instructors with access to this information, we allow them to better manage their interactions with the students, namely by pointing out the most problematic cases of inattention in real-time. 
“"'Those who pass by us, do not go alone, and do not leave us alone; they leave a bit of themselves, and take a little of us." - Antoine de Saint-Exupéry

\section{$\mathrm{Bi}$}

\section{BIBLIOGRAPHY}

Gottlieb, J. (2012). Attention, learning, and the value of information. Neurocomputing, 76(2) , 281-295.

Simola, J., Hyönä, J., \& Kuisma, J. (2015). Perception of visual advertising in different media: from attention to distraction, persuasion, preference and memory. Frontiers Media SA.

Albastroiu, R., Anisia, I., Gonçalves, F., Mihaescu, M., \& Novais, P. (2018). An eExam Platform Approach to Enhance University Academic Student's Learning Performance. In J. Del Ser, E. Osaba, M. Bilbao, J. SanchezMedina, M. Vecchio, \& X. Yang, ntelligent Distributed Computing XII (pp. 404-413). Springer, Cham.

Alepis, E., Stathopoulou, I., Virvou, M., Tsihrintzis, G., \& Kabassi, k. (2010). Audiolingual and visual-facial emotion recognition: Towards a bi-modal interaction system. Proceedings International Conference on Tools with Artificial Intelligence, ICTAI, 2, (pp. 274-281). 
Andrade, F., Novais, P., Carneiro, D., Zeleznikow, J., \& Neves, J. (2009). Using BATNAs and WATNAs in Online Dispute Resolution. In K. Nakakoji, Y. Murakami, \& E. McCready, New Frontiers in Artificial Intelligence (pp. 5-18). Tokyo, Japan: Springer, Berlin, Heidelberg.

Anil, K. (2010). Data clustering: 50 years beyond K-means. Pattern Recognition Letters, Vol. 31, Issue 8, 651-666.

Araújo, L. C., Sucupira, L. H., Lizarraga, M. G., Ling, L. L., \& Yabu-Uti, J. B. (2005). User authentication through typing biometrics features. IEEE transactions on signal processing, 53(2):851-855.

ATTENTION-DEFICIT, S., \& et al. (2011). Pediatrics.

Augusto, J., Callaghan, V., Cook, D., \& Kameas, A. (2013). Intelligent environments: a manifesto. Human-Centric Computing and Information Sciences 3(1), 1-18.

Azevedo, R. (2015). Defining and Measuring Engagement and Learning in Science: Conceptual, Theoretical, Methodological, and Analytical Issues. Educational Psychologist 50:1, 84-94.

Bajo, J., Paz, J., Rodríguez, S., \& González, A. (2011). A new clustering algorithm applying a hierarchical method neural network. Logic Journal of the IGPL, 19(2), 304-314.

Banse, R., \& Sherer, K. (1996). Acoustic profiles in vocal emotion expression. Journal of Personality and Social Psychology, 70 (3), 614-636.

Bartlett, F. (1943). Ferrier Lecture: Fatigue Following Highly Skilled Work. Proceediings of the Royal Society of London. Series B-Biological Sciences 131(863), 247-257.

Benevenuto, F., Rodrigues, T., Meeyoung, C., \& Almeida, V. (2009). Characterizing user behavior in online social networks. Proceeding IMC '09 Proceedings of the 9th ACM SIGCOMM conference on Internet measurement (pp. 49-62). New York: ACM. 
Best, R., Floyd, R., \& McNamara, D. (2008). Taxonomy of educational objectives. In E. D. McKay, Handbook 1:Cognitive domain. Reading Psychology (Vol. 29). Addison Wesley Publishing.

Bishop, S. (2008). Trait Anxiety and Impoverished Prefrontal Control of Attention. Nature Neuroscience, 12, 92-98.

Black, C., O'Connor, P., \& McCully, K. (2005). Increased daily physical activity and fatigue symptoms in chronic fatigue syndrome. Dynamic Medicine 4(1), 1.

BORRAJO, M., Baruque, B., Corchado, E., Bajo, J., \& Corchado, J. (2011). Hybrid neural intelligent system to predict business failure in small-to-medium-size enterprises. International journal of neural Systems, 21(04) 277-296.

Bradley, M., \& Lang, P. (1999). Affective Norms for English Words ( ANEW ): Instruction Manual and Affective Ratings. University of Florida: Psychology (Vol. Technical). The Center for Research in Psychophysiology.

Bull, P. (1987). Posture and Gesture. Pergamon Press.

Bybee, R. (1989). Science and technology education for the elementary years: Frameworks for curriculum and instruction. Office of Educational Research and Improvement (ED), p. 155.

Calvo, R., Member, S., \& Mello, S. (2010). Affect Detection: An Interdisciplinary Review of Models , Methods, and their Application to Learning Environments. Review Literature And Arts Of The Americas, 1(1), 1-23.

Carneiro, D., \& Novais, P. (2017). Quantifying the effects of external factors on individual performance. Future Generation Comp. Syst., 66, 171-186.

Carneiro, D., Durães, D., Bajo, J., \& Novais, P. (2016). Non-intrusive Monitoring of Attentional Behavior in Teams. In C. Badica, \& et al., International Symposium on Intelligent and Distributed Computing (IDC 2016): Intelligent Distributed Computing $X$ (pp. 153-162). Switzerland: Springer Cham.

Carneiro, D., Novais, P., Pêgo, J., Sousa, N., \& Neves, J. (2015). Using Mouse Dynamics to Assess During Online Exams . Hybrid Artificial Intelligent Systems, vol. 9121, 345-356. 
Carneiro, D., Pimenta, A., Gonçalves, S., Neves, J., \& Novais, P. (2015). Monitoring and improving performance in human-computer interaction. Wiley InterScience, 1-20.

Carneiro, J., Saraiva, P., Martinho, D., Marreiros, G., \& Novais, P. (2018). Representing decision-makers using styles of behavior: An approach designed for group decision support systems. Cognitive Systems Research, (47), 109-132.

Carroll, J. M. (1997). Human-Computer Interaction: Psychology as a Science of Design. Annual Review of Psychology. 48, 61-83.

Carvalho, A. (2000). A representação do conhecimento segundo a Flexibilidade Cognitiva. Revista Portuguesa de Educação 13(1), 169-184.

Chaiklin, S. (2003). The zone of proximal development in Vygotsky's analysis of learning and instruction. In A. Kozulin, B. Gindis, V. Ageyev, \& S. Miller, Vygotsky's educational theory and practice in cultural context (pp. 39-64). Cambridge, England: Cambridge University Press.

Chapman, P. (1997). Models of engagement: Intrinsically motivated interaction with multimedia learning software. Waterloo, Canada: Unpublished master's thesis, University of Waterloo,.

Chibelushi, C., \& Bourel, F. (2003). Facial expression recognition: A brief tutorial overview. On-Line Compendium of Computer Vision. School of Computing, Staffordshire University.

Clark, R. (1983). The transfer of technology between nations. Interaction, 1(3), 185203.

Cohn, M., Mehl, M., \& Pennebaker, J. (2014). Markers of Linguistic Psychological Change Surrounding September 11, 2001. Psychological Science, 15(10), 687-693.

Conati, C. (2002). Probabilistic assessment of user's emotions in educational games. Applied Artificial Intelligence, 16(7-8), 555-575. 
Corchado, J., Bajo, J., Paz, J., \& Rodríguez, S. (2009). An execution time neuralCBR guidance assistant. Neurocomputing, 2743-2753.

Coutinho, C. (2005). Percursos da investigação em Tecnologia Educativa em Portugal: Uma abordagem temática e metodológica a publicações científicas (198-2000). Braga: Univerisidade do Minho.

D’Mello, S., \& Graesser, A. (2009). Automatic Detection of Learner'S Affect From Gross Body Language. Applied Artificial Intelligence.

Dai, W., Duch, W., Abdullah, A., Xu, D., \& Chen, Y.-S. (2015). Recent advances in learning theory. Computational intelligence and neuroscience, 14.

Dalgleish, T., \& Power, M. (1999). Handbook of cognition and emotion. Wiley Library.

Dalgleish, T., Dunn, B., \& Mobbs, D. (2009). Affective Neuroscience: Past, Present, and Future. Emotion Review, 1(4), 355-368.

Davenport, T., \& Beck, J. (2013). The attention economy: Understanding the new currency of business. Harvard Business Press.

De Lemos, J., Reza Sadeghnia, G., \& Ólafsdóttir, Í. (2008). Measuring emotions using eye tracking. Proceedings of Measuring Behavior, (p. 226).

Deater-Deckard, K., Chang, M., \& Evans, M. (2013). Engagement States and Learning from Educational Games. In F. Blumberg, \& S. Fisch, Digital Games: A Context for Cognitive Development. New Directions for Child and Adolescent Development (pp. (139), 21-30). Wiley online library.

Desimone, R., \& Duncan, J. (1995). Neural mechanisms of selective visual attention. Annual Review of Neuroscience, 18, 193-222.

Duc, A., Bays, P., \& Husain, M. (2008). Eye Movements as a Probe of Attention. In C. Kennard, \& J. Leigh, Progress in Brain Research (pp. 403-411). London: Elsevier. 
Duchowski, A. (2002). A breadth-first survey of eye-tracking applications. Behavior Research Methods, Instruments, \& Computers. A Journal of the Psychonomic Society, Inc, 34(4), 455-470.

Durães, D., Bajo, J., \& Novais, P. (2017). Analysis Learning Styles though Attentiveness. In P. Vittorini, R. Gennari, T. Di Mascio, S. Rodríguez, \& F. Prieta, Methodologies and Intelligent Systems for Technology Enhanced Learning. MIS4TEL 2017. Advances in Intelligent Systems and Computing (pp. (617)90-97). Springer, Cham.

Durães, D., Cardoso, C., Bajo, J., \& Novais, P. (2017). Learning Frequent Behaviors Patterns in Intelligent Environments for Attentiveness Level. In F. Prieta, Z. Vale, L. Antunes, T. Pinto, T. Campbell, J. Vicente , . . . M. Moreno, In International Conference on Practi-cal Applications of Agents and Multi-Agent Systems, PAAMS 2017: Trends in Cyber-Phys-ical Multi-Agent Systems. The PAAMS Collection - 15th International Conference, PAAMS 2017 (pp. 139147). Springer.

Durães, D., Carneiro, D., Bajo, J., \& Novais, P. (2016). Supervising and Improving Attentiveness in Human Computer Interaction. In P. Novais, \& S. Konomi, Intelligent Environments, vol.21 (pp. 255-264). IOS Press Ebooks.

Durães, D., Carneiro, D., Bajo, J., \& Novais, P. (2016). Using Computer Peripheral Devices to Measure Attentiveness. In F. Prieta, M. Escalona, R. Corchuelo, P. Mathieu, Z. Vale, A. Campbell, . . . M. Moreno, Trends in Practical Applications of Scalable Multi-Agent Systems, the PAAMS Collection (pp. 147-155). Switzerland : Springer Cham.

Durães, D., Carneiro, D., Jimenez, A., \& Novais, P. (2018). Characterizing Attentive Behavior in Intelligent Environments . Neurocomputing, 46-54.

Eggen, P., \& Kauchak, D. (1992). Educational psychology: classroom connections. New York: Merrill.

Ekman, P. (1992). An argument for basic emotions. Cognition \& emotion, 6(3-4), 169-200. 
Ekman, P., \& Friesen, W. (1969). The repertoire of nonverbal behavior: Categories, origins, usage, and coding. Semiotica, 1(1), 49-98.

Estes, W. (2014). Handbook of Learning and Cognitive Processes (Volume 4): Attention and Memory. Psychology Press.

Esysench, M. (1992). Anxiety: The cognitive Perspective. Hove, England: Erlbaum.

Eysenck, M., Derakshan, N., Santos, R., \& Calvo, M. (2007). Anxiety and cognitive performance: attentional control theory. American Psychological Association, Vol. 7, No.2, 336-353.

Faber, L., Maurits, N., \& Lorist, M. (2012). Menatl Fatigue Affects Visual Selective Attention. PLoS ONE 7(10), e48073.

Felder, R., \& Silverman, L. (2002). Learning and Teaching Styles in Engineering Education. Jornal of Engineering Education, 78(7), 674-681.

Folkard, S., \& Tucker, P. (2003). Shift Work, Safety and Productivity. Journal Carrees Network, Occupational Medicine, 95-101.

Ford, N., \& Chen, Y. (2000). Individual Differences, Hypermedia Navigation and Learning: an Empirical Study. Journal of Educational Multimedia and Hypermedia, 9(4), 281-311.

Fox, N. (1997). Dynamic cerebral processes underlying emotion regulation. In The development of emotion regulation: Biological and behavioral considerations. Monographs of the Society for Research in Child Development, 59, 152-166.

Fredricks, J., Blumenfeld, P., \& Paris, A. (2004). School engagement: Potential of the concept, state of the evidence. Review of Educational Research, 74(1), 59-109.

Gardell, B. (1982). Worker Participation and Autonomy: a Multilevel Approach to Democracy at the Workplace. International Journal of Health Services, volume $4,527-552$.

Gonçalves, S., Rodrigues, M., Carneiro, D., Fdez-Riverola, F., \& Novais, P. (2015). Boosting Learning: Ñon-instrutise Monitoring of Student's Efficiency. In T. Di 
Mascio, R. Gennari, P. Vittorini, \& F. Prieta, Methodologies and Intelligent Systems for Technology Enhanced Learning (pp. vol. 374, 73-80). Switzerland 2015: Springer, Cham.

Hall, M., Frank, E., Holmes, G., Pfahringer, B., Reutemann, P., \& Witten, I. (2009). The WEKA data mining software: an update. SIGKDD Explor. Newsl. 11, 1018.

Hamre, B., \& Pianta, R. (2006). Student-Teacher Relationships. Association of School Psychologists National .

Hassin, M., Aziz, A., \& Norwawi, N. (2004). Affective computing: knowing. The National Seminar of Science Technology and Social Science (STSS '04). UiTM Pahang.

Hernandez, J., Paredes, P., Roseway, A., \& Czerwinski, M. (2014). Under pressure: sensing stress of computer users. Proceedings of the SIGCHI conference on Human factors in computing systems. ACM, 51-60.

Hoffman, B., \& Nadelson, L. (2010). Motivational engagement and video gaming: A mixed methods study. Educational Technology Research \& Development, 58, 245-270.

Honey, P., \& Mumford, A. (2006). The Learning Styles Helper's Guide. London: Peters Honey Publications.

Hopkins, K. (1998). Educational and psychological measurement and evaluation (Vol. 8th ed.). Boston: Allyn and Bacon.

Huang, M., \& Alessi, N. (1999). Presence as an emotional experience. In: Westwood JD, Hoffman. In J. Westwood, H. Hoffman, R. Robb, \& D. Stredney, Medicine meets virtual reality: the convergence of physical and informational technologies options for a new era in healthcare (pp. 148-153). Amsterdam: IOS Press.

Hwang, K., \& Yang, C. (2009). Automated Inattention and Fatigue Detection System in Distance Education for Elementary School Students. Journal of Educational Technology 12, 22-35. 
Isen, A. (2001). An Influence of Positive Affect on Decision Making in Complex Situations: Theoretical Issues With Practical Implications. Journal of Consumer Psychology, 11(2), 75-85.

Itti, L., \& Koch, C. (2001). Computational modelling of visual attention. Nature Reviews Neuroscience volume , (2),194-203.

Jaimes, A., \& Sebe, N. (2007). Multimodal human-computer interaction: A survey. Computer Vision and Image Understanding, 108(1-2), 116-134.

James, W. (1890). Principles of Psychology (Vol. Vol.1). New York: Dover Publications Inc.

Jaques, P., \& Vicari, R.-M. (2005). Estado da arte em ambientes inteligentes de aprendizagem que consideram a afetividade do aluno. evista inform'atica na educac, ao: teoria \& prática, 8(1):15-38.

Joyce, E., Blumenthal, S., \& Wessely, S. (1996). Memory, attention, and executive function in chronic fatigue syndrome. Journal of neurology, neurosurgery, and psychiatry, 60(5), 495-503.

Kappelman, L. (1995). Measuring user involvement: Adiffusion of innovation perspective. Database Advances, 26(2/3), 65-86.

Keefe, J. (1979). Learning Style: An Overview. NASSP'S Student Learning Styles: Diagnosing and Prescribing Programs, 1-17.

Kejariwal, A., Kulkarni, S., \& Ramasamy, K. (2015). Real time analytics: algorithms and systems. Proceedings of the VLDB Endowment 8(12), 2040-2041.

Keltner, D., \& Ekman, P. (2000). Emotion: an overview. Encyclopedia of psychology, 3, 162-167.

Khurana, I. (2007). Textbook of Human Physiology for Dental Students. Elsevier Health Sciences APAC.

Kleinginna, P., \& Keinginna, A. (1981). A Categorized List of Emotion Definitions, with Suggestions for a Consensual Definition. Motivation and Emotions Vol. 5, N. ${ }^{\circ} 4$. 
koch, C., \& Ulman, S. (1987). Shifts in Selective Visual Attention: Towards the Underlying Neural Circuitry. In L. Vaina, Matters of Intelligence: Conceptual Structures in Cognitive Neuroscience (pp. (188) 115-141). Springer, Dordrecht.

Kolb, A., \& Kolb, D. (2005). Learning styles and learning spaces: enhancing experiential learning in higher education. Academy of Management Learning and Education, 4, 193-212.

Kolb, D. (1984). Experiential Learning: Experience as the Source of Learning and Development. New Jersey: Prentice-Hall Inc.

Kort, B., Reilly, R., \& Picard, R. (2001). An affective model of interplay between emotions and learning: Reengineering educational pedagogy-building a learning companion. Proceedings -IEEE International Conference on Advanced Learning Technologies, ICALT, (pp. 43-46).

Kuppermann, M., Lubeck, D., Mazonson, P., Patrick, D., Stewart, A., Buesching, D., \& Filer, S. (1995). Sleep problems and their correlates in a working population. Journal of General Internal Medicine 10(1), 25-32.

Lang, P., Bradley, M., \& Cuthbert, B. (1997). International Affective Picture System (IAPS): Technical Manual and Affective Ratings. Center for the Study of Emotion and Attention 1997. Psychology.

Lee, H., Choi, Y., Lee, S., \& Park, I. (2012). Towards Unobtrusive Emotion Recognition for Affective Social Communication. The 9th Annual IEEE Consumer Communications and Networking Conference - Special Session Affective Computing for Future Consumer Electronics. Las Vegas, NV, USA : IEEE.

Leventhal, H., \& Scherer, K. (1987). The relationship of emotion to cognition: A functional approach to a semantic controversy. Cognition and emotion, 1(1), 3-28.

Lorist, M., Klein, M., Nieuwenhuis, S., Jong, R., Mulder, G., \& Meijman, T. (2000). Mental fatigue and task control: planning and preparation. Psychophysiology, 37(5), 614-625. 
Macarro, A., Villafaina, E., Pinto, A., \& Bajo, J. (2011). Interaction Mechanism for Language Learning for Elderly People through Mobile Devices. In J. Bajo, J. Corchado, M. Moreno, V. Julián, P. Mathieu, J. Canada-Bago, . . . A. Fernández, Highlights in Practical Applications of Agents and Multiagent Systems. 9th International Conference on Practical Applications of Agents and Multiagent Systems (pp. 335-342). Springer Verlag.

MacQueen, J. (1967). Some methods for classification and analysis of multivariate observations. Proceedings of the 5th Berkeley Symposium on Mathematical Statistics and Probability, 1, 281-297.

Mancas, M. (2007). Computational Attention - Toward Attentive Computers. Press Universitaire de Louvain.

Mancas, M. (2015, October 6). Attention in computer science - Part 1. Retrieved December 31, 2016, from News and insights from EAl community - Blog: http://blog.eai.eu/attention-in-computer-science-part-1/

Mancas, M. (2015, November 26). Attention in computer science - Part 2. Retrieved December 31, 2016, from News and insights from EAl community - Blog: http://blog.eai.eu/attention-in-computer-science-part-2/

Mancas, M., Ferrera, V., Ritche, N., \& Taylor, J. (2016). Why Do Computers Need Attention? In M. Mancas, V. P. Ferrera, N. Ritche, \& J. G. Taylor, From Human Attention to Computational Attention: A Multidisciplinary Approach (pp. 1-6). New Yoork: Springer Science+Business Media LLC.

McDaniel, B., D’Mello, S., King, B., Chipman, P., Tapp, K., \& Graesser, A. (2007). Facial Features for Affective State Detection in Learning Environments.

McGaugh, J. (2004). The Amygdala Modulates the Consolidation of Memories of Emotionally arousing Experiences. Annu. Rev. Neurosci. 27, 1-28.

McLeod, S. (2008). Selective attention. Retrieved from Simply Psychology: https://www.simplypsychology.org/attention-models.html 
Meijman, T. (1997). Mental Fatigue and the Efficiency of Information Processing in Relation to Work Times. International Journal of Industrial Ergonomics 20(1), 31-38.

Milrad, M., Wong, L., Sharples, M., Hwang, G., Chee-Kit, L., \& Ogata, H. (2013). Seamless Learning: An International Perspective on Next Generation Technology Enhanced Learning. In Z. Berge, \& L. Muilenburg (Ed.). (pp. 95108). Routledge.

Mole, C. (2012). Attention. In E. N. Zalta, The Stanford Encyclopedia of Philosophy (p. https://plato.stanford.edu/archives/fall2013/entries/attention). Winter Edition.

Monrose, F., \& Rubin, A. (2000). Keystroke dynamics as a biometric for authentication. Future Generation Computer Systems, 16(4):351-359.

Morgan, R., Baker, F., \& Alabama, U. (2013). A Comparison of VARK Analysis Studies and Recommendations for Teacher Educators. Society for Information Technology \& Teacher Education International Conference (pp. 1381-1385). Formerly EdITLib-Education \& Information Technology Library.

Mota, S., \& Picard, R. (2003). Automated Posture Analysis for Detecting Learner's Interest Level. Conference on Computer Vision and Pattern Recognition Workshop, (pp. 5, 49).

Neto, W., Gauthier, F., \& Nassar, S. (2005). An Adaptive e-Learning Model for the Semantic Web. International Workshop on Applications of Semantic Web Technologies for E-Learning, (pp. 63-64). Banff, Canada.

Neu, D., Kajosch, H., Peigneux, P., Verbanck, P., Linkowski, P., \& Le Bon, O. (2010). Clinical complaints of daytime sleepiness and fatigue: How to distinguish and treat them, especially when they become'excessive'or'chronic'? Acta neurologica belgica, 110(1), 128-134.

Novais, P., \& Carneiro, D. (2016). The Role of Non-Intrusive Approaches in the Development of People-Aware Systems. PAI - Progress in Artificial Intelligence, vol. 5, issue 3, pp 215-220. 
O'Brien, H., \& Toms, E. (2008). What is user engagement? A conceptual framework for defining user engagement with technology. Journal of the Association for Information Science and Technology, Vol.59, Issue 6, 938-955.

Oitzl, M., \& Kloet, E. (1992). Selective corticosteroid antagonists modulate specific aspects of spatial orientation learning. Behav. Neurosci, 106, 62-71.

Olff, M., Langeland, W., \& Gersons, B. (2005). The Psychobiology of PTSD: coping with trauma. Psichoneuroendocrinology (30), 974-982.

Olofsson, J., Nordin, S., Sequeira, H., \& Polich, J. (2008). Affective picture processing: An integrative review of ERP findings. Biological Psychology, 77(3), 247-265.

Ortony, A. (2002). On making believable emotional agents believable. In R. Trappl, P. Petta, \& S. Payr, Emotions in humans and artifacts (p. 189). Cambridge: MIT Press.

Ortony, A., Clore, G., \& Collins, A. (1990). The cognitive structure of emotions. Cambridge Press.

Osgood, C., May, W., \& Miron, M. (1975). Cross cultural universals of affective meaning. University of Illinois Press.

Palmer, S., Cooper , C., \& Thomas, K. (2003). Creating a Balance: Managing Stress. London: British Library.

Pang, B., \& Lee, L. (2006). Opinion Mining and Sentiment Analysis. Foundations and Trends® in Information Retrieval, 1(2), 91-231.

Pantic, M., \& Rothkrantz, L. (2003). Toward an affect-sensitive multimodal humancomputer interaction. Proceedings of the IEEE 91, pp. (9)1370-1390.

Paradiso, S. (2002). Affective Neuroscience: The Foundations of Human and Animal Emotions. In American Journal of Psychiatry (Vol. 159). Oxford University Press. 
Partala, T., \& Surakka, V. (2003). Pupil size variation as an indication of affective processing. International Journal of Human Computer Studies, 59(1-2), 185198.

Pekrun, R. (2014). Emotions and Learning - educational and Pactices. International Academy of Education and International Bureau of Education, United Nations Eductional Scientific, 8-10.

Pennebaker, J., Booth, R., \& Francis, M. (2007). Linguistic Inquiry and Word Count Program: LIWC 2007. Word Journal Of The International Linguistic Association, 2-21.

Picard, R. (1997). Affective Computing. MIT Press.

Picard, R., Papert, S., Bender, W., \& Blumberg, B. (2004). Affective learning - a manifesto. BT Technology Journal 22(4), 253-268.

Pimenta, A., Carneiro, D., Neves, J., \& Novais, P. (2016). A Neural Network to Classify Fatigue from Human-Computer Interaction. Journal of Neurocomputing, 413-426.

Pimenta, A., Gonçalves, S., Carneiro, D., Fde-Riverola, F., Neves, J., \& Novais, P. (2015). Mental workload management as a tool in e-learning scenarios. 2015 International Conference on Pervasive and Embedded Computing and Communication Systems (PECCS) (pp. 25-32). IEEE.

Pimenta, A., Gonçalves, S., Carneiro, D., Riverola, F., \& Novais, P. (2015). Mental Workload Management as a Tool in e-Learning Scenarios. In B-Peces, O. Paillet, \& A. Ahrens, Proceedings of the 5th International Conference on Pervasive and Embedded Computing and Communication Systems (pp. 2532). Scitepress.

Posner, M., \& Rothbart, M. (2007). Educating the human brain. Washington, DC: American Psychological Association.

Pritchard, A. (2009). Ways of learnng - learning theories and learnig styles in the classroom. New York: Routldge.

Regan, K. (2003). EMOTION AND E-LEARNING. Darwin. 
Reschly, A., \& Christenson, S. (2012). Jingle, jangle, and conceptual haziness: Evolution and future directions of the engagement construct. In $A$. Christenson, A. Reschly, \& C. Wylie, Handbook of research on student engagement (pp. 3-19). Springer.

Rincon, J., Costa, A., Villarrubia, G., Julian, V., \& Carrascosa, C. (2018). Introducing dynamism in emotional agent societies. Neurocomputing, (272), 27-39.

Rodrigues, M. (2013). STUDENT MONITORING/COUNSELING IN E-LEARNING PLATFORMS - Thesis. Vigo, Sapin: University of Vigo.

Rodrigues, M., Gonçalves, S., Carneiro, D., Novais, P., \& Riverola, F. (2013). Keystrokes and Clicks: Measuring Stress on e-Learning Students. Management Intelligent System, vol. 220, 119-126.

Rodrigues, M., Novais, P., \& Santos, M. (2005). Future Challenges in Intelligent Tutoring Systems - A Famework, Recent Research Developments in Learning. In A. Méndez Villas, B. Gonzalez Pereira, J. Mesa González, \& J. Mesa González , Proceedings of the 3rd International Conference on multimedia and Information \& Communication Technologies in Education ( $m$ ICTE2005) (pp. 929-934). Publishers Formatex.

Rodrigues, M., Riverola, F., \& Novais, P. (2012). An approach to Assess Stress in Elearning Students. Proceedings of the 11th European Confernece on eLearning: ECEL, 461-468.

Roosendaal, B., \& MacGaugh, J. (1996). Amygdaloid nuclei lesions differentially affect glucorticoid-induced memory enhancement in a inhibitory avoidance. Task. Neurobiol, Learn. Mem, 65, 1-8.

Russell, J. (1980). A circumplex model of affect. Journal of Personality and Social Psychology, 39(6).

Said, N. (2004). An engaging multimedia design model. Proceedings of the 2004 Conference on Interaction Design and Children (pp. 169-172). New York: ACM. 
Sandi, C. (1997). Experience-dependence facilitating effect of corticosterone on spatial memory formation in the water maze. Eur. J. Neurosci, 9, 637-642.

Sandi, C., \& Rose, S. (1994). Corticosterone enhances log-term retention in oneday-old chicks trained in a weak passive avoidance learning paradig. Brain. Res, 647, 106-112.

Shaver, P., Schwartz, J., Kirson, J., \& O’Connor. (1987). Emotional knowledge: Further explorations of a prototype approach. Journal of Personality and Social Psychology, 52(6), 1061-1086.

Shors, T. (2006). Stressfull experience and Learning across Lifespan. Annu. Rev. Psychol, Home Annual Review of Psychology Volume 57, 55-85.

Sinatra, G., Heddy, B., \& Lombardi, D. (2015). The challenges of defining and measuring student engagement in science. Educational Psychologist, 50, 113.

Smith, L., \& Renzulli, J. (1984). Learning style preferences: A practical approach for classroom teachers. Theory Into Practice, 23(1), , 44-50.

Smith, P., \& Ragan, T. (1999). Instructional design. New York: Wiley.

Somekh, B. (2005). Action Research: A Methodology For Change And Development: A Methodology for Change and Development. New York: Open University Press.

Stricker, A. (2009). Why Affective Learning in a Situated Place Matters for the Millennial. Air University.

Tamiz, M., Karami, M., Mehorabi, I., \& Gidary, S. (2013). A Novel Attention Control Modeling Method for Sursor Selection Based on Fuzzy Neural Network Learning. First RSI/ISM International Conference on Robotics and Mechatronics (ICRoM).

Thamarasseri, I. (2017). MTEC202: Learners and the Learning Process - Unit IV Learning Environment and Assessment. KAshmir, India: Central University of Kashmir. 
Thompson, A., Simonson, M., \& Hargrave, C. (1996). Educational Technoogy: a review of the research. AECT Publications.

Toala, R., Gonçalves, F., Durães, D., \& Novais, P. (2018). Adaptive and Intelligent Mentoring to Increase User Attentiveness in Learning Activities, . in 16th Ibero-American Conference on Artificial Intelligence IBERAMIA 2018. Trujillo, Peru: Elsevier.

Treisman, A., \& Gelade, G. (1980). A feature-integration theory of attention. Cognitive psychology 12(1), 97-136.

van der Linden, D., \& Eling, P. (2006). Mental fatigue disturbs local processing more than global processing. Psychological research, 70(5), 395-402.

van der Linden, D., Frese, M., \& Meijman, T. (2003). Mental Fatigue and the Control of Cognitive Processes: Effects on Perseveration and Planning. Acta Psychologica 113(1), 45-65.

Watson, D., Wiese, D., Vaidya, J., \& Tellegen, A. (1999). The two general activation systems of affect: Structural findings, evolutionary considerations, and psychobiological evidence. Journal of personality and social psychology, 76(5):820.

Weber, W., Rabaey, J., \& Aarts, E. (1988). Ambient Intelligence. In R. Felder, \& L. Silverman, Learning and Teaching Styles in Engineering Education, Engineering Education, 78(7) (pp. 674-681).

Wu, S., Falk, T., \& Chan, W.-Y. (2011). Automatic speech emotion recognition using modulation spectral features. Speech Communication, 53(5), 768-785.

Xu, Z. (2001). Problems and strategies of teaching English in large classes in the People's Republic of China. In A. Herrmann, \& M. Kulski , Expanding Horizons in Teaching and Learning. Proceedings of the 10th Annual Teaching Learning Forum. Perth: Curtin University of Technology.

Yampolskiy, R., \& Govindaraju, V. (2008). Behavioural Biometrics: a Survey and Classification. International Journal of Biometrics, 81-113. 
Yu-Fei, M., \& Hong, J. (2003). Contrast-based Image Attention Analysis by Using Fuzzy Growing. ACM Multimedia, 374-381.

Zhou, X., Dai, G., Huang, S., Sun, X., Hu, F., Hu, H., \& Ivanovic', M. (2015). Cyberpsychological computation on social community of ubiquitous learning. Computational intelligence and neuroscience, 12.

Zimmermann, P., Guttormsen, S., Danuser, B., \& Gomez, P. (2003). Affective computing - a rationale for measuring mood with mouse and keyboard. International journal of occupational safety and ergonomics, 9(4):539-551. 